This publication may be purchased from the Superintendent of Documents. U S Government Printing Office. Purchasing information for this or other Energy Information Administration (E|A) publications may be obtained from the Government Printing Office or ElA's National Energy Information Cenier. Questions on energy statistics should be directed to the Center by mail. telephone. or telecommunications device for the hearing imparred. Addresses. telephone numbers. and hours are as follows

\author{
National Energy Information Center. El-231 \\ Energy Information Administration \\ Forrestal Bullding. Room iF-048 \\ Washington. DC 20585 \\ (202) $586 \cdot 8800$ \\ Telecommunications Device for the \\ Hearing Impaired Only: (202) 586-1181 \\ 8 a.m. - 5 p.m. eastern time. M-F
}

Superintendent of Documents U.S. Government Printing Office Washington, DC 20402

(202) 783.3238

FAX 1-202-512-2233

8 a.m. -5 p.m., eastern time, M-F

We thank the following for the use of their photographs in this report:

Consolidation Coal Company: cover

Vorfolk Southern Corporation: page 9

TECO Transportation \& Trade: page 23

Central Louisiana Electric Company, Inc: page 41

Thunder Basin Coal Company: page 103

BHP Lah Minerals: page 10 ?

\title{
Cover Photo
}

Barges transport coal on the Monongahela River to a Pittsburgh power plant.

Released for Printing: October 21, 1991

The Coal Distribution (ISSN 0737-4399) is published quarterly by the Energy Information Administration. 1000 Independence Avenue. SW. Washington. DC 20585. and sells for $\$ 21.00$ per year (price subject to change without advance notice) Second-class postage pard at Washington DC 20066-9998. and additional malling offices POSTMASTER Send address changes to Coal Distribution. Energy information Administra tion. El-231. 1000 Independence Avenue. SW. Washington. DC 20585 


\section{Coal Distribution January-June 1991}

\section{Energy Information Administration}

Office of Coal, Nuclear, Electric and Alternate Fuels

U.S. Department of Energy Washington DC 20585

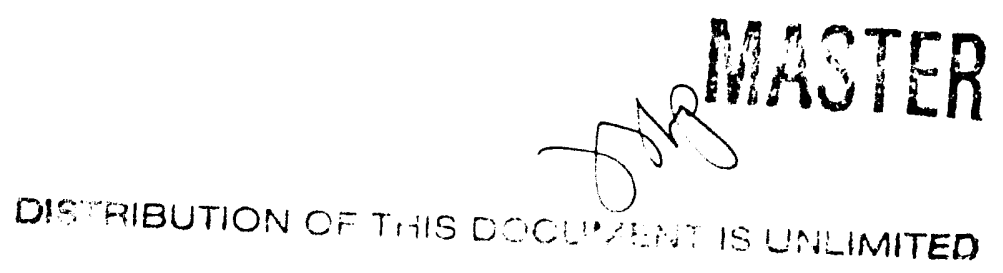




\section{Contacts}

General information for this document may be obtained from Robert Schnapp (202-254-5392), Director of the Coal Division, Office of Coal, Nuclear, Electric and Alternate Fuels, Energy Information Administration, or Noel C. Balthasar (202-254-5405), Chief of the Coal Data Systems Branch.
Specific information about the data in this report may be obtained from Bruce Quade (202-254-5461), Stephen Scott (202-254-5467), or Rebecca McNerney (202-254-5469). 


\section{Preface}

The Coal Distribution report provides information on coal production, distribution, and stocks in the United States to a wide audience including Congress. Federal and State agencies, the coal industry, and the general public. The data in this report are collected and published by the Energy Information Administration (EIA) to fulfill its data collection and dissemination responsibilities as specified in the Federal Energy Administration Act of 1974 (Public Law 93-275. Sections 5 and 13 , as amended).

This issue presents information for January through June 1991. Coal distribution data are shown (in Tables 1-34) by coal-producing State of origin. consumer use. method of transportation, and State of destination. All data in this report were collected by the EIA on Form EIA-6, "Coal Distribution Report." A copy of the form and the instructions for filing appear in Appendix B.

All data in this report for 1991 are preliminary. Data for previous years are final.
Beginning in 1989. EIA initiated a reporting change for the Form EIA-6, "Coal Distribution Report." This change, the collection of both coal-producing District and State of origin data. Was the first step in a program to eventually transition to a State-only basis and eliminate reporting by District.

Beginning with the first quarter of 1990, data are collected and published by State of origin only.

The Coal Distribution report continues the quarterly series started in 1957 by the Bureau of Mines. Department of the Interior, as a Mineral Industry Survey, Distribution of Bituminous Coal and Lignite Shipments. The last Bureau of Mines publication in this continuing series covered data for January through March 1977. Since that time the EIA has published these reports.

The Office of Coal, Nuclear. Electric and Alternate Fuels gratefully acknowledges the cooperation of the respondents in supplying the detailed data that make this report possible. 


\section{Contents}

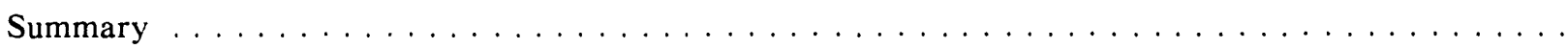

Consuming Sectors

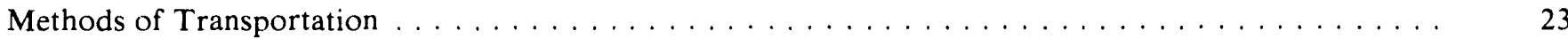

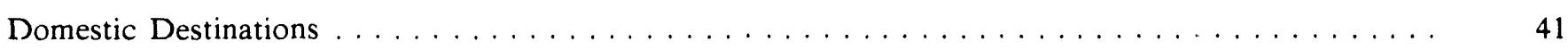

Appendices

A. Explanatory Notes $\ldots \ldots \ldots \ldots \ldots \ldots \ldots \ldots \ldots \ldots \ldots \ldots \ldots \ldots \ldots \ldots$

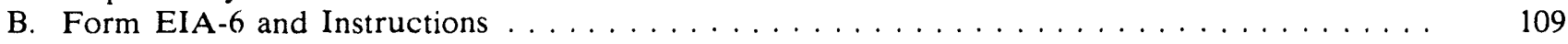

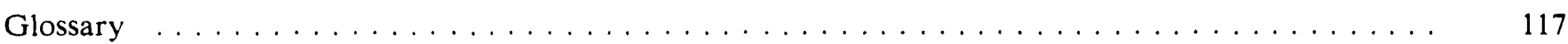




\section{Illustrations}

Page

1. Quarterly Coal Production and Purchases. Distribution. and Producer/Distributor Stocks,

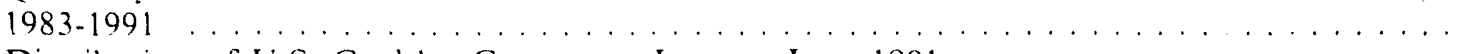

2. Distribution of L.S. Coal by Consurner, January-June $1991 \ldots \ldots \ldots$ 2

3. Quarterly Domestic Coal Distribution By Consumer Category, 1983-1991 . . . . . . . . . . . 13

4. Domestic Distribution of L.S. Coal by Method of Transportation, January-June 1991 . . . . 24

5. Quarterly Domestic Coal Distribution by Method of Transportation, 1983-1991 . . . . . . . . 30

6. Domestic Distribution of L.S. Coal by Leading State Destination, January-June $1991 \ldots \ldots$ 
U.S. Coal Production and Purchases, Distribution, and Stocks by Origin $\ldots \ldots \ldots \ldots \ldots$

U.S. Coal Production and Purchases by Origin ....................

U.S. Producers' and Distributors' Coal Stocks by Origin $\ldots \ldots \ldots \ldots \ldots \ldots \ldots \ldots$

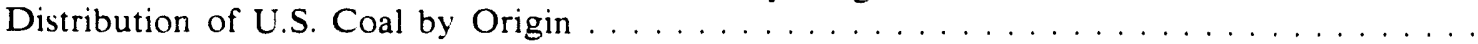

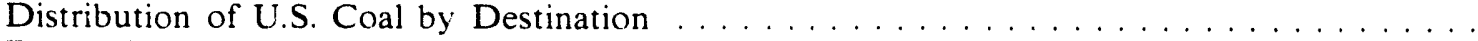

Domestic Distribution of U.S. Coal by Origin and Consumer, January-June 1991 . . . . . .

Foreign Distribution of U.S. Coal by Origin and Consumer, January-June $1991 \ldots \ldots$. . . . .

Domestic Distribution of U.S. Coal by Destination and Consumer, January-June $1991 \ldots \ldots$

Domestic Distribution of U.S. Coal to Electric Utility Plants by Origin . . . . . . . . . .

Domestic Distribution of U.S. Coal to Electric Utility Plants by Destination . . . . . . . . .

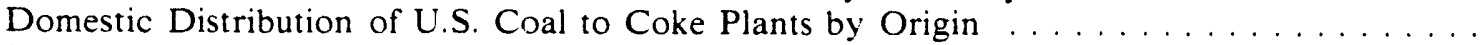

Domestic Distribution of U.S. Coal to Coke Plants by Destination . . . . . . . . . . .

Domestic Distribution of U.S. Coal to Industrial Plants (Except Coke) by Origin . . . . . . .

Domestic Distribution of U.S. Coal to Industrial Plants (Except Coke) by Destination . . . . .

Domestic Distribution of U.S. Coal to the Residential and Commercial Sector by Origin ... Domestic Distribution of U.S. Coal to the Residential and Commercial Sector by Destina-

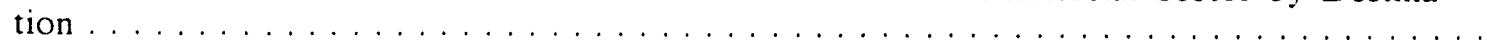

17. Domestic Distribution of U.S. Coal by Origin and Method of Transportation, January-June 1991

18. Foreign Distribution of U.S. Coal by Origin and Method of Transportation, January-June

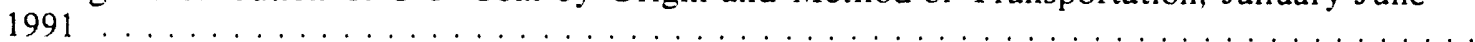

19. Domestic Distribution of U.S. Coal by Destination and Method of Transportation, JanuaryJune 1991

Secondary Methods of Transportation by Origin, January-June $1991 \ldots \ldots \ldots \ldots \ldots \ldots \ldots$

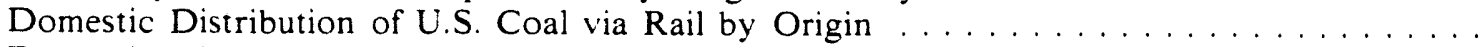

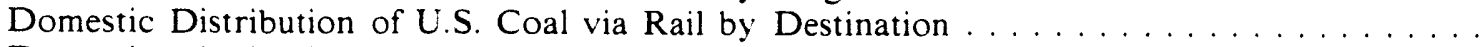

Domestic Distribution of U.S. Coal via River by Origin $\ldots \ldots \ldots \ldots \ldots \ldots \ldots \ldots$

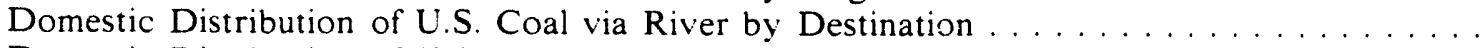

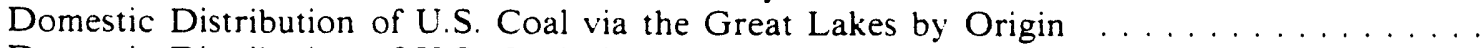

Domestic Distribution of U.S. Coal via the Great Lakes by Destination . . . . . . . . .

Domestic Distribution of U.S. Coal via Tidewater by Origin $\ldots \ldots \ldots \ldots \ldots \ldots \ldots$

Domestic Distribution of U.S. Coal via Tidewater by Destination $\ldots \ldots \ldots \ldots \ldots \ldots \ldots$

Domestic Distribution of U.S. Coal via Truck by Origin $\ldots \ldots \ldots \ldots \ldots \ldots \ldots$

Domestic Distribution of U.S. Coal via Truck by Destination $\ldots \ldots \ldots \ldots \ldots \ldots \ldots \ldots$

Domestic Distribution of U.S. Coal via Tramway, Conveyor, and Slurry Pipeline by Origin

Domestic Distribution of U.S. Coal via Tramway, Conveyor, and Slurry Pipeline by Desti-

nation

33. Domestic Distribution of U.S. Coal by Origin State. Consumer, Destination and Method of Transportation, January-June 1991

Domestic Distribution of U.S. Coal by Destination State, Consumer, Origin and Method of Transportation, January-June 1991 


\section{Summary}

U.S. coal production' from April through June 1991 was 237 million short tons, the lowest amount for any quarter since the second quarter of 1988 (Table 1). This brought the total for the first half of 1991 to 491 million short tons, which was 27 million short tons less than a year earlier (Table 2). Compared with the first 6 months of 1990, coal production for January through June declined in every State except for Wyoming and Arizona. Wyoming was the top coal-producing State, followed by West Virginia and Kentucky. The largest decreases in coal production occurred in Kentucky, Pennsylvania, and Indiana. Along with the decline in coal production, there was an equivalent reduction in coal distribution (Table 4), since the increase in producers' and distributors' coal stocks was about the same as in the comparable period last year.

The principal reason for the relatively low coal output in the first half of 1991 was a slowdown in the rate of coal stock replenishment by electric utilities. A total of 6 million short tons was added to utility stocks in the first 6 months of 1991. By contrast, in the same period in 1990, electric utilities built up their stockpiles by 25 million short tons. That build-up was in response to coal stocks ending 1989 at their lowest level since 1978.

Coal consumption at electric utilities from January through June 1991 was 4 million short tons more than a year earlier, as electricity demand went up slightly. Generation from nuclear, coal, and gas-fired units was used to offset decreased petroleum-fired and hydroelectric generation.

Kentucky coal production in the first 6 months of 1991 fell by 11 million short tons, primarily due to reduced demand from electric utiiities in the State as well as in Ohio and Florida. In Kentucky, electric utilities drew down coal stocks to meet demand for coal-fired generation, whereas in the first half of 1990, they built up coal stocks. In Ohio and Florida, the rate of coal stock replenishment at electric utilities was lower than in the same period last year. Coal production in Pennsylvania declined 5 million short tons, primarily due to a drop in the demand for coal by electric utilities in the State. Although demand for electricity in the State was down. coal-fired generation went up, replacing some nuclear and hydroelectric generation. However, electric utilities met the demand for coal by drawing from coal stocks. In contrast, a year earlier, the utilities were adding to coal stocks. To a lesser extent, coal production in Pennsylvania went down due to lower demand for coal from foreign markets and coke plants in the State. Coal production in Indiana dropped 3 million short tons, primarily due to withdrawals from coal stocks at electric utilities and reduced demand for electricity generation in the State. In comparison, in the first 6 months of 1990, Indiana's electric utilities raised the level of coal stocks.

Coal production in Wyoming was up 8 million short tons over the same period in 1990, primarily due to an increase in coal demand from electric utilities in Texas, Arkansas, and Missouri and, to a lesser extent, to higher coal shipments to overseas markets. In Texas, Arkansas, and Missouri, electric utilities relied on coal- fired generation to meet an upswing in electricity demand. In addition, electric utilities in Texas built up coal stocks, while in the first half of 1990 they dri:w down coal stocks. In Arkansas, the rate of coal stock replenishment exceeded the rate of increase in the first 6 months of 1990. Coal production in Arizona was up 1 million short tons due to the use of coal-fired generation to meet higher electricity demand in Nevada and a build-up of coal stocks at electric utilities in both Arizona and Nevada.

Coal distribution to coke plants in the first 6 months of 1991 fell 32 percent, compared with the first half of 1990 (Table 12). This drop was due to a downturn in the demand for coke, reflecting the slump in domestic steel production. Coke plants in Pennsylvania accounted for the largest decrease in coal demand in this sector.

Coal destined for Canada dropped 31 percent compared with January through June 1990, due to lower cemand for shipments to coke plants and electric utility markets in that country (Table 7). However, coal for overseas markets remained virtually the same.

Sources: Energy Information Administration, Electric Power Monthly (March 1991); Electric Power Monthly (September 1991); Coal Distribution (October 1990).

Includes purchases 
Figure 1. Quarterly Coal Production and Purchases, Distribution, and Producer/ Distributor Stocks, 1983-1991

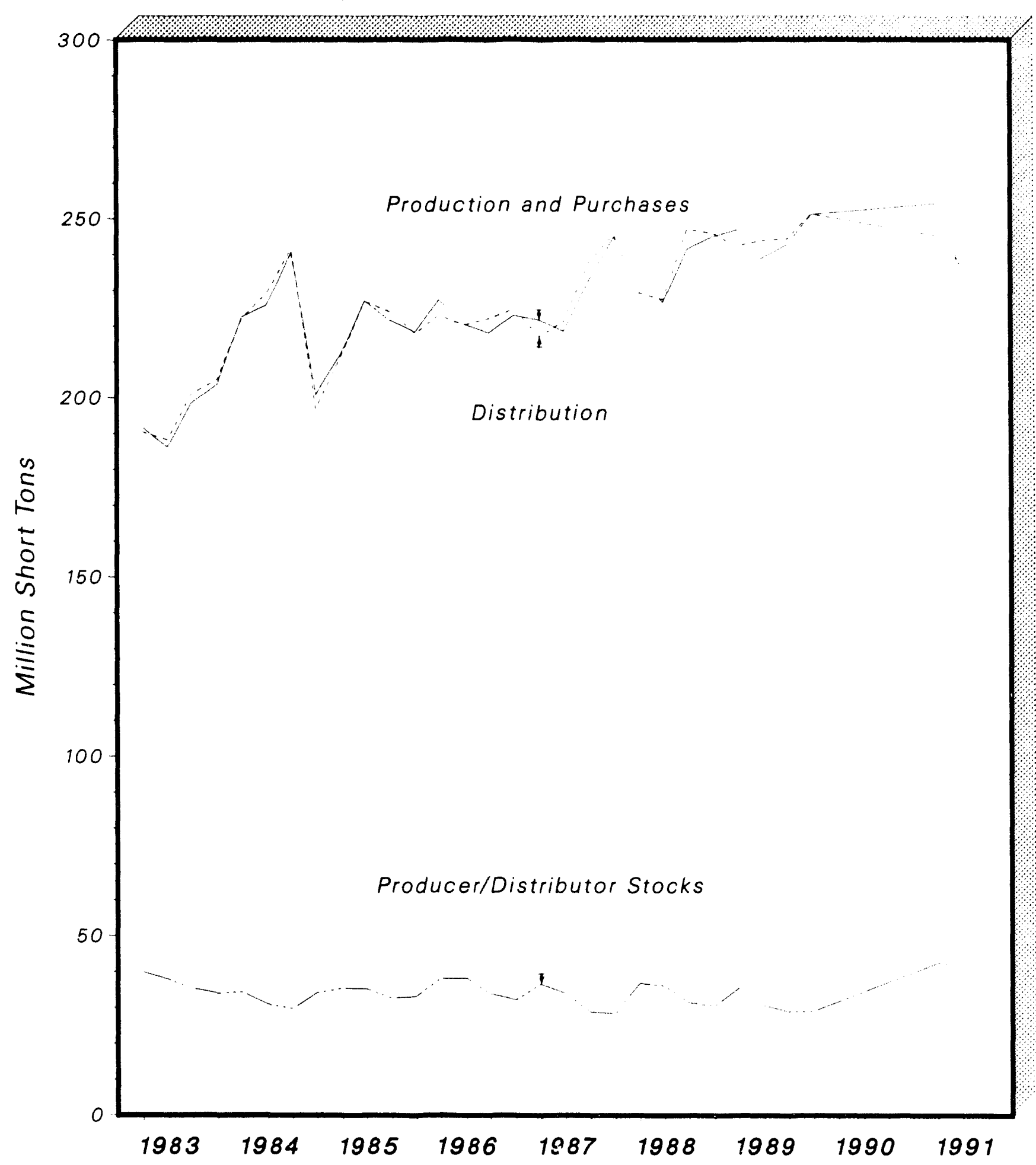

Note: Stocks are reported as of the end of each quarter. Each increment represents end of quarter data.

Source: Energy Information Administration. Form ElA-6, "Coal Distribution Report." 
Table 1. U.S. Coal Production and Purchases, Distribution, and Stocks by Origin (Thousand Short Tons)

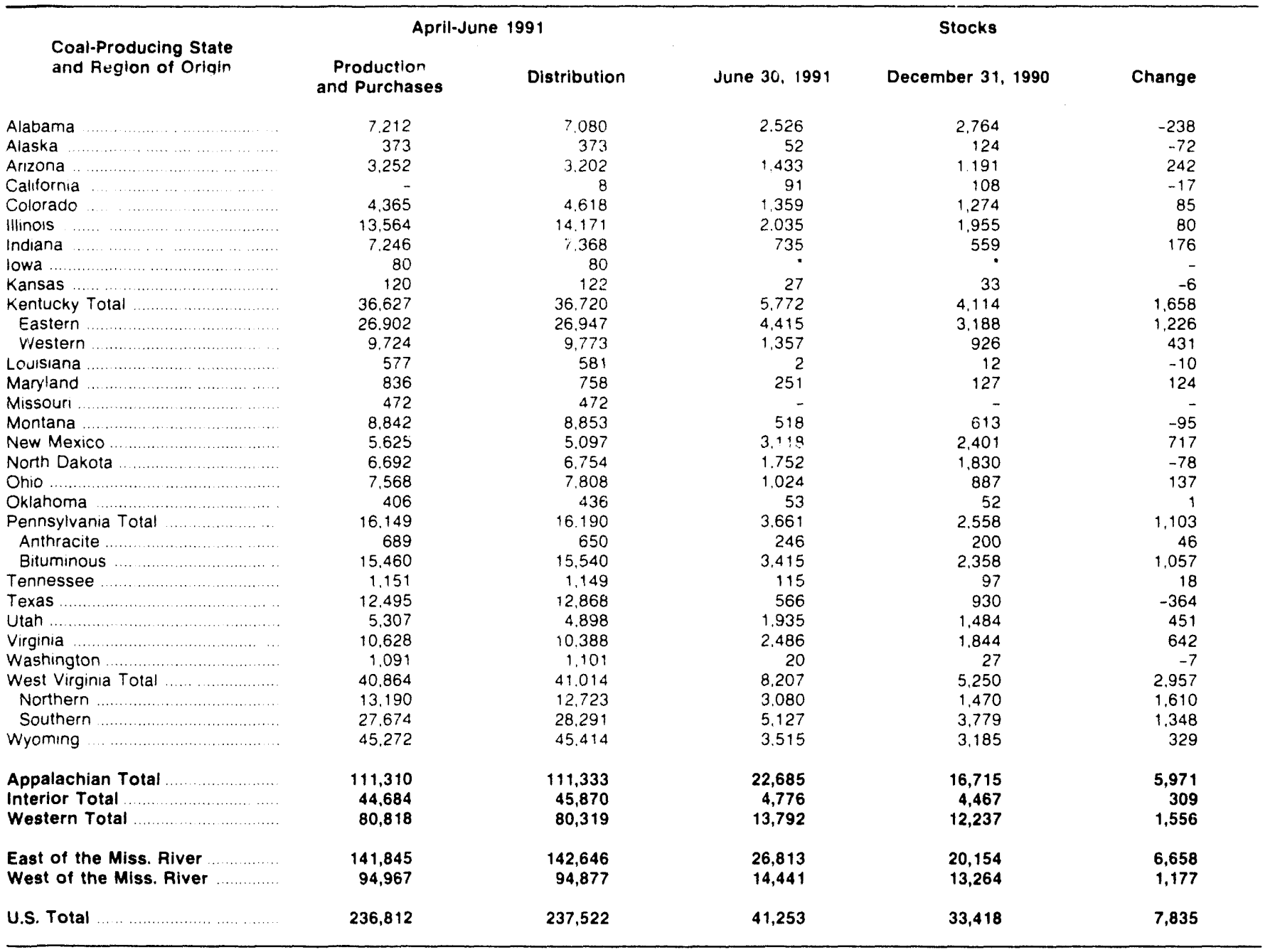

- Quantity is less than 500 short tons or percent is less than 05

Note: Total may not equal sum of components because of independent rounding

Source: Energy Information Administration. Form ElA.6. "Coal Distribution Report." 
Table 2. U.S. Coal Production and Purchases by Origin

(Thousand Short Tons)

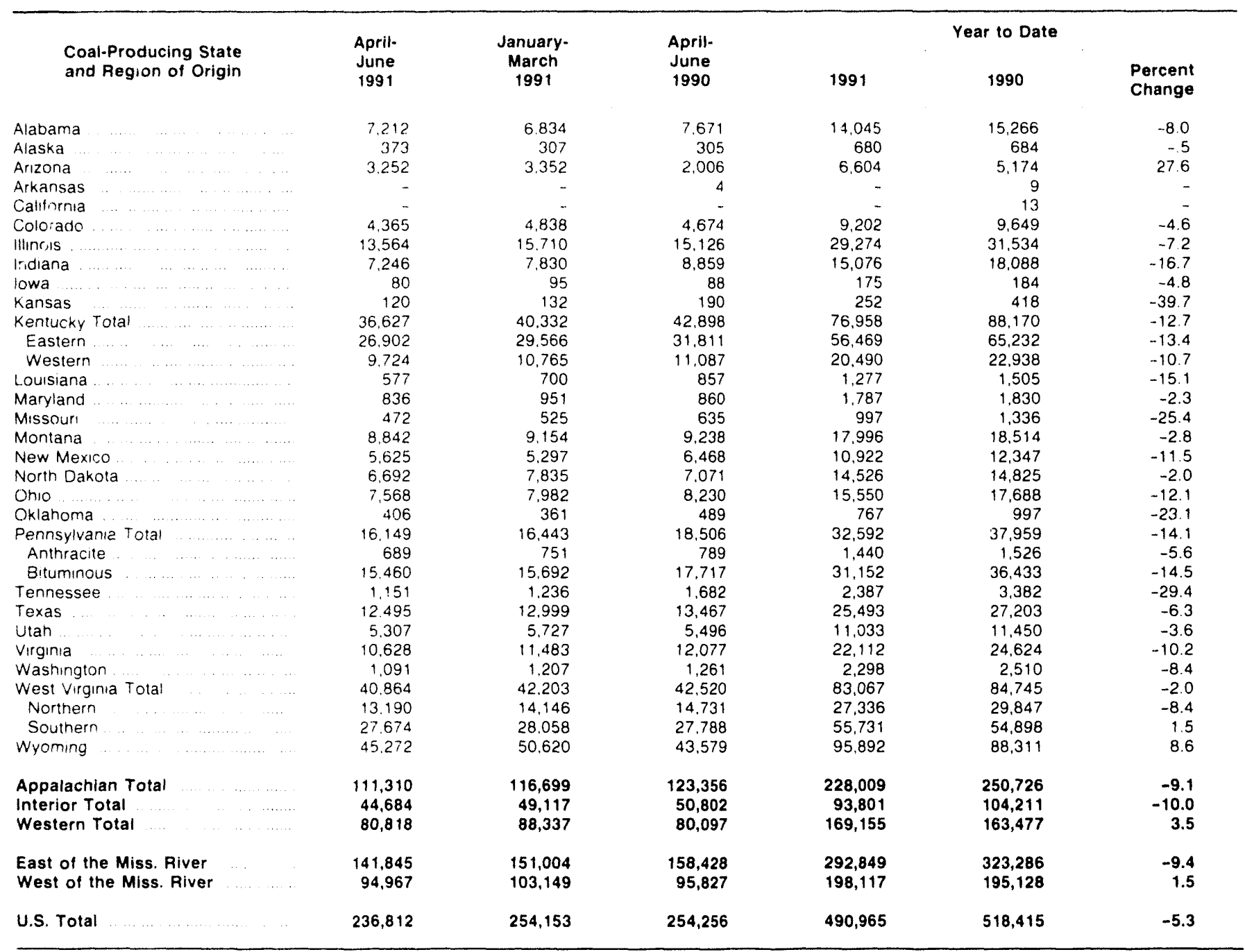

Note. Total may not equal sum of comporients because of independent rounding

Source Energy Information Administration. Form ElA.6. "Coal Distribution Report. 
Table 3. U.S. Producers' and Distributors' Coal Stocks by Origin (Thousand Short Tons)

\begin{tabular}{|c|c|c|c|c|}
\hline $\begin{array}{l}\text { Coal-Producing State } \\
\text { and Region of Origin }\end{array}$ & June 30,1991 & March 31, 1991 & June 30,1990 & $\begin{array}{c}\text { Percent Change } \\
\text { June } 30 \\
1991 \text { versus } 1990\end{array}$ \\
\hline Alabama & 2.526 & 2.400 & 2,808 & -10.0 \\
\hline Alaska & 52 & 52 & 124 & -58.1 \\
\hline 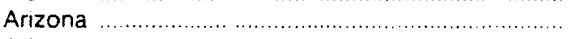 & 1,433 & 1,383 & 1,369 & 4.6 \\
\hline Arkansas & - & - & 2 & - \\
\hline 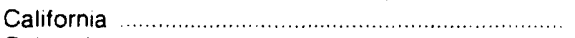 & 91 & - & 73 & 24.8 \\
\hline Colorado & 1.359 & 1.622 & 1.183 & 14.9 \\
\hline Illinois & 2.035 & 3,243 & 2.289 & -11.1 \\
\hline Indiana & 735 & 867 & 592 & 24.1 \\
\hline 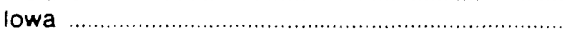 & 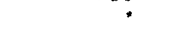 & . & . & NM \\
\hline 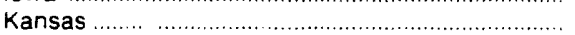 & 27 & 29 & 14 & 86.6 \\
\hline 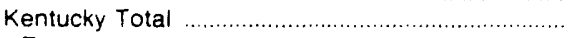 & 5.772 & 5,674 & 5.646 & 2.2 \\
\hline Eastern & 4,415 & 4,276 & 4378 & .8 \\
\hline 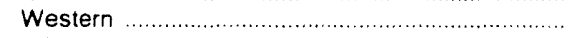 & 1,357 & 1,398 & 1,268 & 7.1 \\
\hline 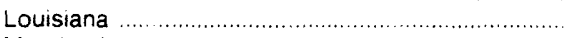 & 2 & 6 & 12 & -82.0 \\
\hline 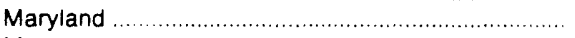 & 251 & 173 & 217 & 15.8 \\
\hline 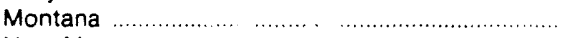 & 518 & 555 & 758 & -31.7 \\
\hline 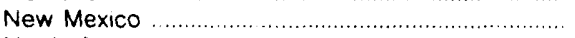 & 3,118 & 2,608 & 2,526 & 23.5 \\
\hline 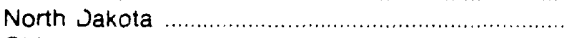 & 1,752 & 1,815 & 1,962 & -10.7 \\
\hline Ohio & 1,024 & 1.273 & 688 & 48.9 \\
\hline 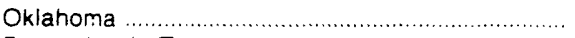 & 53 & 56 & 56 & -5.3 \\
\hline 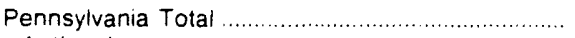 & 3.661 & 3.699 & 2.859 & 28.0 \\
\hline Anthracite & 246 & 211 & 324 & -24.3 \\
\hline 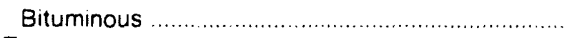 & 3,415 & 3,488 & 2.534 & 34.7 \\
\hline 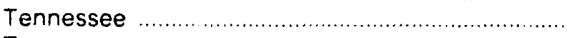 & 115 & 93 & 119 & -3.0 \\
\hline Texas & 566 & 939 & 894 & -36.7 \\
\hline Utah & 1,935 & 1,528 & 1,848 & 4.7 \\
\hline 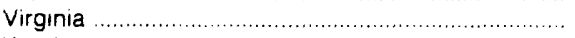 & 2,486 & 2,165 & 2.052 & 21.2 \\
\hline 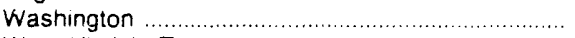 & 20 & 30 & 65 & -69.7 \\
\hline 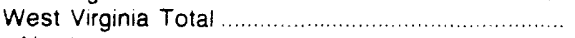 & 8,207 & 8,574 & 5.575 & 47.2 \\
\hline 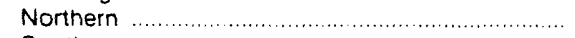 & 3,080 & 2,691 & 1.764 & 746 \\
\hline Southern & 5,127 & 5,883 & 3,811 & 34.5 \\
\hline 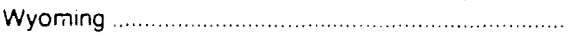 & 3,515 & 3.664 & 3.164 & 11.1 \\
\hline 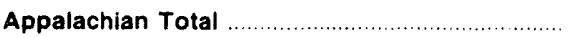 & 22,685 & 22,652 & 18,695 & 21.3 \\
\hline Interior Total & 4,776 & 6,537 & 5,127 & -6.9 \\
\hline Western Total & 13,792 & 13,257 & 13,073 & 5.5 \\
\hline East of the Miss. River & 26,813 & 28,159 & 22,845 & 17.4 \\
\hline 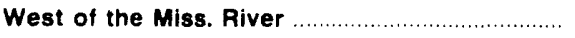 & 14,441 & 14,287 & 14,050 & 2.8 \\
\hline (2) & 41,253 & 42,446 & 36,895 & 11.8 \\
\hline
\end{tabular}

- Quantity is less than 500 short tons or percent is less than .05.

NM Not meaningful as value is greater than or equal to 500 .

Note: Total may not equal sum, of components because of independent rounding.

Source: Energy information Administration, Form EIA-6, "Coal Distribution Report." 
Table 4. Distribution of U.S. Coal by Origin (Thousand Short Tons)

\begin{tabular}{|c|c|c|c|c|c|c|}
\hline $\begin{array}{l}\text { Coal-Producing State } \\
\text { and Region of Origin }\end{array}$ & $\begin{array}{l}\text { April- } \\
\text { June } \\
1991\end{array}$ & $\begin{array}{c}\text { January- } \\
\text { March } \\
1991\end{array}$ & $\begin{array}{c}\text { April- } \\
\text { June } \\
1990\end{array}$ & 1991 & ar to Dat & $\begin{array}{l}\text { Percent } \\
\text { Change }\end{array}$ \\
\hline Alabama & 7,080 & 7.161 & 7.613 & 14,241 & 15,298 & -6.9 \\
\hline 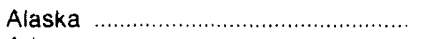 & 373 & 379 & 307 & 752 & 688 & 9.3 \\
\hline 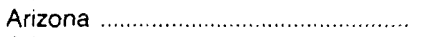 & 3,202 & 3,160 & 2,106 & 6,362 & 5,138 & 23.8 \\
\hline 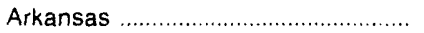 & - & - & 3 & - & 7 & - \\
\hline 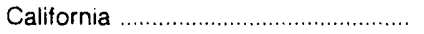 & 8 & - & 11 & 8 & 44 & -81.7 \\
\hline 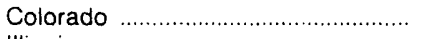 & 4,618 & 4.482 & 4,606 & 9,100 & 9,291 & -2.1 \\
\hline Illinois & 14,171 & 14,422 & 15,527 & 28,593 & 31,627 & -9.6 \\
\hline 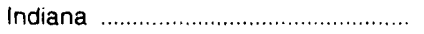 & 7,368 & 7,512 & 8,937 & 14,880 & 17,889 & -16.8 \\
\hline 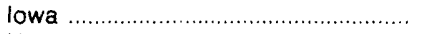 & 80 & 95 & 88 & 175 & 184 & -4.8 \\
\hline 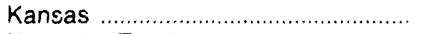 & 122 & 135 & 187 & 258 & 419 & -38.5 \\
\hline 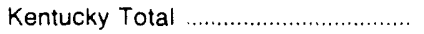 & 36.720 & 39,154 & 42,882 & 75,873 & 86,256 & -12.0 \\
\hline 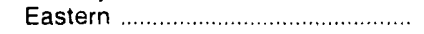 & 26,947 & 28,800 & 31,692 & 55,746 & 63,539 & -12.3 \\
\hline 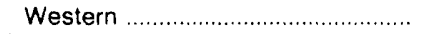 & 9.773 & 10,354 & 11,190 & 20,127 & 22.717 & -11.4 \\
\hline 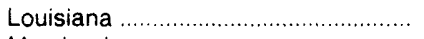 & 581 & 707 & 854 & 1,287 & 1,502 & -14.3 \\
\hline 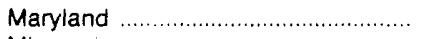 & 758 & 925 & 805 & 1,684 & 1.789 & -5.9 \\
\hline 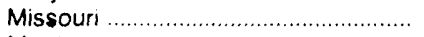 & 472 & 525 & 635 & 997 & 1.336 & -25.4 \\
\hline 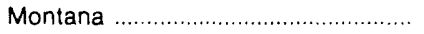 & 8,853 & 9,197 & 9,050 & 18,050 & 18,385 & -1.8 \\
\hline 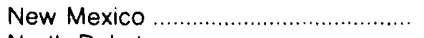 & 5,097 & 5,090 & 6.327 & 10,188 & 11,940 & -14.7 \\
\hline 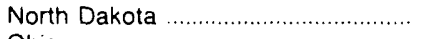 & 6,754 & 7,850 & 6,980 & 14,604 & 14,726 & -.8 \\
\hline Ohio & 7,808 & 7,593 & 8,133 & 15,400 & 17,352 & -11.3 \\
\hline 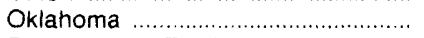 & 436 & 357 & 473 & 793 & 965 & -17.8 \\
\hline Pennsylvania Total ............................... & 16,190 & 15,242 & 18,332 & 31,432 & 37,021 & -15.1 \\
\hline 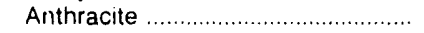 & 650 & 722 & 716 & 1.372 & 1,426 & -3.8 \\
\hline 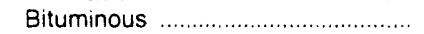 & 15,540 & 14,520 & 17,616 & 30,059 & 35,595 & -15.6 \\
\hline Tennessee & 1,149 & 1,246 & 1,709 & 2,394 & 3,376 & -29.1 \\
\hline 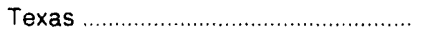 & 12,868 & 12,990 & 13,369 & 25,858 & 26,819 & -3.6 \\
\hline 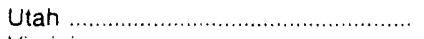 & 4,898 & 5,636 & 4.882 & 10,534 & 10.477 & 6 \\
\hline 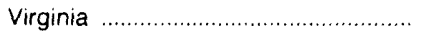 & 10,388 & 11,148 & 12,011 & 21,537 & 23,897 & -9.9 \\
\hline Washington & 1,101 & 1,204 & 1.221 & 2,305 & 2,471 & -6.7 \\
\hline 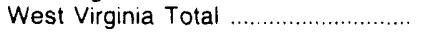 & 41,014 & 38,830 & 42,765 & 79,843 & 83,032 & -3.8 \\
\hline 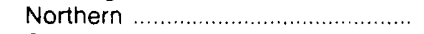 & 12,723 & 12,913 & 14,676 & 25,636 & 29,443 & -12.9 \\
\hline 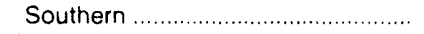 & 28,291 & 25,917 & 28,089 & 54,208 & 53.589 & 1.2 \\
\hline 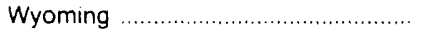 & 45,414 & 50,121 & 43,360 & 95,534 & 88,033 & 8.5 \\
\hline 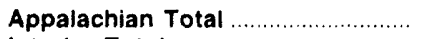 & 111,333 & 110,944 & 123,061 & 222,277 & 245,305 & -9.4 \\
\hline 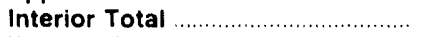 & 45,870 & 47,097 & 51,262 & 92,967 & 103,464 & -10.1 \\
\hline 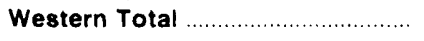 & 80,319 & 87,119 & 78,849 & 167,439 & 161,193 & 3.9 \\
\hline $\begin{array}{l}\text { East of the Miss. River } \\
\text { West of the Miss. River } \ldots \ldots \ldots \ldots \ldots \ldots \ldots \ldots\end{array}$ & $\begin{array}{r}142,646 \\
94,877\end{array}$ & $\begin{array}{l}143,232 \\
101,929\end{array}$ & $\begin{array}{r}158,715 \\
94,457\end{array}$ & $\begin{array}{l}285,877 \\
196,806\end{array}$ & $\begin{array}{l}317,538 \\
192,424\end{array}$ & $\begin{array}{r}-10.0 \\
2.3\end{array}$ \\
\hline 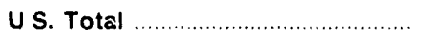 & 237,522 & 245,161 & 253,172 & 482,683 & 509,962 & -5.3 \\
\hline
\end{tabular}

Note: Total may not equal sum of components because of independent rounding.

Source: Energy Information Administration, Form ElA-6. "Coal Distribution Report." 
Table 5. Distribution of U.S. Coal by Destination (Thousand Short Tons)

\begin{tabular}{|c|c|c|c|c|c|c|}
\hline $\begin{array}{l}\text { Census Division and } \\
\text { State of Destination }\end{array}$ & $\begin{array}{l}\text { April- } \\
\text { June } \\
1991\end{array}$ & $\begin{array}{c}\text { January- } \\
\text { March } \\
1991\end{array}$ & $\begin{array}{l}\text { April- } \\
\text { June } \\
1990\end{array}$ & 1991 & ear to Dat & $\begin{array}{l}\text { Percent } \\
\text { Change }\end{array}$ \\
\hline New England Tutal. & 1,280 & 1,550 & 1,417 & 2,830 & 2,995 & -5.5 \\
\hline Connecticut ................. & 241 & 286 & 437 & 527 & 684 & -23.1 \\
\hline 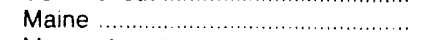 & 11 & 1 & 16 & 12 & 60 & -79.2 \\
\hline Massachusetts ......... & 681 & 890 & 821 & 1,572 & 1.803 & -12.8 \\
\hline 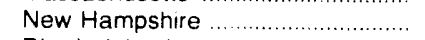 & 339 & 371 & 141 & 710 & 444 & 59.9 \\
\hline 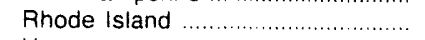 & $\bullet$ & 1 & $*$ & 1 & 1 & -46.5 \\
\hline 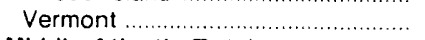 & 8 & 1 & 1 & 9 & 3 & 255.7 \\
\hline 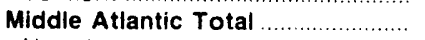 & 16,930 & 17,042 & 19,483 & 33,973 & 40,521 & -16.2 \\
\hline 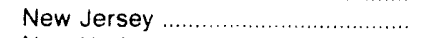 & 399 & 576 & 527 & 975 & 1,192 & -18.2 \\
\hline 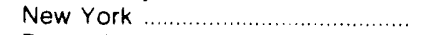 & 3,273 & 3,219 & 3,651 & 6.492 & 7,508 & -13.5 \\
\hline 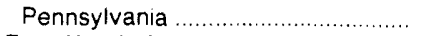 & 13,258 & 13,247 & 15,306 & 26,505 & 31,821 & -16.7 \\
\hline East North Central Total ............... & 51,006 & 48,329 & 54,242 & 99,335 & 106,475 & -6.7 \\
\hline 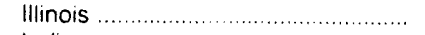 & 8,487 & 8,590 & 7,928 & 17,077 & 16,594 & 2.9 \\
\hline 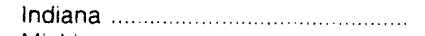 & $13,64 v$ & 13,330 & 15,524 & 26,976 & 30.940 & -12.8 \\
\hline 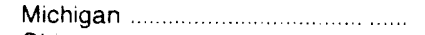 & 8,524 & 6,548 & 9,375 & 15,072 & 15,999 & -5.8 \\
\hline 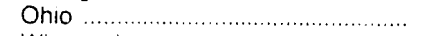 & 14,306 & 15,341 & 16,125 & 29,647 & 33,145 & -10.6 \\
\hline 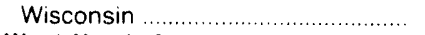 & 6,043 & 4,521 & 5,289 & 10,564 & 9,795 & 7.8 \\
\hline West North Central Total ................ & 27,000 & 29,098 & 28,288 & 56,097 & 57,727 & -2.8 \\
\hline 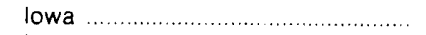 & 4,553 & 4,444 & 4,850 & 8.996 & 8,760 & 2.7 \\
\hline 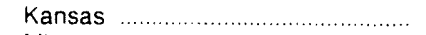 & 3,428 & 3,520 & 4,040 & 6.949 & 8,450 & -17.8 \\
\hline 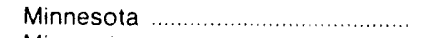 & 4,382 & 4,435 & 4,449 & 8,817 & 9,259 & -4.8 \\
\hline 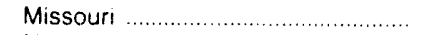 & 5,779 & 6,298 & 5,820 & 12,077 & 11,911 & 1.4 \\
\hline 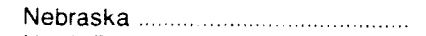 & 1,973 & 2,304 & 2,035 & 4,278 & 4,281 & -.1 \\
\hline 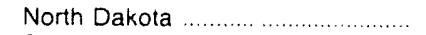 & 6.134 & 7,337 & 6,582 & 13,470 & 13,893 & -3.0 \\
\hline 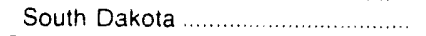 & 750 & 760 & 511 & 1,510 & 1,173 & 28.7 \\
\hline South Atlantic Total & 33,958 & 37,506 & 37,665 & 71,464 & 78,561 & -9.0 \\
\hline 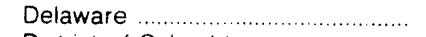 & 531 & 574 & 582 & 1,105 & 1.220 & -9.4 \\
\hline District of Columbia ...................... & 13 & 29 & 15 & 41 & 46 & -9.6 \\
\hline Florida & 5,820 & 5,565 & 6,188 & 11,384 & 12,593 & -9.6 \\
\hline 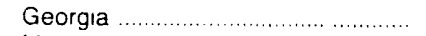 & 5,599 & 7,048 & 7,116 & 12,647 & 14,244 & -11.2 \\
\hline 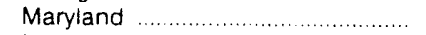 & 2,908 & 2.514 & 3,051 & 5,422 & 6,233 & -13.0 \\
\hline North Carolina & 4,772 & 5,723 & 5,711 & 10,495 & 12,061 & -13.0 \\
\hline South Carolina & 2,819 & 2,552 & 2,587 & 5,371 & 5,587 & -3.9 \\
\hline 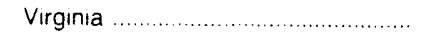 & 3,001 & 4,030 & 2,897 & 7,031 & 6,188 & 13.6 \\
\hline 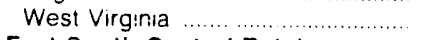 & 8.494 & 9,473 & 9,518 & 17,967 & 20,390 & -11.9 \\
\hline East South Central Total ............... & 22,052 & 22,095 & 24,513 & 44,147 & 49,211 & -10.3 \\
\hline Alabama & 7,110 & 6,827 & 6,947 & 13,937 & 13,897 & .3 \\
\hline 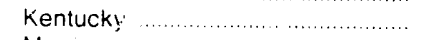 & 6,888 & 8,260 & 9,831 & 15,148 & 20,182 & -24.9 \\
\hline 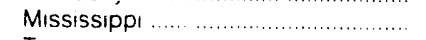 & 1.058 & 837 & 1,144 & 1,895 & 2,116 & -10.4 \\
\hline 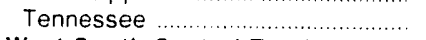 & 6,996 & 6,171 & 6.592 & 13,167 & 13,016 & 1.2 \\
\hline West South Central Total .................. & 31,221 & 34,230 & 31,073 & 65,451 & 62,857 & 4.1 \\
\hline Arkansas & 3,162 & 3,103 & 2,605 & 6,265 & 5,156 & 21.5 \\
\hline 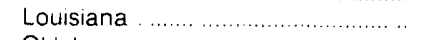 & 2.758 & 3,290 & 2,769 & 6.048 & 5,718 & 5.8 \\
\hline 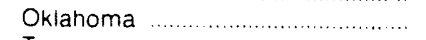 & 4,120 & 4,372 & 3,380 & 8.492 & 7,677 & 10.6 \\
\hline Texas $\ldots \ldots \ldots \ldots$ & 21.181 & 23,464 & 22,320 & 44,645 & 44,306 & .8 \\
\hline Mountain Total & 24,438 & 27,649 & 24,823 & 32,087 & 52,637 & -1.0 \\
\hline 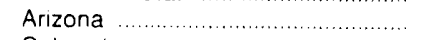 & 4,322 & 4,180 & 3,820 & 8.502 & 8,101 & 4.9 \\
\hline 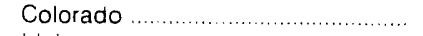 & 3,671 & 4,300 & 3,984 & 7,970 & 8,015 & -.6 \\
\hline 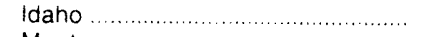 & 128 & 151 & 141 & 279 & 199 & 40.5 \\
\hline 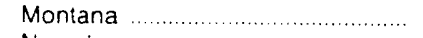 & 1,982 & 3,151 & 1,935 & 5,132 & 4,841 & 6.0 \\
\hline 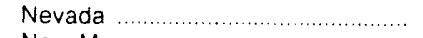 & 2.060 & 2,245 & 1,257 & 4,305 & 3,382 & 27.3 \\
\hline 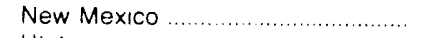 & 2,865 & 2,934 & 4,107 & 5.799 & 7,655 & -24.2 \\
\hline 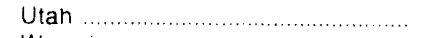 & 3,790 & 4,212 & 3,858 & 8,001 & 8,158 & -1.9 \\
\hline 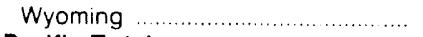 & 5,621 & 6,477 & 5.722 & 12,098 & 12,286 & -1.5 \\
\hline 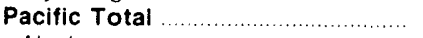 & 2,462 & 2,778 & 2,231 & 5,240 & 4,692 & 11.7 \\
\hline Alaska $\ldots \ldots \ldots \ldots$ & 180 & 232 & 164 & 412 & 390 & 5.7 \\
\hline Californa & 730 & 700 & 735 & 1,431 & 1,424 & .5 \\
\hline Oregon & 456 & 608 & - & 1,063 & 1 & NM \\
\hline Washington .......... & 1,096 & 1,238 & 1.332 & 2,334 & 2,878 & -18.9 \\
\hline 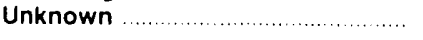 & 737 & 1,097 & 1,155 & 1,834 & 2,295 & -20.1 \\
\hline U.S. Total & 211,085 & 221,372 & 224,890 & 432,457 & 457,972 & -5.6 \\
\hline Canada ..... & 2,823 & 1,594 & 4,483 & 4,416 & 6.411 & -31.1 \\
\hline Overseas ${ }^{1}$ & 23.614 & 22,195 & 23.799 & 45,809 & 45,580 & .5 \\
\hline Foreign Total & 26,437 & 23,789 & 28,282 & 50,226 & 51,990 & -3.4 \\
\hline Grand Total & 237,522 & 245,161 & 253,172 & 482,683 & 509,962 & -5.3 \\
\hline
\end{tabular}

1 Also includes Mexico

- Quantity is less than 500 short tons or percent is less than 05

NM Not meaningful as value is greater than or equal to 500 .

Note: Total may not equal surn of components because of independent rounding.

Source: Energy Information Administration. Form ElA-6. "Coal Distribution Report." 


\section{Consuming Sectors}

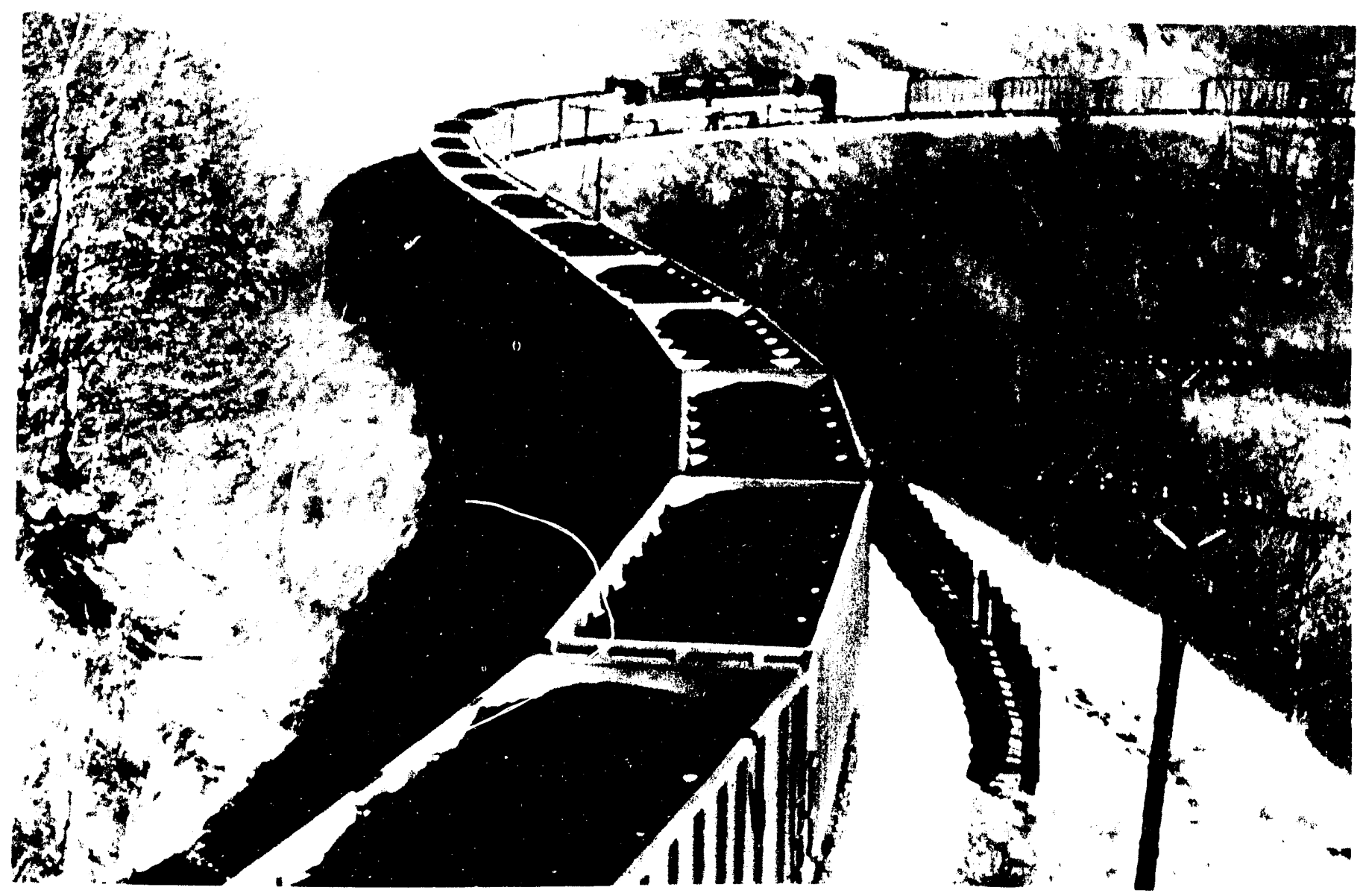

A train of aluminum railroad cars hauling coal from Kentucky to a power plant in Georgia. Aluminum cars are lighter than steel cars and can carry more coal without exceeding track limitations. 
Figure 2. Distribution of U.S. Coal by Consumer, January-June 1991

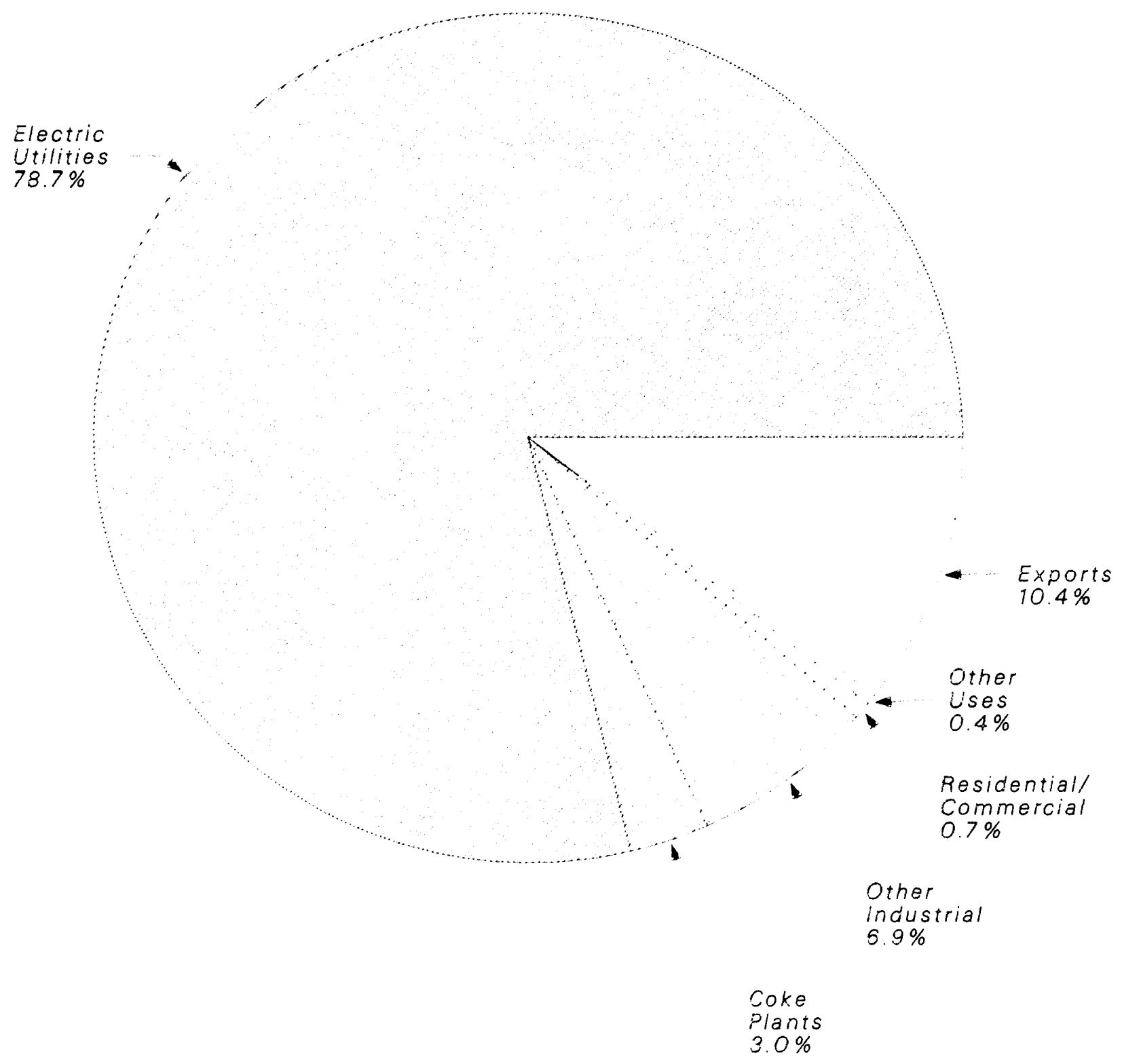

Source: Energy Information Administration, Form ElA-6. "Coal Distribution Report." 
Table 6. Domestic Distribution of U.S. Coal hy Origin and Consumer, January-June 1991

(Thousand Short Tons)

\begin{tabular}{|c|c|c|c|c|c|c|c|}
\hline $\begin{array}{l}\text { Coal-Producing State } \\
\text { and Region of Origin }\end{array}$ & $\begin{array}{l}\text { Electric } \\
\text { Utilities }\end{array}$ & $\begin{array}{l}\text { Coke } \\
\text { Plants }\end{array}$ & $\begin{array}{c}\text { Indus:rial } \\
\text { Plants } \\
\text { (Except Coke) }\end{array}$ & $\begin{array}{l}\text { Residential } \\
\text { and } \\
\text { Commercial }\end{array}$ & $\begin{array}{c}\text { Transporta- } \\
\text { tion }\end{array}$ & Unknown & Total \\
\hline Alabama .................. & 8,493 & 640 & 817 & 4 & . & 295 & 10.249 \\
\hline Alaska ................................ & 156 & - & - & 256 & - & - & 412 \\
\hline Arizona ............................ & 6,362 & - & - & - & - & - & 6,362 \\
\hline 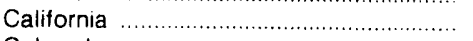 & - & - & 8 & - & - & - & 8 \\
\hline 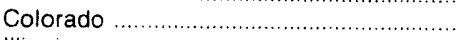 & 7,761 & 123 & 819 & 64 & · & 1 & 8,768 \\
\hline 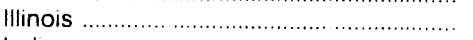 & 25,682 & 363 & 1,982 & 159 & - & 7 & 28.193 \\
\hline 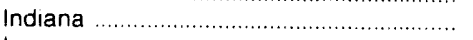 & 12,505 & - & 1,947 & 242 & - & 124 & 14,818 \\
\hline lowa & 174 & - & - & 1 & - & -. & 175 \\
\hline 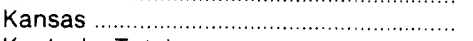 & 190 & - & 67 & 1 & - & - & 258 \\
\hline 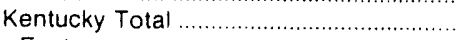 & 58,323 & 1,906 & 7.487 & 542 & $\cdot$ & 722 & 68,981 \\
\hline 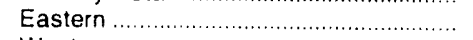 & 38,977 & 1,906 & 6,978 & 484 & $\cdot$ & 671 & 49,017 \\
\hline 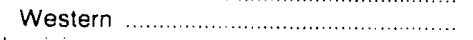 & 19,346 & - & 509 & 57 & - & 52 & 19,964 \\
\hline 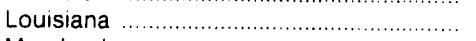 & 1,287 & - & - & - & - & - & 1,287 \\
\hline 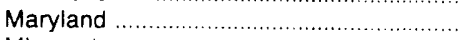 & 1,464 & - & 184 & 2 & - & 6 & 1.655 \\
\hline 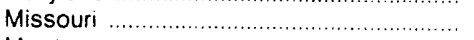 & 920 & - & 8 & 67 & - & 2. & 997 \\
\hline 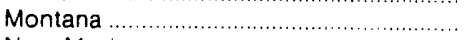 & 17.449 & - & 426 & 27 & $\ldots$ & 2 & 17.903 \\
\hline 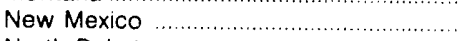 & 9,806 & - & 378 & 4 & .- & .. & 10.188 \\
\hline North Dakota & 11,566 & - & 2.969 & 70 & - & - & 14,604 \\
\hline Ohio & 13,937 & - & 1.221 & 171 & - & 57 & 15,386 \\
\hline 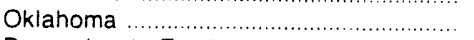 & 444 & - & 346 & * & - & 3 & 793 \\
\hline 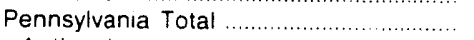 & 23,951 & 1,836 & 2,201 & 791 & - & 170 & 28,948 \\
\hline Anthracite & 490 & $\cdot$ & 292 & 425 & - & 20 & 1,227 \\
\hline 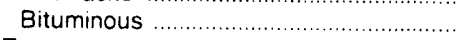 & 23.461 & 1,836 & 1.908 & 366 & - & 149 & 27.721 \\
\hline 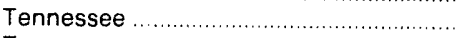 & 1.636 & - & 629 & 79 & - & 44 & 2,388 \\
\hline 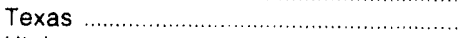 & 24.941 & - & 917 & - & - & - & 25,858 \\
\hline Utah & 7,358 & 301 & 1.787 & 269 & - & - & 9,715 \\
\hline 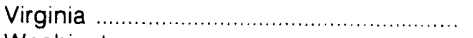 & 7.079 & 3.044 & 2.058 & 46 & $\cdot$ & 24 & 12,252 \\
\hline Washington & 2.193 & - & - & $*$ & - & - & 2.193 \\
\hline 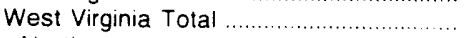 & 43,380 & 6,058 & 4.929 & 254 & 4 & 368 & 54.994 \\
\hline 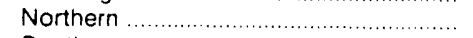 & 20,795 & - & 1,425 & 136 & - & 239 & 22.594 \\
\hline 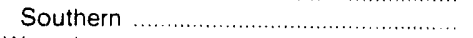 & 22.586 & 6,058 & 3.504 & 119 & $\epsilon_{4}$ & 130 & 32,400 \\
\hline Wyoming & 92,743 & - & 2.195 & 126 & - & 9 & 95.073 \\
\hline Appalachian Total & 138,917 & 13,486 & 19,017 & 1,830 & 5 & 1,635 & 174,889 \\
\hline Interior Total & 85,490 & 363 & 5,775 & 528 & - & 187 & 92,342 \\
\hline 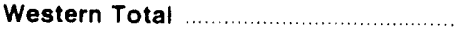 & 155,393 & 424 & 8,582 & 815 & + & 12 & 165,226 \\
\hline $\begin{array}{l}\text { East of the Miss. River } \\
\text { West of the Miss. River }\end{array}$ & $\begin{array}{l}196,450 \\
183,350\end{array}$ & $\begin{array}{r}13,848 \\
424\end{array}$ & $\begin{array}{r}23,454 \\
9,919\end{array}$ & $\begin{array}{r}2,289 \\
884\end{array}$ & 5 & $\begin{array}{r}1,818 \\
16\end{array}$ & $\begin{array}{l}237,864 \\
194,593\end{array}$ \\
\hline U.S. Total. & 379,800 & 14,272 & 33,374 & 3,173 & 5 & 1.834 & 432,457 \\
\hline
\end{tabular}

Note: Total may not equal sum of components because of independent rounding

Source: Energy Information Administration. Form ElA-6, "Coal Distribution Report. 
Table 7. Foreign Distribution of U.S. Coal by Origin and Consumer, January-June 1991

(Thousand Short Tons)

\begin{tabular}{|c|c|c|c|c|c|c|c|}
\hline & & & Canada & & & & \\
\hline $\begin{array}{l}\text { Coal-Producing State } \\
\text { and Region of Origin }\end{array}$ & $\begin{array}{l}\text { Electric } \\
\text { Utilities }\end{array}$ & $\begin{array}{l}\text { Coke } \\
\text { Plants }\end{array}$ & $\begin{array}{c}\text { Industrial } \\
\text { Plants } \\
\text { (Except Coke) }\end{array}$ & $\begin{array}{l}\text { Residential } \\
\text { and } \\
\text { Commercial }\end{array}$ & Total & Overseas ${ }^{1}$ & iotal \\
\hline Alabama ....... & - & - & - & - & - & 3,992 & 3,992 \\
\hline Alaska ................ & - & - & - & - & - & 340 & 340 \\
\hline Colorado & - & - & - & - & - & 332 & 332 \\
\hline Illinols & - & - & - & - & - & 400 & 400 \\
\hline Indiana & - & - & 3 & - & 3 & 59 & 62 \\
\hline Kentucky Total & 107 & 147 & 34 & - & 288 & 6.605 & 6.893 \\
\hline Eastern & 107 & 147 & 34 & - & 288 & 6,442 & 6,730 \\
\hline Western & - & - & - & - & - & 163 & 163 \\
\hline 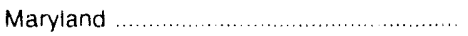 & - & - & - & - & - & 28 & 28 \\
\hline Montana & - & - & - & - & - & 148 & 148 \\
\hline Ohio & - & - & - & - & - & 14 & 14 \\
\hline 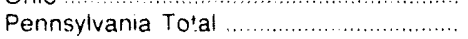 & 440 & 40 & 140 & 6 & 627 & 1,857 & 2,484 \\
\hline Anthracite & - & - & 132 & $\cdot$ & 132 & 14 & 145 \\
\hline Bituminous & 440 & 40 & 9 & 6 & 495 & 1,844 & 2.339 \\
\hline Tennessee & - & - & - & 4 & 4 & 2 & 6 \\
\hline Utah & - & - & - & - & - & 819 & 819 \\
\hline Virginia & 61 & 457 & 54 & - & 572 & 8,713 & 9,285 \\
\hline 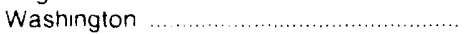 & - & - & - & - & - & 112 & 112 \\
\hline West Virgınıa Total & 1.874 & 1.025 & 23 & - & 2,923 & 21,926 & 24,849 \\
\hline Northern & 746 & - & 23 & - & 770 & 2,272 & 3.042 \\
\hline Southern & 1,128 & 1,025 & - & - & 2,153 & 19,654 & 21,807 \\
\hline Wyoming & - & - & - & - & - & 461 & 461 \\
\hline Appalachian Total & 2,482 & 1,669 & 252 & 10 & 4,413 & 42,975 & 47,388 \\
\hline Interior Total & - & - & 3 & - & 3 & 622 & 625 \\
\hline Western Total & - & - & - & - & - & 2,212 & 2,212 \\
\hline East of the Miss. River & 2,482 & 1,669 & 255 & 10 & 4,416 & 43,597 & 48,013 \\
\hline West of the Miss. River & - & - & - & - & - & 2,212 & 2,212 \\
\hline U.S. Total ........ & 2,482 & 1,669 & 255 & 10 & 4,416 & 45,809 & 50,226 \\
\hline
\end{tabular}

1 Also incli des Mexico.

- Quantity is less than 500 short tons or percent is less than 05 .

Note: Total may not equal sum of components because of irdependent rounding.

Source: Energy Information Administration, Form EIA-6. "Coal Distribution Report" 
Figure 3. Quarterly Domestic Coal Distribution By Consumer Category, 1983-1991

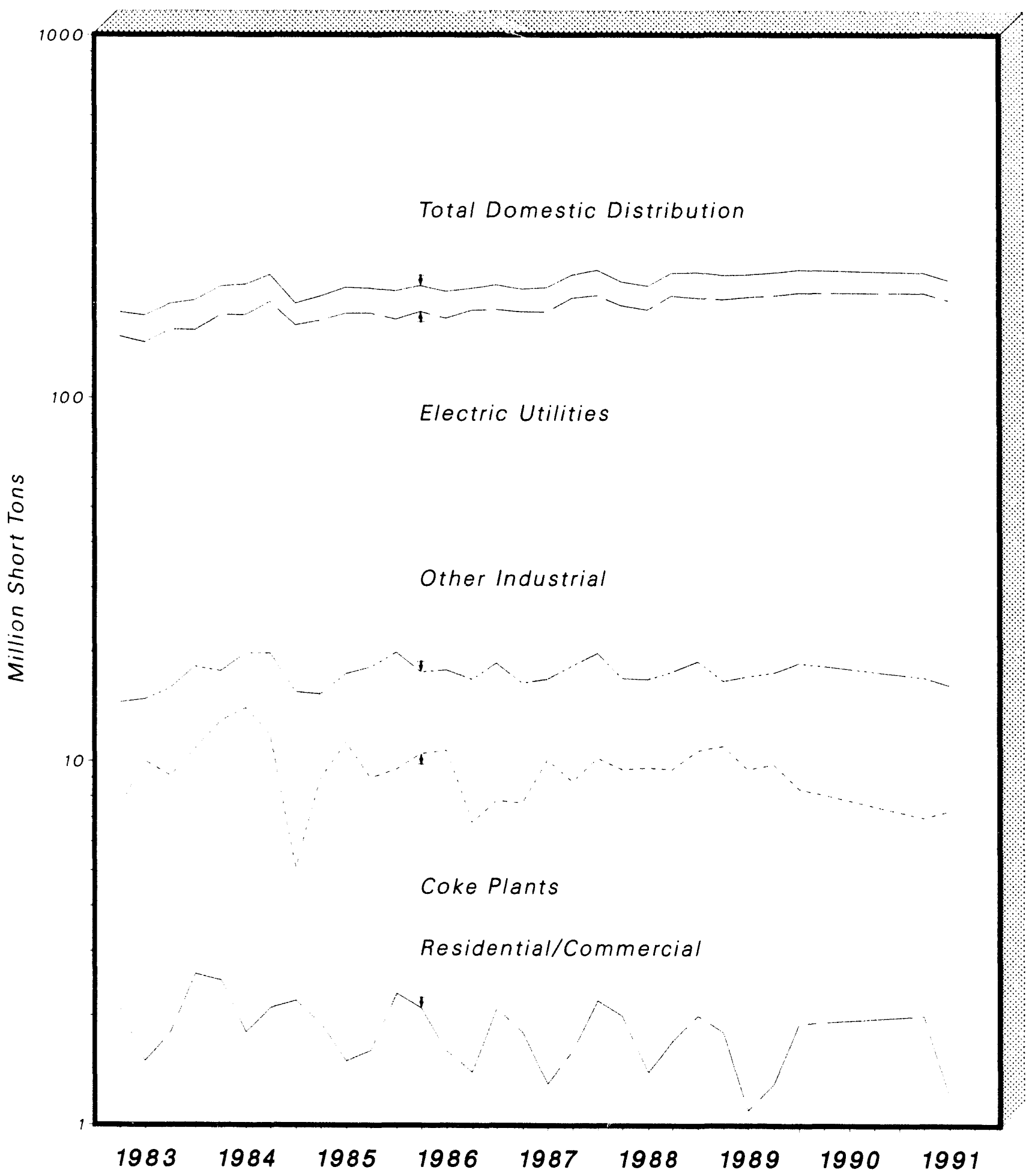

Note: Each increment represents end of quarter data.

Source: Energy Information Administration. Form ElA-6. "Coal Distribution Report." 
Table 8. Domestic Distribution of U.S. Coal by Destination and Consumer, January-June 1991

(Thousand Short Tons)

\begin{tabular}{|c|c|c|c|c|c|}
\hline $\begin{array}{l}\text { Census Division and } \\
\text { State of Destination }\end{array}$ & $\begin{array}{l}\text { Electric } \\
\text { Utilities }\end{array}$ & $\begin{array}{l}\text { Coke } \\
\text { Plants }\end{array}$ & $\begin{array}{c}\text { Industrial } \\
\text { Plants } \\
\text { (Except Coke) }\end{array}$ & $\begin{array}{c}\text { Residential } \\
\text { and Commercial }\end{array}$ & Total \\
\hline New England Total ................... & 2,763 & - & 45 & 23 & 2,830 \\
\hline Connecticut & 523 & - & - & 3 & 527 \\
\hline Mane & - & - & 10 & 3 & 12 \\
\hline 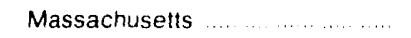 & 1.537 & - & 27 & 8 & 1,572 \\
\hline New Hampshire.................. & 703 & - & 1 & 6 & 710 \\
\hline Rhode Island $\ldots \ldots \ldots \ldots$ & - & - & - & 1 & 1 \\
\hline Vermont ......... & - & - & 7 & 2 & 9 \\
\hline Middle Atlantic Total & 26,999 & 3,478 & 2,689 & 807 & 33,973 \\
\hline New Jersey & 831 & - & 140 & 4 & 975 \\
\hline 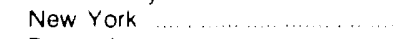 & 5,064 & 471 & 829 & 128 & 6,492 \\
\hline Pennsylvania $\ldots \ldots \ldots \ldots \ldots \ldots$ & 21.104 & 3.007 & 1,719 & 675 & 26,505 \\
\hline East North Central Total ........... & 84,077 & 5,808 & 8,776 & 674 & 99,335 \\
\hline Illinois & 13.961 & 766 & 2,249 & 102 & 17,077 \\
\hline Indiana $\ldots \ldots \ldots \ldots \ldots$ & 21,602 & 3.090 & 2.040 & 245 & 26,976 \\
\hline 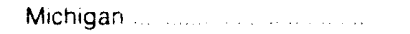 & 13,352 & 221 & 1.407 & 92 & 15,072 \\
\hline 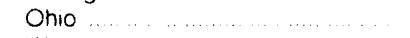 & 25.346 & 1.732 & 2,342 & 226 & 29.647 \\
\hline Wisconsin $\ldots \ldots \ldots \ldots$ & 9,816 & - & 738 & 9 & 10,564 \\
\hline West North Central Total $\ldots . . . . . .$. & 51,141 & - & 4,528 & 428 & 56,097 \\
\hline lowa $\ldots \ldots$ & 8.386 & - & 459 & 152 & 8.996 \\
\hline 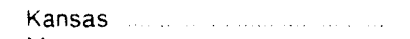 & 6.907 & - & 41 & $\cdot$ & 6.949 \\
\hline Mirnesota & 8.541 & - & 219 & 58 & 8.817 \\
\hline Missouri $\ldots \ldots \ldots$ & 11.409 & - & 533 & 136 & 12,077 \\
\hline Nebraska $\ldots \ldots \ldots \ldots$ & 4.206 & - & 65 & 7 & 4,278 \\
\hline North Dakota ... ... ............. & 10.287 & - & 3,109 & 74 & 13,470 \\
\hline South Dakota & 1.406 & - & 103 & 2 & 1.510 \\
\hline South Atlantic Total & 60,927 & 2,773 & 7,439 & 321 & 171,464 \\
\hline Delaware $\ldots \ldots$ & 1.010 & - & 91 & 4 & 1,105 \\
\hline District of Columbia $\ldots$ & - & - & - & 41 & 41 \\
\hline Florida $\ldots \ldots$ & 11.017 & - & 367 & $\cdot$ & 11.384 \\
\hline Georgıa ... . . . . . . . & 11.629 & - & 1.014 & 4 & 12,647 \\
\hline Maryland $\ldots \ldots \ldots \ldots$ & 4.529 & 267 & 614 & 13 & 5.422 \\
\hline 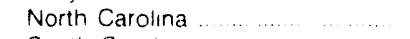 & 9,211 & - & 1,238 & 47 & 10,495 \\
\hline South Carolına $\ldots \ldots \ldots \ldots \ldots$ & 4,297 & - & 1,058 & 16 & 5.371 \\
\hline 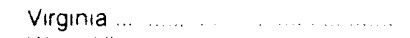 & 4,658 & 480 & 1.830 & 64 & 17.031 \\
\hline 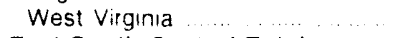 & 14.578 & 2.026 & 1,226 & 132 & 117,967 \\
\hline East South Central Total & 38,397 & 1,544 & 3,966 & 240 & 44,147 \\
\hline Alabama & 11.599 & 1.139 & 1,188 & 10 & 13,937 \\
\hline 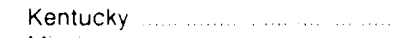 & 13.711 & 405 & 931 & 102 & 115,148 \\
\hline Mississippi _... ............... ... & 1,823 & - & 72 & - & 1,895 \\
\hline Tennessee $\ldots . . . \ldots \ldots \ldots \ldots$ & 11,264 & - & 1.776 & 128 & 13,167 \\
\hline West South Central Total .......... & 63,340 & - & 2,104 & 6 & 65,451 \\
\hline Arkansas & 6.156 & .. & 110 & * & 6,265 \\
\hline 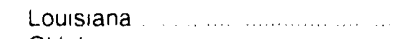 & 5.792 & - & 256 & - & 6,048 \\
\hline Oklahoma $\ldots \ldots \ldots \ldots \ldots \ldots \ldots$ & 8.176 & - & 315 & * & 8,492 \\
\hline Texas $\ldots \ldots \ldots$ & 43.216 & - & 1.423 & 6 & 44,645 \\
\hline Mountain Total $\ldots \ldots \ldots$ & 48,762 & 669 & 2,341 & 314 & 152,087 \\
\hline Arizona $\ldots \ldots \ldots \ldots$ & 8.133 & - & 369 & $*$ & 8,502 \\
\hline 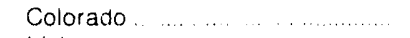 & 7.613 & - & 329 & 28 & 17,970 \\
\hline 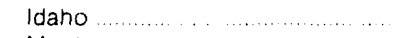 & - & - & 243 & 36 & 279 \\
\hline 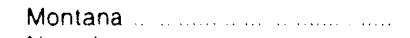 & 4.982 & - & 130 & 21 & 5,132 \\
\hline Nevada $\ldots \ldots \ldots \ldots \ldots$ & 4.190 & - & 113 & 1 & 4,305 \\
\hline New Mexico $\ldots \ldots \ldots$ & 5.774 & - & 22 & 4 & 5,799 \\
\hline Utah $\ldots \ldots \ldots \ldots \ldots$ & 6.891 & 669 & 279 & 161 & 8,001 \\
\hline Wyoming $\ldots \ldots \ldots \ldots \ldots \ldots$ & 11.179 & - & 856 & 63 & 12,098 \\
\hline Pacific Total $\ldots \ldots \ldots$ & 3,394 & - & 1,486 & 360 & 5,240 \\
\hline 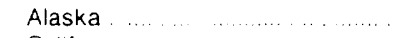 & 156 & - & - & 256 & 412 \\
\hline 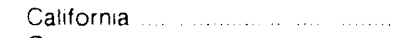 & - & - & 1.388 & 43 & 1.431 \\
\hline Oregon $\ldots \ldots \ldots \ldots \ldots \ldots \ldots$ & 1.045 & - & 18 & 1 & 1,063 \\
\hline 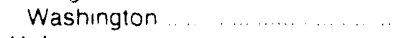 & 2.193 & - & 81 & 60 & 2,334 \\
\hline Unknown $\ldots \ldots \ldots$ & - & - & - & - & 21,834 \\
\hline U.S. Total & 379,800 & 14,272 & 33,374 & 3,173 & ${ }^{3} 432,457$ \\
\hline
\end{tabular}

1 Includes distribution to the transportation sector

2 Includes distribution to unknown consumers.

3 Includes distribution to both the transportation sector and unknown consumers.

Quantity is less than 500 short tons or percent is less than 05

Note. Total may not equal surr of components vecause of independent rounding.

Source Energy Information Administration. Form ElA.6 "Coal Distribution Report." 
Table 9. Domestic Distribution of U.S. Coal to Electric Utility Plants by Origin (Thousand Short Tons)

\begin{tabular}{|c|c|c|c|c|c|c|}
\hline $\begin{array}{l}\text { Coal-Producing State } \\
\text { and Region of Origin }\end{array}$ & $\begin{array}{l}\text { April- } \\
\text { June } \\
1991\end{array}$ & $\begin{array}{c}\text { January- } \\
\text { March } \\
1991\end{array}$ & $\begin{array}{l}\text { April- } \\
\text { June } \\
1990\end{array}$ & 1991 & ar to Dat & $\begin{array}{l}\text { Percent } \\
\text { Change }\end{array}$ \\
\hline Alabama & ... & 205 & & & & 46 \\
\hline $\begin{array}{l}\text { Alabama } \\
\text { Alaska }\end{array}$ & $\begin{array}{r}4,287 \\
72\end{array}$ & $\begin{array}{r}4,205 \\
84\end{array}$ & $\begin{array}{r}3.919 \\
61\end{array}$ & $\begin{array}{r}8,493 \\
156\end{array}$ & $\begin{array}{r}8.120 \\
141\end{array}$ & 11.0 \\
\hline 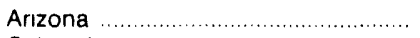 & 3,202 & 3.160 & 2.106 & 6,362 & 5.138 & 23.8 \\
\hline Colorado & 3,896 & 3.864 & 3,820 & 7,761 & 7,832 & -.9 \\
\hline 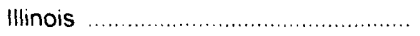 & 12,785 & 12,897 & 14.213 & 25,682 & $28,6,8$ & -10.2 \\
\hline 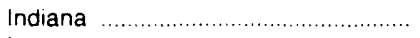 & 6,151 & 6,354 & 7,759 & 12,505 & 15.397 & -18.8 \\
\hline 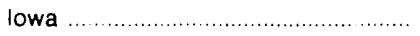 & 80 & 95 & 88 & 174 & 183 & -4.9 \\
\hline 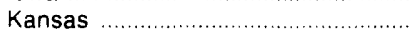 & 82 & 108 & 139 & 190 & 342 & -44.6 \\
\hline 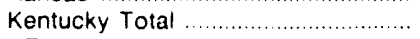 & 27,917 & 30,406 & 33,280 & 58,323 & 67.169 & -13.2 \\
\hline 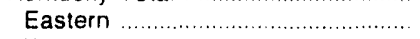 & 18,551 & 20,426 & 22.650 & 38,977 & 45.524 & -14.4 \\
\hline 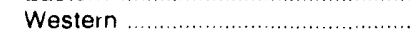 & 9,367 & 9,979 & 10,630 & 19,346 & 21,645 & -10.6 \\
\hline 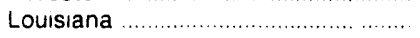 & 581 & 707 & 854 & 1.287 & 1,502 & -14.3 \\
\hline 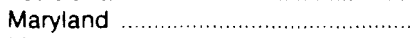 & 657 & 807 & 555 & 1,464 & 1,202 & 21.8 \\
\hline 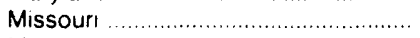 & 432 & 489 & 601 & 920 & 1,263 & -27.1 \\
\hline 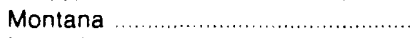 & 8.762 & 8,686 & 8,895 & 17,449 & 17.831 & -2.1 \\
\hline 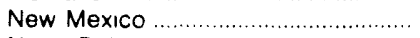 & 4,924 & 4,882 & 6,161 & 9,806 & 11.607 & -15.5 \\
\hline 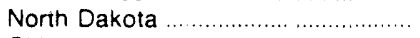 & 5,174 & 6,392 & 5,724 & 11,566 & 12,129 & -4.6 \\
\hline 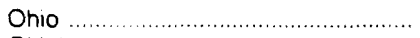 & 7,206 & 6,731 & 7.357 & 13.937 & 15,682 & -11.1 \\
\hline Oklahoma & 255 & 189 & 260 & 444 & 613 & -27.6 \\
\hline 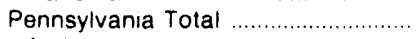 & 12,018 & 11.933 & 13.046 & 23,951 & 26,309 & -9.0 \\
\hline Anthracite & 283 & 206 & 252 & 490 & 358 & 36.7 \\
\hline 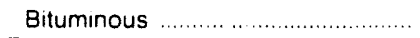 & 11,735 & 11,727 & 12.793 & 23,461 & 25.951 & -9.6 \\
\hline 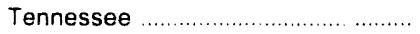 & 840 & 795 & 1,105 & 1,636 & 2,370 & -31.0 \\
\hline 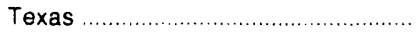 & 12,679 & 12.262 & 12,661 & 24,941 & 24,988 & -2 \\
\hline 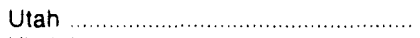 & 3,369 & 3,989 & 3,620 & 7,358 & 7.565 & -2.7 \\
\hline 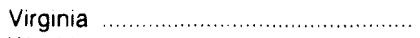 & 3,246 & 3,833 & 3,936 & 7,079 & 7,854 & -9.9 \\
\hline (. & 1.030 & 1,162 & 1,219 & 2,193 & 2,445 & -10.3 \\
\hline 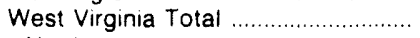 & 22.087 & 21.294 & 21,856 & 43,380 & 44,950 & -3.5 \\
\hline 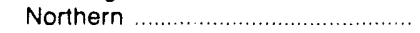 & 10,216 & 10,579 & 11,225 & 20,795 & 23,519 & -11.6 \\
\hline Southern & 11,870 & 10,715 & 10,631 & 22.586 & 21,431 & 5.4 \\
\hline 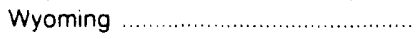 & 43.967 & 48,777 & 41,944 & 92,743 & 85,534 & 8.4 \\
\hline 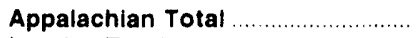 & 68,892 & 70,024 & 74,423 & 138,917 & 152,011 & -8.6 \\
\hline 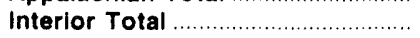 & 42,411 & 43,079 & 47,204 & 85,490 & 94,542 & -9.6 \\
\hline 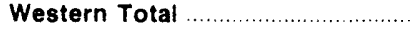 & 74,396 & 80,997 & 73,549 & 155,393 & 150,221 & 3.4 \\
\hline $\begin{array}{l}\text { East of the Miss. River } \ldots \ldots \ldots \ldots \ldots \\
\text { West of the Miss. River } \ldots \ldots \ldots \ldots \ldots \ldots\end{array}$ & $\begin{array}{l}97,195 \\
88,504\end{array}$ & $\begin{array}{l}99,254 \\
94,846\end{array}$ & $\begin{array}{r}107,024 \\
88,151\end{array}$ & $\begin{array}{l}196,450 \\
183,350\end{array}$ & $\begin{array}{l}217,662 \\
179,112\end{array}$ & $\begin{array}{r}-9.7 \\
2.4\end{array}$ \\
\hline 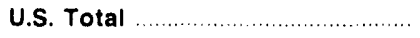 & 185,699 & 194,101 & 195,176 & 379,800 & 396,774 & -4.3 \\
\hline
\end{tabular}

Note: Total may not equal sum of romponents because of independent rounding

Source: Energy Information Administration. Form ElA-6, "Coal Distribution Report." 
Table 10. Domestic Distribution of U.S. Coal to Electric Utility Plants by Destination

(Thousand Short Tons)

\begin{tabular}{|c|c|c|c|c|c|c|}
\hline $\begin{array}{l}\text { Census Division and } \\
\text { State of Destination }\end{array}$ & $\begin{array}{l}\text { April- } \\
\text { June } \\
1991\end{array}$ & $\begin{array}{l}\therefore \text { auidary- } \\
\text { March } \\
1991\end{array}$ & $\begin{array}{l}\text { April- } \\
\text { June } \\
1990\end{array}$ & 1991 & ar to Dat & $\begin{array}{l}\text { Percent } \\
\text { Change }\end{array}$ \\
\hline New England Total ........................ & 1,243 & 1,520 & 1,363 & 2,763 & 2,847 & -2.9 \\
\hline Connecticut & 240 & 283 & 436 & 523 & 681 & -23.1 \\
\hline 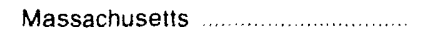 & 668 & 869 & 791 & 1.537 & 1.742 & -11.8 \\
\hline New Hampshire .......... & 335 & 368 & 137 & 703 & 424 & 65.7 \\
\hline Middle Atlantic Total ........................ & 13,527 & 13,472 & 15,079 & 26,999 & 30,592 & -11.7 \\
\hline 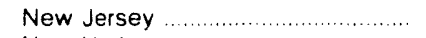 & 346 & 485 & 487 & 831 & 1,064 & -21.9 \\
\hline New York & 2.579 & 2,485 & 2,972 & 5,064 & 5,860 & -13.6 \\
\hline 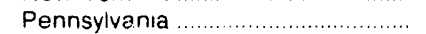 & 10,602 & 10,502 & 11,620 & 21,104 & 23,668 & -10.8 \\
\hline East North Central Total ................. & 43,405 & 40,672 & 45,138 & 84,077 & 88,479 & -5.0 \\
\hline 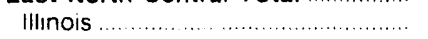 & 7,115 & 6.846 & 6,737 & 13,961 & 13,933 & 2 \\
\hline 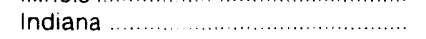 & 10,812 & 10,790 & 12,478 & 21.602 & 24.459 & -11.7 \\
\hline Michigan & 7,647 & 5,705 & 7,924 & 13,352 & 13,621 & -2.0 \\
\hline Ohio & 12,222 & 13,125 & 13,119 & 25,346 & 27,344 & -7.3 \\
\hline 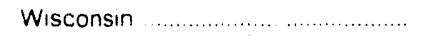 & 5,610 & 4.206 & 4,879 & 9,816 & 9,122 & 7.6 \\
\hline West North Central Total ................ & 24,451 & 26,691 & 25,731 & 51,141 & 52,738 & -3.0 \\
\hline lowa & 4,106 & 4,280 & 4.374 & 8,386 & 8.071 & 3.9 \\
\hline Kansas & 3.401 & 3.507 & 4.012 & 6,907 & 8.402 & -17.8 \\
\hline (n) & 4,309 & 4,232 & 4,124 & 8,541 & 8,654 & -1.3 \\
\hline 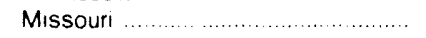 & 5,463 & 5.946 & 5,436 & 11,409 & 11,150 & 2.3 \\
\hline 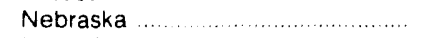 & 1,943 & 2,263 & 2,016 & 4,206 & 4,221 & -.4 \\
\hline 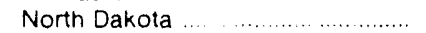 & 4.544 & 5,744 & 5,327 & 10,287 & 11,178 & -8.0 \\
\hline South Dakota & 687 & 719 & 442 & 1,406 & 1,062 & 32.4 \\
\hline South Atlantic Total $\ldots \ldots \ldots \ldots \ldots \ldots$ & 28,961 & 31,966 & 32,176 & 60,927 & 67,379 & -9.6 \\
\hline Delaware & 479 & 532 & 538 & 1,010 & 1,134 & -10.9 \\
\hline 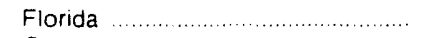 & 5,645 & 5,372 & 5.966 & 11,017 & 12.076 & -8.8 \\
\hline 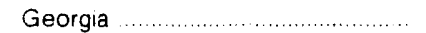 & 5,151 & 6,478 & 6,375 & 11,629 & 13,021 & -10.7 \\
\hline . ............................... & 2,441 & 2,088 & 2,484 & 4.529 & 5.000 & -9.4 \\
\hline 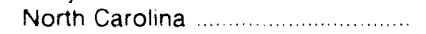 & 4,182 & 5,028 & 4,919 & 9,211 & 10.349 & -11.0 \\
\hline 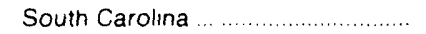 & 2.260 & 2,037 & 2,074 & 4,297 & 4,408 & -2.5 \\
\hline . . .................. & 2,019 & 2,639 & 1,716 & 4,658 & 3,873 & 20.3 \\
\hline 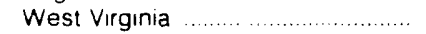 & 6,785 & 7,792 & 8,104 & $\uparrow 4,578$ & 17,517 & -16.8 \\
\hline East South Central Total ................ & 19,247 & 19,150 & 21,329 & 38,397 & 42,627 & -9.9 \\
\hline Alabama & 5,952 & 5.647 & 5.509 & 11,599 & 10.970 & 5.7 \\
\hline 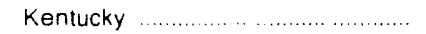 & 6,258 & 7.453 & 8,902 & 13.711 & 18,337 & -25.2 \\
\hline 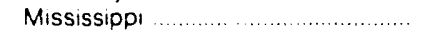 & 1.029 & 794 & 1,104 & 1,823 & 2,036 & -10.4 \\
\hline (2, & 6,008 & 5,255 & 5,814 & 11.264 & 11,284 & -.2 \\
\hline West South Central Total ............... & 30,435 & 32,905 & 29,679 & 63,340 & 59,653 & 6.2 \\
\hline Arkansas & 3,107 & 3,049 & 2,547 & 6,156 & 5,044 & 22.0 \\
\hline 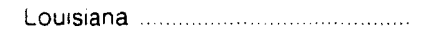 & 2,638 & 3.154 & 2.602 & 5.792 & 5,381 & 7.6 \\
\hline 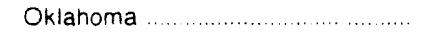 & 3,954 & 4,223 & 3,233 & 8,176 & 7.414 & 10.3 \\
\hline Texas & 20.736 & 22,480 & 21,297 & 43.216 & 41,813 & 3.4 \\
\hline Mountain Total ..... & 22,873 & 25,889 & 23,336 & 48,762 & 49,572 & -1.6 \\
\hline$\ldots \ldots \ldots \ldots \ldots \ldots \ldots \ldots \ldots \ldots \ldots \ldots \ldots \ldots \ldots \ldots \ldots \ldots$ & 4,142 & 3.991 & 3,662 & 8,133 & 7,762 & 4.8 \\
\hline 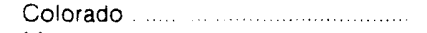 & 3,512 & 4,101 & 3,836 & 7,613 & 7.670 & -.7 \\
\hline Montana & 1.917 & 3.065 & 1,888 & 4.982 & 4.715 & 5.6 \\
\hline 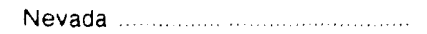 & 2,003 & 2,188 & 1,206 & 4.190 & 3,300 & 27.0 \\
\hline 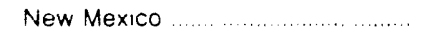 & 2,855 & 2,919 & 4,097 & 5,774 & 7.633 & -24.4 \\
\hline Utah $\ldots \ldots$ & 3.241 & 3,650 & 3.345 & 6.891 & 7.093 & -2.8 \\
\hline Wyoming & 5,204 & 5.975 & 5,303 & 11.179 & 11,398 & -1.9 \\
\hline Pacific Total $\ldots \ldots \ldots$ & 1,558 & 1,836 & 1,345 & 3,394 & 2,888 & 17.5 \\
\hline 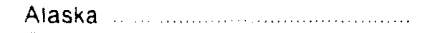 & 72 & 84 & 61 & 156 & 141 & 11.0 \\
\hline Oregon $\ldots \ldots \ldots \ldots \ldots \ldots \ldots \ldots$ & 455 & 589 & - & 1,045 & - & - \\
\hline 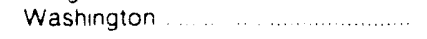 & 1.030 & 1,162 & 1,285 & 2,193 & 2.747 & -20.2 \\
\hline U.S. Total & 185,699 & 194,101 & 195,176 & 379,800 & 396,774 & -4.3 \\
\hline
\end{tabular}

Note: Total may not equal sum of components because of independent rounding.

Source Energy Information Administration, Form EIA-6, "Coal Distribution Report." 
Table 11. Domestic Distribution of U.S. Coal to Coke Plants by Origin (Thousand Short Tons)

\begin{tabular}{|c|c|c|c|c|c|c|}
\hline $\begin{array}{l}\text { Coal-Producing State } \\
\text { and Pegion of Origin }\end{array}$ & $\begin{array}{l}\text { April- } \\
\text { June } \\
1991\end{array}$ & $\begin{array}{c}\text { January- } \\
\text { March } \\
1991\end{array}$ & $\begin{array}{c}\text { April: } \\
\text { June } \\
1990\end{array}$ & 1991 & ar to Dat & $\begin{array}{l}\text { Percent } \\
\text { Change }\end{array}$ \\
\hline Alabama & 297 & 344 & 600 & 640 & 1.113 & -42.5 \\
\hline Colorado & 40 & 83 & 146 & 123 & 318 & $-6,3$ \\
\hline lllinols & 169 & 194 & 309 & 363 & 720 & -49.6 \\
\hline Indiana & - & - & - & - & 24 & - \\
\hline 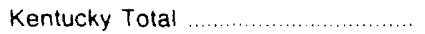 & 969 & 937 & 1.351 & 1,906 & 2,729 & -30.2 \\
\hline 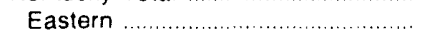 & 969 & 937 & 1.351 & 1,906 & 2,714 & -29.8 \\
\hline 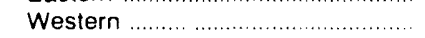 & - & - & - & .. & 15 & - \\
\hline 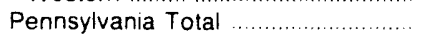 & 945 & 891 & 1.242 & 1,836 & 2,866 & -35.9 \\
\hline Anthracite & - & 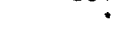 & 5 & 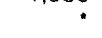 & 28 & -99.7 \\
\hline 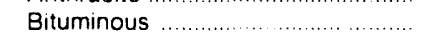 & 945 & 891 & 1.237 & 1,836 & 2,838 & -35.3 \\
\hline Utah & 147 & 153 & 163 & 301 & 322 & -6.5 \\
\hline Virginia & 1,520 & 1.524 & 2,177 & 3.044 & 4,396 & -30.7 \\
\hline West Virgina Total & 3,215 & 2,843 & 4,357 & 6.058 & 8,558 & -29.2 \\
\hline 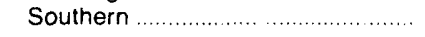 & 3,215 & 2,843 & 4,357 & 6.058 & 8,558 & -29.2 \\
\hline Appalachian Total $\ldots \ldots \ldots \ldots \ldots \ldots \ldots \ldots \ldots \ldots \ldots$ & 6.946 & 6,539 & 9,726 & 13,486 & 19,647 & -31.4 \\
\hline Interior Total & 169 & 194 & 309 & 363 & 758 & -52.2 \\
\hline 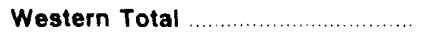 & 187 & 236 & 309 & 424 & 640 & -33.8 \\
\hline $\begin{array}{l}\text { East of the Miss. River } \\
\text { West of the Miss. River } \ldots \ldots \ldots \ldots \ldots \ldots \ldots \ldots \ldots\end{array}$ & $\begin{array}{r}7,115 \\
187\end{array}$ & $\begin{array}{r}6,733 \\
236\end{array}$ & $\begin{array}{r}10,036 \\
309\end{array}$ & $\begin{array}{r}13,848 \\
424\end{array}$ & $\begin{array}{r}20,405 \\
640\end{array}$ & $\begin{array}{l}-32.1 \\
-33.8\end{array}$ \\
\hline U.S. Total & 7,302 & 6,970 & 10,345 & 14,272 & 21,045 & -32.2 \\
\hline
\end{tabular}

- Quantity is less than 500 short tons or pe:cent is less than 05

Note: Total may not equal sum of components because of independent rounding.

Source: Energy Information Administration. Form EIA-6, "Coal Distribution Report."

Table 12. Domestic Distribution of U.S. Coal to Coke Plants by Destination (Thousand Short Tons)

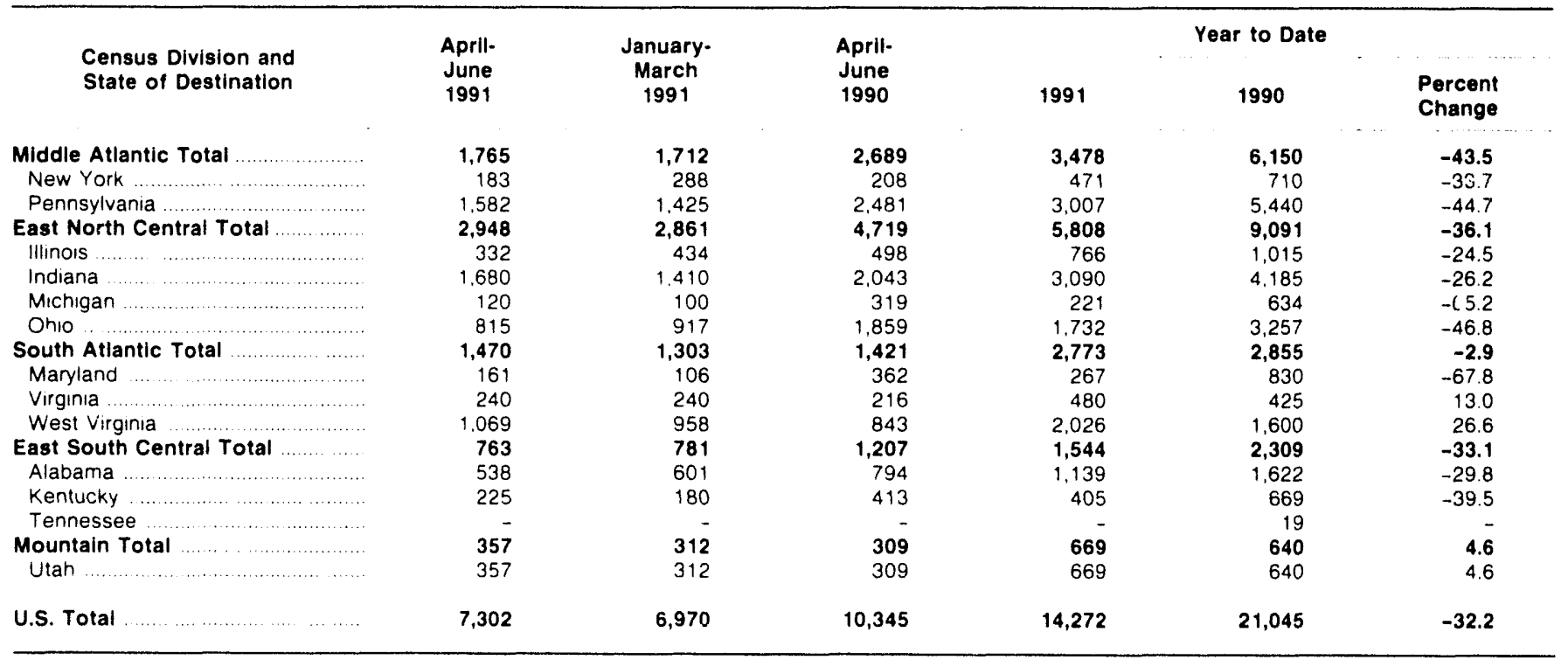

Note: Total may not equal sum of components because of independent rounding.

Source: Energy Information Administration. Form ElA-6, "Coal Distribution Report" 


\section{Table 13. Domestic Distribution of U.S. Coal to Industrial Plants (Except Coke) by Origin (Thousand Short Tons)}

\begin{tabular}{|c|c|c|c|c|c|c|}
\hline $\begin{array}{l}\text { Coal-Producing State } \\
\text { and Region of Origin }\end{array}$ & $\begin{array}{l}\text { April- } \\
\text { June } \\
1991\end{array}$ & $\begin{array}{l}\text { January- } \\
\text { March } \\
1991\end{array}$ & $\begin{array}{l}\text { Ipril- } \\
\text { ne } \\
1.70\end{array}$ & 1991 & ar to Date & $\begin{array}{l}\text { Percent } \\
\text { Changc }\end{array}$ \\
\hline Alabama & 426 & 390 & 536 & 817 & 1,101 & -25.8 \\
\hline California & 8 & - & 11 & 8 & 44 & -81.7 \\
\hline Coloradc & 443 & 376 & 436 & 819 & 888 & -7.7 \\
\hline 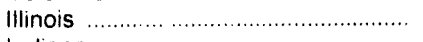 & 943 & 1,039 & 833 & 1.982 & 1,886 & 5.1 \\
\hline 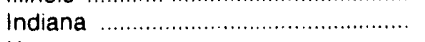 & 1.014 & 933 & 1,018 & 1,947 & 2,024 & -3.8 \\
\hline 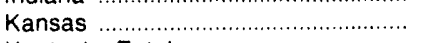 & 40 & 27 & 48 & 67 & 76 & -11.3 \\
\hline 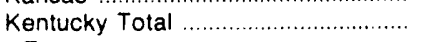 & 3,854 & 3,633 & 3,779 & 7,487 & 7,761 & -3.5 \\
\hline 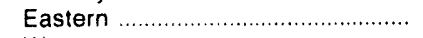 & 3.605 & 3,373 & 3,342 & 6,978 & 6.940 & 6 \\
\hline 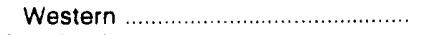 & 249 & 260 & 436 & 509 & 821 & -38.0 \\
\hline 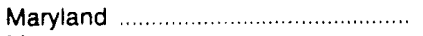 & 83 & 102 & 76 & 184 & 181 & 1.8 \\
\hline 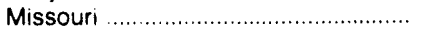 & 3 & 5 & 11 & 8 & 22 & -65.4 \\
\hline 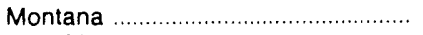 & 19 & 407 & 10 & 426 & 370 & 15.0 \\
\hline 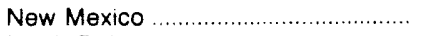 & 174 & 204 & 166 & 378 & 332 & 13.9 \\
\hline 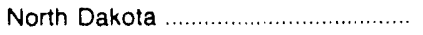 & 1,576 & 1,393 & 1,246 & 2.969 & 2,527 & 17.5 \\
\hline Ohio & 518 & 703 & 641 & 1,221 & 1,352 & -9.7 \\
\hline 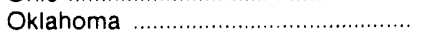 & 178 & 168 & 154 & 346 & 278 & 24.5 \\
\hline 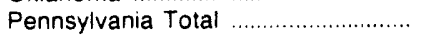 & 1,146 & 1,054 & 1.224 & 2,201 & 2.341 & -6.0 \\
\hline Anthracite & 127 & 165 & 102 & 292 & 215 & 35.7 \\
\hline 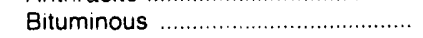 & 1,019 & 889 & 1,122 & 1,908 & 2,125 & -10.2 \\
\hline 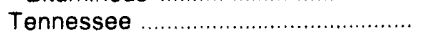 & 261 & 368 & 545 & 629 & 916 & -31.4 \\
\hline Texas & 189 & 728 & 708 & 917 & 1,830 & -49.9 \\
\hline 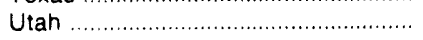 & 933 & 855 & 894 & 1,787 & 1.764 & 1.3 \\
\hline 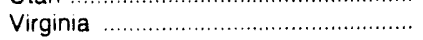 & 963 & 1,095 & 878 & 2,058 & 1.811 & 13.7 \\
\hline 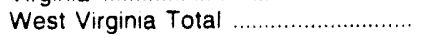 & 2,355 & 2,574 & 2,509 & 4.929 & 4,987 & -1.2 \\
\hline 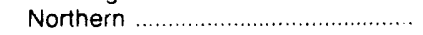 & 759 & 666 & 792 & 1,425 & 1.556 & -8.4 \\
\hline 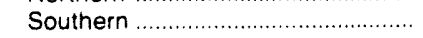 & 1,596 & 1,908 & 1,717 & 3,504 & 3.432 & 2.1 \\
\hline Wyoming & 1,052 & 1,143 & 1,219 & 2.195 & 2,157 & 1.8 \\
\hline $\begin{array}{l}\text { Appalachian Total } \\
\text { Interior Total } \ldots \ldots \ldots \ldots \ldots \ldots \ldots \ldots \ldots \ldots \ldots \ldots \ldots \ldots \ldots \ldots \ldots \ldots \ldots \ldots \ldots \ldots \ldots \ldots \ldots \ldots \ldots \ldots \ldots \\
\text { Western Total }\end{array}$ & $\begin{array}{l}9,357 \\
2,615 \\
4,205\end{array}$ & $\begin{array}{l}9,660 \\
3,160 \\
4,378\end{array}$ & $\begin{array}{l}9,751 \\
3,206 \\
3,983\end{array}$ & $\begin{array}{r}19,017 \\
5,775 \\
8,582\end{array}$ & $\begin{array}{r}19,628 \\
6,936 \\
8,082\end{array}$ & $\begin{array}{r}-3.1 \\
-16.7 \\
6.2\end{array}$ \\
\hline $\begin{array}{l}\text { East of the Miss. River } \ldots \ldots \ldots \ldots \ldots \ldots \\
\text { West of the Miss. River } \ldots \ldots \ldots \ldots \ldots \ldots\end{array}$ & $\begin{array}{r}11,563 \\
4,614\end{array}$ & $\begin{array}{r}11,891 \\
5,305\end{array}$ & $\begin{array}{r}12,037 \\
4,903\end{array}$ & $\begin{array}{r}23,454 \\
9,919\end{array}$ & $\begin{array}{l}24,359 \\
10,288\end{array}$ & $\begin{array}{l}-3.7 \\
-3.6\end{array}$ \\
\hline U.S. Total & 16,177 & 17,197 & 16,940 & 33,374 & 34,647 & -3.7 \\
\hline
\end{tabular}

Note: Total may not equal sum of components because of independent rounding.

Source: Energy Information Administration, Form EIA-6, "Coal Distribution Report." 
Table 14. Domestic Distribution of U.S. Coal to Industrial Plants (Except Coke) by Destination

(Thousand Short Tons)

\begin{tabular}{|c|c|c|c|c|c|c|}
\hline \multirow{2}{*}{$\begin{array}{l}\text { Census Division and } \\
\text { State of Destination }\end{array}$} & \multirow{2}{*}{$\begin{array}{l}\text { April. } \\
\text { June } \\
1991\end{array}$} & \multirow{2}{*}{$\begin{array}{c}\text { January- } \\
\text { March } \\
1991\end{array}$} & \multirow{2}{*}{$\begin{array}{l}\text { April- } \\
\text { June } \\
1990\end{array}$} & \multicolumn{3}{|c|}{ Year to Date } \\
\hline & & & & 1991 & 1990 & $\begin{array}{l}\text { Percent } \\
\text { Change }\end{array}$ \\
\hline New England Total $\ldots \ldots \ldots \ldots$ & 27 & 17 & 32 & 45 & 110 & -59.6 \\
\hline Connecticut & - & - & • & - & 1 & - \\
\hline Maine & 10 & - & 11 & 10 & 52 & -81.2 \\
\hline 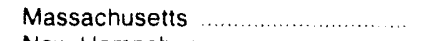 & 10 & 17 & 18 & 27 & 40 & -33.7 \\
\hline 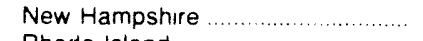 & 1 & $\cdot$ & 3 & 1 & 16 & -93.3 \\
\hline Rhode Island & - & - & • & - & • & - \\
\hline 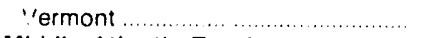 & 7 & • & - & 7 & - & NM \\
\hline Middle Atlantic Total & 1,368 & 1,321 & 1,420 & 2,689 & 2,870 & -6.3 \\
\hline 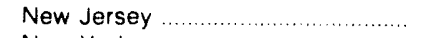 & 52 & 88 & 38 & 140 & 123 & 13.8 \\
\hline New York & 465 & 364 & 413 & 829 & 813 & 2.0 \\
\hline Pennsylvania & 851 & 869 & 970 & 1,719 & 1.933 & -11.1 \\
\hline East North Central Total ..................... & 4,426 & 4,349 & 4,056 & 8,776 & 8,117 & 8.1 \\
\hline 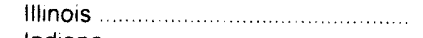 & 1,019 & 1,229 & 649 & 2,249 & 1.538 & 46.2 \\
\hline Indiana & 1.059 & 980 & 905 & 2,040 & 2,009 & 1.5 \\
\hline Michigan & 703 & 704 & 1,071 & 1,407 & 1,631 & -13.7 \\
\hline Ohio & 1.215 & 1.127 & 1,021 & 2,342 & 2,266 & 3.3 \\
\hline Wisconsin & 430 & 308 & 409 & 738 & 672 & 9.8 \\
\hline West North Central Total ................. & 2,369 & 2,158 & 2,374 & 4,528 & 4,581 & -1.2 \\
\hline lowa & 359 & 99 & 395 & 459 & 568 & -19.2 \\
\hline Kansas & 28 & 13 & 28 & 41 & 48 & -14.0 \\
\hline Minnesota & 57 & 161 & 292 & 219 & 521 & -58.0 \\
\hline Missouri & 257 & 276 & 324 & 533 & 630 & -15.4 \\
\hline 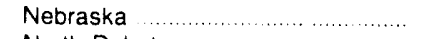 & 24 & 41 & 19 & 65 & 59 & 10.0 \\
\hline North Dakota & 1,581 & 1,528 & 1,246 & 3,109 & 2,645 & 17.5 \\
\hline South Dakota & 64 & 39 & 69 & 103 & 110 & -6.6 \\
\hline South Atlantic Total & 3,339 & 4,100 & 3,911 & 7,439 & 8,010 & -.1 \\
\hline Delaware $\ldots \ldots \ldots \ldots \ldots \ldots \ldots \ldots \ldots$ & 49 & 42 & 43 & 91 & 76 & 20.2 \\
\hline Florida & 175 & 193 & 222 & 367 & 516 & -28.8 \\
\hline Georgia & 447 & 567 & 737 & 1.014 & 1,217 & -16.7 \\
\hline Maryland & 297 & 317 & 192 & 614 & 376 & 63.4 \\
\hline 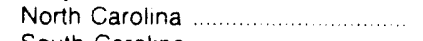 & 572 & 665 & 763 & 1,238 & 1,663 & -25.6 \\
\hline South Carolina & 559 & 499 & 513 & 1.058 & 1,178 & -10.2 \\
\hline Virgunia $\ldots \ldots \ldots \ldots$ & 717 & 1,113 & 899 & 1,830 & 1.779 & 2.9 \\
\hline West Virginia & 524 & 703 & 542 & 1,226 & 1.206 & 1.7 \\
\hline East South Central Total & 1,977 & 1,989 & 1,901 & 3,966 & 4,076 & -2.7 \\
\hline Alabama & 619 & 569 & 616 & 1.188 & 1,267 & -6.3 \\
\hline Kentucky & 391 & 539 & 488 & 931 & 1,100 & -15.4 \\
\hline 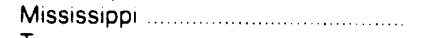 & 29 & 42 & 40 & 72 & 80 & -10.4 \\
\hline Tennessee & 938 & 838 & 758 & 1,776 & 1,628 & 9.0 \\
\hline West South Central Total ................... & 785 & 1,320 & 1,388 & 2,104 & 3,195 & -34.1 \\
\hline Arkansas & 55 & 54 & 58 & 110 & 111 & -1.7 \\
\hline Loulsiana & 120 & 136 & 167 & 256 & 338 & -24.1 \\
\hline Oklahoma & 166 & 149 & 147 & 315 & 263 & 199 \\
\hline Texas & 443 & 980 & 1.017 & 1,423 & 2,483 & -42.7 \\
\hline Mountain Total & 1,112 & 1,228 & 1,083 & 2,341 & 2,155 & 8.6 \\
\hline Arizona & 180 & 189 & 158 & 369 & 339 & 8.7 \\
\hline 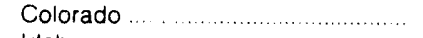 & 147 & 182 & 140 & 329 & 319 & 3.1 \\
\hline Idaho & 120 & 123 & 128 & 243 & 166 & 46.9 \\
\hline 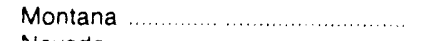 & 61 & 68 & 40 & 130 & 94 & 37.4 \\
\hline Nevada & 57 & 56 & 51 & 113 & 82 & 38.4 \\
\hline New Mexico & 10 & 12 & 10 & 22 & 21 & 5.7 \\
\hline Utah & 134 & 146 & 151 & 279 & 304 & -8.1 \\
\hline 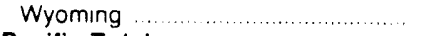 & 403 & 453 & 404 & 856 & 830 & $3 . i$ \\
\hline 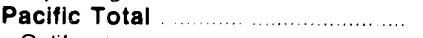 & 772 & 714 & 775 & 1,486 & 1,533 & -3.0 \\
\hline California & 730 & 657 & 735 & 1,388 & 1.424 & -2.5 \\
\hline Oregon & - & 18 & - & 18 & • & NM \\
\hline Washington $\ldots . . \ldots \ldots \ldots \ldots \ldots \ldots \ldots \ldots \ldots \ldots$ & 42 & 39 & 40 & 81 & 109 & -25.7 \\
\hline U.S. Total & 16,177 & 17,197 & 16,940 & 33,374 & 34,647 & -3.7 \\
\hline
\end{tabular}

* Quantity is less than 500 short tons or percent is less than 05

NM Not meaningful as value is greater than or equal to 500 .

Note: Total may not equal sum of components because of independent rounding.

Source: Energy Information Administration, Form EIA-6, "Coal Distribution Report." 


\section{Table 15. Domestic Distribution of U.S. Coal to the Residential and Commercial Sector by Origin \\ (Thousand Short Tons)}

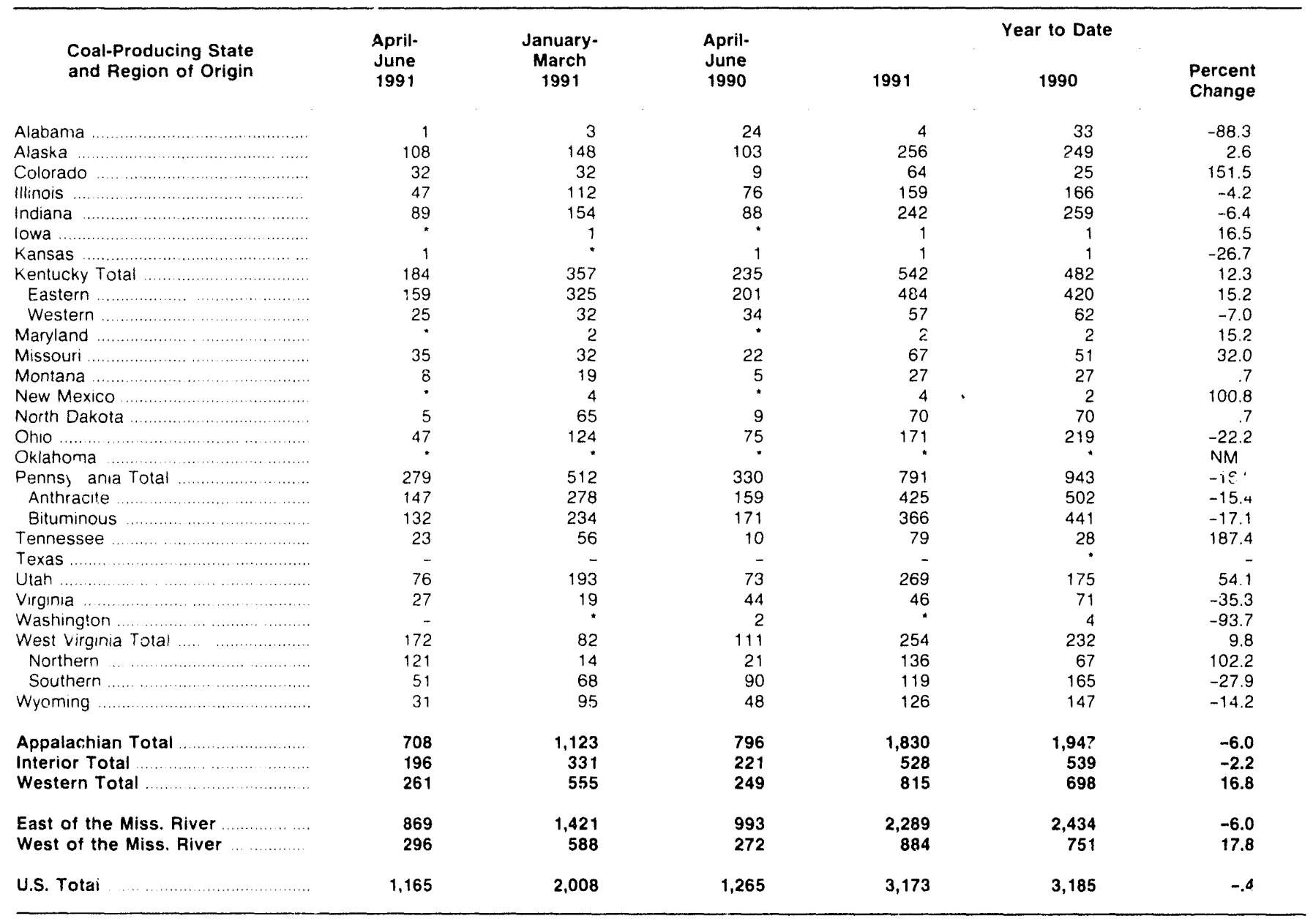

- Quantity is less than 500 short tons or percerit is less than 05 .

NM Not meaningitul as value is greater than or equal to 500 .

Note: Total may not equal sum of components because of independent rounding.

Source: Energy information Administration, Form ElA.6, "Coal Distribution Report." 


\section{Table 16. Domestic Distribution of U.S. Coal to the Residential and Commercial Sector by Destination (Thousand Short Tons)}

\begin{tabular}{|c|c|c|c|c|c|c|}
\hline \multirow{2}{*}{$\begin{array}{l}\text { Census Division and } \\
\text { State of Jestination }\end{array}$} & \multirow{2}{*}{$\begin{array}{l}\text { April- } \\
\text { June } \\
1991\end{array}$} & \multirow{2}{*}{$\begin{array}{c}\text { January- } \\
\text { March } \\
1991\end{array}$} & \multirow{2}{*}{$\begin{array}{l}\text { April- } \\
\text { June } \\
1990\end{array}$} & \multicolumn{3}{|c|}{ Year to Date } \\
\hline & & & & 1991 & 1990 & $\begin{array}{l}\text { Percent } \\
\text { Change }\end{array}$ \\
\hline 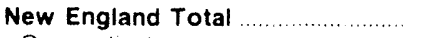 & 9 & 13 & 22 & 23 & 38 & -39.8 \\
\hline 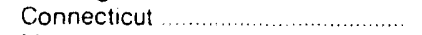 & 1 & 3 & 1 & 3 & 3 & 20 \\
\hline Maine & 1 & 1 & 6 & 3 & 7 & -65.4 \\
\hline Massachusetts & 3 & 5 & 13 & 8 & 20 & -60.4 \\
\hline New Hampshire ............................. & 3 & 3 & 2 & 6 & 4 & 67.0 \\
\hline Rhode Island & - & 1 & $\cdot$ & 1 & 1 & -411 \\
\hline 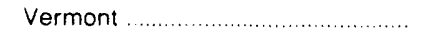 & 1 & 1 & 1 & 2 & 2 & -.3 \\
\hline Middle Atlantic Total ......................... & 270 & 537 & 295 & 807 & 908 & -11.2 \\
\hline New Jersey & 1 & 3 & 2 & 4 & 4 & 6 \\
\hline 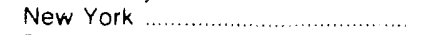 & 46 & 82 & 57 & 128 & 124 & 2.8 \\
\hline 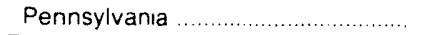 & 223 & 452 & 235 & 675 & 780 & -135 \\
\hline East North Central Total ................ & 227 & 447 & 329 & 674 & 788 & -14.5 \\
\hline 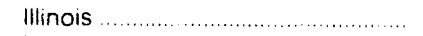 & 21 & 80 & 44 & 102 & 108 & -60 \\
\hline 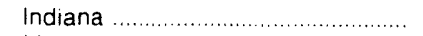 & 95 & 150 & 97 & 245 & 286 & -145 \\
\hline 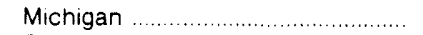 & 54 & 38 & 61 & 92 & 114 & -190 \\
\hline 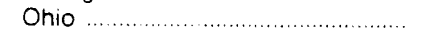 & 54 & 172 & 126 & 226 & 278 & -18.8 \\
\hline 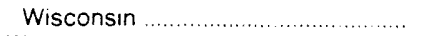 & 3 & 6 & $\cdot$ & 9 & 1 & NM \\
\hline West North Central Total ............. & 180 & 248 & 184 & 428 & 408 & 4.9 \\
\hline lowa & 88 & 64 & 81 & 152 & 121 & 251 \\
\hline 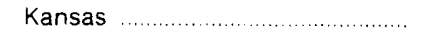 & $\cdot$ & * & $\cdot$ & * & $\cdot$ & NM \\
\hline 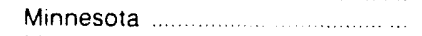 & 17 & 41 & 33 & 58 & 84 & -30.5 \\
\hline 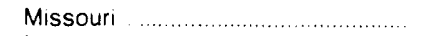 & 59 & 76 & 60 & 136 & 132 & 29 \\
\hline 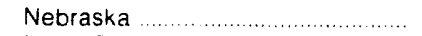 & 7 & $\cdot$ & 1 & 7 & 1 & $N M$ \\
\hline 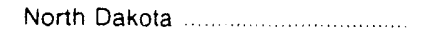 & 9 & 65 & 9 & 74 & 70 & 65 \\
\hline South Dakota ........... & . & 2 & $\cdot$ & 2 & 1 & 55.5 \\
\hline 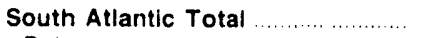 & 184 & 137 & 157 & 321 & 315 & 1.9 \\
\hline 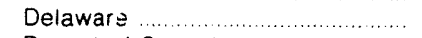 & 4 & 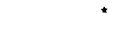 & 1 & 4 & 11 & -59.3 \\
\hline 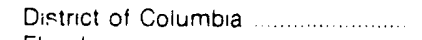 & 13 & 29 & 15 & 41 & 46 & -9.6 \\
\hline Florida $\ldots \ldots \ldots \ldots \ldots \ldots$ & $\cdot$ & - & $\cdot$ & $\cdot$ & $\cdot$ & NM \\
\hline 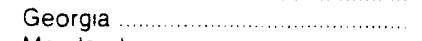 & 2 & 3 & 4 & 4 & 6 & -229 \\
\hline Maryland & 9 & 4 & 13 & 13 & 26 & -52.0 \\
\hline 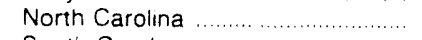 & 18 & 29 & 29 & 47 & 49 & -5.1 \\
\hline 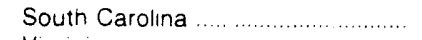 & 1 & 15 & $*$ & 16 & $\cdot$ & NM \\
\hline 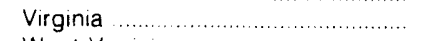 & 26 & 38 & 67 & 64 & 111 & -429 \\
\hline West Virginia & 113 & 20 & 28 & 132 & 66 & 100.3 \\
\hline East South Central Total ............... & 65 & 175 & 67 & 240 & 175 & 37.0 \\
\hline Alabama & 1 & 10 & 28 & 10 & 38 & -725 \\
\hline 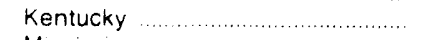 & 14 & 88 & 18 & 102 & 53 & 93.5 \\
\hline 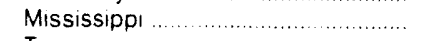 & - & - & $\cdot$ & - & ? & - \\
\hline 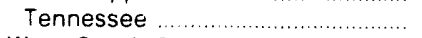 & 50 & 77 & 21 & 128 & 8.4 & 51.0 \\
\hline West South Central Total ................. & 2 & 5 & 6 & 6 & 9 & -30.0 \\
\hline 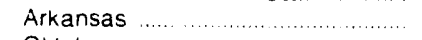 & $\cdot$ & $\cdot$ & - & $\cdot$ & - & - \\
\hline Oklahoma & $\cdot$ & $\cdot$ & * & $\cdot$ & $\cdot$ & NM \\
\hline 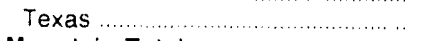 & 2 & 4 & 6 & 6 & 9 & -312 \\
\hline 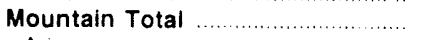 & 96 & 219 & 96 & 314 & 272 & 15.8 \\
\hline 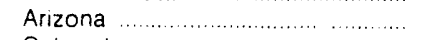 & $\cdot$ & $\cdot$ & . & $?$ & . & NM \\
\hline 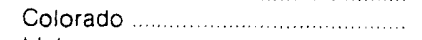 & 12 & 17 & 8 & 28 & 25 & 119 \\
\hline 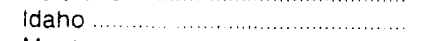 & 8 & 27 & 12 & 36 & 33 & 8.0 \\
\hline 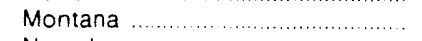 & 4 & 17 & 7 & 21 & 31 & -31.8 \\
\hline 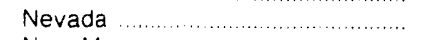 & $\cdot$ & 1 & $\cdot$ & 1 & 1 & 63 \\
\hline 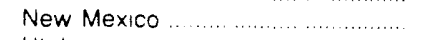 & - & 4 & $\cdot$ & 4 & 2 & 988 \\
\hline 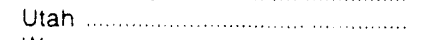 & 58 & 104 & 53 & 161 & 122 & 326 \\
\hline 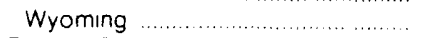 & 14 & 49 & 14 & 63 & 58 & 9.5 \\
\hline 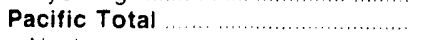 & 132 & 228 & 110 & 360 & 272 & 32.2 \\
\hline 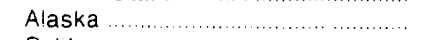 & 108 & 148 & 103 & 256 & 249 & 2.6 \\
\hline 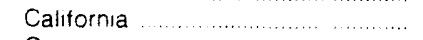 & $\cdot$ & 43 & $\cdot$ & 43 & $\cdot$ & NM \\
\hline 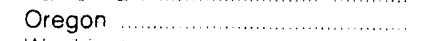 & $\cdot$ & 1 & - & 1 & 1 & 16.0 \\
\hline 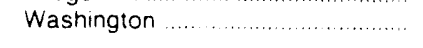 & 24 & 37 & 7 & 60 & 22 & 1725 \\
\hline$\ldots \ldots \ldots \ldots \ldots \ldots \ldots$ & 1,165 & 2,008 & 1,265 & 3,173 & 3,185 & -.4 \\
\hline
\end{tabular}

- Ouantity is less than 500 short tons or percent is less than 05

NM Not meaningful as value is greater than or equal to 500 .

Note. Total may not equal sum of components because of independent rounding.

Source: Energy Information Administration. Form EłA-6. "Coal Distribution Report." 


\section{Methods of Transportation}

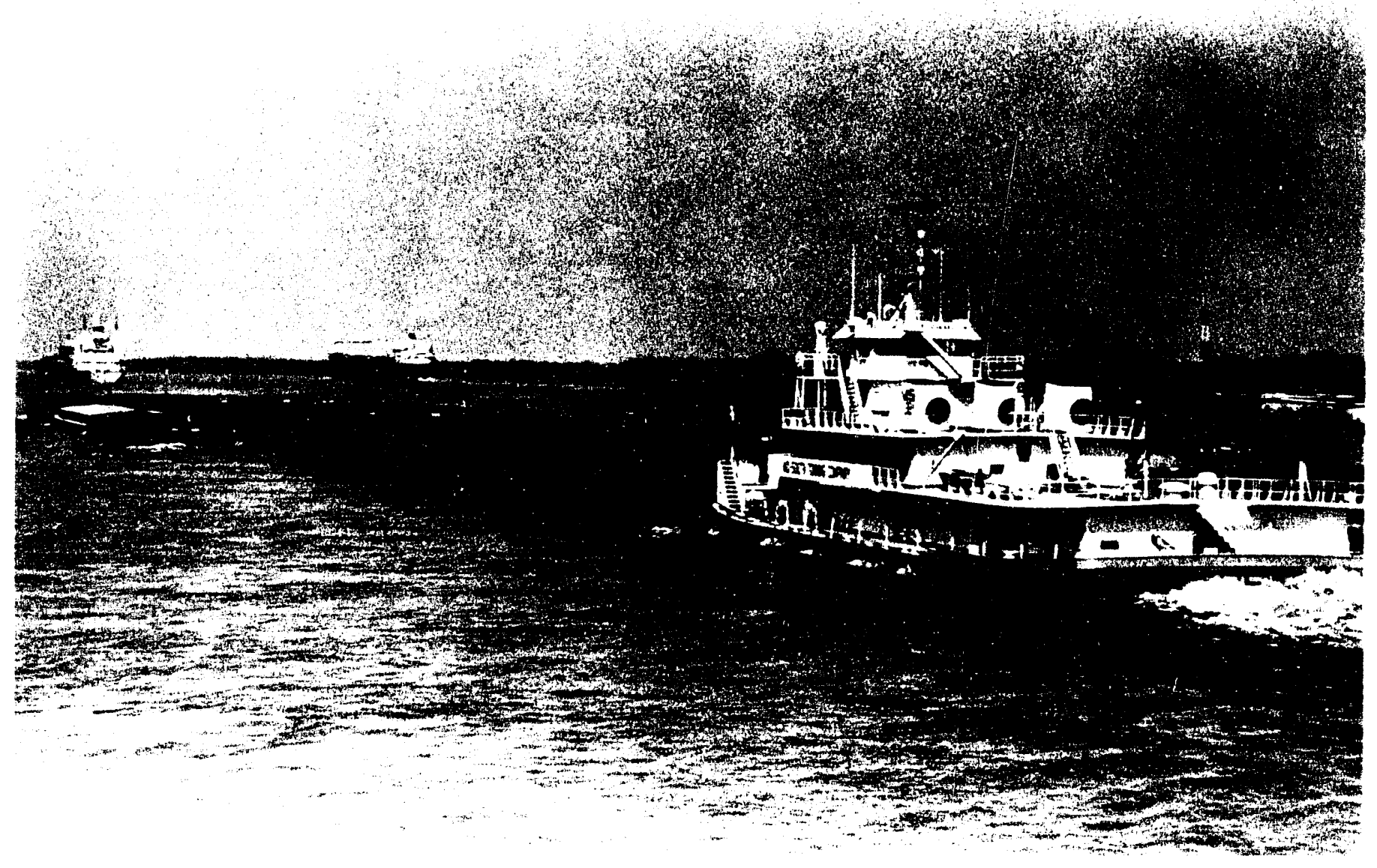

Barges on the Mississippi river carrying coal from upriver terminals near midwestern mines io iranisfer factilities downitiver. River transportation accounted for 15 percent of all domestic shipments in 1990. 
Figure 4. Domestic Distribution of U.S. Coal by Method of Transportation, January-June 1991

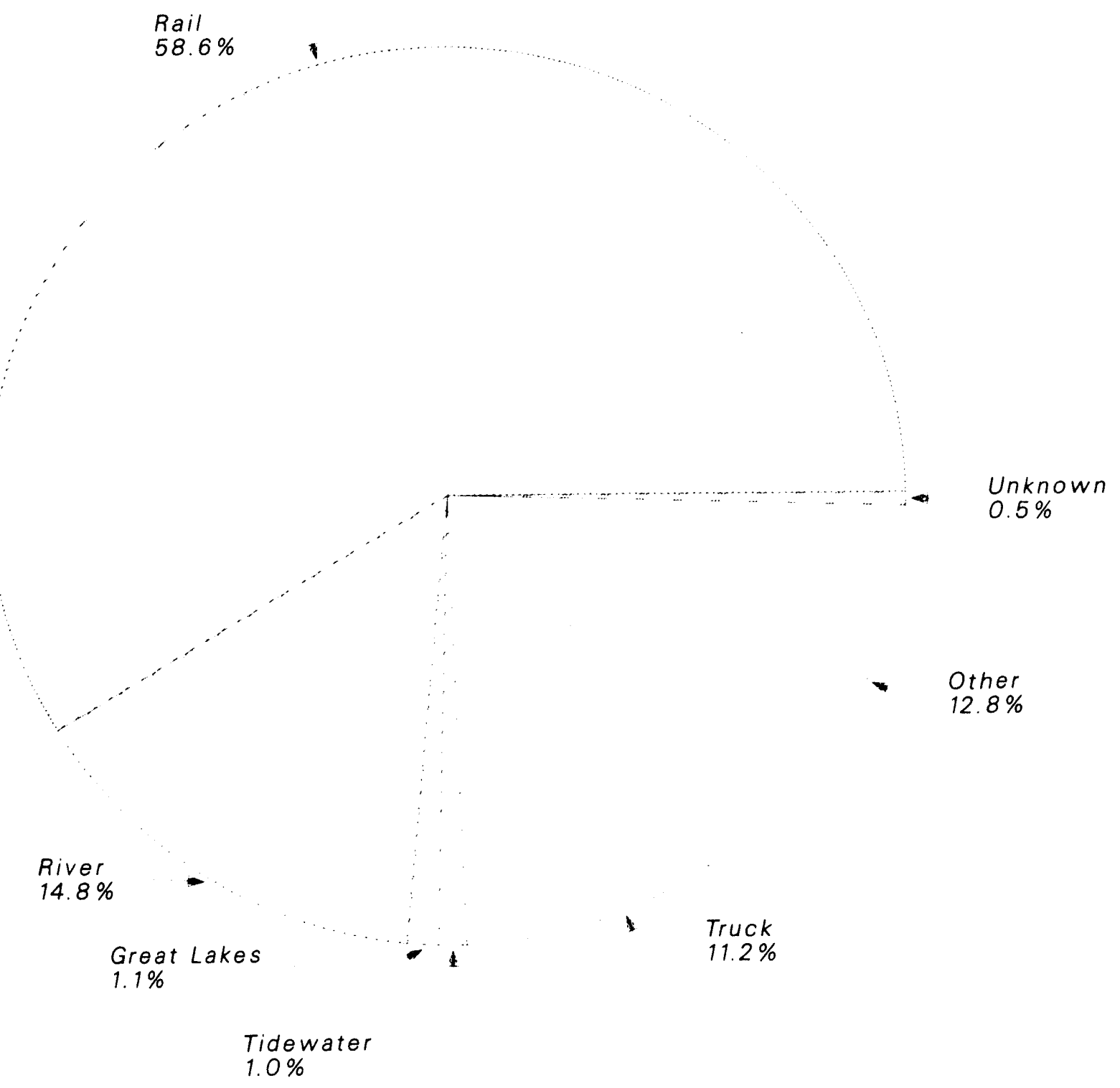

Note: Other methods of transportation include tramivay, conveyor, and slurry pipeline. Source: Energy Information Administration. Form ElA-6. "Coal Distribution Report." 
Table 17. Domestic Distribution of U.S. Coal by Origin and Method of Transportation, January-June 1991

(Thousand Short Tons)

\begin{tabular}{|c|c|c|c|c|c|c|c|c|c|}
\hline \multirow{2}{*}{$\begin{array}{l}\text { Coal-Producing State } \\
\text { and Region of Origin }\end{array}$} & \multirow{2}{*}{ Railroad } & \multicolumn{4}{|c|}{ Water } & \multirow{2}{*}{ Truck } & \multirow{2}{*}{$\begin{array}{l}\text { Tramway, } \\
\text { Conveyor, } \\
\text { and Slurry } \\
\text { Plpeline }\end{array}$} & \multirow{2}{*}{ Unknown } & \multirow{2}{*}{ Total } \\
\hline & & River & $\begin{array}{l}\text { Great } \\
\text { Lakes }\end{array}$ & Tidewater & Total & & & & \\
\hline Alabama & 3,436 & 1.115 & - & - & 1,115 & 4.489 & 855 & 354 & 10.249 \\
\hline 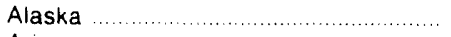 & 328 & - & - & - & - & 84 & - & - & 412 \\
\hline Arizona $\ldots \ldots \ldots \ldots$ & 3,787 & - & - & - & - & - & 2.575 & - & 6.362 \\
\hline 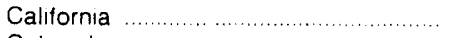 & - & - & - & - & - & 8 & - & - & 8 \\
\hline 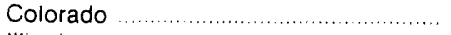 & 6.422 & - & - & - & - & 2.345 & - & 2 & 8.708 \\
\hline Illinois ..... & 14,926 & 8,888 & 10 & - & 8.898 & 3.111 & 1,237 & 21 & 28,193 \\
\hline 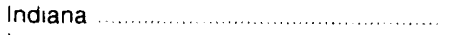 & 8.172 & 3.099 & 146 & - & 3.245 & 2.913 & 352 & 135 & 14,818 \\
\hline$\ldots \ldots \ldots \ldots \ldots \ldots \ldots \ldots \ldots \ldots \ldots \ldots \ldots$ & - & - & - & - & - & 175 & - & - & 175 \\
\hline 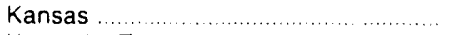 & 27 & - & - & - & - & 230 & - & - & 258 \\
\hline 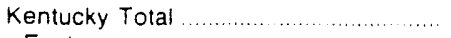 & 41,232 & 18,743 & 715 & 1.453 & 20,911 & 4.796 & 1.275 & 768 & 68.981 \\
\hline 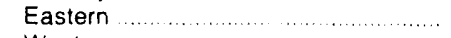 & 36,442 & 6.917 & 642 & 1.306 & 8,865 & 2.994 & - & 715 & 49.017 \\
\hline 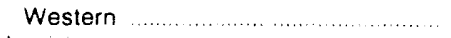 & 4.789 & 11,826 & 73 & 147 & 12,046 & 1.802 & 1.275 & 52 & 19,964 \\
\hline 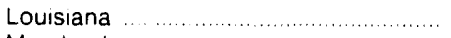 & - & - & - & - & - & 177 & 1.111 & - & 1.287 \\
\hline 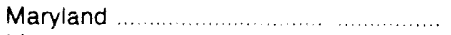 & 1.222 & - & - & - & - & 416 & - & 18 & 1.655 \\
\hline 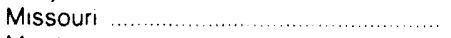 & 10 & - & - & & - & 164 & 821 & 2 & 997 \\
\hline 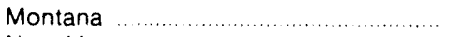 & 10,293 & 102 & 2.918 & - & 3.020 & 191 & 4.397 & 2 & 17.903 \\
\hline New Mexico & 4,829 & - & - & - & - & 3 & 5.355 & $\cdot$ & 10.188 \\
\hline 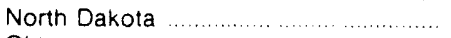 & 2,203 & - & - & - & - & 1.895 & 10,506 & 1 & 14.604 \\
\hline 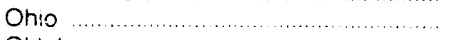 & 1.197 & 4,266 & 40 & 167 & 4,474 & 5.101 & 4.551 & 62 & 15,386 \\
\hline 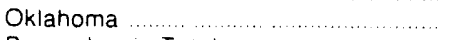 & 90 & - & - & - & - & 700 & - & 3 & 793 \\
\hline 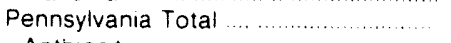 & 13.209 & 3,194 & 322 & 100 & 3.616 & 6.986 & 4,939 & 198 & 28,948 \\
\hline Anthracite $\ldots \ldots \ldots \ldots \ldots \ldots$ & 84 & - & - & - & - & 926 & 194 & 23 & 1.227 \\
\hline 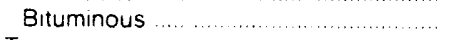 & 13.125 & 3,194 & 322 & 100 & 3.616 & 6,060 & 4.745 & 175 & 27.721 \\
\hline 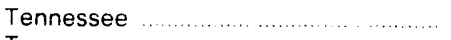 & 1,538 & 539 & 18 & - & 558 & 248 & - & 44 & 2,388 \\
\hline 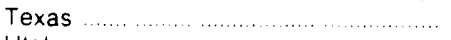 & 10.714 & - & - & - & - & 6.521 & 8.623 & - & 25.858 \\
\hline 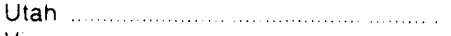 & 5.490 & - & - & - & - & 3,028 & 1.194 & 3 & 9.715 \\
\hline 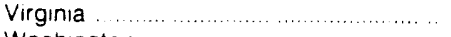 & 9,905 & 1,054 & - & 733 & 1,787 & 40 & 480 & 40 & 12.252 \\
\hline 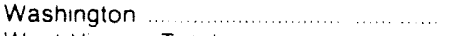 & 2.193 & - & - & - & - & $\cdot$ & - & - & 2.193 \\
\hline 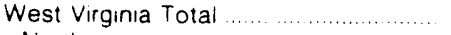 & 30,547 & 16.043 & 334 & 1,775 & 18,152 & 3.583 & 2.282 & 430 & 54,994 \\
\hline 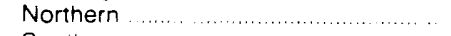 & 8.753 & 7,865 & 171 & 1.044 & 9.080 & 2.231 & 2.282 & 248 & 22,594 \\
\hline Southern $\ldots \ldots \ldots \ldots \ldots \ldots \ldots \ldots$ & 21.794 & 8.178 & 163 & 731 & 9.072 & 1.353 & - & 182 & 32,400 \\
\hline 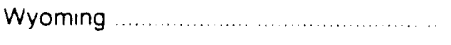 & 81.829 & 6.796 & 233 & - & 7.029 & 1.408 & 4.788 & 20 & 95.073 \\
\hline $\begin{array}{l}\text { Appalachian Total } \\
\text { Interior Total }\end{array}$ & $\begin{array}{l}97,496 \\
38,728\end{array}$ & $\begin{array}{l}33,129 \\
23,814\end{array}$ & $\begin{array}{r}1,356 \\
229\end{array}$ & $\begin{array}{r}4,080 \\
147\end{array}$ & $\begin{array}{l}38,565 \\
24,190\end{array}$ & $\begin{array}{l}23,858 \\
15,793\end{array}$ & $\begin{array}{l}13,107 \\
13.419\end{array}$ & $\begin{array}{r}1.862 \\
213\end{array}$ & $\begin{array}{r}174,889 \\
92,342\end{array}$ \\
\hline 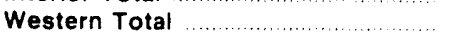 & 117,372 & 6,898 & 3,151 & - & 10,049 & 8,962 & 28,816 & 28 & $\begin{array}{r}92,042 \\
165,226\end{array}$ \\
\hline $\begin{array}{l}\text { East of the Miss. River } \\
\text { West of the Miss. River }\end{array}$ & $\begin{array}{l}125,383 \\
128,2 \div 3\end{array}$ & $\begin{array}{r}56,943 \\
6,898\end{array}$ & $\begin{array}{l}1,585 \\
3,151\end{array}$ & $\begin{array}{r}4,228 \\
-\end{array}$ & $\begin{array}{l}62,755 \\
10,049\end{array}$ & $\begin{array}{l}31,684 \\
16,929\end{array}$ & $\begin{array}{l}15,971 \\
39,371\end{array}$ & $\begin{array}{r}2,071 \\
32\end{array}$ & $\begin{array}{l}237,864 \\
194,593\end{array}$ \\
\hline U.S. Total & 253,596 & 63,841 & 4,736 & 4,228 & 72,804 & 48,613 & 55,342 & 2,103 & 432,457 \\
\hline
\end{tabular}

- Quanuty is less than 500 short tons or percent is less than 05

Note. Total may not equal sum of components because of inc ependent rounding

Source: Energy Information Administration. Form EIA-6. "Coal Distribution Report." 


\section{Table 18. Foreign Distribution of U.S. Coal by Origin and Method of Transportation, January-June 1991}

(Thousand Short Tons)

\begin{tabular}{|c|c|c|c|c|c|c|c|c|}
\hline \multirow{3}{*}{$\begin{array}{l}\text { Coal-Producing State } \\
\text { and Region of Origin }\end{array}$} & \multicolumn{6}{|c|}{ Canada } & \multirow{3}{*}{ Overseas' } & \multirow{3}{*}{ Total } \\
\hline & \multirow[b]{2}{*}{ Railroad } & \multicolumn{2}{|r|}{ Water } & & \multirow[b]{2}{*}{ Truck } & \multirow[b]{2}{*}{ Total } & & \\
\hline & & $\begin{array}{l}\text { Great } \\
\text { Lakes }\end{array}$ & Tidewater & Total & & & & \\
\hline Alabama & - & - & - & - & - & - & 3,992 & 3,992 \\
\hline 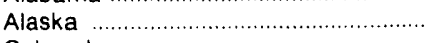 & - & - & - & - & - & - & 340 & 340 \\
\hline 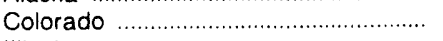 & - & - & - & - & - & - & 332 & 332 \\
\hline 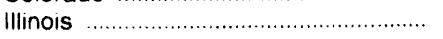 & - & - & - & - & - & - & 400 & 400 \\
\hline 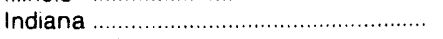 & - & 3 & - & 3 & - & 3 & 59 & 62 \\
\hline 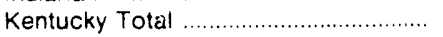 & * & 223 & 64 & 287 & 1 & 288 & 6,605 & 6,893 \\
\hline 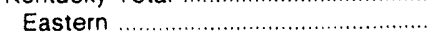 & $\cdot$ & 223 & 64 & 287 & 1 & 288 & 6.442 & 6.730 \\
\hline 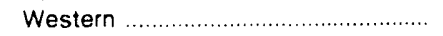 & - & - & - & - & - & - & 163 & 163 \\
\hline 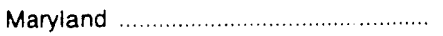 & - & - & - & - & - & - & 28 & 28 \\
\hline 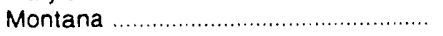 & - & - & - & - & - & - & 148 & 148 \\
\hline Onio & - & - & - & - & - & - & 14 & 14 \\
\hline Pennsylvania Total ................................ & 57 & 489 & 70 & 560 & 10 & 627 & 1,857 & 2,484 \\
\hline 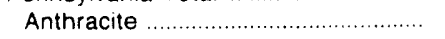 & 57 & $*$ & 70 & 71 & 4 & 132 & 14 & 145 \\
\hline 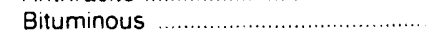 & - & 489 & - & 489 & 6 & 495 & 1,844 & 2,339 \\
\hline 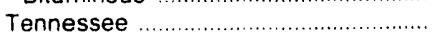 & - & 4 & - & 4 & - & 4 & 2 & 6 \\
\hline 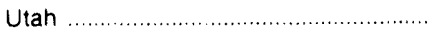 & - & - & - & - & - & - & 819 & 819 \\
\hline 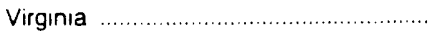 & - & 572 & - & 572 & - & 572 & 8,713 & 9,285 \\
\hline 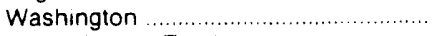 & - & - & - & - & - & - & 112 & 112 \\
\hline West Virginia Total ................................ & 220 & 2,467 & 235 & 2,703 & - & 2,923 & 21,926 & 24,849 \\
\hline 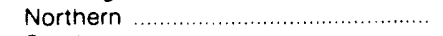 & - & 770 & - & 770 & - & 770 & 2,272 & 3.042 \\
\hline 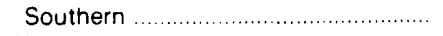 & 220 & 1,698 & 235 & 1,933 & - & 2,153 & 19,654 & 21,807 \\
\hline Wyoming & - & - & - & - & - & - & 461 & 461 \\
\hline Appalachian Total $\ldots \ldots \ldots \ldots \ldots \ldots \ldots \ldots$ & 277 & 3,756 & 369 & 4,125 & 11 & 4,413 & 42,975 & 47,388 \\
\hline 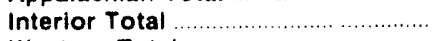 & - & 3 & - & 3 & - & 3 & 622 & 625 \\
\hline 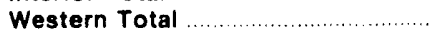 & - & - & - & - & - & - & 2,212 & 2,212 \\
\hline East of the Miss. River & 277 & 3,759 & 369 & 4,129 & 11 & 4,416 & 43,597 & 48,013 \\
\hline West cf the Miss. River ................... & - & - & - & - & - & - & 2,212 & 2,212 \\
\hline U.S. Total & 277 & 3,759 & 369 & 4,129 & 11 & 4,416 & 45,809 & 50,226 \\
\hline
\end{tabular}

1 Also includes Mexico

Quantity is less than 500 short tons or percent is less than 05

Note: Total may not equal sum of components because of independent rounding.

Source: Energy Information Administration, Form EIA.6, "Coal Distribution Report." 
Table 19. Domestic Distribution of U.S. Coal by Destination and Method of Transportation, January-June 1991

(Thousand Short Tons)

\begin{tabular}{|c|c|c|c|c|c|c|c|c|}
\hline \multirow{2}{*}{$\begin{array}{l}\text { Census Division and } \\
\text { State of Destination }\end{array}$} & \multirow{2}{*}{ Railroad } & \multicolumn{4}{|c|}{ Water } & \multirow{2}{*}{\multicolumn{2}{|c|}{$\begin{array}{l}\text { Tramway, } \\
\text { Conveyor, } \\
\text { and Slurry } \\
\text { Pipeline }\end{array}$}} & \multirow{2}{*}{ Total } \\
\hline & & River & $\begin{array}{l}\text { Great } \\
\text { Lakes }\end{array}$ & Tidewater & Total & & & \\
\hline New England Total & 1,129 & - & - & 1.657 & 1,657 & 44 & - & 2,830 \\
\hline Connecticut & 191 & - & - & 332 & 332 & 3 & - & 527 \\
\hline ....................... & 11 & - & $\rightarrow$ & - & - & 2 & - & 12 \\
\hline Massachusetts & 444 & - & - & 1.096 & 1.096 & 32 & - & 1,572 \\
\hline 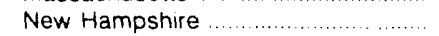 & 476 & - & - & 229 & 229 & 5 & - & 710 \\
\hline 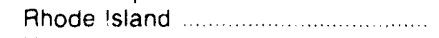 & $\cdot$ & - & - & - & - & 1 & - & 1 \\
\hline Vermont & 7 & - & - & - & - & 2 & - & 9 \\
\hline Middle Atlantic Total & 13,794 & 8,023 & 29 & 424 & 8.475 & 6,736 & 4,939 & 33,973 \\
\hline 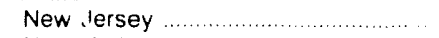 & 536 & 41 & - & 391 & 431 & 8 & - & 975 \\
\hline New York ..... & 5,646 & 306 & 7 & - & 312 & 534 & - & 6.492 \\
\hline Pennsylvania ........................ & 7,612 & 7.676 & 22 & 34 & 7.732 & 6.194 & 4.939 & 126,505 \\
\hline East North Central Total & 53,828 & 23,093 & 4,663 & - & 27,756 & 11,663 & 6,057 & 199,335 \\
\hline 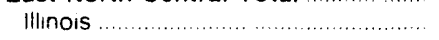 & 10,153 & 2.609 & 211 & - & 2.819 & 2.853 & 1.237 & 117.077 \\
\hline 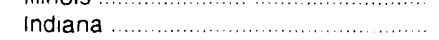 & 16.778 & 6,947 & - & - & 6.947 & 2.887 & 352 & 26,976 \\
\hline Michigan & 10.935 & 8 & 3,883 & - & 3,891 & 247 & - & 15.072 \\
\hline (1) & 6,590 & 12.750 & 210 & - & 12.960 & 5.624 & 4.468 & 29,647 \\
\hline Wisconsin & 9,372 & 779 & 360 & - & 1,138 & 53 & - & 10,564 \\
\hline West North Central Total & 37,862 & 3,753 & 44 & - & 3,796 & 3,112 & 11,327 & 56,097 \\
\hline lowa & 7.280 & 1.534 & - & - & 1.534 & 183 & - & 8.996 \\
\hline 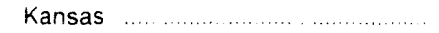 & 6.849 & 8 & - & - & 8 & 91 & - & 6.949 \\
\hline Minnesota & 8,699 & 48 & 39 & - & 87 & 31 & - & 8.817 \\
\hline 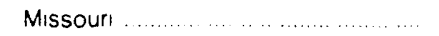 & 8.409 & 2.162 & - & - & 2.162 & 685 & 821 & 12.077 \\
\hline Nebraska & 4.278 & - & - & - & - & - & - & 4,278 \\
\hline North Dakoia. & 1.069 & - & - & - & - & 1.895 & 10.506 & 113,470 \\
\hline South Dakota & 1.278 & - & 5 & - & 5 & 227 & - & 1.510 \\
\hline South Atlantic Total & 51,201 & 11,331 & - & 2,143 & 13,474 & 3,855 & 2,845 & 171,464 \\
\hline 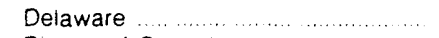 & 1.102 & - & - & - & - & 3 & - & 1,105 \\
\hline 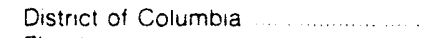 & 35 & - & - & - & - & 6 & - & 41 \\
\hline 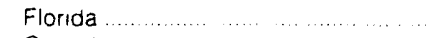 & 5,489 & 4.537 & - & 1.354 & 5.891 & 4 & - & 11.384 \\
\hline 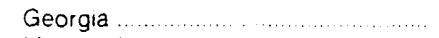 & 10.732 & 1.829 & - & - & 1.829 & 86 & - & 12,647 \\
\hline 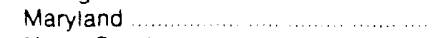 & 4,522 & 78 & - & 567 & 645 & 244 & - & 15,422 \\
\hline 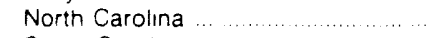 & 10.295 & - & - & - & - & 200 & - & 10,495 \\
\hline 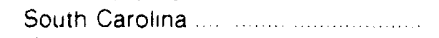 & 5.280 & - & - & - & - & 90 & - & 5,371 \\
\hline 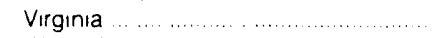 & 5.956 & 1 & - & 222 & 223 & 356 & 480 & 17,031 \\
\hline West Virginia & 7,789 & 4.885 & - & - & 4.885 & 2.866 & 2.366 & $1,17,967$ \\
\hline East South Central Total & 17,990 & 14,917 & - & - & 14,917 & 9,006 & 2,130 & 144,147 \\
\hline Alabama & 5.195 & 3.165 & - & - & 3.165 & 4.663 & 855 & 113.937 \\
\hline 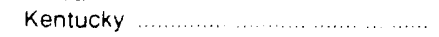 & 5.724 & 4.348 & - & - & 4.348 & 3.757 & 1.275 & 15.148 \\
\hline Mississippi & 1.074 & 810 & - & - & 810 & 11 & - & 1,895 \\
\hline Tennessee & 5,997 & 6.594 & - & - & 6.594 & 575 & - & 113,167 \\
\hline West South Central Total .................. & 45,587 & 2,725 & - & 4 & 2,730 & 7,400 & 9,734 & 65,451 \\
\hline 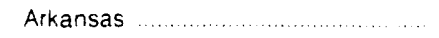 & 6.210 & - & - & - & - & 56 & - & 6,265 \\
\hline 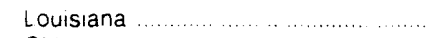 & 2.043 & 2.714 & - & 4 & 2.718 & 177 & 1,111 & 6,048 \\
\hline 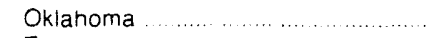 & 7.837 & 9 & - & - & 9 & 646 & - & 18.492 \\
\hline Texas & 29.498 & 3 & - & - & 3 & 6,521 & 8.623 & 44.645 \\
\hline Mountain Total ....... & 27,059 & - & - & - & - & 6,704 & 18,310 & 52,087 \\
\hline Arizona & 8.492 & - & - & - & - & 10 & - & 8,502 \\
\hline 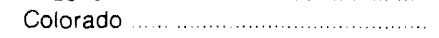 & 5.664 & - & - & - & - & 2.306 & - & 17.970 \\
\hline idaho & 145 & - & - & - & - & 134 & - & 279 \\
\hline 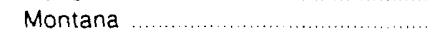 & 551 & - & - & - & - & 184 & 4,397 & 5.132 \\
\hline 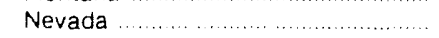 & 1.708 & - & - & - & . & 22 & 2.575 & 4,305 \\
\hline New Mexico & 410 & - & - & - & - & 33 & 5,355 & 15.799 \\
\hline Utah & 3,907 & - & - & - & - & 2,898 & 1.194 & 8,001 \\
\hline Wyoming & 6.183 & - & - & - & - & 1.117 & 4.788 & $1,12,098$ \\
\hline Pacific Total & 5,146 & - & - & - & - & 94 & $4,1.00$ & 5,240 \\
\hline Alaska & 328 & - & - & - & - & 84 & - & 412 \\
\hline Calıfornia & 1.422 & - & - & - & - & 9 & - & 1.431 \\
\hline Oregon & 1.063 & - & - & - & - & 1 & - & 1.063 \\
\hline Washington $\ldots \ldots \ldots$ & 2.333 & - & - & - & $\ldots$ & 1 & - & 2,334 \\
\hline Unknown & - & - & - & - & - & - & - & 11,834 \\
\hline U.S. Total & 253,596 & 63,841 & 4,736 & 4,228 & 72,804 & 48,613 & 55,342 & 1432,457 \\
\hline
\end{tabular}

1 Includes distribution using unknown or withneld methods of transportation

Quantity is less than 500 short tons or percent is less than 05.

Note: Total may not equal sum of components because of independent rounding.

Source Energy information Administration. Form E|A-6, "Coal Distribution Report." 
Table 20. Secondary Methods of Transportation by Origin, January-June 1991 (Thousand Short Tons)

\begin{tabular}{|c|c|c|c|c|c|}
\hline \multirow{2}{*}{$\begin{array}{l}\text { Coal-Producing State } \\
\text { and Region of Origin }\end{array}$} & \multicolumn{5}{|c|}{ How Coal was Delivered to the Tidewater Piers and Coastal Ports } \\
\hline & Rail & Truck & River & Unknown & Total \\
\hline Alabama & 955 & - & 3,037 & . & 3.992 \\
\hline 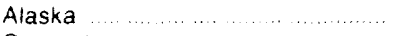 & 340 & - & - & - & 340 \\
\hline 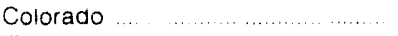 & 332 & - & - & - & 332 \\
\hline - & 215 & - & 185 & - & 400 \\
\hline - & - & - & 21 & 38 & 59 \\
\hline Kentucky Total & 6,705 & 60 & 1.354 & 2 & 8,121 \\
\hline Eastern & 6,649 & 60 & 1,101 & 2 & 7,811 \\
\hline Western & 57 & - & 253 & - & 310 \\
\hline Maryland & 27 & 1 & - & - & 28 \\
\hline Montana & 148 & - & - & - & 148 \\
\hline Onio & 171 & - & 11 & - & 181 \\
\hline Pennsylvania Total & 1,773 & 121 & 134 & - & 2.027 \\
\hline Anthracite & 83 & 1 & - & - & 84 \\
\hline Bituminous & 1,689 & 120 & 134 & - & 1.944 \\
\hline 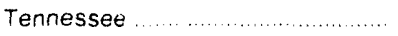 & - & & 2 & - & 2 \\
\hline Utah & 764 & 56 & - & - & 819 \\
\hline ……............. & 9,304 & - & 142 & - & 9.446 \\
\hline Washington. & 112 & - & - & - & 112 \\
\hline West Virginia Total & 20.933 & 412 & 2,570 & 22 & 23.937 \\
\hline Northern & 3,032 & - & 284 & - & 3,316 \\
\hline Southern & 17,901 & 412 & 2.286 & 22 & 20.621 \\
\hline Wyoming & 461 & - & - & - & 461 \\
\hline Appalachian Total & 39,810 & 595 & 6,996 & 23 & 47,425 \\
\hline 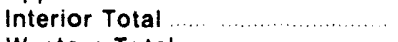 & 272 & - & 459 & 38 & 769 \\
\hline Western Total .......................... & 2,157 & 56 & - & - & 2,212 \\
\hline $\begin{array}{l}\text { East of the Miss. River } \\
\text { West of the Miss. River }\end{array}$ & $\begin{array}{r}40,082 \\
2,157\end{array}$ & $\begin{array}{r}595 \\
56\end{array}$ & $\begin{array}{r}7,455 \\
-\end{array}$ & 61 & $\begin{array}{r}48,194 \\
2,212\end{array}$ \\
\hline \multirow[t]{3}{*}{ U.S. Total } & 42,239 & 651 & 7,455 & 61 & 50,406 \\
\hline & \multicolumn{5}{|c|}{ How Coal was Delivered to the Great Lakes } \\
\hline & Rail & Truck & River & Unknown & Total \\
\hline lilinois & 10 & - & - & - & 10 \\
\hline Indiana & 139 & - & - & 10 & 149 \\
\hline Kentucky Total & 806 & 63 & 27 & 42 & 938 \\
\hline 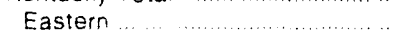 & 745 & 63 & 15 & 42 & 865 \\
\hline Western & 61 & - & 12 & - & 73 \\
\hline Montana & 2.918 & - & - & - & 2,918 \\
\hline Ohio & 34 & $\cdot$ & 6 & - & 40 \\
\hline Pennsyivania Total & 811 & - & - & - & 811 \\
\hline Anthracite & * & - & - & - & \\
\hline Bituminous & 811 & - & - & - & 811 \\
\hline Tennessee & 23 & - & - & - & 23 \\
\hline Virginaa & 572 & - & - & - & 572 \\
\hline West Virginia Total & 2.662 & 133 & 6 & - & 2.801 \\
\hline Northern & 941 & - & - & - & 941 \\
\hline Southern & 1.722 & 133 & 6 & - & 1,860 \\
\hline Wyoming & 233 & - & - & - & 233 \\
\hline Appalachian Total & 4,847 & 196 & 26 & 42 & 5,112 \\
\hline Interior Total & 210 & - & 12 & 10 & 232 \\
\hline 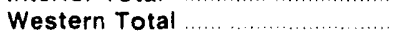 & 3,151 & - & - & - & 3,151 \\
\hline East of the Miss. River & 5,057 & 196 & 39 & 52 & $\begin{array}{l}5,344 \\
3,151\end{array}$ \\
\hline West of the Miss. River & 3,151 & - & - & - & 3,151 \\
\hline U.S. Total & 8,208 & 196 & 39 & 52 & 8,495 \\
\hline
\end{tabular}

See footnotes at end of table. 


\section{Table 20. Secondary Methods of Transportation by Origin, January-June 1991 (Continued) \\ (Thousand Short Tons)}

\begin{tabular}{|c|c|c|c|c|c|}
\hline \multirow{2}{*}{$\begin{array}{l}\text { Coal-Producing } \\
\text { Region of Origin }\end{array}$} & \multicolumn{5}{|c|}{ How Coal was Delivered to the Rivers } \\
\hline & Rail & Truck & Conveyor Belt & Unknown & Total \\
\hline Alabama & 124 & 991 & - & - & 1.115 \\
\hline 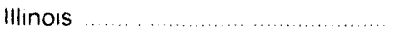 & 7.592 & 969 & 208 & 19 & 8.888 \\
\hline Indiana $\ldots . . . . .$. & 1,648 & 1.440 & - & 12 & 3.099 \\
\hline Kentucky Total & 7.063 & 8.811 & 2.716 & 154 & 18.743 \\
\hline Eastern & 2.757 & 4.013 & - & 148 & 6.917 \\
\hline Western & 4,306 & 4.798 & 2.716 & 6 & 11,826 \\
\hline Montana & 102 & - & - & - & 102 \\
\hline 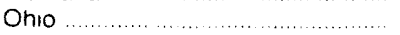 & 638 & 3,557 & 45 & 26 & 4.266 \\
\hline Pennsylvania Total $\ldots . . . . . . . . . . . . . . . . .$. & 1.095 & 1.158 & 919 & 22 & 3.194 \\
\hline Bituminous & 1.095 & 1.158 & 919 & 22 & 3,194 \\
\hline Tennessee .... & 85 & 436 & - & 18 & 539 \\
\hline Virgina & 1.054 & - & - & - & 1.054 \\
\hline West Virginia Total ...................... & 3.261 & 5.543 & 4.031 & 3.208 & 16.043 \\
\hline Northern & 181 & 958 & 3,527 & 3.199 & 7.865 \\
\hline Southern & 3.080 & 4.585 & 504 & 9 & 8.178 \\
\hline 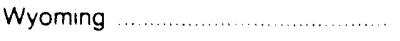 & 6,796 & - & - & - & 6.796 \\
\hline Appalachian Total & 9,014 & 15,697 & 4,995 & 3,423 & 33,129 \\
\hline Interior Total $\ldots$ & 13,646 & 7,207 & 2,925 & 36 & 23,814 \\
\hline Western Total $\ldots \ldots \ldots \ldots$ & 6,898 & - & - & - & 6,898 \\
\hline $\begin{array}{l}\text { East of the Miss. River } \\
\text { West of the Miss. River }\end{array}$ & $\begin{array}{r}22,660 \\
6,898\end{array}$ & $\begin{array}{r}22,904 \\
-\end{array}$ & $\begin{array}{r}7,920 \\
-\end{array}$ & 3,459 & $\begin{array}{r}56,943 \\
6,898\end{array}$ \\
\hline U.S. Total & 29,558 & 22,904 & 7,920 & 3,459 & 63,841 \\
\hline
\end{tabular}

- Quantity is less than 500 short tons or percent is less than 05 .

Note: Total may not equal sum of components because of independent rounding

Source Energy Information Administration. Form EIA.6. "Coal Distribution Report. 
Figure 5. Quarterly Domestic Coal Distribution by Method of Transportation, 1983-1991

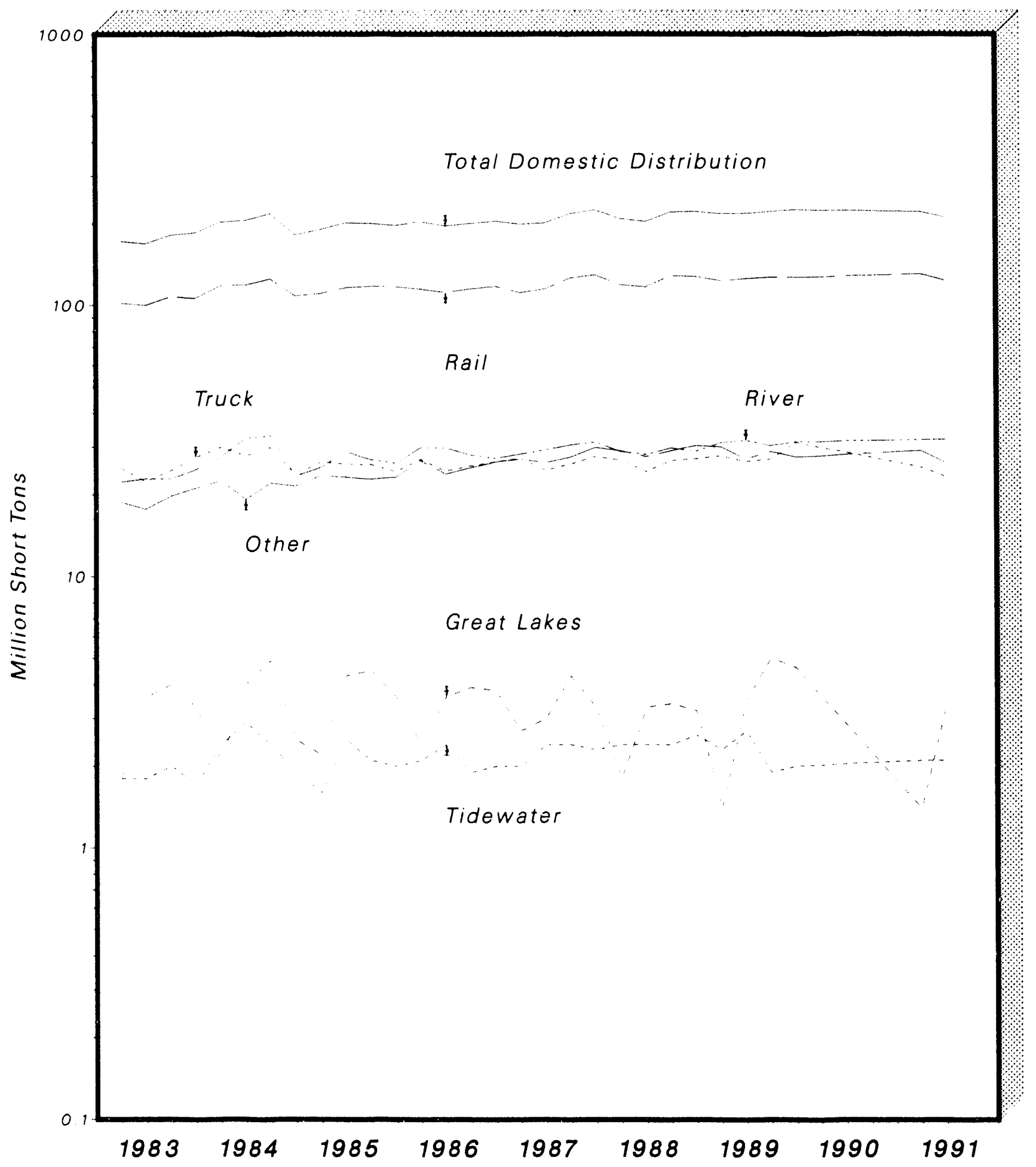

Note: Each increment represents end of quarter data. Other methods of transportation include tramway. conveyor, or slurry pipeline.

Source: Energy Intormation Administration. Form ElA-6, "Coal Distribution Report," 
Table 21. Domestic Distribution of U.S. Coal via Rail by Origin (Thousand Short Tons)

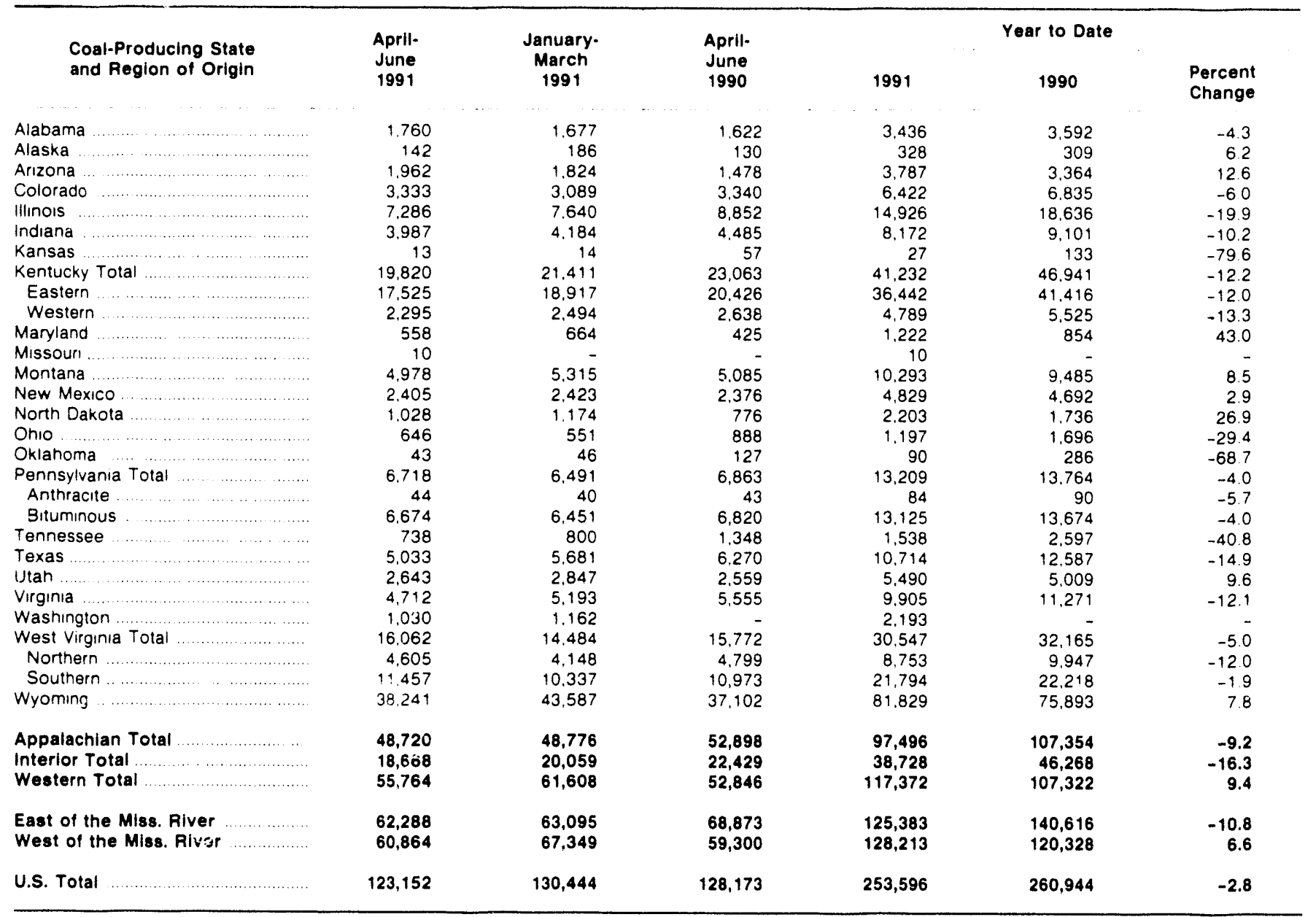

Note: Total may not equal sum of components because of independent rounding.

Source: Energy Information Administration. Form EIA-6. "Coal Distribution Report." 
Table 22. Domestic Distribution of U.S. Coal via Rail by Destination

(Thousand Short Tons)

\begin{tabular}{|c|c|c|c|c|c|c|}
\hline \multirow{2}{*}{$\begin{array}{l}\text { Census Division and } \\
\text { State of Destination }\end{array}$} & \multirow{2}{*}{$\begin{array}{l}\text { April. } \\
\text { June } \\
1991\end{array}$} & \multirow{2}{*}{$\begin{array}{c}\text { January- } \\
\text { March } \\
1991\end{array}$} & \multirow{2}{*}{$\begin{array}{l}\text { April- } \\
\text { June } \\
1990\end{array}$} & \multicolumn{3}{|c|}{ Year to Date } \\
\hline & & & & 1991 & 1990 & $\begin{array}{l}\text { Percent } \\
\text { Change }\end{array}$ \\
\hline New England Total & 490 & 639 & 753 & 1,129 & 1,875 & -39.8 \\
\hline Connecticut & 38 & 154 & 317 & 191 & 559 & -65.8 \\
\hline Maine & 11 & & 16 & 11 & 58 & -81.6 \\
\hline Massachusetts & 226 & 217 & 287 & 444 & 833 & -46.7 \\
\hline New Hampshire & 209 & 268 & 133 & 476 & 423 & 126 \\
\hline Rhode Island & & - & • & & . & NM \\
\hline Vermont & 7 & - & . & 7 & 1 & NM \\
\hline Middle Atlantic Total & 6,979 & 6,815 & 7,876 & 13,794 & 16,009 & -13.8 \\
\hline New Jersey & 259 & 277 & 289 & 536 & 649 & -17.5 \\
\hline New York & 2.887 & 2.759 & 3.044 & 5.646 & 6.069 & -7.0 \\
\hline Pennsylvanıa & 3,833 & 3,779 & 4,544 & 7,612 & 9,291 & -18.1 \\
\hline East North Centra! Total & 27,273 & 26,555 & 29,168 & 53,828 & 55,825 & -3.6 \\
\hline Illinois & 4.922 & 5.231 & 4.719 & 10.153 & 9,975 & 1.8 \\
\hline Indiana & 8,247 & 8.531 & 9.157 & 16,778 & 18.221 & -7.9 \\
\hline Michigan & 5.754 & 5.181 & 6.076 & 10,935 & 10,151 & 7.7 \\
\hline$\ldots \ldots \ldots$ & 3.358 & 3,232 & 4,704 & 6.590 & 8,670 & -24.0 \\
\hline Wisconsin & 4.992 & 4,381 & 4.513 & 9,372 & 8,807 & 6.4 \\
\hline West North Central Total & 18,067 & 19,795 & 18,286 & 37,862 & 38,387 & -1.4 \\
\hline lowa & 3.210 & 4.070 & 3,345 & 7.280 & 6,769 & 7.5 \\
\hline$\ldots . . . .$. & 3.373 & 3.477 & 3,993 & 6,849 & 8,321 & -17.7 \\
\hline Minnesota ... ............. & 4.299 & 4,399 & 4.399 & 8,699 & 9,202 & -5.5 \\
\hline Missouri $\ldots \ldots$ & 4.174 & 4,236 & 3,738 & 8,409 & 7.961 & 5.6 \\
\hline Nebraska $\ldots$ & 1,973 & 2.304 & 2,035 & 4.278 & 4,281 & -.1 \\
\hline North Dakota & 408 & 661 & 378 & 1.069 & 902 & 18.4 \\
\hline South Dakota. & 630 & 648 & 398 & 1,278 & 950 & 34.5 \\
\hline South Atlantic Total & 24,321 & 26,880 & 26,051 & 51,201 & 54,135 & -5.4 \\
\hline Delaware . . . . & 530 & 572 & 564 & 1.102 & 1.180 & -6.6 \\
\hline District of Columbia & 12 & 23 & 12 & 35 & 33 & 6.8 \\
\hline Florida & 2.591 & 2.898 & 2.715 & 5.489 & 5,522 & -6 \\
\hline$\ldots \ldots \ldots$ & 4.930 & 5.802 & 6.359 & 10,732 & 12,694 & -15.5 \\
\hline$\ldots \ldots \ldots$ & 2.322 & 2,200 & 2,402 & 4,522 & 4.931 & -8.3 \\
\hline North Carolina $\ldots \ldots \ldots \ldots$ & 4,686 & 5.610 & 5.649 & 10,295 & 11,798 & -12.7 \\
\hline South Carolina & 2.785 & 2.496 & 2,529 & 5,280 & 5.473 & -3.5 \\
\hline Virginia $\ldots . . .$. & 2.504 & 3.452 & 2,321 & 5,956 & 4,935 & 20.7 \\
\hline West Virginia $\ldots$ & 3,961 & 3.828 & 3.500 & 7,789 & 7,570 & 2.9 \\
\hline East South Central Total & 9,558 & 8,433 & 10,167 & 17,990 & 20,503 & -12.3 \\
\hline Alabama & 2.675 & 2.520 & 2,733 & 5.195 & 5,625 & -7.6 \\
\hline Kentucky & 2.824 & 2.900 & 3.778 & 5.724 & 7,896 & -27.5 \\
\hline Mississippi & 581 & 493 & 567 & 1,074 & 1,086 & -1.1 \\
\hline Tennessee & 3,477 & 2.520 & 3,089 & 5.997 & 5,896 & 1.7 \\
\hline West South Central Total & 21,166 & 24,421 & 22,643 & 45,587 & 46,186 & -1.3 \\
\hline Arkansas & 3.133 & 3.076 & 2,582 & 6,210 & 5,111 & 21.5 \\
\hline Louisiana. & 934 & 1,109 & 1.729 & 2.043 & 3,903 & -47.6 \\
\hline$. \ldots \ldots+\ldots+\ldots$ & 3.752 & 4,084 & 3.110 & 7.837 & 7,100 & 10.4 \\
\hline$\ldots$ & 13.346 & 16.152 & 15.222 & 29,498 & 30,072 & -1.9 \\
\hline Mountain Total & 12,883 & 14,175 & 12,263 & 27,059 & 25,908 & 4.4 \\
\hline Arizona & 4,319 & 4,173 & 3,792 & 8,492 & 8,065 & 5.3 \\
\hline Colorado & 2.609 & 3,055 & 2,967 & 5,664 & 5,870 & -3.5 \\
\hline$\ldots \ldots \ldots \ldots$ & 67 & 78 & 91 & 145 & 118 & 22.8 \\
\hline$\ldots+\ldots$ & 110 & 440 & 99 & $55 !$ & 303 & 82.0 \\
\hline$\ldots$ & 809 & 898 & 604 & 1,708 & 1,568 & 89 \\
\hline New Mexico & 158 & 253 & 140 & 410 & 372 & 10.2 \\
\hline$\ldots \ldots \ldots \ldots$ & 1.963 & 1,943 & 1,739 & 3,907 & 3,473 & 12.5 \\
\hline Wyoming & 2.848 & 3.335 & 2,832 & 6,183 & 6.139 & 7 \\
\hline Pacific Total & 2,415 & 2,731 & 965 & 5,146 & 2,117 & 143.0 \\
\hline$\ldots \ldots \ldots+\ldots$ & 142 & 186 & 130 & 328 & 309 & 6.2 \\
\hline$\ldots \ldots \ldots$ & 722 & 700 & 724 & 1.422 & 1,379 & 3.1 \\
\hline Oregon & 455 & 607 & - & 1.063 & . & NM \\
\hline Washington & 1.096 & 1.237 & 111 & 2.333 & 429 & 444.3 \\
\hline U.S. Total & 123,152 & 130,444 & 128,173 & 253,596 & 260,944 & -2.8 \\
\hline
\end{tabular}

- Quanitity is less than 500 short tons or percent is less than 05

1.M Not meaningful as value is greater than or equal to 500

Note. Total may not equal sum of components because of independent rounding

Source Energy Intormation Administration, Form ElA-6. "Coal Distribution Report." 
Table 23. Domestic Distribution of U.S. Coal via River by Origin (Thousand Short Tons)

\begin{tabular}{|c|c|c|c|c|c|c|}
\hline $\begin{array}{l}\text { Coal-Producing State } \\
\text { and Region of Origin }\end{array}$ & $\begin{array}{l}\text { April- } \\
\text { June } \\
1991\end{array}$ & $\begin{array}{c}\text { January- } \\
\text { March } \\
1991\end{array}$ & $\begin{array}{c}\text { April- } \\
\text { June } \\
1990\end{array}$ & 1991 & ar to Dat & $\begin{array}{l}\text { Percent } \\
\text { Change }\end{array}$ \\
\hline Alabama & 626 & 489 & 729 & 1,115 & 1,303 & -144 \\
\hline lilinoss & 4.517 & 4.372 & 4,494 & 8,888 & 8,388 & 60 \\
\hline Indiana & 1,709 & 1.390 & 2,263 & 3,099 & 4,207 & -263 \\
\hline Kentucky Totai & 9.039 & 9.704 & 10.455 & 18,743 & 20,797 & -9.9 \\
\hline Eastern & 3.188 & 3.729 & 4,053 & 6.917 & 8,088 & -145 \\
\hline Western & 5,851 & 5.975 & 6.402 & 11.826 & 12.709 & -69 \\
\hline Montana & 102 & - & 11 & 102 & 11 & NM \\
\hline Ohio $\ldots \ldots \ldots \ldots$ & 2,275 & 1,991 & 2.097 & 4,266 & 4,347 & -1.9 \\
\hline Pennsylvania Total & 1.597 & 1.597 & 1.777 & 3,194 & 3,947 & -19.1 \\
\hline Anthracite & - & - & 8 & - & 11 & - \\
\hline Bituminous & 1,597 & 1.597 & 1.769 & 3.194 & 3.935 & -18.8 \\
\hline Tennessee & 272 & 267 & 172 & 539 & 395 & 365 \\
\hline Virgınia & 547 & 507 & 785 & 1.054 & 1,370 & -23.1 \\
\hline West Virginia Total & 7.686 & 8.357 & 8.191 & 16,043 & 17,092 & -6.1 \\
\hline Northern & 3.624 & 4,240 & 3,788 & 7,865 & 8.304 & -53 \\
\hline Southern & 4,062 & 4,116 & 4,403 & 8.178 & 8.788 & -6.9 \\
\hline 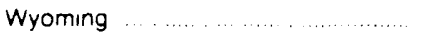 & 3.659 & 3,137 & 3.057 & 6.796 & 5.523 & 23.1 \\
\hline Appalachian Total & 16,192 & 16,938 & 17,803 & 33,129 & 36,542 & -9.3 \\
\hline Interior Total .......... & 12,077 & 11,737 & 13,159 & 23,814 & 25,304 & -5.9 \\
\hline Western Total & 3,761 & 3,137 & 3,068 & 6,898 & 5,534 & 24.6 \\
\hline $\begin{array}{l}\text { East of the Miss. River } \\
\text { West of the Miss. River }\end{array}$ & $\begin{array}{r}28,269 \\
3,761\end{array}$ & $\begin{array}{r}28,674 \\
3,137\end{array}$ & $\begin{array}{r}30,962 \\
3,068\end{array}$ & $\begin{array}{r}56,943 \\
6,898\end{array}$ & $\begin{array}{r}61,846 \\
5,534\end{array}$ & $\begin{array}{r}-7.9 \\
24.6\end{array}$ \\
\hline U.S. Total & 32,030 & 31,812 & 34,030 & 63,841 & 67,381 & -5.3 \\
\hline
\end{tabular}

NM Not meaningful as value is greater than or equal to 500

Note Total may not equal sum of components because of independent rounding

Source Energy Intormation Administration. Form E|A-6. "Coal D stribution Report." 
Table 24. Domestic Distribution of U.S. Coal via River by Destination (Thousand Short Tons)

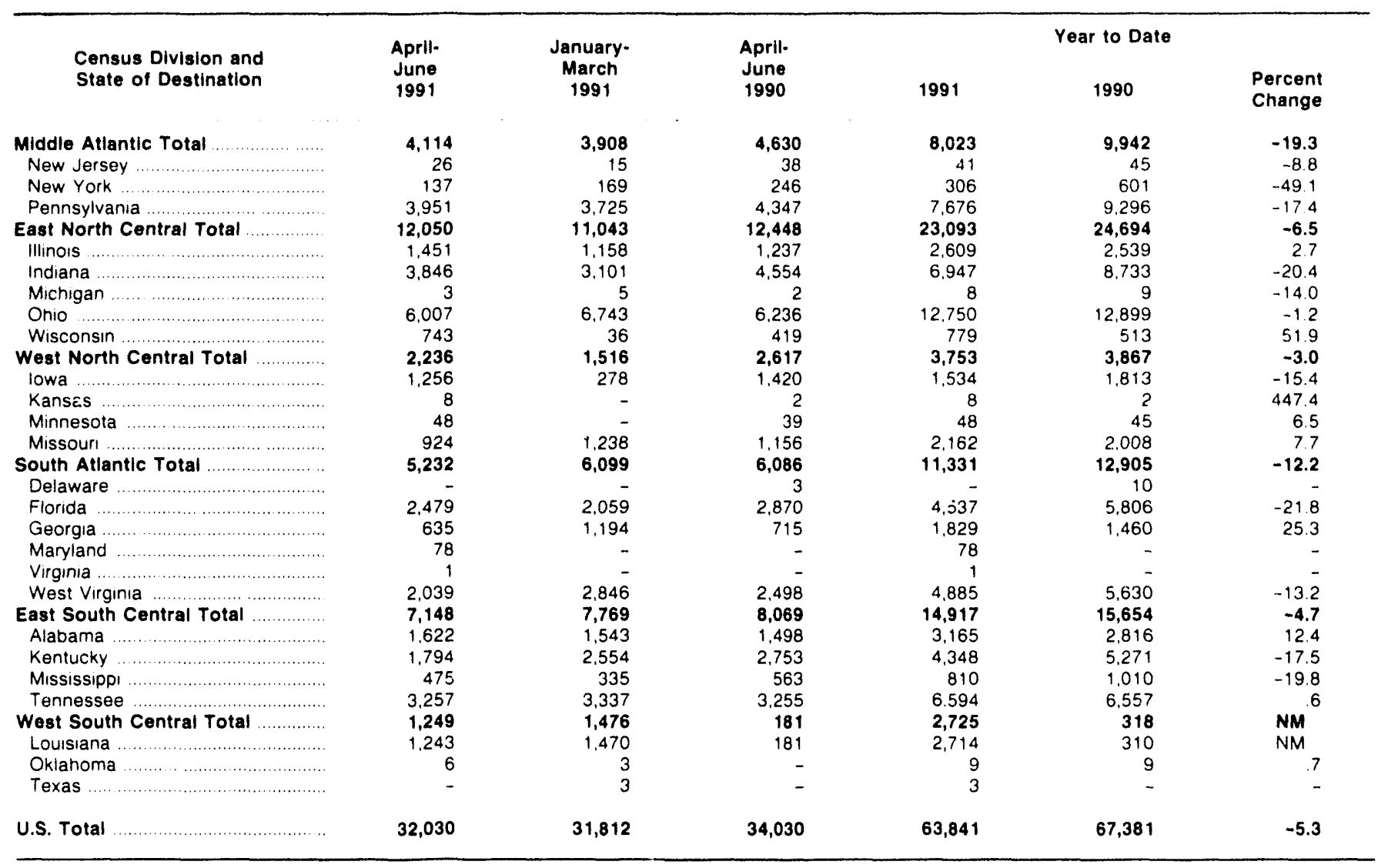

NM Not meaningful as value is greater than or equal to 500 .

Note: Total may not equal sum of components because of independent rounding.

Source: Energy Information Administration. Form E|A-6. "Coal Distribution Report." 
Table 25. Domestic Distribution of U.S. Coal via the Great Lakes by Origin

(Thousand Short Tons)

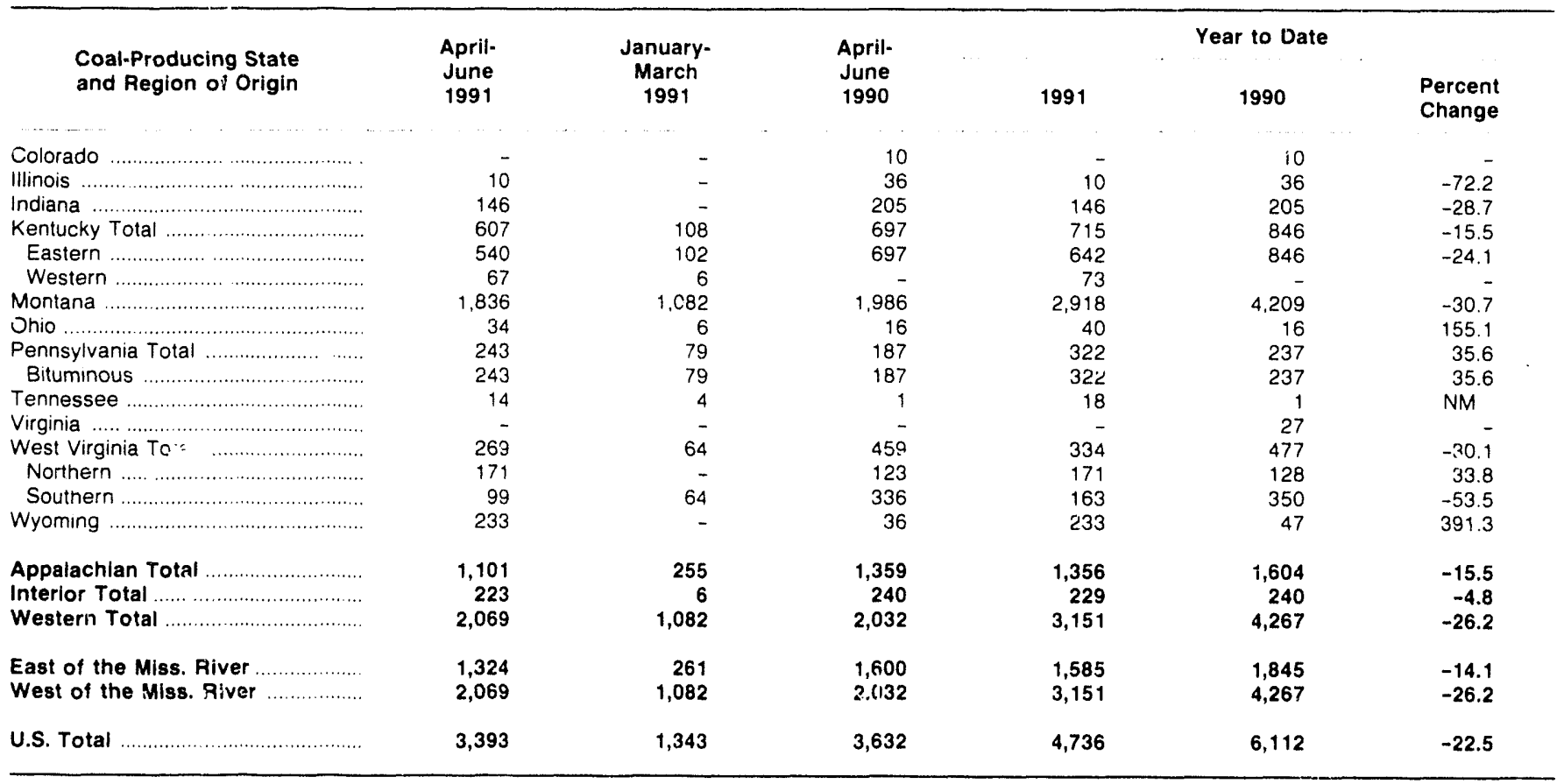

NM Not meaninuful as value is greater than or equal to 500 .

Note: Total may not equal sum of components because o! independent rounding.

Source: Energy Information Administration. Form E|A-6, "Coal Distribution Report."

Table 26. Domestic Distribution of U.S. Coal via the Great Lakes by Destination (Thousand Short Tons)

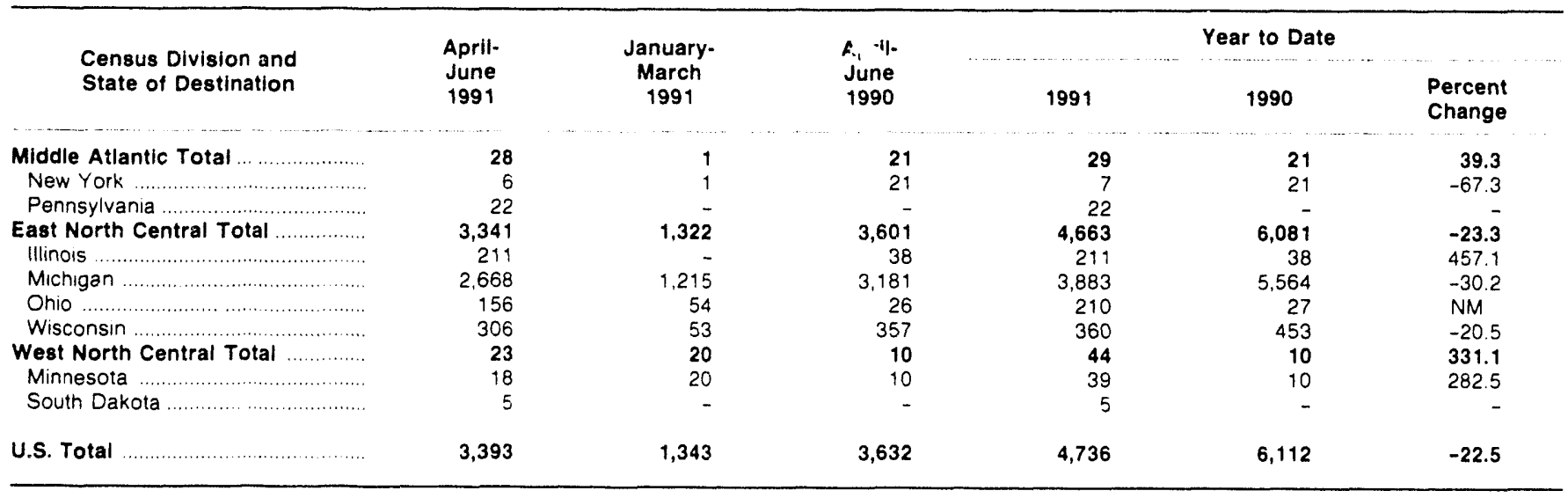

NM Not meaningful as value is greater than or equal to 500 .

Note: Total may not equal sum of components because of independent rounding.

Source: Energy Intormation Administration. Form ElA.6. "Coal Distribution Report. 
Table 27. Domestic Distribution of U.S. Coal via Tidewater by Origin (Thousand Short Tons)

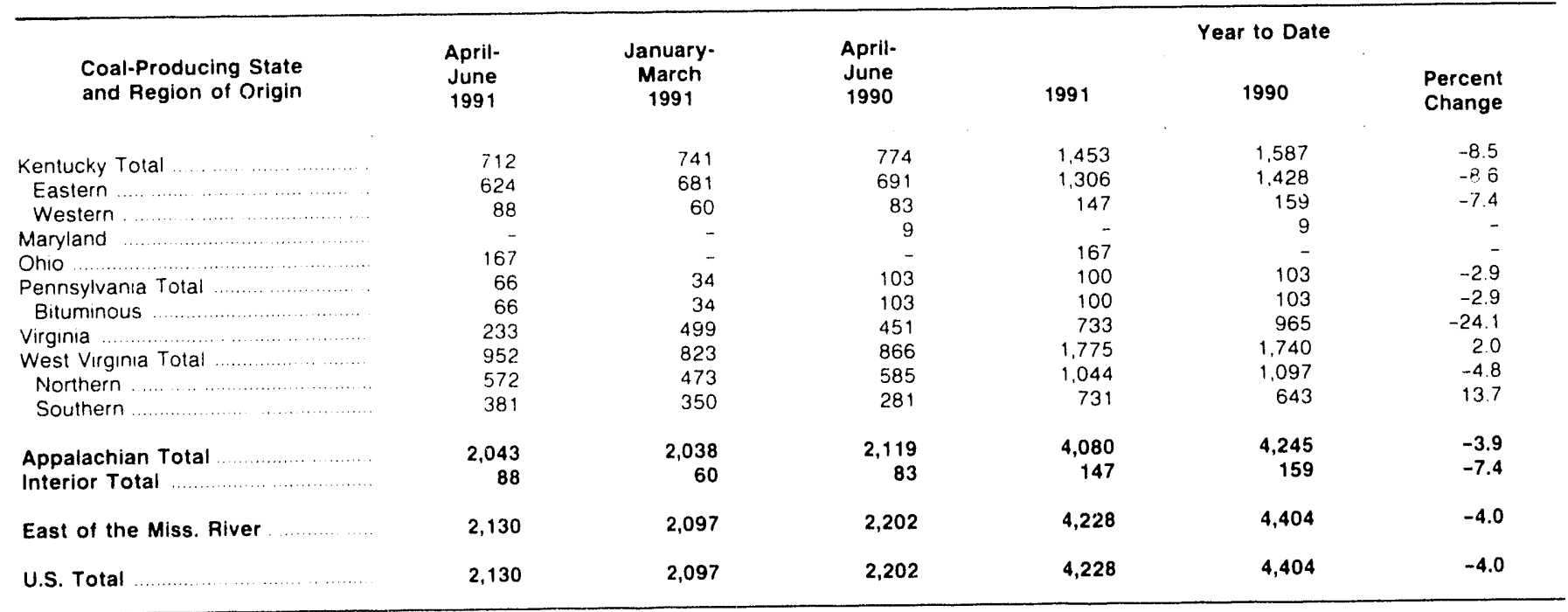

Note: Total may not equal sum of components because of independent rounding.

Source Energy Information Administration. Form ElA.6. "Coal Distribution Report."

Table 28. Domestic Distribution of U.S. Coal via Tidewater by Destination (Thousand Short Tons)

\begin{tabular}{|c|c|c|c|c|c|c|}
\hline \multirow{2}{*}{$\begin{array}{l}\text { Census Division and } \\
\text { State of Destination }\end{array}$} & \multirow{2}{*}{$\begin{array}{l}\text { April. } \\
\text { June } \\
1991\end{array}$} & \multirow{2}{*}{$\begin{array}{c}\text { January- } \\
\text { March } \\
1991\end{array}$} & \multirow{2}{*}{$\begin{array}{l}\text { April- } \\
\text { June } \\
1990\end{array}$} & \multicolumn{3}{|c|}{ Year to Date } \\
\hline & & & & 1991 & 1990 & $\begin{array}{l}\text { Percent } \\
\text { Change }\end{array}$ \\
\hline New England Total .................... & 770 & 886 & 650 & 1,657 & 1,083 & 53.0 \\
\hline 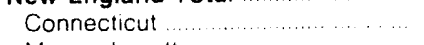 & 202 & 130 & 119 & 332 & 122 & 172.9 \\
\hline 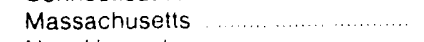 & 439 & 656 & 524 & 1,096 & 944 & 16.1 \\
\hline 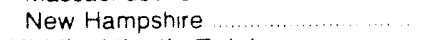 & 129 & 100 & 7 & 229 & 17 & NM \\
\hline Middle Atlantic Total . . ............... & 112 & 313 & 180 & 424 & 466 & -9.0 \\
\hline 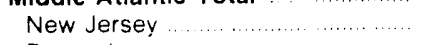 & 112 & 279 & 180 & 391 & 466 & -16.2 \\
\hline 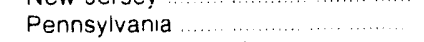 & - & 34 & - & 34 & - & - \\
\hline South Atlantic Total & 1,249 & 894 & 1,368 & 2,143 & 2,851 & -24.8 \\
\hline Florida & 748 & 606 & 601 & 1,354 & 1,261 & 7.4 \\
\hline 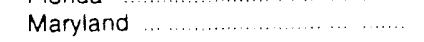 & 394 & 173 & 553 & 567 & 1,047 & -45.9 \\
\hline Virginia & 107 & 115 & 213 & 222 & 543 & -59.0 \\
\hline West South Central Total ........... & - & 4 & 5 & 4 & 5 & -7.3 \\
\hline 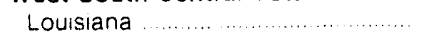 & - & 4 & 5 & 4 & 5 & -7.3 \\
\hline U.S. Total & 2,130 & 2,097 & 2,202 & 4,228 & 4,404 & -4.0 \\
\hline
\end{tabular}

NM Not meaningful as value is greater than or equal to 500 .

Note: Total may not equal sum of components because of independent rounding.

Source: Energy Information Administration, Form EIA.6. "Coal Distribution Report." 
Table 29. Domestic Distribution of U.S. Coal via Truck by Origin (Thousand Short Tons)

\begin{tabular}{|c|c|c|c|c|c|c|}
\hline \multirow{2}{*}{$\begin{array}{l}\text { Coal-Producing State } \\
\text { and Region of Origin }\end{array}$} & \multirow{2}{*}{$\begin{array}{l}\text { April- } \\
\text { June } \\
1991\end{array}$} & \multirow{2}{*}{$\begin{array}{l}\text { January- } \\
\text { March } \\
1991\end{array}$} & \multirow{2}{*}{$\begin{array}{l}\text { April- } \\
\text { June } \\
1990\end{array}$} & \multicolumn{3}{|c|}{ Year to Date } \\
\hline & & & & 1991 & 1990 & $\begin{array}{l}\text { Percent } \\
\text { Change }\end{array}$ \\
\hline Alaheme & $2 \pi$ & & & & & \\
\hline Alabama & 2,175 & 2,313 & 2,321 & 4.489 & 4,584 & -2.1 \\
\hline Alaska & 38 & 46 & 34 & 84 & 81 & 3.5 \\
\hline 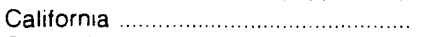 & 8 & - & 11 & 8 & 44 & -81.7 \\
\hline 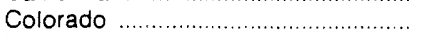 & 1.079 & 1,265 & 1,061 & 345 & 2.218 & 5.7 \\
\hline 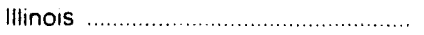 & 1,539 & 1,571 & 1.484 & 111 & 3.109 & .1 \\
\hline 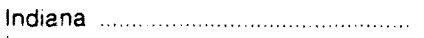 & 1,256 & 1,657 & 1,752 & 2,913 & 3,883 & -25.0 \\
\hline 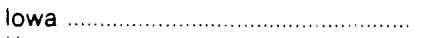 & 80 & 95 & 88 & 175 & 184 & -4.8 \\
\hline 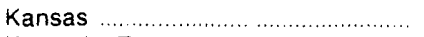 & 109 & 122 & 130 & 230 & 286 & -19.3 \\
\hline 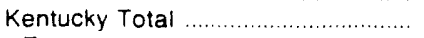 & 2,181 & 2,615 & 2,732 & 4,796 & 5.956 & -19.5 \\
\hline 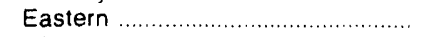 & 1,403 & 1,592 & 1,672 & 2.994 & 3,809 & -214 \\
\hline 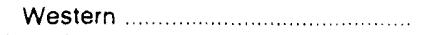 & 778 & 1,023 & 1,060 & 1.802 & 2,147 & -16.1 \\
\hline 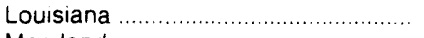 & 82 & 94 & 117 & 177 & 227 & -22.3 \\
\hline 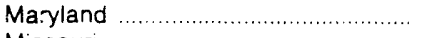 & 177 & 239 & 192 & 416 & 512 & -18.7 \\
\hline 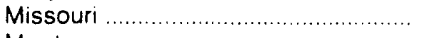 & 99 & 65 & 83 & 164 & 150 & 9.7 \\
\hline 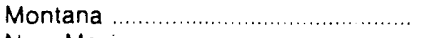 & 81 & 110 & 67 & 191 & 151 & 27.0 \\
\hline New Mexico ...................................... & $\cdot$ & 3 & $\cdot$ & 3 & 2 & 76.0 \\
\hline 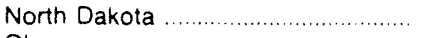 & 779 & 1,116 & 1.029 & 1.895 & 2,190 & -13.5 \\
\hline Ohio & 2.426 & 2,675 & 2.683 & 5.101 & 5.998 & -14.9 \\
\hline 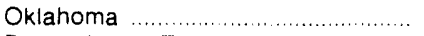 & 390 & 311 & 286 & 700 & 605 & 15.8 \\
\hline 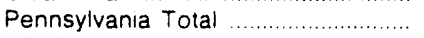 & 3,320 & 3,666 & 4.377 & 6.986 & 9.183 & -23.9 \\
\hline 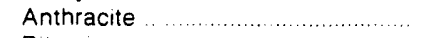 & 424 & 502 & 466 & 926 & 995 & -7.0 \\
\hline 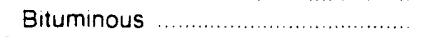 & 2,896 & 3.164 & 3.911 & 6.060 & 8.188 & -26.0 \\
\hline Tennessee ................. & 99 & 149 & 139 & 248 & 321 & -22.6 \\
\hline 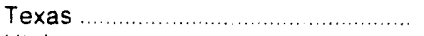 & 3,449 & 3,071 & 2,959 & 6.521 & 5,976 & 9.1 \\
\hline 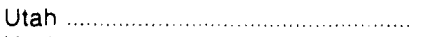 & 1.418 & 1,610 & 1,596 & 3,028 & 3,390 & -10.7 \\
\hline Virginıa $\ldots \ldots \ldots \ldots \ldots \ldots$ & 18 & 23 & 21 & 40 & 58 & -30.3 \\
\hline 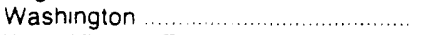 & $\cdot$ & $\cdot$ & 2 & 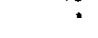 & 12 & -96.9 \\
\hline 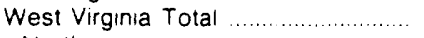 & 1.878 & 1.705 & 2,030 & 3,583 & 4.438 & -19.2 \\
\hline Northern & 1,169 & 1.061 & 1,242 & 2.231 & 2.898 & -23.0 \\
\hline Southern & 709 & 644 & 788 & 1,353 & 1,540 & -12.2 \\
\hline 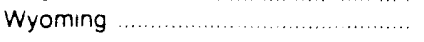 & 631 & 776 & 615 & 1,408 & 1.382 & 1.8 \\
\hline Appalachian Total. & 11,496 & 12,362 & 13,435 & 23,858 & 28,902 & -17.5 \\
\hline Interior Total $\ldots \ldots \ldots \ldots \ldots \ldots$ & 7,783 & 8,010 & 7,960 & 15,793 & 16,566 & -4.7 \\
\hline Western Total & 4,035 & 4,927 & 4,414 & 8,962 & 9,469 & -5.4 \\
\hline $\begin{array}{l}\text { East of the Miss. River } \\
\text { West of the Miss. River } \ldots \ldots \ldots \ldots \ldots \ldots\end{array}$ & $\begin{array}{r}15,070 \\
8,244\end{array}$ & $\begin{array}{r}16,614 \\
8,685\end{array}$ & $\begin{array}{r}17,731 \\
8,078\end{array}$ & $\begin{array}{l}31,684 \\
16,929\end{array}$ & $\begin{array}{l}38,041 \\
16,896\end{array}$ & $\begin{array}{r}-16.7 \\
.2\end{array}$ \\
\hline U.S. Total & 23,314 & 25,299 & 25,810 & 48,613 & 54,937 & -11.5 \\
\hline
\end{tabular}

- Quantity is less than 500 short tons or percent is less than 05 .

Note: Total may not equal sum of components because of independent rounding

Source: Energy Information Administration, Form EIA-6. "Coal Distribution Report." 
Table 30. Domestic Distribution of U.S. Coal via Truck by Destination (Thousand Short Tons)

\begin{tabular}{|c|c|c|c|c|c|c|}
\hline $\begin{array}{l}\text { Census Division and } \\
\text { State of Destination }\end{array}$ & $\begin{array}{c}\text { April- } \\
\text { June } \\
1991\end{array}$ & $\begin{array}{c}\text { January- } \\
\text { March } \\
1991\end{array}$ & $\begin{array}{c}\text { April- } \\
\text { June } \\
1990\end{array}$ & 1991 & ar to Dat & $\begin{array}{l}\text { Percent } \\
\text { Change }\end{array}$ \\
\hline 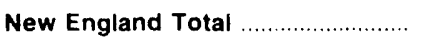 & 19 & 25 & 15 & 44 & 37 & 18.7 \\
\hline 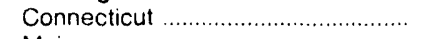 & 1 & 2 & 1 & 3 & 3 & -12.3 \\
\hline Maine & 1 & 1 & $\cdot$ & 2 & 1 & 14.6 \\
\hline Massachusetts & 16 & 16 & 11 & 32 & 26 & 24.3 \\
\hline 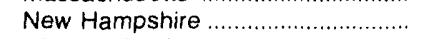 & 1 & 3 & 2 & 5 & 4 & 30.5 \\
\hline 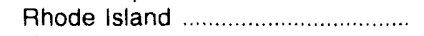 & * & 1 & * & 1 & 1 & -46.1 \\
\hline 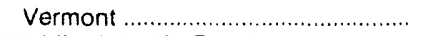 & 1 & 1 & 1 & 2 & 2 & 24.2 \\
\hline Middle Atlantic Total .......................... & 3,254 & 3,482 & 4,241 & 6,736 & 8,858 & -24.0 \\
\hline 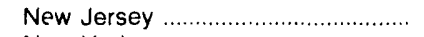 & 3 & 5 & 20 & 8 & 32 & -74.4 \\
\hline 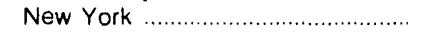 & 243 & 291 & 340 & 534 & 817 & -34.7 \\
\hline 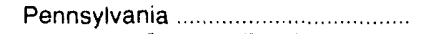 & 3,008 & 3,186 & 3,880 & 6,194 & 8,009 & -22.7 \\
\hline East North Central Total ............... & 5,395 & 6,268 & 6,067 & 11,663 & 13,399 & -13.0 \\
\hline 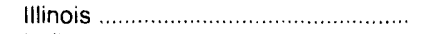 & 1,311 & 1,542 & 1,369 & 2,853 & 2,831 & 8 \\
\hline 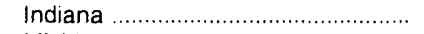 & 1,398 & 1,489 & 1,654 & 2,887 & 3,678 & -21.5 \\
\hline Michigan …....................................... & 99 & 148 & 117 & 247 & 275 & -10.4 \\
\hline 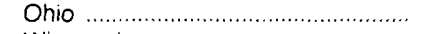 & 2,585 & 3,039 & 2.927 & 5,624 & 6,591 & -14.7 \\
\hline 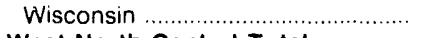 & 2 & 51 & " & 53 & 23 & 130.3 \\
\hline West North Central Total ................. & 1,366 & 1,746 & 1,649 & 3,112 & 3,475 & -10.4 \\
\hline 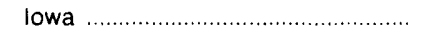 & 87 & 96 & 85 & 183 & 178 & 2.6 \\
\hline 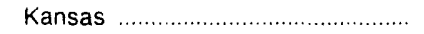 & 47 & 44 & 45 & 91 & 127 & -28.4 \\
\hline 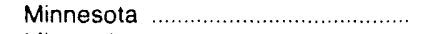 & 16 & 15 & * & 31 & 1 & NM \\
\hline Missouri …........................................ & 321 & 364 & 376 & 685 & 756 & -9.4 \\
\hline 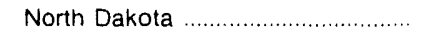 & 779 & 1,116 & 1,029 & 1,895 & 2,190 & -13.5 \\
\hline 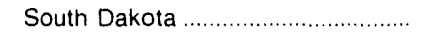 & 116 & 112 & 114 & 227 & 223 & 1.9 \\
\hline 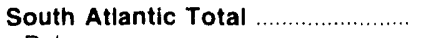 & 1,899 & 1,956 & 2,260 & 3,855 & 5,165 & -25.4 \\
\hline 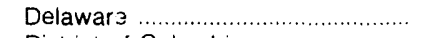 & 1 & 2 & 16 & 3 & 30 & -89.8 \\
\hline District of Columbia .......................... & 1 & 5 & 3 & 6 & 13 & -52.3 \\
\hline Florida & 2 & 2 & 2 & 4 & 4 & 2.2 \\
\hline 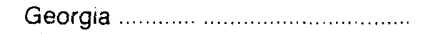 & 33 & 53 & 42 & 86 & 90 & -4.6 \\
\hline 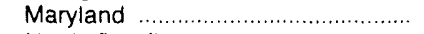 & 109 & 135 & 91 & 244 & 245 & -.4 \\
\hline 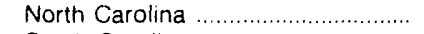 & 87 & 113 & 62 & 200 & 263 & -24.1 \\
\hline 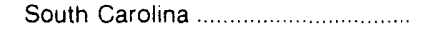 & 34 & 56 & 58 & 90 & 114 & -20.8 \\
\hline 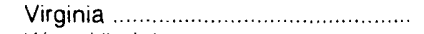 & 143 & 213 & 140 & 356 & 270 & 32.2 \\
\hline West Virginia & 1,488 & 1,378 & 1,847 & 2,866 & 4,136 & -30.7 \\
\hline East South Central Total ................. & 4,330 & 4,676 & 4,938 & 9,006 & 10,128 & -11.1 \\
\hline Alabama …..................................... & 2,362 & 2,301 & 2,308 & 4,663 & 4,568 & 2.1 \\
\hline 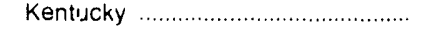 & 1.704 & 2,053 & 2,367 & 3.757 & 4.978 & -24.5 \\
\hline 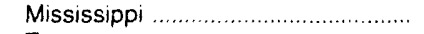 & 2 & 9 & 14 & 11 & 20 & -43.9 \\
\hline 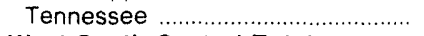 & 262 & 313 & 248 & 575 & 563 & 2.1 \\
\hline West South Central Total ................ & 3,922 & 3,478 & 3,368 & 7,400 & 6,818 & 8.5 \\
\hline 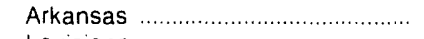 & 29 & 27 & 23 & 56 & 45 & 23.9 \\
\hline 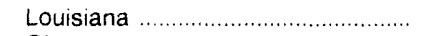 & 82 & 94 & 117 & 177 & 227 & -22.3 \\
\hline Oklahoma & 361 & 285 & 269 & 646 & 569 & 13.7 \\
\hline Texas & 3,450 & 3,071 & 2,959 & 6,521 & 5.977 & 9.1 \\
\hline 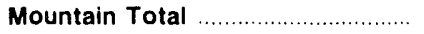 & 3,082 & 3,621 & 3,225 & 6,704 & 6,919 & -3.1 \\
\hline 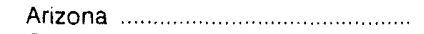 & 3 & 7 & 28 & 10 & 37 & -72.9 \\
\hline 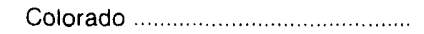 & 1,062 & 1,244 & 1,017 & 2,306 & 2,144 & 7.6 \\
\hline Idaho & 61 & 72 & 50 & 134 & 80 & 66.5 \\
\hline 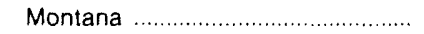 & 79 & 105 & 76 & 184 & 167 & 107 \\
\hline 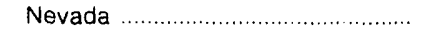 & 11 & 11 & 26 & 22 & 40 & -45.8 \\
\hline 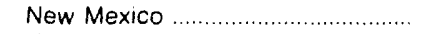 & 15 & 18 & 15 & 33 & 37 & -9.2 \\
\hline Utah & 1,363 & 1,534 & 1,525 & 2,898 & 3,260 & -11.1 \\
\hline 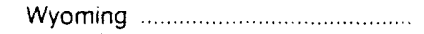 & 488 & 629 & 488 & 1.117 & 1,154 & -3.2 \\
\hline 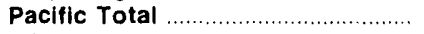 & 47 & 47 & 47 & 94 & 138 & -32.0 \\
\hline 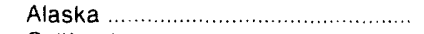 & 38 & 46 & 34 & 84 & 81 & 3.5 \\
\hline 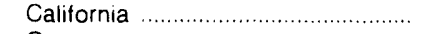 & 8 & $\cdot$ & 11 & 9 & 45 & -80.5 \\
\hline 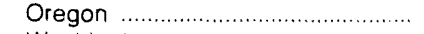 & * & 1 & - & 1 & 1 & 16.0 \\
\hline 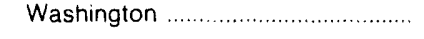 & * & 1 & 2 & 1 & 12 & -93.2 \\
\hline 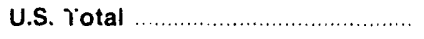 & 23,314 & 25,299 & 25,810 & 48,613 & 54,937 & -11.5 \\
\hline
\end{tabular}

- Quantity is less than 500 short tons or percent is less than .05

NM Not meaningful as value is greater than or equal to 500 .

Note: Total may not equal sum of components because of independent rounding.

Source: Energy Information Administration, Form EIA.6. "Coal Distribution Report." 


\section{Table 31. Domestic Distribution of U.S. Coal via Tramway, Conveyor, and Slurry Pipeline by Origin}

(Thousand Short Tons)

\begin{tabular}{|c|c|c|c|c|c|c|}
\hline $\begin{array}{l}\text { Coal-Producing State } \\
\text { and Region of Origin }\end{array}$ & $\begin{array}{l}\text { April- } \\
\text { June } \\
1991\end{array}$ & $\begin{array}{l}\text { January- } \\
\text { March } \\
1991\end{array}$ & $\begin{array}{l}\text { April- } \\
\text { June } \\
1990\end{array}$ & 1991 & ar to Dat & $\begin{array}{l}\text { Percent } \\
\text { Change }\end{array}$ \\
\hline Alabama & 397 & 458 & 345 & 855 & 789 & 8.4 \\
\hline 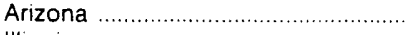 & 1,240 & 1,336 & 627 & 2,575 & 1,774 & 45.1 \\
\hline 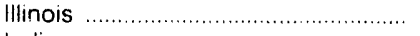 & 586 & 651 & 560 & 1,237 & 1.196 & 3.4 \\
\hline 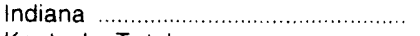 & 155 & 198 & 159 & 352 & 307 & 14.7 \\
\hline 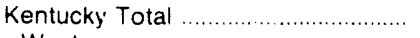 & 562 & 713 & 918 & 1,275 & 2,002 & -36.3 \\
\hline 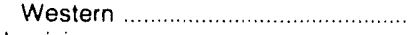 & 562 & 713 & 918 & 1,275 & 2,002 & -36.3 \\
\hline 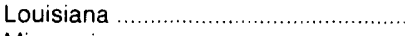 & 498 & 612 & 737 & 1,111 & 1,274 & -12.8 \\
\hline 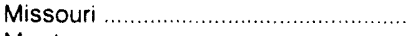 & 361 & 460 & 551 & 821 & 1.187 & -30.8 \\
\hline 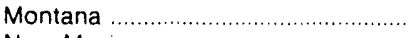 & 1,792 & 2,605 & 1,760 & 4,397 & 4,371 & .6 \\
\hline 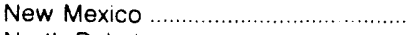 & 2,692 & 2,663 & 3,951 & 5,355 & 7,246 & -26.1 \\
\hline 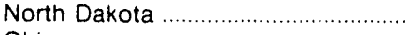 & 4,947 & 5,559 & 5,175 & 10,506 & 10,800 & -2.7 \\
\hline Ohio & 2,220 & 2,332 & 2,389 & 4,551 & 5.192 & -12.3 \\
\hline Pennsylvania Total . ........................ & 2,431 & 2,508 & 2,521 & 4,939 & 5,187 & -4.8 \\
\hline Anthracite ................................... & 88 & 106 & - & 194 & - & - \\
\hline 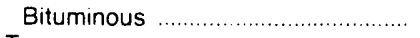 & 2,343 & 2,402 & 2,521 & 4,745 & 5,187 & -8.5 \\
\hline 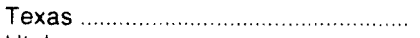 & 4,385 & 4,238 & 4,139 & 8.623 & 8,256 & 4.4 \\
\hline 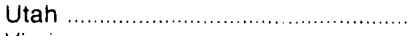 & 462 & 732 & 594 & 1,194 & 1,422 & -16.0 \\
\hline 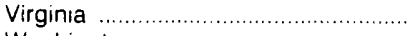 & 240 & 2.40 & 216 & 480 & 425 & 13.0 \\
\hline 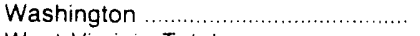 & - & - & 1,219 & - & 2,437 & - \\
\hline 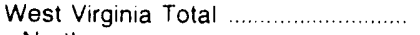 & 952 & 1.330 & 1,494 & 2,282 & 2,751 & -17.0 \\
\hline 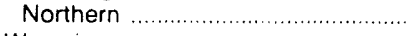 & 952 & 1,330 & 1.494 & 2,282 & 2.751 & -17.0 \\
\hline Wyoming & 2,284 & 2,504 & 2,401 & 4,788 & 4,988 & -4.0 \\
\hline $\begin{array}{l}\text { Appalachian Total } \\
\text { Interior Total } \\
\text { Western Total }\end{array}$ & $\begin{array}{r}6,239 \\
6,547 \\
13,418\end{array}$ & $\begin{array}{r}6,868 \\
6,872 \\
15,398\end{array}$ & $\begin{array}{r}6,965 \\
7,065 \\
15,727\end{array}$ & $\begin{array}{l}13,107 \\
13,419 \\
28,816\end{array}$ & $\begin{array}{l}14,342 \\
14,223 \\
33,040\end{array}$ & $\begin{array}{r}-8.6 \\
-5.7 \\
-12.8\end{array}$ \\
\hline $\begin{array}{l}\text { East of the Miss. River } \ldots \ldots \ldots \ldots \ldots \ldots \\
\text { West of the Miss. River } \ldots \ldots \ldots \ldots \ldots \ldots\end{array}$ & $\begin{array}{r}7,542 \\
18,662\end{array}$ & $\begin{array}{r}8,429 \\
20,709\end{array}$ & $\begin{array}{r}8,602 \\
21,154\end{array}$ & $\begin{array}{l}15,971 \\
39,371\end{array}$ & $\begin{array}{l}17,848 \\
43,757\end{array}$ & $\begin{array}{l}-10.5 \\
-10.0\end{array}$ \\
\hline (n) & 26,204 & 29,137 & 29,757 & 55,342 & 61,605 & -10.2 \\
\hline
\end{tabular}

Note: Total may not equal sum of components because of irıdependent rounding.

Source: Energy Information Administration. Form EIA-6. "Coal Distribution Report." 


\section{Table 32. Domestic Distribution of U.S. Coal via Tramway, Conveyor, and Slurry Pipeline by Destination}

(Thousand Short Tons)

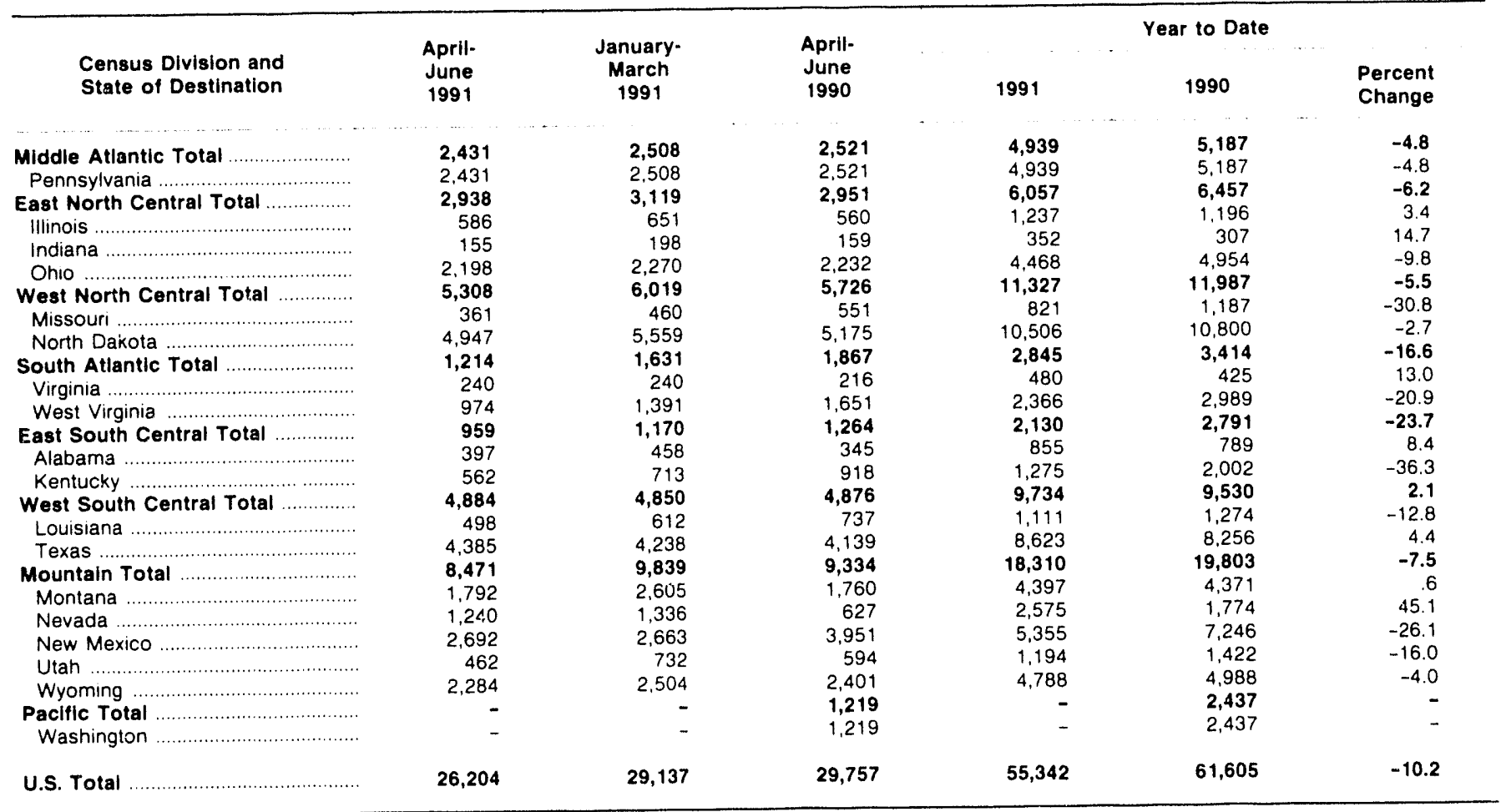

Note: Total may not equal sum of components because of independent rounding. Source: Energy Information Administration, Form EIA-6, "Coal Distribution Report." 


\section{Domestic Destinations}

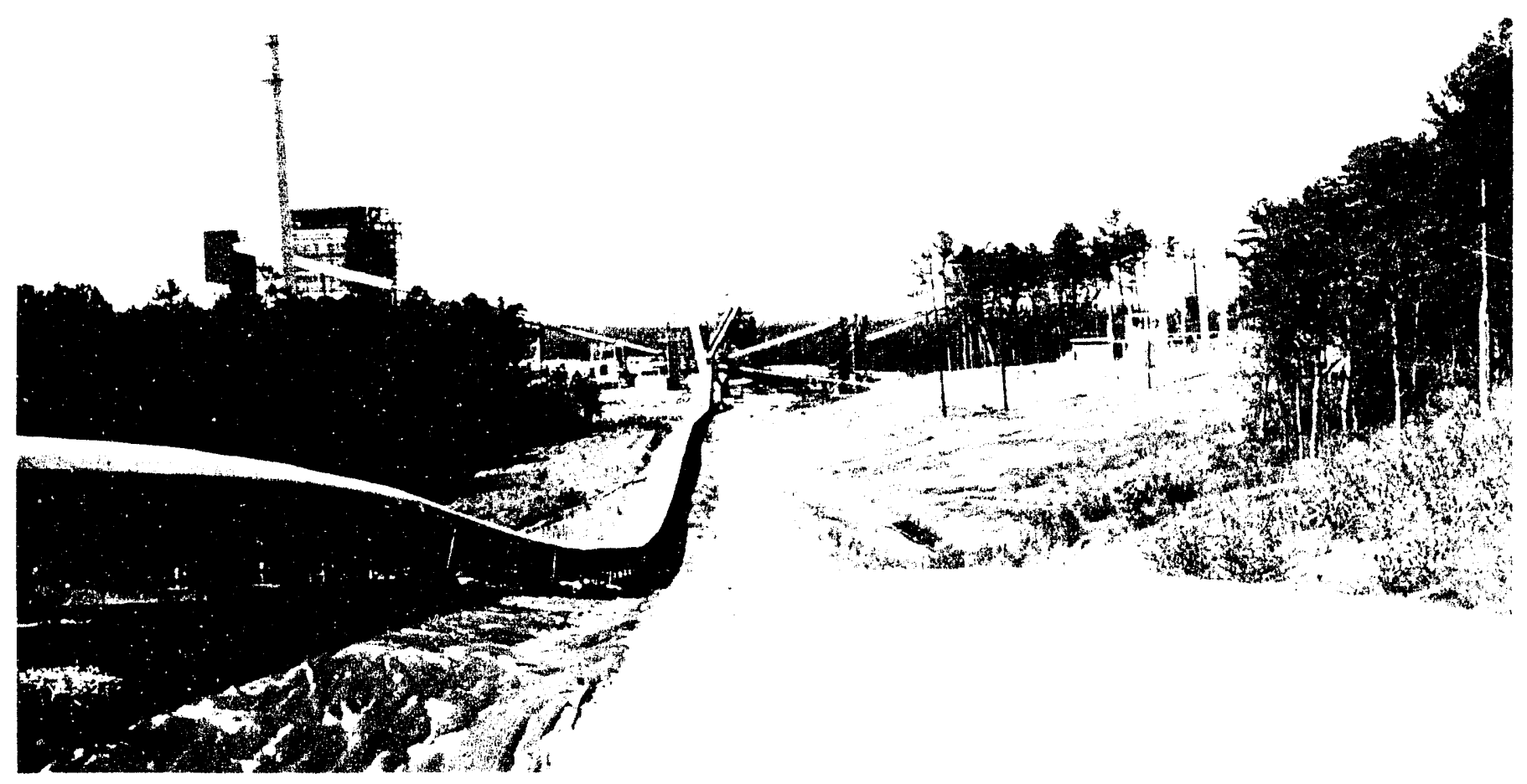

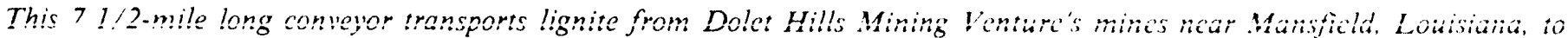
Central Louisiana Electric Company's facilities. 
Figure 6. Domestic Distribution of U.S. Coal by Leading State Destination, JanuaryJune 1991

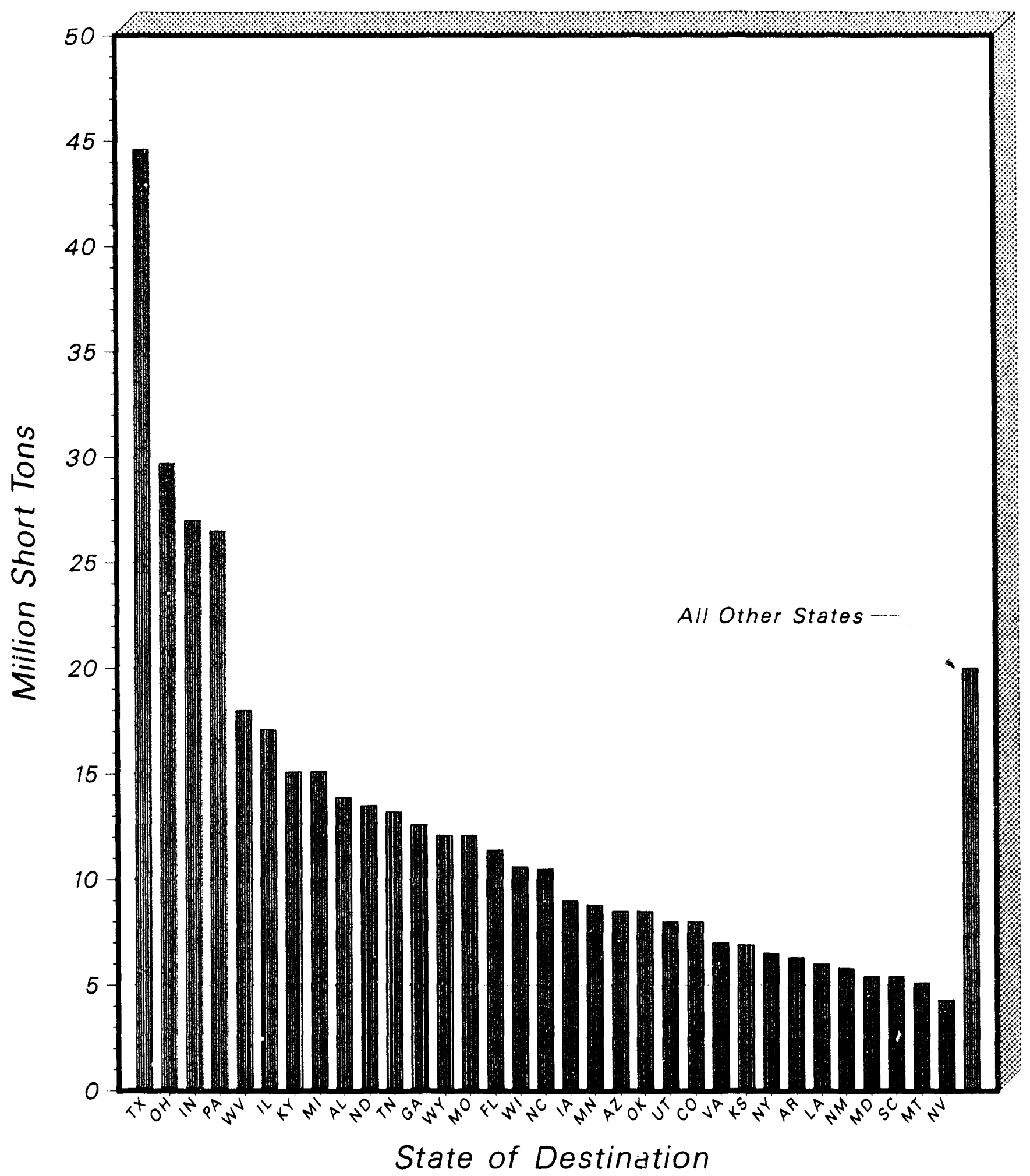

Source: Energy Information Administration, Form ElA-6, "Coal Distribution Report." 
Table 33. Domestic Distribution of U.S. Coal by Origin State, Consumer, Destination and Method of Transportation, January-June 1991

(Thousand Short Tons)

\begin{tabular}{|c|c|c|c|c|c|}
\hline $\begin{array}{l}\text { State of Destination } \\
\text { by Method of Transportation }\end{array}$ & $\begin{array}{l}\text { Electric } \\
\text { Utilities }\end{array}$ & $\begin{array}{l}\text { Coke } \\
\text { Plants }\end{array}$ & $\begin{array}{c}\text { Industrlal } \\
\text { Plants } \\
\text { (Except Coke) }\end{array}$ & $\begin{array}{c}\text { Residential } \\
\text { and Commercial }\end{array}$ & Total \\
\hline & \multicolumn{5}{|c|}{ ORIGIN: ALABAMA } \\
\hline Alabama & 8,447 & 640 & 761 & 4 & 9,853 \\
\hline (2, & 3,069 & 152 & 134 & - & 3,355 \\
\hline River ..... & 1,026 & - & 90 & - & 1,115 \\
\hline Truck & 3,498 & 488 & 479 & 4 & 4,469 \\
\hline Tramway, Conveyor, and Slurry Pipeline ...... & 855 & - & - & - & 855 \\
\hline 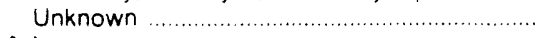 & - & - & 59 & - & 59 \\
\hline Arkansas & - & - & 3 & - & 3 \\
\hline .................. & - & - & 3 & - & 3 \\
\hline 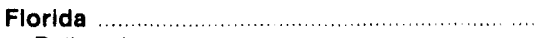 & - & - & 36 & - & 36 \\
\hline … & - & - & 36 & - & 36 \\
\hline 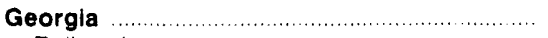 & 39 & - & 2 & - & 41 \\
\hline 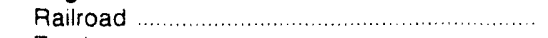 & 39 & - & - & - & 39 \\
\hline 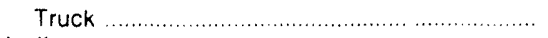 & - & - & 2 & - & 2 \\
\hline Indlana & 6 & - & - & - & 6 \\
\hline 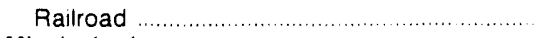 & 6 & - & - & - & 6 \\
\hline 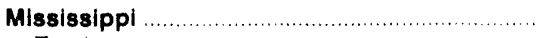 & - & - & 11 & - & 11 \\
\hline 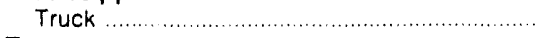 & - & - & 11 & - & 11 \\
\hline 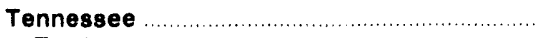 & - & - & 3 & - & 3 \\
\hline Truck … & - & - & 3 & - & 3 \\
\hline 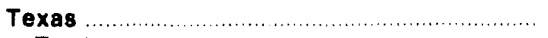 & - & - & * & - & * \\
\hline Truck & - & - & • & - & 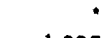 \\
\hline Unknown State & - & - & - & - & 1295 \\
\hline 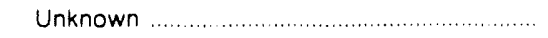 & - & - & - & - & 1295 \\
\hline State Total & 8,493 & 640 & 817 & 4 & 110,249 \\
\hline Railroad & 3,115 & 152 & 170 & - & 3,436 \\
\hline 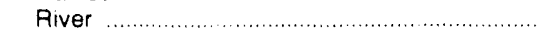 & 1,026 & - & 90 & - & 1,115 \\
\hline 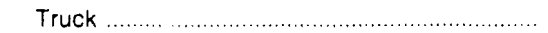 & 3,498 & 488 & 499 & 4 & 4,489 \\
\hline Tramway, Conveyor, and Slurry Pipeline ....... & 855 & - & - & - & 855 \\
\hline 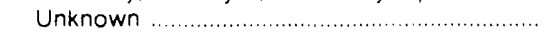 & - & - & 59 & - & 1354 \\
\hline
\end{tabular}
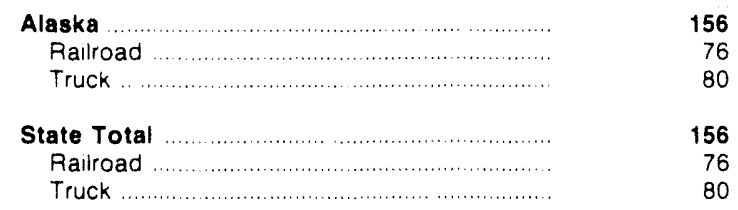

ORIGIN: ALASKA

\begin{tabular}{|c|c|}
\hline Arizona & 3,787 \\
\hline Railroad & 3,787 \\
\hline Nevada & 2,575 \\
\hline Tramway, Conveyor, and Slurry Pipeline & 2.575 \\
\hline State Total & 6,362 \\
\hline 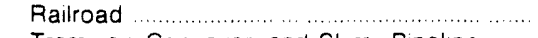 & 3,787 \\
\hline Tramway, Conveyor, and Slurry Pipelıne ....... & 2,575 \\
\hline
\end{tabular}

ORIGIN: ARIZONA

412
328
84
412
328
84

\section{Callfornia
Truck}

Trotal

Truck

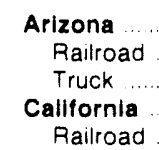

See footnotes at end of table 
Table 33. Domestic Distribution of U.S. Coal by Origin State, Consumer, Destination and Method of Transportation, January-June 1991 (Continued) (Thousand Short Tons)

\begin{tabular}{|c|c|c|c|c|c|}
\hline \multirow[t]{2}{*}{$\begin{array}{l}\text { State of Destination } \\
\text { by Method of Transportation }\end{array}$} & $\begin{array}{l}\text { Electric } \\
\text { Utilities }\end{array}$ & $\begin{array}{l}\text { Coke } \\
\text { Plants }\end{array}$ & $\begin{array}{c}\text { Industrial } \\
\text { Plants } \\
\text { (Except Coke) }\end{array}$ & $\begin{array}{c}\text { Residential } \\
\text { and Commercial }\end{array}$ & Total \\
\hline & \multicolumn{5}{|c|}{ ORIGIN: COLORADO (Continued) } \\
\hline Colorado & 4,898 & - & 295 & 28 & 25,221 \\
\hline 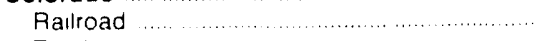 & 2.716 & - & 198 & - & 2,915 \\
\hline 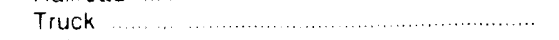 & 2.182 & - & 97 & 27 & 2.305 \\
\hline 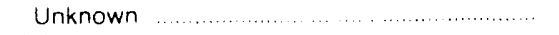 & - & - & - & 1 & 21 \\
\hline 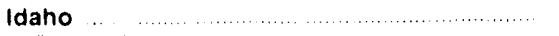 & - & - & 11 & - & 11 \\
\hline 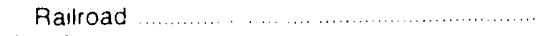 & - & - & 11 & - & 11 \\
\hline 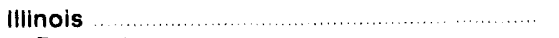 & 315 & - & - & - & 315 \\
\hline 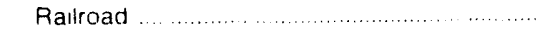 & 315 & - & - &.$\cdot$ & 315 \\
\hline 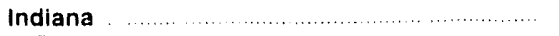 & 429 & - & - & - & 429 \\
\hline 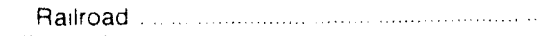 & 429 & - & - & - & 429 \\
\hline 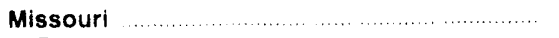 & 208 & - & 5 & - & 213 \\
\hline 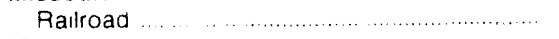 & 208 & - & 5 & - & 213 \\
\hline 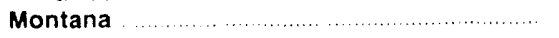 & - & - & 5 & - & 5 \\
\hline 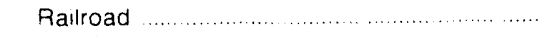 & - & - & 5 & - & 5 \\
\hline 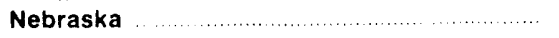 & - & - & 29 & 7 & 36 \\
\hline 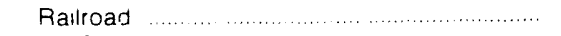 & - & - & 29 & 7 & 36 \\
\hline New Mexico $\ldots \ldots \ldots \ldots$ & 8 & - & 22 & - & 30 \\
\hline 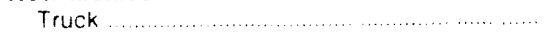 & 8 & - & 22 & - & 30 \\
\hline 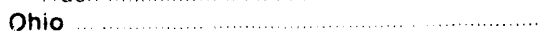 & . & - & - & - & * \\
\hline 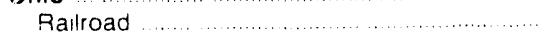 & $\cdot$ & - & - & - & • \\
\hline 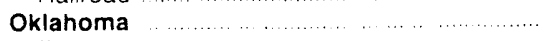 & - & - & 57 & - & 57 \\
\hline 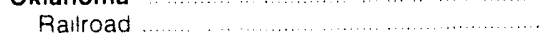 & - & - & 57 & - & 57 \\
\hline Texas $\ldots \ldots \ldots \ldots$ & 808 & - & 243 & - & 1,051 \\
\hline 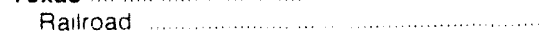 & 808 & - & 243 & - & 1.051 \\
\hline$\ldots \ldots \ldots \ldots \ldots \ldots$ & 742 & 123 & - & - & 865 \\
\hline Raılroad & 742 & 123 & - & - & 865 \\
\hline Washington & - & - & - & 30 & 30 \\
\hline Ralload & - & - & - & 30 & 30 \\
\hline Wyoming & - & - & 32 & - & 32 \\
\hline 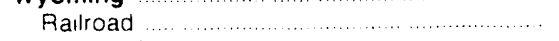 & - & - & 32 & - & 32 \\
\hline Unknown State & - & - & - & - & 11 \\
\hline 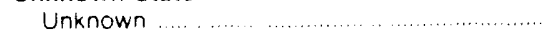 & - & - & - & - & 11 \\
\hline State Total $\ldots \ldots \ldots$ & 7,761 & 123 & 819 & 64 & ${ }^{3} 8,768$ \\
\hline Railroad & 5.571 & 123 & 691 & 36 & 6,422 \\
\hline Truck $\ldots \ldots \ldots \ldots$ & 2.190 & - & 128 & 27 & 2.345 \\
\hline Unknown & - & - & - & 1 & ${ }^{3} 2$ \\
\hline
\end{tabular}

\begin{tabular}{|c|c|}
\hline Alabama & 119 \\
\hline$\ldots \ldots \ldots \ldots \ldots \ldots \ldots \ldots$ & 119 \\
\hline 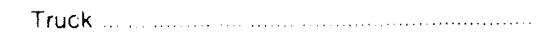 & 1 \\
\hline 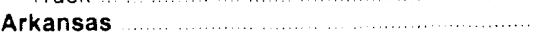 & - \\
\hline … & - \\
\hline$\ldots \ldots \ldots \ldots \ldots \ldots \ldots \ldots \ldots \ldots \ldots \ldots \ldots \ldots \ldots \ldots \ldots \ldots \ldots \ldots \ldots$ & 2,261 \\
\hline$\ldots \ldots \ldots \ldots \ldots \ldots \ldots \ldots \ldots \ldots \ldots \ldots \ldots \ldots \ldots \ldots \ldots$ & 2.261 \\
\hline$\ldots \ldots \ldots \ldots \ldots \ldots \ldots \ldots$ & 1,829 \\
\hline 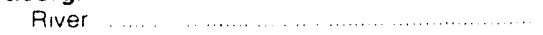 & 1,829 \\
\hline$\ldots \ldots \ldots \ldots \ldots \ldots \ldots$ & 7,879 \\
\hline 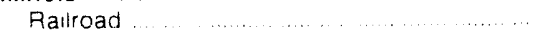 & 4.453 \\
\hline$\ldots \ldots \ldots \ldots \ldots \ldots \ldots \ldots \ldots \ldots \ldots \ldots \ldots \ldots \ldots \ldots$ & 727 \\
\hline 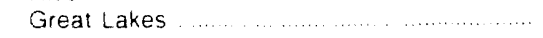 & 10 \\
\hline 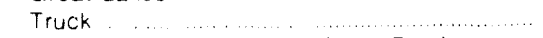 & 1,453 \\
\hline Tramway Conveyor and Slurry Pipeline ...... & 1,237 \\
\hline Unknown & - \\
\hline 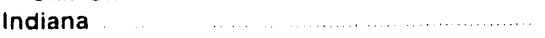 & 4,774 \\
\hline$\ldots \ldots \ldots \ldots \ldots \ldots \ldots \ldots$ & 4,292 \\
\hline$\ldots \ldots \ldots$ & 156 \\
\hline$\ldots \ldots \ldots \ldots \ldots \ldots \ldots \ldots \ldots \ldots \ldots \ldots \ldots \ldots \ldots \ldots \ldots \ldots$ & 326 \\
\hline$\ldots \ldots \ldots \ldots \ldots \ldots$ & 725 \\
\hline Rallroad $\ldots \ldots \ldots \ldots$ & 156 \\
\hline River $\quad \ldots \ldots \ldots \ldots$ & 559 \\
\hline Truck $\quad \ldots \ldots \ldots$ & 10 \\
\hline Kansas $\ldots \ldots \ldots$ & 388 \\
\hline Railioad & 388 \\
\hline
\end{tabular}

ORIGIN: ILLINOIS

$\begin{array}{rrr}- & - & 119 \\ - & - & 119 \\ 54 & - & 1 \\ 54 & - & 54 \\ - & - & 54 \\ - & - & 2,261 \\ - & - & 2,261 \\ - & - & 1,829 \\ 1,278 & - & 1,829 \\ 384 & 85 & 9,242 \\ 25 & - & 4,837 \\ - & - & 753 \\ 857 & - & 10 \\ - & 83 & 2,392 \\ 12 & - & 1,237 \\ 91 & 2 & 14 \\ 41 & - & 5,228 \\ - & - & 4,696 \\ 50 & - & 156 \\ 23 & - & 376 \\ - & 13 & 761 \\ 23 & 13 & 169 \\ - & - & 583 \\ - & - & 10 \\ - & - & 388 \\ & - & 388\end{array}$

See footnotes at end of table 
Table 33. Domestic Distribution of U.S. Coal by Origin State, Consumer, Destination and Method of Transportation, January-June 1991 (Continued) (Thousand Short Tons)

\begin{tabular}{|c|c|c|c|c|c|}
\hline $\begin{array}{l}\text { State of nestination } \\
\text { by Method of Transportation }\end{array}$ & $\begin{array}{l}\text { Electric } \\
\text { Utilities }\end{array}$ & $\begin{array}{l}\text { Coke } \\
\text { Plants }\end{array}$ & $\begin{array}{c}\text { Industrial } \\
\text { Plants } \\
\text { (Except Coke) }\end{array}$ & $\begin{array}{l}\text { Residential } \\
\text { and Commercial }\end{array}$ & Total \\
\hline & \multicolumn{5}{|c|}{ ORIGIN: ILLINOIS (Continued) } \\
\hline Minnesota & 9 & - & - & - & 9 \\
\hline Raitroad $\ldots \ldots \ldots \ldots \ldots$ & 1 & - & - & - & 1 \\
\hline 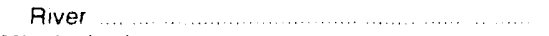 & 8 & - & - & - & 8 \\
\hline$\ldots \ldots \ldots \ldots \ldots \ldots \ldots \ldots \ldots$ & 707 & - & 38 & - & 745 \\
\hline$\ldots \ldots \ldots \ldots \ldots \ldots$ & 707 & - & 38 & - & 745 \\
\hline$\ldots \ldots \ldots \ldots \ldots \ldots \ldots \ldots \ldots \ldots \ldots \ldots \ldots \ldots \ldots \ldots \ldots \ldots \ldots \ldots$ & 5,897 & - & 345 & 61 & 6,303 \\
\hline 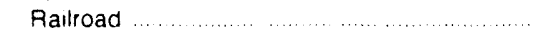 & 4.273 & - & - & - & 4.273 \\
\hline 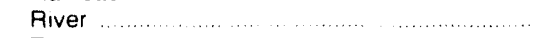 & 1,624 & - & 75 & - & 1.699 \\
\hline Truck $\ldots \ldots \ldots \ldots \ldots$ & - & - & 270 & 61 & 331 \\
\hline 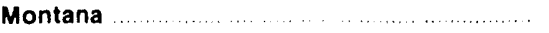 & 201 & - & - & - & 201 \\
\hline 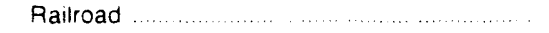 & 201 & - & - & - & 201 \\
\hline Ohio & - & - & $\because$ & - & * \\
\hline$\ldots \ldots \ldots \ldots \ldots \ldots \ldots \ldots \ldots \ldots \ldots \ldots \ldots \ldots \ldots \ldots \ldots \ldots \ldots$ & - & - & $\cdot$ & - & • \\
\hline$\ldots \ldots \ldots \ldots \ldots \ldots \ldots \ldots \ldots \ldots \ldots \ldots \ldots \ldots \ldots \ldots$ & 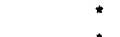 & - & - & * & : \\
\hline 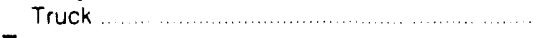 & $\cdot$ & - & - & $\cdot$ & $\cdot$ \\
\hline Tennessee & 664 & - & - & - & 664 \\
\hline 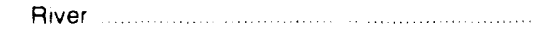 & 664 & - & - & - & 664 \\
\hline Texas $\ldots \ldots \ldots$ & * & - & $\cdot$ & - & * \\
\hline River $\ldots \ldots \ldots \ldots \ldots \ldots \ldots$ & - & - & $\cdot$ & - & • \\
\hline Truck …............................................ & $\cdot$ & - & - & - & * \\
\hline Wisconsin $\ldots \ldots \ldots \ldots$ & 227 & - & 153 & - & 380 \\
\hline Railroad $\ldots \ldots \ldots$ & 155 & - & 153 & - & 308 \\
\hline - & 72 & - & - & - & 72 \\
\hline 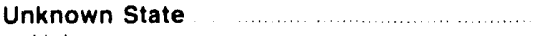 & - & - & - & - & 17 \\
\hline 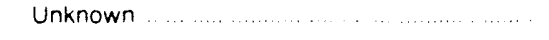 & - & - & - & - & 17 \\
\hline State Total & 25.682 & 363 & 1,982 & 159 & 28,193 \\
\hline …....... $\quad \ldots \ldots$ & 13.919 & 363 & 632 & 13 & 14,926 \\
\hline …........ & 8.727 & - & 161 & - & 8,888 \\
\hline 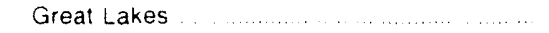 & 10 & - & - & - & 10 \\
\hline$\ldots \quad \ldots \ldots \ldots, \ldots, \quad \ldots \quad \ldots \ldots \ldots \ldots \ldots \ldots$ & 1.790 & - & 1,177 & 144 & 3.111 \\
\hline Tramway. Conveyor and Slurry Pipelıne ....... & 1,237 & - & - & - & 1.237 \\
\hline \multirow[t]{2}{*}{ 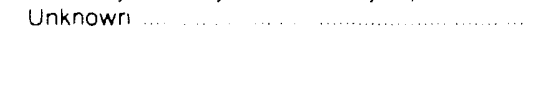 } & - & - & 12 & 2 & +21 \\
\hline & \multicolumn{5}{|c|}{ ORIGIN: INDIANA } \\
\hline Alabama & 85 & - & - & - & 85 \\
\hline 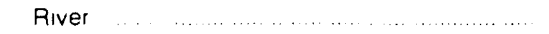 & 85 & - & - & - & 85 \\
\hline$\ldots \ldots \ldots \ldots \ldots \ldots \ldots$ & 111 & - & - & - & 111 \\
\hline 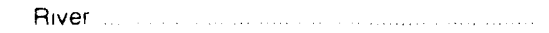 & 111 & - & - & - & 111 \\
\hline 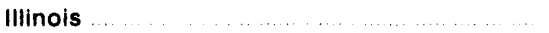 & 738 & - & 322 & 16 & 1,076 \\
\hline Rallroad $\ldots \ldots \ldots$ & 137 & - & + & - & 137 \\
\hline River $\ldots \ldots \ldots$ & 321 & - & 194 & - & 515 \\
\hline Truck & 281 & - & 128 & 16 & 424 \\
\hline Indiana & 8,547 & - & 1,325 & 221 & 10,092 \\
\hline Railroad $\ldots \ldots \ldots$ & 5.914 & - & 788 & - & 6.702 \\
\hline River $\ldots$ & 639 & - & 8 & - & 647 \\
\hline 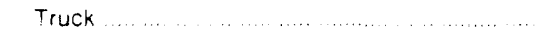 & 1.641 & - & 519 & 220 & 2.380 \\
\hline Tramway. Conveyor, and Slurry Pipelıne & 352 & - & - & - & 352 \\
\hline 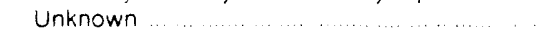 & - & - & 11 & 1 & 11 \\
\hline lowa $\ldots \ldots \ldots \ldots$ & 517 & - & 116 & - & 634 \\
\hline Ralload $\ldots$ & 264 & - & 24 & - & 287 \\
\hline River & 254 & - & 93 & - & 346 \\
\hline Kansas & - & - & 6 & - & 6 \\
\hline Truck & - & - & 6 & - & 6 \\
\hline Kentucky & 1,094 & - & 18 & - & 1,112 \\
\hline Railroad $\ldots$ & 113 & - & - & - & 113 \\
\hline River $\ldots \ldots \ldots$ & 920 & - & - & - & 920 \\
\hline Truck $\ldots \ldots \ldots$ & 61 & - & 18 & - & 79 \\
\hline Michigan & 48 & - & 93 & 3 & 144 \\
\hline Greal Lakes $\ldots \ldots \ldots$ & 48 & - & 92 & - & 139 \\
\hline Truck & - & - & 1 & 3 & 4 \\
\hline Minnesota & 42 & - & 12 & 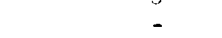 & 54 \\
\hline Rastroad & 42 & - & - & - & 42 \\
\hline River $\ldots$ & - & - & 12 & - & 12 \\
\hline Truck & - & - & $\cdot$ & - & $\cdot$ \\
\hline
\end{tabular}

See footnotes at end of table 

Table 33. Domestic Distribution of U.S. Coal by Origin State, Consumer, Destination
and Method of Transportation, January-June 1991 (Continued)

(Thousand Short Tons)

\begin{tabular}{|c|c|c|c|c|c|}
\hline $\begin{array}{l}\text { State of Destination } \\
\text { by Method of Transportation }\end{array}$ & $\begin{array}{l}\text { Electric } \\
\text { Utilities }\end{array}$ & $\begin{array}{l}\text { Coke } \\
\text { Plants }\end{array}$ & $\begin{array}{c}\text { Industrial } \\
\text { Plants } \\
\text { (Except Coke) }\end{array}$ & $\begin{array}{c}\text { Residential } \\
\text { and Commercial }\end{array}$ & Total \\
\hline & \multicolumn{5}{|c|}{ ORIGIN: INDIANA (Continued) } \\
\hline Missouri & 69 & - & 17 & 2 & 88 \\
\hline River & 69 & - & 2 & 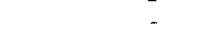 & 71 \\
\hline Truck & $\sim$ & - & 15 & 2 & 17 \\
\hline Ohio & - & - & 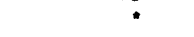 & - & - \\
\hline Truck & - & - & • & - & - \\
\hline Oklahoma & - & - & 2 & - & 2 \\
\hline . & - & - & 2 & - & 2 \\
\hline . & 113 & - & - & - & 113 \\
\hline (............................... & 113 & - & - & - & 113 \\
\hline (................................ & - & - & - & * & * \\
\hline ................. & - & - & - & * & - \\
\hline 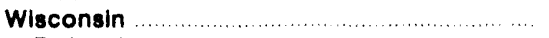 & 1,141 & - & 36 & - & 1,177 \\
\hline 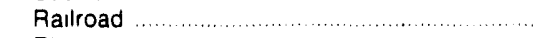 & 861 & - & 30 & - & 890 \\
\hline River & 280 & - & - & - & 280 \\
\hline 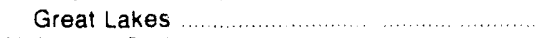 & - & - & 7 & - & 7 \\
\hline Unknown State & - & - & - & - & 1124 \\
\hline Unknown & - & - & - & - & 1124 \\
\hline State Total & 12,505 & - & 1,947 & 242 & 14,818 \\
\hline Railroad & 7,330 & - & 841 & - & 8,172 \\
\hline 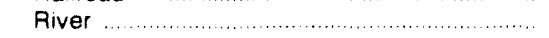 & 2.792 & - & 308 & - & 3,099 \\
\hline 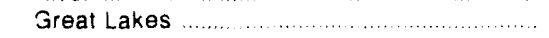 & 48 & - & 98 & - & 146 \\
\hline Truck & 1.983 & - & 689 & 242 & 2.913 \\
\hline Tramway, Conveyor, and Slurry Fipeline ....... & 352 & - & - & - & 352 \\
\hline Unknown ...... & - & - & 11 & 1 & 1135 \\
\hline
\end{tabular}

ORIGIN: IOWA

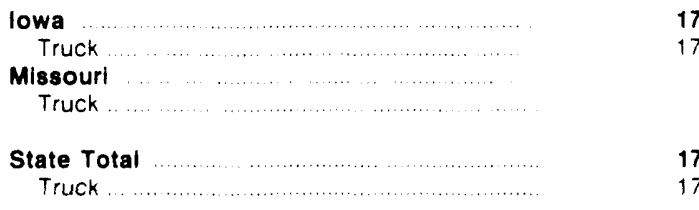

$\begin{array}{rrrrr}172 & - & - & 1 & 173 \\ 172 & - & - & 1 & 173 \\ 2 & - & - & - & 2 \\ 2 & - & - & - & 2 \\ 174 & - & - & 1 & 175 \\ 174 & - & - & 1 & 175\end{array}$

\begin{tabular}{|c|c|}
\hline (n) & $\begin{array}{l}56 \\
56\end{array}$ \\
\hline (2) & 134 \\
\hline 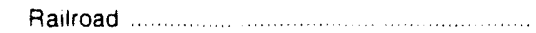 & 27 \\
\hline Truck & 106 \\
\hline State Total & 190 \\
\hline Railroad & 27 \\
\hline Truck. & 16 \\
\hline Alabama & 1,917 \\
\hline$\ldots \ldots \ldots \ldots \ldots \ldots$ & 67 \\
\hline 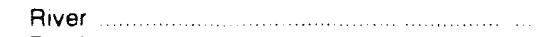 & 1.24 \\
\hline . & \\
\hline Arkansas & \\
\hline Truck ... & \\
\hline Connectlcut $\ldots \ldots \ldots \ldots$ & 47 \\
\hline Railroad $\ldots \ldots \ldots \ldots \ldots$ & 14 \\
\hline 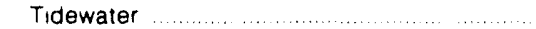 & 33 \\
\hline Delaware $\ldots . . . . .$. & \\
\hline 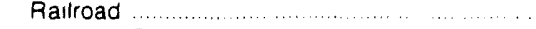 & \\
\hline District of Columbia & \\
\hline Railroad …....................... & \\
\hline Florida & 7,19 \\
\hline . & 4,56 \\
\hline$\ldots \ldots \ldots \ldots \ldots \ldots \ldots \ldots \ldots \ldots \ldots \ldots$ & 2.08 \\
\hline Tidewater & \\
\hline
\end{tabular}

ORIGIN: KANSAS

$\begin{array}{rrr}16 & * & 72 \\ 16 & - & 72 \\ 51 & 1 & 185 \\ - & - & 27 \\ 51 & 1 & 158 \\ 67 & & 258 \\ - & 1 & 27 \\ 67 & 1 & 230\end{array}$

ORIGIN: KENTUCKY, TOTAL

$\begin{array}{rrr}43 & \cdot & 1,960 \\ 43 & - & 717 \\ - & - & 1,242 \\ 1 & - & 1 \\ 5 & - & 5 \\ 5 & - & 5 \\ - & - & 478 \\ - & - & 146 \\ - & - & 772 \\ - & - & 52 \\ -. & - & 52 \\ - & 33 & 33 \\ - & 33 & 33 \\ 204 & - & 7,397 \\ 204 & - & 4.770 \\ - & - & 2.081 \\ - & - & 546\end{array}$

See tootnotes at end of table 
Table 33. Domestic Distribution of U.S. Coal by Origin State, Consumer, Destination and Method of Transportation, January-June 1991 (Continued) (Thousand Short Tons)

\begin{tabular}{|c|c|c|c|c|c|}
\hline $\begin{array}{l}\text { State of Destination } \\
\text { by Method of Transportation }\end{array}$ & $\begin{array}{l}\text { Electric } \\
\text { Utilities }\end{array}$ & $\begin{array}{l}\text { Coke } \\
\text { Plants }\end{array}$ & $\begin{array}{c}\text { Industrial } \\
\text { Plants } \\
\text { (Except Coke) }\end{array}$ & $\begin{array}{c}\text { Residential } \\
\text { and Commercial }\end{array}$ & Total \\
\hline & \multicolumn{5}{|c|}{ ORIGIN: KENTUCKY, TOTAL (Continued) } \\
\hline Georgla & 6,096 & - & 641 & 4 & 6,741 \\
\hline 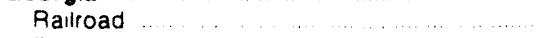 & 6.096 & - & 608 & 4 & 6.708 \\
\hline Truck & 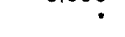 & - & 33 & $\cdot$ & 33 \\
\hline IIIInols $\ldots \ldots$ & 814 & 134 & 202 & - & 1,151 \\
\hline Riulroad & 342 & 7 & 44 & - & 394 \\
\hline rtiver & 456 & 127 & 144 & - & 726 \\
\hline Truck & 16 & - & 15 & - & 31 \\
\hline$\ldots$ & 1,989 & 808 & 10 & - & 2,808 \\
\hline$\ldots$ & 280 & 747 & - & - & 1,027 \\
\hline$\ldots$ & 1,707 & 61 & - & - & 1,768 \\
\hline Truck & 2 & 1 & 10 & . & 13 \\
\hline lowa & 36 & - & 139 & 138 & 313 \\
\hline Railroad & - & - & 1 & - & 1 \\
\hline River & 36 & - & 138 & 138 & 312 \\
\hline Truck & - & - & 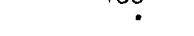 & - & 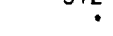 \\
\hline${ }_{1}$ & - & - & 3 & - & 3 \\
\hline (1, & - & - & 3 & - & 3 \\
\hline Kentucky & 10,596 & 50 & 595 & 101 & 211,343 \\
\hline Ralload & 3.791 & 46 & 247 & 18 & 4.102 \\
\hline River & 2,553 & - & 2 & . & 2.555 \\
\hline 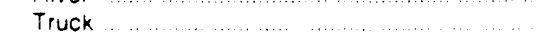 & 2,977 & 4 & 339 & 46 & 3,365 \\
\hline Tramway, Conveyor, and Slurry Pipeline & 1.275 & - & - & - & 1,275 \\
\hline Unknown & - & - & 8 & 37 & 246 \\
\hline Loulsiana & 319 & - & 50 & - & 369 \\
\hline River & 315 & - & 50 & - & 365 \\
\hline Tidewater & 4 & - & - & - & 4 \\
\hline Maryland $\ldots \ldots \ldots$ & 194 & 78 & 87 & - & 359 \\
\hline 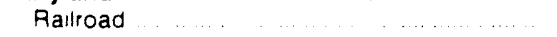 & 51 & - & 87 & - & 138 \\
\hline River $\ldots$ & - & 78 & - & - & 78 \\
\hline Tidewater $\ldots$ & 143 & - & - & - & 143 \\
\hline Massachusetts & 232 & - & 7 & • & 239 \\
\hline Raliroad & 15 & - & 4 & • & 20 \\
\hline Tidewater & 217 & - & - & - & 217 \\
\hline Truck & - & - & 2 & - & 2 \\
\hline Michigan & 3,354 & 4 & 551 & 73 & 3,982 \\
\hline 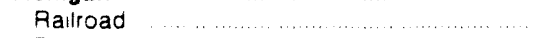 & 3.144 & - & 293 & 72 & 3.509 \\
\hline 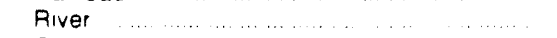 & - & - & 8 & - & 8 \\
\hline Great Lakes & 210 & - & 223 & 1 & 434 \\
\hline Truck & - & 4 & 27 & - & 31 \\
\hline Minnesota & 29 & - & 2 & - & 31 \\
\hline River & 9 & - & 2 & - & 11 \\
\hline 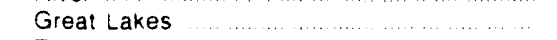 & 20 & - & - & - & 20 \\
\hline Truck & - & - & $\cdot$ & - & $\cdot$ \\
\hline Mississippl $\ldots \ldots \ldots$ & 1,093 & - & 23 & - & 1,116 \\
\hline Ralload & 1.051 & - & - & - & 1.051 \\
\hline River $\ldots$ & 42 & - & 23 & - & 65 \\
\hline$\ldots \ldots \ldots \ldots$ & 324 & - & 60 & • & 384 \\
\hline Railroad $\ldots \ldots \ldots$ & 31 & - & 2 & - & 33 \\
\hline River $\ldots \ldots \ldots \ldots$ & 293 & - & 51 & - & 344 \\
\hline 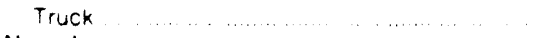 & - & - & 8 & $\cdot$ & 8 \\
\hline New Jersey & 17 & - & 12 & - & 29 \\
\hline Railroad & 17 & - & 12 & - & 29 \\
\hline 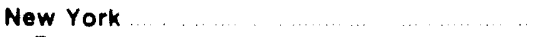 & 768 & 4 & 12 & 17 & 801 \\
\hline Railroad $\ldots \ldots$ & 768 & 4 & 11 & 7 & 790 \\
\hline River $\ldots$ & - & - & - & 9 & 9 \\
\hline Great Lakes . . . . . . . . . & - & - & - & 1 & 1 \\
\hline Truck & - & - & $\cdot$ & - & $\cdot$ \\
\hline North Carolina & 4,754 & - & 778 & 14 & 5,546 \\
\hline Ratroad $\ldots \ldots \ldots \ldots$ & 4.754 & - & 624 & 12 & 5.390 \\
\hline Truck $\ldots \ldots \ldots$ & - & - & 155 & 2 & 157 \\
\hline North Dakota $\ldots \ldots \ldots$ & - & - & 10 & - & 10 \\
\hline Railioad & - & - & in & - & 10 \\
\hline$\ldots-\ldots \ldots-\ldots=\ldots \ldots$ & 4,378 & 252 & 929 & 60 & 5,619 \\
\hline Railroad $\ldots$ & 1.644 & 187 & 570 & 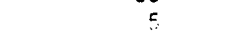 & 2,405 \\
\hline River & 2.660 & 56 & 14 & $\because$ & 2.730 \\
\hline Truck & 75 & 8 & 345 & 55 & 484 \\
\hline
\end{tabular}

See tootnotes at end of table. 

Table 33. Domestic Distribution of U.S. Coal by Origin State, Consumer, Destination
and Method of Transportation, January-June 1991 (Continued) (Thousand Short Tons)

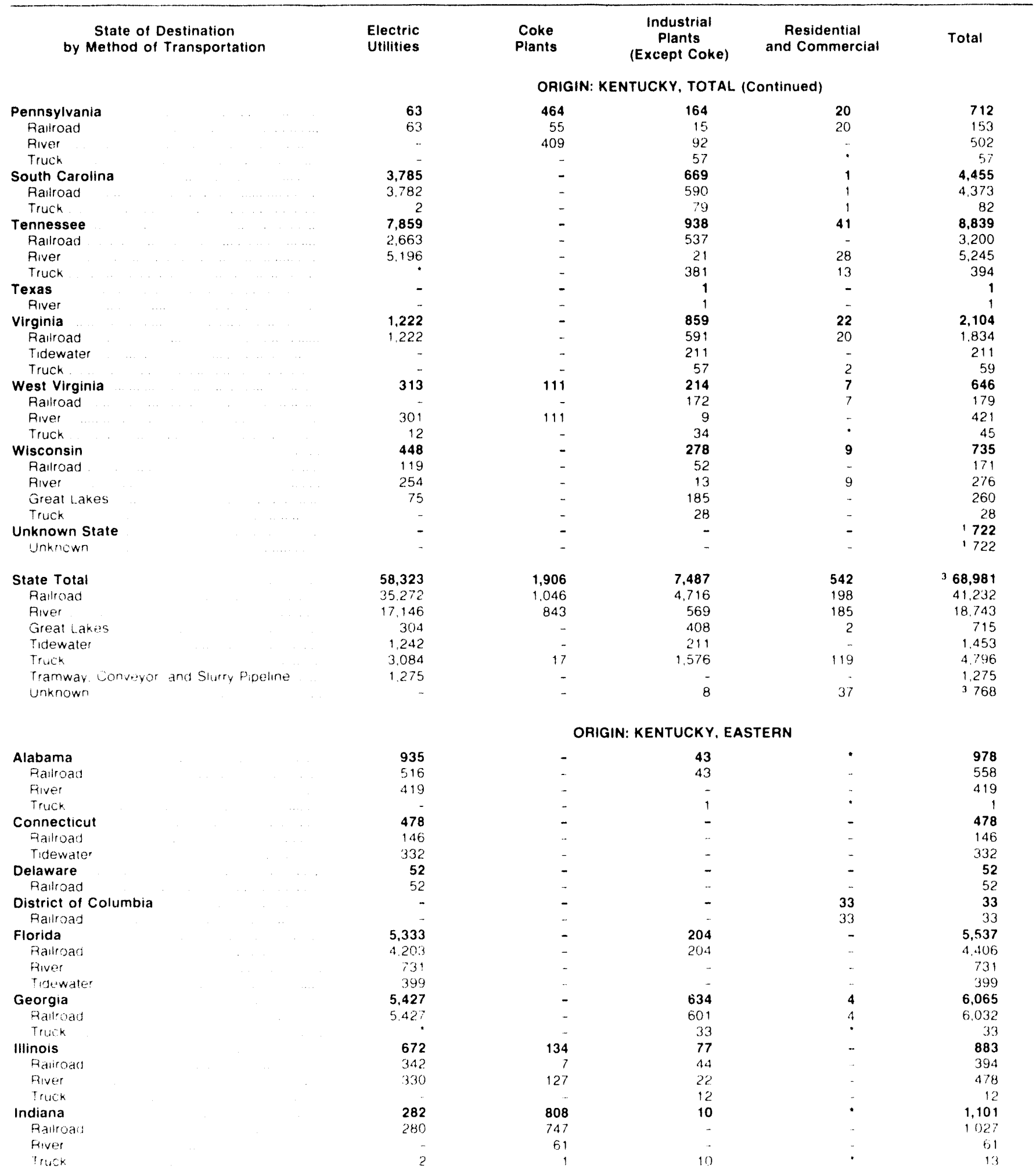


Table 33. Domestic Distribution of U.S. Coal by Origin State, Consumer, Destination and Method of Transportation, January-June 1991 (Continued) (Thousand Short Tons)

\begin{tabular}{|c|c|c|c|c|c|}
\hline $\begin{array}{l}\text { State of Destination } \\
\text { by Method of Transportation }\end{array}$ & $\begin{array}{l}\text { Electric } \\
\text { Uthities }\end{array}$ & $\begin{array}{l}\text { Coke } \\
\text { Plants }\end{array}$ & $\begin{array}{c}\text { Industrial } \\
\text { Plants } \\
\text { (Except Coke) }\end{array}$ & $\begin{array}{c}\text { Residential } \\
\text { and Commercial }\end{array}$ & Total \\
\hline & \multicolumn{5}{|c|}{ ORIGIN: KENTUCKY, EASTERN (Continued) } \\
\hline (1) & - & - & 55 & 97 & 151 \\
\hline 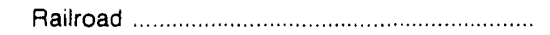 & - & - & 1 & - & 1 \\
\hline 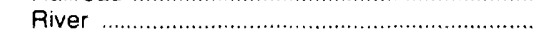 & - & - & 54 & 97 & 150 \\
\hline 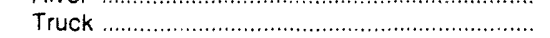 & - & - & ? & - & . \\
\hline Kentucky & 3,003 & 50 & 574 & 90 & 23,717 \\
\hline 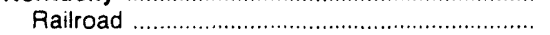 & 818 & 46 & 247 & 18 & 1,129 \\
\hline 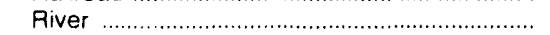 & 757 & - & 2 & - & 759 \\
\hline 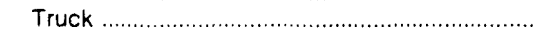 & 1,427 & 4 & 318 & 36 & 1,784 \\
\hline Unknown …1...... & - & - & 8 & 36 & 245 \\
\hline 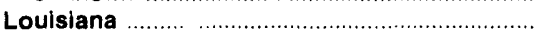 & 102 & - & 50 & - & 152 \\
\hline 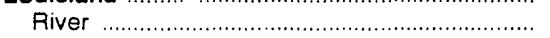 & 97 & - & 50 & - & 147 \\
\hline 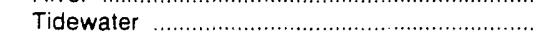 & 4 & - & - & - & 4 \\
\hline 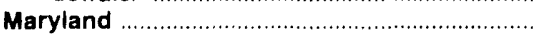 & 194 & 78 & 87 & - & 359 \\
\hline 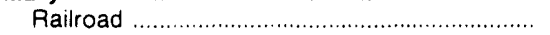 & 51 & - & 87 & - & 138 \\
\hline 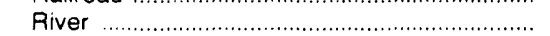 & - & 78 & - & - & 78 \\
\hline Tidewater & 143 & - & - & - & 143 \\
\hline Massachusetts ………………………... & 232 & - & 7 & • & 239 \\
\hline 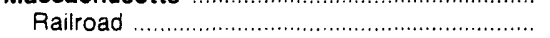 & 15 & - & 4 & . & 20 \\
\hline 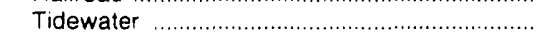 & 217 & - & - & - & 217 \\
\hline 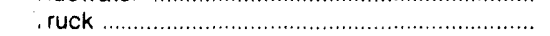 & - & - & 2 & _ & 2 \\
\hline 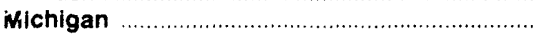 & 3,293 & 4 & 545 & 73 & 3,915 \\
\hline 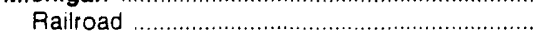 & 3,144 & - & 293 & 72 & 3.509 \\
\hline 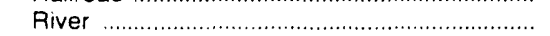 & - & - & 8 & - & 8 \\
\hline 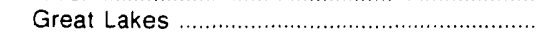 & 149 & - & 217 & 1 & 367 \\
\hline Truck & - & 4 & 27 & : & 31 \\
\hline Minnesota & 29 & - & 2 & - & 31 \\
\hline 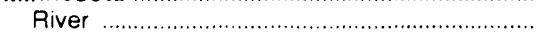 & 9 & - & 2 & - & 11 \\
\hline 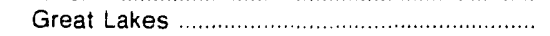 & 20 & - & - & - & 20 \\
\hline 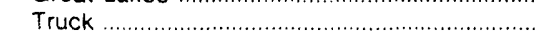 & - & - & . & - & . \\
\hline 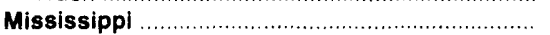 & 1,051 & - & - & - & 1,051 \\
\hline 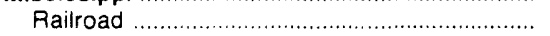 & 1,051 & - & - & - & 1,051 \\
\hline Missouri …………1. & 31 & - & 60 & - & 91 \\
\hline 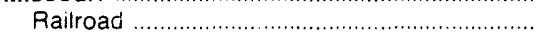 & 31 & - & 2 & - & 33 \\
\hline 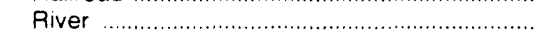 & - & - & 51 & - & 51 \\
\hline 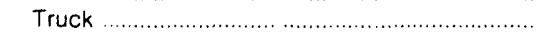 & - & - & 8 & - & 8 \\
\hline New Jersey & 17 & - & 12 & - & 29 \\
\hline 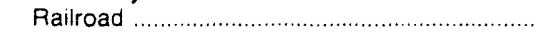 & 17 & - & 12 & - & 29 \\
\hline New York & 768 & 4 & 12 & 17 & 801 \\
\hline 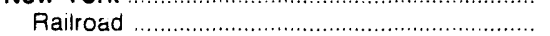 & 768 & 4 & 11 & 7 & 790 \\
\hline River & - & - & - & 9 & 9 \\
\hline 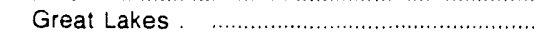 & - & - & - & 1 & 1 \\
\hline 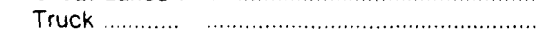 & - & - & . & - & : \\
\hline North Carolina & 4,754 & - & 778 & 14 & 5,546 \\
\hline 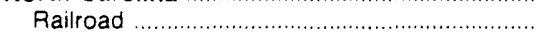 & 4,754 & - & 624 & 12 & 5,390 \\
\hline 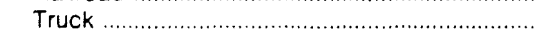 & - & - & 155 & 2 & 157 \\
\hline North Dakota & - & - & 10 & - & 10 \\
\hline 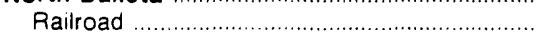 & - & _- & 10 & - & 10 \\
\hline Ohio & 4,155 & 252 & 929 & 60 & 5,396 \\
\hline 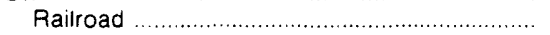 & 1,644 & 187 & 570 & 5 & 2,405 \\
\hline 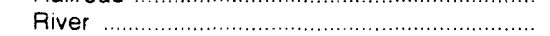 & 2,437 & 56 & 14 & . & 2,507 \\
\hline 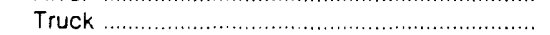 & 75 & 8 & 345 & 55 & 484 \\
\hline 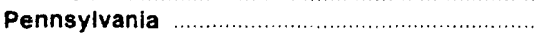 & 63 & 464 & 164 & 20 & 712 \\
\hline Railroad ……1. & 63 & 55 & 15 & 20 & 153 \\
\hline 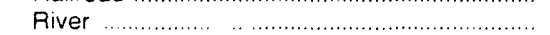 & - & 409 & 92 & - & 502 \\
\hline 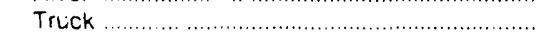 & - & - & 57 & . & 57 \\
\hline South Carolina & 3,785 & - & 669 & 1 & 4,455 \\
\hline Railroad … … & 3,782 & - & 590 & 1 & 4.373 \\
\hline Truck & 2 & - & 79 & 1 & 82 \\
\hline Tennessee & 2,576 & - & 749 & 37 & 3,362 \\
\hline 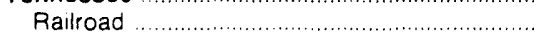 & 2,087 & - & 537 & - & 2,623 \\
\hline River & 490 & - & 21 & 28 & 539 \\
\hline 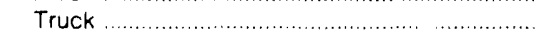 & • & - & 191 & 8 & 200 \\
\hline 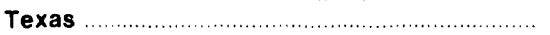 & - & - & 1 & - & 1 \\
\hline${ }_{1}$ & - & - & 1 & - & 1 \\
\hline Virginia & 1,222 & - & 859 & 22 & 2,104 \\
\hline 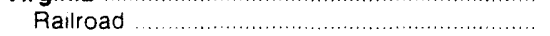 & 1,222 & - & 591 & 20 & 1,834 \\
\hline Tideweater ……… & - & - & 211 & - & 211 \\
\hline Truck ……1. & - & - & 57 & 2 & 59 \\
\hline
\end{tabular}

See footnotes at end of table 
Table 33. Domestic Distribution of U.S. Coal by Origin State, Consumer, Destination and Method of Transportation, January-June 1991 (Continued) (Thousand Short Tons)

\begin{tabular}{|c|c|c|c|c|c|}
\hline $\begin{array}{l}\text { State of Destination } \\
\text { by Method of Transportation }\end{array}$ & $\begin{array}{l}\text { Electric } \\
\text { Utilities }\end{array}$ & $\begin{array}{l}\text { Coke } \\
\text { Plants }\end{array}$ & $\begin{array}{c}\text { Industrial } \\
\text { Plants } \\
\text { (Except Coke) }\end{array}$ & $\begin{array}{c}\text { Residential } \\
\text { and Commercial }\end{array}$ & Total \\
\hline & \multicolumn{5}{|c|}{ ORIGIN: KENTUCKY, EASTERN (Continued) } \\
\hline West Virginia & 294 & 111 & 214 & 7 & 627 \\
\hline 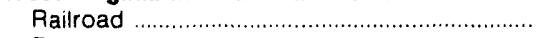 & - & - & 172 & 7 & 179 \\
\hline 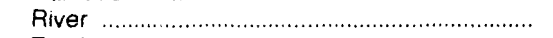 & 282 & 111 & 9 & - & 402 \\
\hline 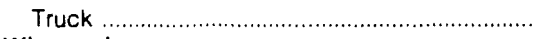 & 12 & - & 33 & * & 45 \\
\hline 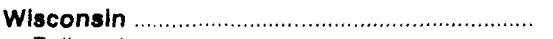 & 230 & - & 234 & 9 & 473 \\
\hline 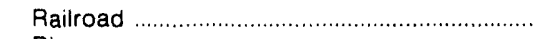 & 119 & - & 10 & - & 129 \\
\hline 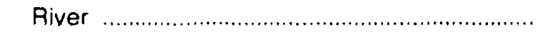 & 42 & - & 13 & 9 & 64 \\
\hline 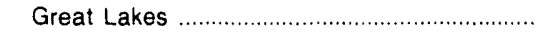 & 69 & - & 185 & - & 254 \\
\hline 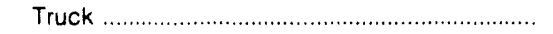 & - & - & 26 & - & 26 \\
\hline Unknown State & - & - & - & - & 1671 \\
\hline 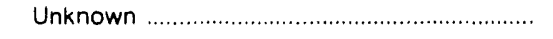 & - & - & - & - & 1671 \\
\hline Statt Total & 38,977 & 1,906 & 6,978 & 484 & ${ }^{3} 49,017$ \\
\hline 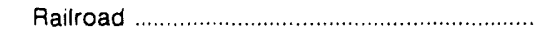 & 30,532 & 1,046 & 4,666 & 198 & 36,442 \\
\hline 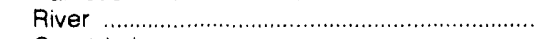 & 5,595 & 843 & 336 & 144 & 6,917 \\
\hline 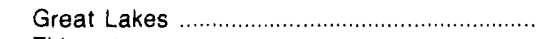 & 238 & - & 402 & 2 & 642 \\
\hline 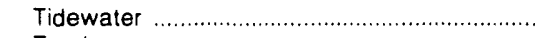 & 1,095 & - & 211 & - & 1,306 \\
\hline 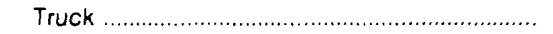 & 1,518 & 17 & 1,355 & 105 & 2,994 \\
\hline \multirow{2}{*}{ 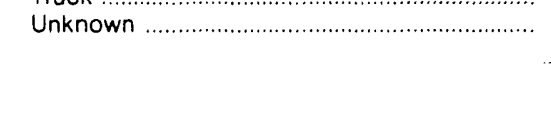 } & - & - & 8 & 36 & 3715 \\
\hline & \multicolumn{5}{|c|}{ ORIGIN: KENTUCKY, WESTERN } \\
\hline Alabama & 982 & - & - & - & 982 \\
\hline 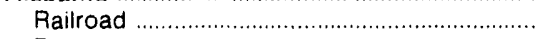 & 159 & - & - & - & 159 \\
\hline 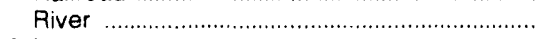 & 823 & - & - & - & 823 \\
\hline Arkansas & - & - & 5 & - & 5 \\
\hline Truck … & - & - & 5 & - & 5 \\
\hline 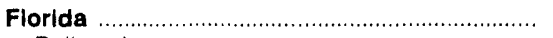 & 1,861 & - & - & - & 1,861 \\
\hline Railroad & 363 & - & - & - & 363 \\
\hline 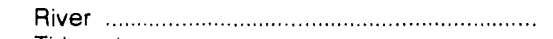 & 1,350 & - & - & - & 1,350 \\
\hline 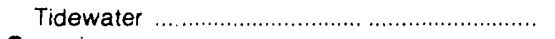 & 147 & - & - & - & 147 \\
\hline Georgia & 668 & - & 8 & - & 676 \\
\hline 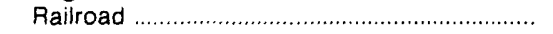 & 668 & - & 8 & - & 676 \\
\hline IIlinols & 142 & - & 125 & - & 267 \\
\hline 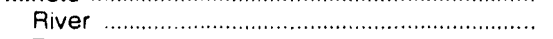 & 126 & - & 122 & - & 248 \\
\hline Truck & 16 & - & 3 & - & 19 \\
\hline Indiana & 1,707 & - & - & - & 1,707 \\
\hline 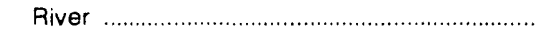 & 1,707 & - & - & - & 1,707 \\
\hline lowa & 36 & - & 84 & 41 & 162 \\
\hline 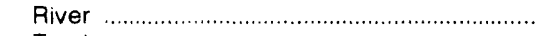 & 36 & - & 84 & 41 & 162 \\
\hline 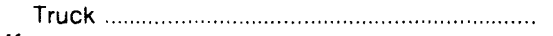 & - & - & * & - & * \\
\hline Kansas & - & - & 3 & - & 3 \\
\hline 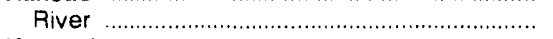 & - & - & 3 & - & 3 \\
\hline 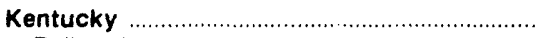 & 7,593 & - & 21 & 11 & 7,626 \\
\hline 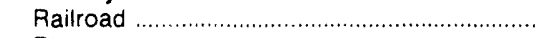 & 2,972 & - & - & 1 & 2,973 \\
\hline 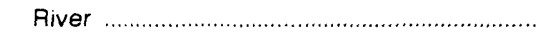 & 1,796 & - & - & $\cdot$ & 1,797 \\
\hline 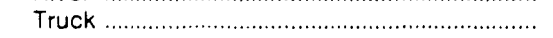 & 1,550 & - & 21 & 10 & 1,581 \\
\hline Tramway, Conveyor, and Slurry Pipeline ....... & 1,275 & - & - & - & 1,275 \\
\hline 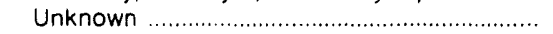 & - & - & - & 1 & 1 \\
\hline 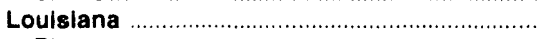 & 217 & - & - & - & 217 \\
\hline 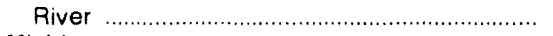 & 217 & - & - & - & 217 \\
\hline 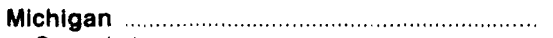 & 61 & - & 6 & - & 67 \\
\hline Great Lakes & 61 & - & 6 & - & 67 \\
\hline 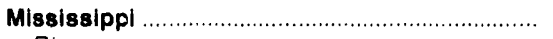 & 42 & - & 23 & - & 65 \\
\hline 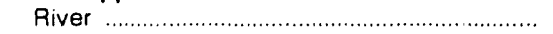 & 42 & - & 23 & - & 65 \\
\hline 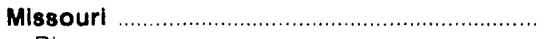 & 293 & - & - & * & 293 \\
\hline 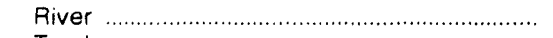 & 293 & - & - & - & 293 \\
\hline Truck & - & - & - & * & * \\
\hline Ohlo & 223 & - & - & - & 223 \\
\hline 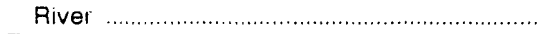 & 223 & - & - & - & 223 \\
\hline Tennessee & 5,283 & - & 189 & 5 & 5,477 \\
\hline 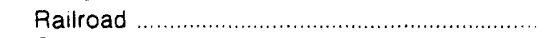 & 577 & - & - & - & 577 \\
\hline 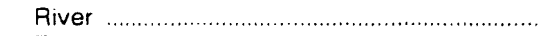 & 4,706 & - & - & - & 4,706 \\
\hline Truck & - & - & 189 & 5 & 194 \\
\hline West Virginia & 19 & - & * & - & 19 \\
\hline 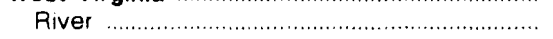 & 19 & - & - & - & 19 \\
\hline Truck & - & - & $\cdot$ & - & * \\
\hline
\end{tabular}

See footnotes at end of table. 
Table 33. Domestic Distribution of U.S. Coal by Origin State, Consumer, Destination and Method of Transportation, January-June 1991 (Continued)

(Thousand Short Tons)

\begin{tabular}{|c|c|c|c|c|c|}
\hline $\begin{array}{l}\text { State of Destination } \\
\text { by Method of Transportation }\end{array}$ & $\begin{array}{l}\text { Electric } \\
\text { Utilities }\end{array}$ & $\begin{array}{l}\text { Coke } \\
\text { Plants }\end{array}$ & $\begin{array}{c}\text { Industrial } \\
\text { Plants } \\
\text { (Except Coke) }\end{array}$ & $\begin{array}{l}\text { Residential } \\
\text { and Commercial }\end{array}$ & Total \\
\hline & \multicolumn{5}{|c|}{ ORIGIN: KENTUCKY, WESTERN (Continued) } \\
\hline Wisconsin & 218 & - & 44 & - & 262 \\
\hline Railroad & - & - & 42 & - & 42 \\
\hline River & 212 & - & - & - & 212 \\
\hline Great Lakes & 6 & - & - & - & 6 \\
\hline Truck & - & - & 2 & - & 2 \\
\hline Unknown State. & - & - & - & - & 52 \\
\hline Unknown ....... & - & - & - & - & 152 \\
\hline State Total & 19,346 & - & 509 & 57 & 19,904 \\
\hline Ralload ‥1.1.1. & 4.739 & - & 49 & 1 & 4.789 \\
\hline River & 11.552 & - & 232 & 42 & 11,826 \\
\hline 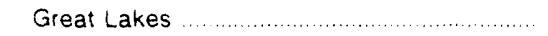 & 67 & - & 6 & - & 73 \\
\hline Tidewater & 147 & - & - & - & 147 \\
\hline 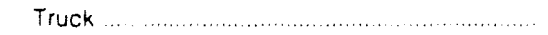 & 1.566 & - & 221 & 14 & 1,802 \\
\hline Tramway. Conveyor, and Slurry Pipeline ...... & 1.275 & - & - & - & 1,275 \\
\hline \multirow[t]{2}{*}{ 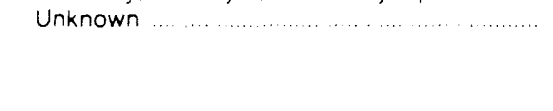 } & - & - & - & 1 & 152 \\
\hline & \multicolumn{5}{|c|}{ ORIGIN: LOUISIANA } \\
\hline Loulsiana & 1,287 & - & - & - & 1,287 \\
\hline Truck & 177 & - & - & - & 177 \\
\hline Tramway. Conveyor. and Slurry Pipeline & 1.111 & - & - & - & 1.111 \\
\hline State Total & $\uparrow, 287$ & - & - & - & 1,287 \\
\hline Truck ..... & 177 & - & - & - & 177 \\
\hline \multirow[t]{2}{*}{ Tramway, Conveyor, and Slurry Pipeline. } & 1.111 & - & - & - & 1,111 \\
\hline & \multicolumn{5}{|c|}{ ORIGIN: MARYLAND } \\
\hline Maryland & 426 & - & 184 & 1 & 611 \\
\hline Ralload & 401 & - & 125 & - & 526 \\
\hline Truck & 25 & - & 48 & 1 & 74 \\
\hline … & - & - & 12 & • & 12 \\
\hline New York & 6 & - & - & - & 6 \\
\hline Raılroad & 3 & - & - & - & 3 \\
\hline Truck & 3 & - & - & - & 3 \\
\hline Pennsylvania & 13 & - & - & * & 13 \\
\hline Truck & 13 & - & - & • & 13 \\
\hline Virginia $\ldots \ldots \ldots \ldots$ & 25 & - & - & - & 25 \\
\hline Raltroad & 12 & - & - & - & 12 \\
\hline Truck .................... & 13 & - & - & - & 13 \\
\hline West Virginia & 994 & - & - & - & 994 \\
\hline$\ldots \ldots \ldots$ & 682 & - & - & - & 682 \\
\hline$\ldots \ldots \ldots \ldots \ldots$ & 313 & - & - & " & 313 \\
\hline Unknown State & - & - & - & - & 16 \\
\hline Unknown & - & - & - & - & 16 \\
\hline State Total $\ldots . . .$. & 1,464 & - & 184 & 2 & 1,655 \\
\hline Railroad & 1.097 & - & 125 & - & 1.222 \\
\hline Truck & 367 & - & 48 & 2 & 416 \\
\hline Unknown & - & - & 12 & . & 18 \\
\hline
\end{tabular}

ORIGIN: MISSOURI
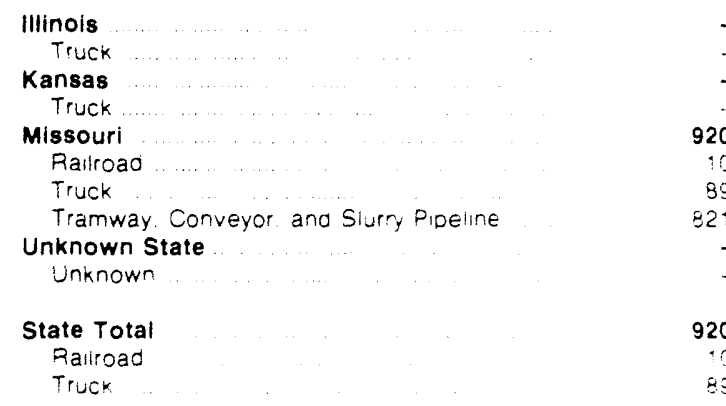

See tcoinotes ai end of tabie

$\begin{array}{rrr}: & - & \\ 3 & - & \\ 3 & - & 3 \\ 5 & - & 3 \\ - & 67 & 992 \\ 5 & - & 10 \\ - & 67 & 167 \\ - & - & 821 \\ - & - & 12 \\ 8 & - & 12 \\ - & 67 & 1997 \\ 8 & - & 10 \\ & 67 & 164\end{array}$



Table 33. Domestic Distribution of U.S. Coal by Origin State, Consumer, Destination
and Method of Transportation, January-June 1991 (Continued)

(Thousand Short Tons)

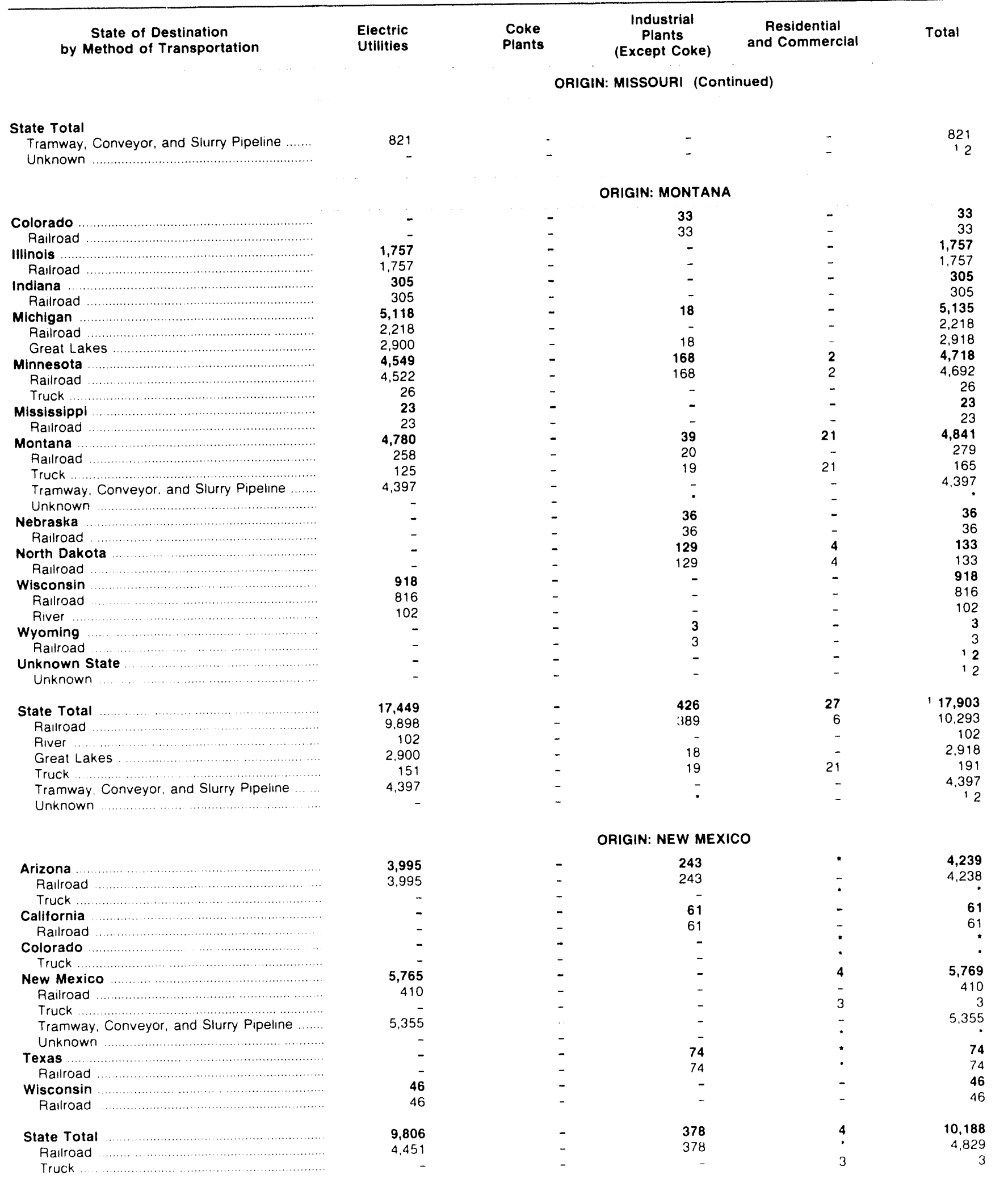

See footnotes at end of table 
Table 33. Domestic Distribution of U.S. Coal by Origin State, Consumer, Destination and Method of Transportation, January-June 1991 (Continued) (Thousand Short Tons)

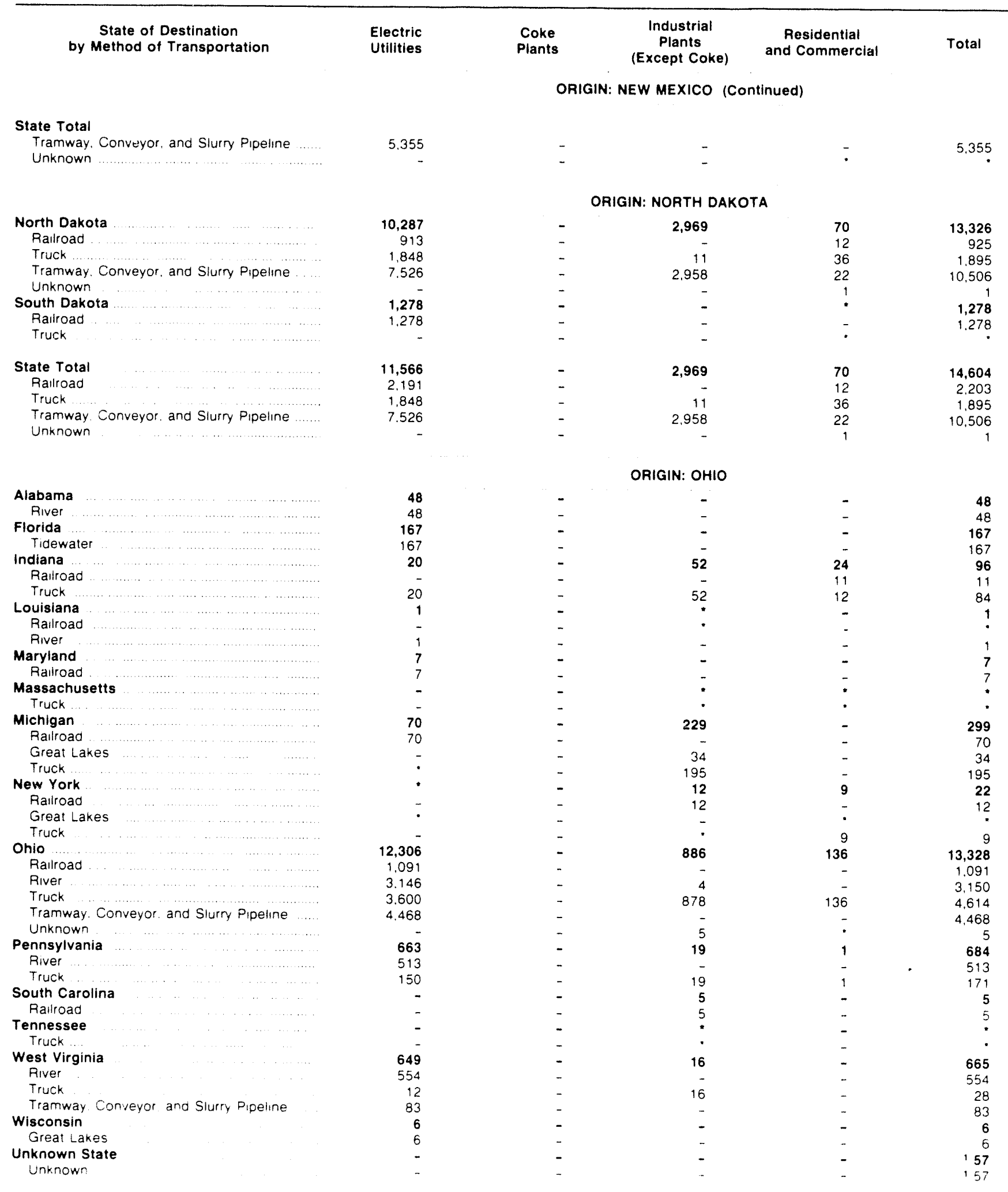

See tooinotes at end tabie 
Table 33. Domestic Distribution of U.S. Coal by Origin State, Consumer, Destination and Method of Transportation, January-June 1991 (Continued) (Thousand Short Tons)

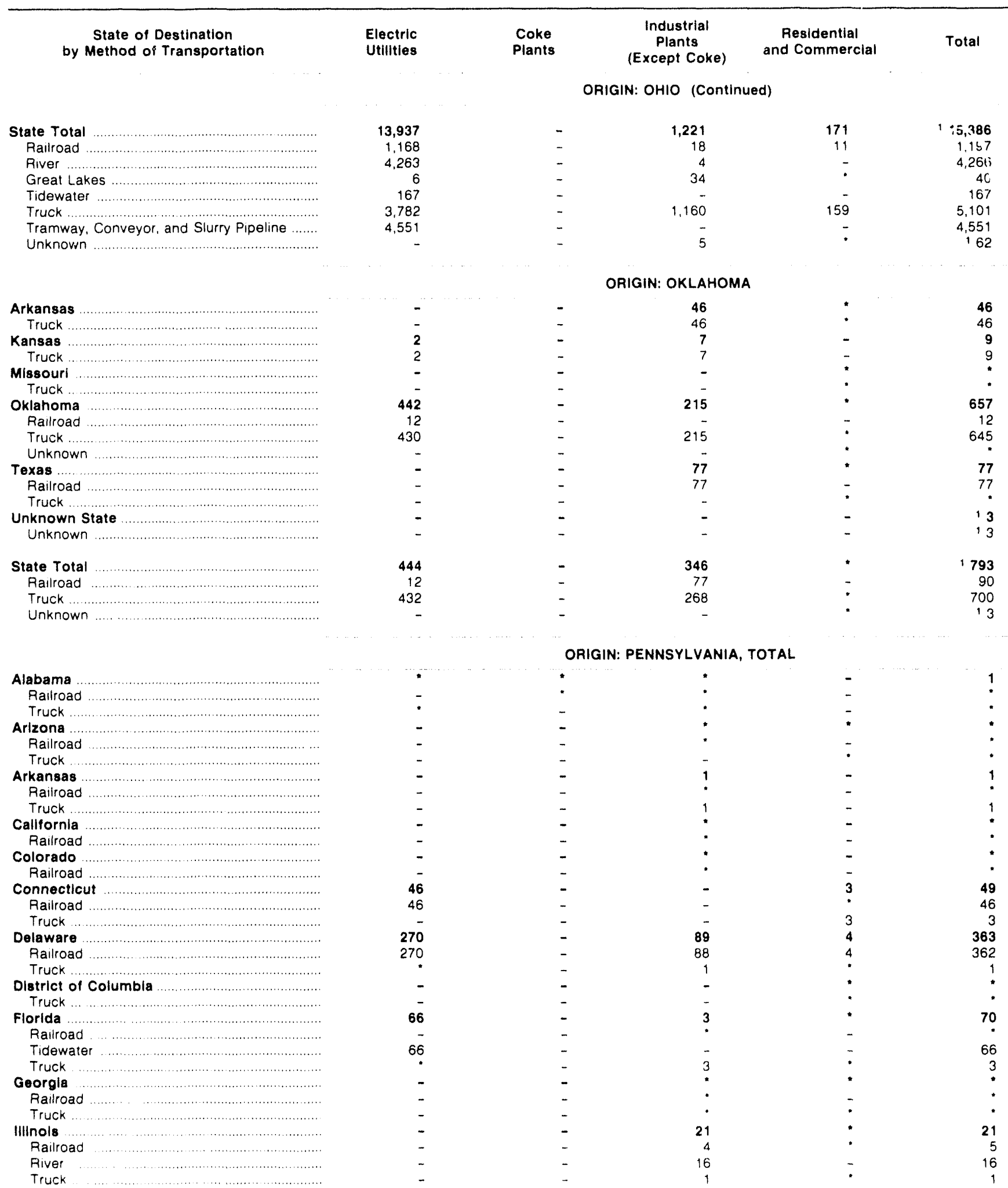

See footnotes at end of table. 
Table 33. Domestic Distribution of U.S. Coal by Origin State, Consumer, Destination and Method of Transportation, January-June 1991 (Continued)

(Thousand Short Tons)

\begin{tabular}{|c|c|c|c|c|c|}
\hline $\begin{array}{l}\text { State of Destination } \\
\text { by Method of Transportation }\end{array}$ & $\begin{array}{l}\text { Electric } \\
\text { Utilities }\end{array}$ & $\begin{array}{l}\text { Coke } \\
\text { Plants }\end{array}$ & $\begin{array}{c}\text { Industrial } \\
\text { Plants } \\
\text { (Except Coke) }\end{array}$ & $\begin{array}{l}\text { Residential } \\
\text { and Commercial }\end{array}$ & Total \\
\hline & \multicolumn{5}{|c|}{ ORIGIN: PENNSYLVANIA, TOTAL (Continued) } \\
\hline Indlana & - & 161 & 11 & 1 & 173 \\
\hline 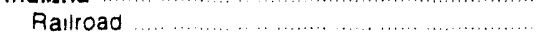 & - & 161 & $\because$ & - & 161 \\
\hline River & - & - & 11 & - & 11 \\
\hline Truck & - & - & $\cdot$ & 1 & 1 \\
\hline lowa & - & - & 83 & • & 83 \\
\hline - & - & - & 83 & $\cdot$ & 83 \\
\hline … & - & - & • & - & - \\
\hline 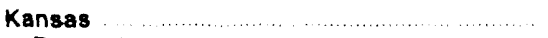 & - & - & • & - & • \\
\hline Raitroad & - & - & - & - & • \\
\hline Truck & - & - & • & - & . \\
\hline Kentucky & - & - & 11 & $\cdot$ & 11 \\
\hline Raliroad & - & - & 11 & - & 11 \\
\hline Truck & - & - & - & • & $\cdot$ \\
\hline Loulisiana & - & - & - & - & • \\
\hline Raliroad & - & - & • & - & • \\
\hline 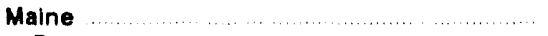 & - & - & - & 3 & 3 \\
\hline 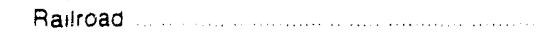 & - & - & - & 1 & 1 \\
\hline (1) & - & - & - & 2 & 2 \\
\hline$\ldots \ldots \ldots \ldots \ldots \ldots \ldots \ldots \ldots$ & 1,139 & 56 & 78 & 2 & 1,275 \\
\hline Rallroad & 1.072 & 56 & 30 & • & 1.158 \\
\hline 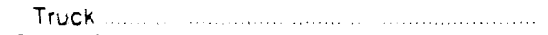 & 67 & - & 48 & 2 & 116 \\
\hline Massachusetts & 69 & - & 2 & 8 & 79 \\
\hline Rallroad ... & 68 & - & 1 & 2 & 70 \\
\hline 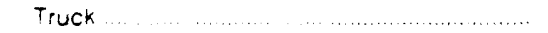 & 1 & - & 2 & 6 & 9 \\
\hline ㄷ... & 614 & 35 & 42 & 12 & 704 \\
\hline 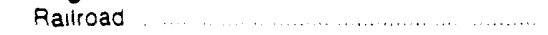 & 456 & 7 & 21 & - & 484 \\
\hline Great Lakes & 158 & 28 & 21 & - & 207 \\
\hline Truck & - & - & 1 & 12 & 13 \\
\hline Minnesota & 329 & - & 19 & • & 348 \\
\hline … & 325 & - & • & - & 325 \\
\hline Great Lakes & - & - & 18 & - & 18 \\
\hline Truck & 5 & - & • & - & 5 \\
\hline Mississippl $\ldots$ & - & - & - & - & • \\
\hline Raulroad & - & - & - & - & • \\
\hline New Hampshire & 394 & - & 1 & 6 & 401 \\
\hline Rauroad & 394 & - & 1 & 2 & 397 \\
\hline Truck & - & - & - & 5 & 5 \\
\hline New Jersey & 8 & - & 4 & 4 & 16 \\
\hline Rallroad & 8 & - & • & : & 8 \\
\hline$(2, \ldots \ldots \ldots+\ldots$ & - & - & 4 & 4 & 8 \\
\hline 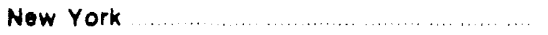 & 2,643 & 409 & 243 & 92 & 3,387 \\
\hline Railroad & 2.101 & 403 & 111 & 15 & 2,630 \\
\hline River & 228 & - & 13 & - & 241 \\
\hline Truck & 313 & 6 & 120 & 77 & 516 \\
\hline North Carolina & - & - & • & - & - \\
\hline Raulioad $\ldots$ & - & - & • & - & . \\
\hline North Dakota & - & - & - & - & . \\
\hline Ralload & - & - & . & - & . \\
\hline Truck & - & - & - & • & $\cdot$ \\
\hline Ohlo $\ldots \ldots$ & 1,644 & - & 126 & - & 1,770 \\
\hline Rallroad & 272 & - & 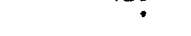 & - & 272 \\
\hline River $\ldots \ldots \ldots \ldots$ & 1,126 & - & - & - & 1.126 \\
\hline Great Lakes & 12 & - & 36 & - & 48 \\
\hline Truck & 234 & - & 90 & - & 324 \\
\hline 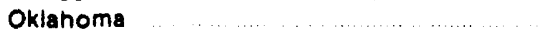 & - & - & - & - & . \\
\hline . $\ldots \ldots$ & - & - & - & - & . \\
\hline … & - & - & - & - & . \\
\hline$\ldots \ldots \ldots$ & - & - & - & - & . \\
\hline $\begin{array}{ccccc}\ldots \ldots & \ldots & \ldots\end{array}$ & 15,460 & 1,022 & 1,144 & 646 & 18,273 \\
\hline$\ldots \ldots$ & 5.939 & 261 & 47 & 18 & 6.265 \\
\hline River ..... & 532 & 709 & 7 & - & 1.249 \\
\hline Great Lakıs & 22 & - & - & - & 22 \\
\hline Tidewater & 34 & - & - & - & 36 \\
\hline Truck ... & 3,994 & 52 & 1.064 & 625 & 5.736 \\
\hline Tramway Conveyor and Siurry Pipeline & 4.939 & - & - & - & 4,939 \\
\hline Unknown & - & - & 26 & 3 & 29 \\
\hline
\end{tabular}

See foomoles a: end of tabie 
Table 33. Domestic Distribution of U.S. Coal by Origin State, Consumer, Destination and Method of Transportation, January-June 1991 (Continued) (Thousand Short Tons)

\begin{tabular}{|c|c|c|c|c|c|}
\hline $\begin{array}{l}\text { State of Destination } \\
\text { by Method of Transportation }\end{array}$ & $\begin{array}{l}\text { Electric } \\
\text { Utilities }\end{array}$ & $\begin{array}{l}\text { Coke } \\
\text { Plants }\end{array}$ & $\begin{array}{c}\text { Industrial } \\
\text { Plants } \\
\text { (Except Coke) }\end{array}$ & $\begin{array}{c}\text { Residential } \\
\text { and Commercial }\end{array}$ & Total \\
\hline & \multicolumn{5}{|c|}{ ORIGIN: PENNSYLVANIA, TOTAL (Continued) } \\
\hline Rhode Island & - & - & - & 1 & 1 \\
\hline Railroad & - & - & - & : & : \\
\hline Truck & - & - & - & 1 & 1 \\
\hline South Carolina & - & - & 5 & $*$ & 5 \\
\hline 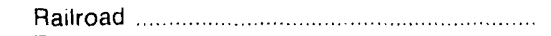 & - & - & $\cdot$ & * & * \\
\hline 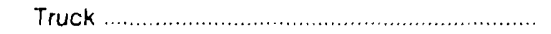 & - & - & 5 & - & 5 \\
\hline South Dakota .............. & - & - & 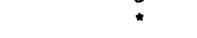 & - & * \\
\hline Railroad & - & - & * & - & * \\
\hline Tennessee & - & - & 3 & 6 & 9 \\
\hline Railroad & - & - & 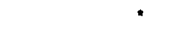 & 6 & 6 \\
\hline Truck & - & - & 3 & - & 3 \\
\hline Texas & - & - & 2 & * & 2 \\
\hline Railroad & - & - & $\overline{1}$ & - & 1 \\
\hline 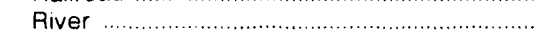 & - & - & 2 & - & 2 \\
\hline 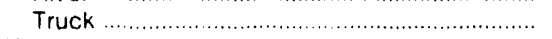 & - & - & 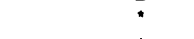 & • & . \\
\hline 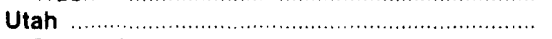 & - & 153 & * & - & 153 \\
\hline Railroad & - & 153 & * & - & 153 \\
\hline 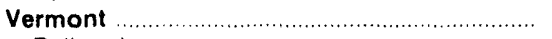 & - & - & * & 2 & 2 \\
\hline 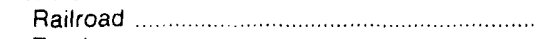 & - & - & - & $\cdot$ & $*$ \\
\hline 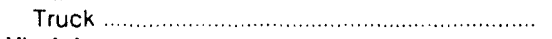 & - & - & $\cdot$ & 2 & 2 \\
\hline Virginia & 43 & - & 7 & * & 50 \\
\hline Railroad & 32 & - & • & - & 32 \\
\hline 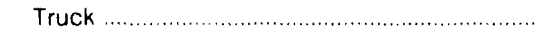 & 11 & - & 7 & * & 18 \\
\hline West Virginia & 499 & - & 276 & * & 775 \\
\hline 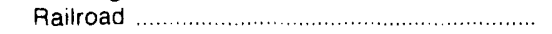 & 13 & - & * & - & 13 \\
\hline 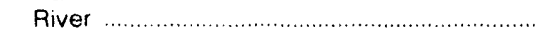 & 403 & - & 146 & - & 549 \\
\hline 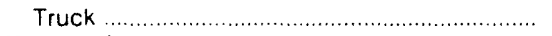 & 83 & - & 130 & * & 213 \\
\hline 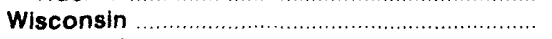 & 726 & - & 26 & * & 753 \\
\hline 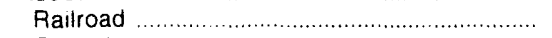 & 726 & - & 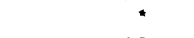 & - & 727 \\
\hline Great Lakes & - & - & 26 & - & 26 \\
\hline 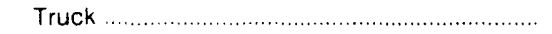 & - & - & * & * & \\
\hline Wyoming & - & - & * & * & * \\
\hline Railroad & - & - & * & * & * \\
\hline Unknown State & - & - & - & - & 1170 \\
\hline 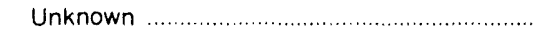 & - & - & - & - & 1170 \\
\hline State Total & 23,951 & 1,836 & 2,201 & 791 & 128,948 \\
\hline Railroad & 11,721 & 1,041 & 398 & 48 & 13,209 \\
\hline River & 2,290 & 709 & 195 & - & 3,194 \\
\hline 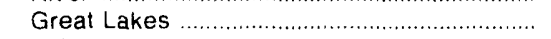 & 192 & 28 & 101 & - & 322 \\
\hline 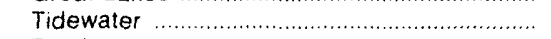 & 100 & - & - & - & 100 \\
\hline Truck & 4,709 & 58 & 1,480 & 740 & 6,986 \\
\hline Tramway, Conveyor, and Slurry Pipeline ....... & 4.939 & - & - & - & 4,939 \\
\hline 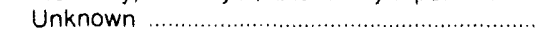 & - & - & 26 & 3 & 1198 \\
\hline
\end{tabular}

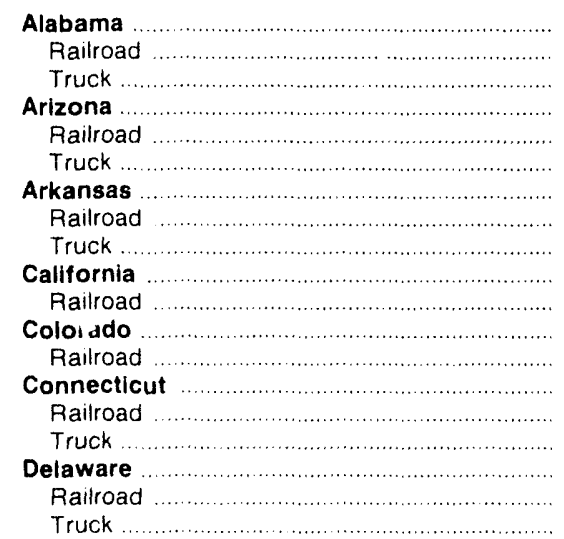

ORIGIN: PENNSYLVANIA, ANTHRACITE

See footnotes at end of table. 
Table 33. Domestic Distribution of U.S. Coal by Origin State, Consumer, Destination and Method of Transportation, January-June 1991 (Continued) (Thousand Short Tons)

\begin{tabular}{|c|c|c|c|c|c|}
\hline $\begin{array}{l}\text { State of Destination } \\
\text { by Method of Transportation }\end{array}$ & $\begin{array}{l}\text { Electric } \\
\text { Utilities }\end{array}$ & $\begin{array}{l}\text { Coke } \\
\text { Plants }\end{array}$ & $\begin{array}{c}\text { Industrial } \\
\text { Plants } \\
\text { (Except Coke) }\end{array}$ & $\begin{array}{l}\text { Residential } \\
\text { and Commercial }\end{array}$ & Total \\
\hline & \multicolumn{5}{|c|}{ ORIGIN: PENNSYLVANIA, ANTHRACITE (Continued) } \\
\hline District of Columbia & - & - & - & • & * \\
\hline 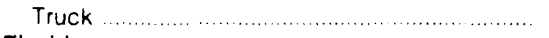 & - & - & - & $\cdot$ & - \\
\hline Florida & * & - & 3 & * & 3 \\
\hline 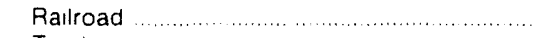 & - & - & $\cdot$ & & $\cdot$ \\
\hline Truck & $\cdot$ & - & 3 & $\cdot$ & 3 \\
\hline Georgia & - & - & - & * & - \\
\hline Railroad & - & - & $\cdot$ & - & - \\
\hline Truck & - & - & $\cdot$ & $\cdot$ & - \\
\hline . & - & - & 5 & - & 5 \\
\hline Railroad ... & - & - & 4 & • & 5 \\
\hline 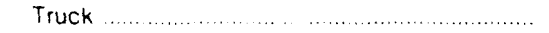 & - & - & 1 & $\cdot$ & 1 \\
\hline Indiana & - & - & $\cdot$ & 1 & 1 \\
\hline Railroad & - & - & • & - & : \\
\hline Truck & - & - & • & 1 & 1 \\
\hline lowa & - & - & 17 & - & 17 \\
\hline Railroad & - & - & 16 & $\cdot$ & 17 \\
\hline Truck & - & - & $\cdot$ & - & - \\
\hline Kansas & - & - & * & - & - \\
\hline Ralroad $\ldots \ldots \ldots \ldots$ & - & - & • & - & - \\
\hline Truck & - & - & • & - & - \\
\hline Kentucky & - & - & 11 & • & 11 \\
\hline 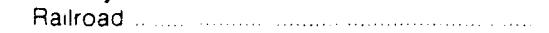 & - & - & 11 & - & 11 \\
\hline Truck & - & - & - & • & $\cdot$ \\
\hline 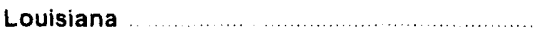 & - & - & • & - & * \\
\hline Rasiroad $\ldots \ldots \ldots$ & - & - & • & - & - \\
\hline Maine $\ldots \ldots \ldots$ & - & - & - & 3 & 3 \\
\hline Railroad & - & - & - & 1 & ; \\
\hline Truck & - & - & - & 2 & 2 \\
\hline Maryland & - & - & 3 & 2 & 4 \\
\hline Railroad & - & - & 3 & $\cdot$ & 3 \\
\hline 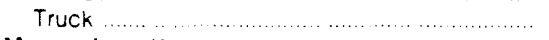 & - & - & $\cdot$ & 2 & 2 \\
\hline Massachusetts & - & - & * & 8 & 8 \\
\hline Rasiroad & - & - & $\cdot$ & 2 & 2 \\
\hline Truck & - & - & - & 6 & 6 \\
\hline 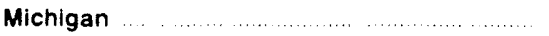 & - & - & 1 & . & 1 \\
\hline Rairoad & - & - & : & - & ? \\
\hline 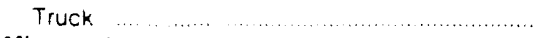 & - & - & 1 & $\cdot$ & 1 \\
\hline Minnesota & - & - & * & - & - \\
\hline 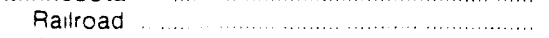 & - & - & - & - & * \\
\hline Truck $\ldots$ & - & - & • & $\cdot$ & - \\
\hline 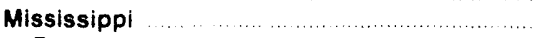 & - & - & * & - & - \\
\hline Ratroad & - & - & - & - & - \\
\hline New Hampshire $\ldots$ & - & - & - & 6 & 6 \\
\hline Ralroad $\ldots \ldots \ldots \ldots \ldots$ & - & - & $\cdot$ & 2 & 2 \\
\hline 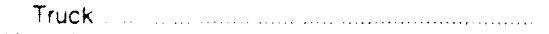 & - & - & - & 4 & 4 \\
\hline New Jersey & - & - & 4 & 4 & 8 \\
\hline (. & - & - & $\cdot$ & $\cdot$ & • \\
\hline Truck $\ldots \ldots \ldots \ldots$ & - & - & 4 & 4 & 8 \\
\hline 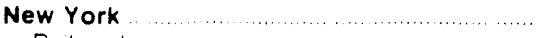 & 1 & - & 7 & 47 & 56 \\
\hline 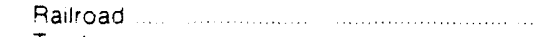 & - & - & $\cdot$ & 8 & 8 \\
\hline Truck & 1 & - & 7 & 39 & 48 \\
\hline North Carolina $\ldots \ldots \ldots \ldots \ldots \ldots$ & - & - & $\cdot$ & - & * \\
\hline 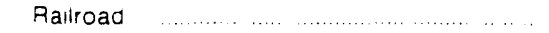 & - & - & • & - & - \\
\hline North Dakota & - & - & * & * & - \\
\hline Railroad $\ldots \ldots \ldots \ldots \ldots \ldots$ & - & - & • & - & - \\
\hline 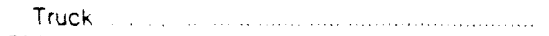 & - & . & - & $\cdot$ & - \\
\hline 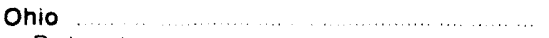 & - & - & 6 & * & 7 \\
\hline 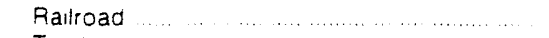 & - & - & $\cdot$ & • & - \\
\hline Truck ... . . . . & - & - & 6 & • & 6 \\
\hline Oklahoma $\ldots \ldots \ldots \ldots$ & - & - & . & - & * \\
\hline 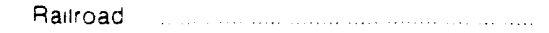 & .- & - & $\cdot$ & - & • \\
\hline Oregon & - & - & * & - & * \\
\hline Ralload $\ldots \ldots \ldots$ & - & - & • & - & - \\
\hline Pennsylvania $\ldots \ldots \ldots \ldots$ & 489 & - & 215 & 342 & 1,046 \\
\hline Raliroad $\ldots \ldots \ldots$ & - & - & 16 & 12 & 28 \\
\hline$\ldots \ldots \ldots \ldots \ldots$ & 295 & - & 198 & 328 & B21 \\
\hline Tramway. Conveyor. and Sturry Pipeline $\ldots . .$. & 194 & - & - & - & 194 \\
\hline Unknown & - & - & 1 & 2 & 2 \\
\hline
\end{tabular}

See tootnotes at end of table 
Table 33. Domestic Distribution of U.S. Coal by Origin State, Consumer, Destination and Method of Transportation, January-June 1991 (Continued)

(Thousand Short Tons)

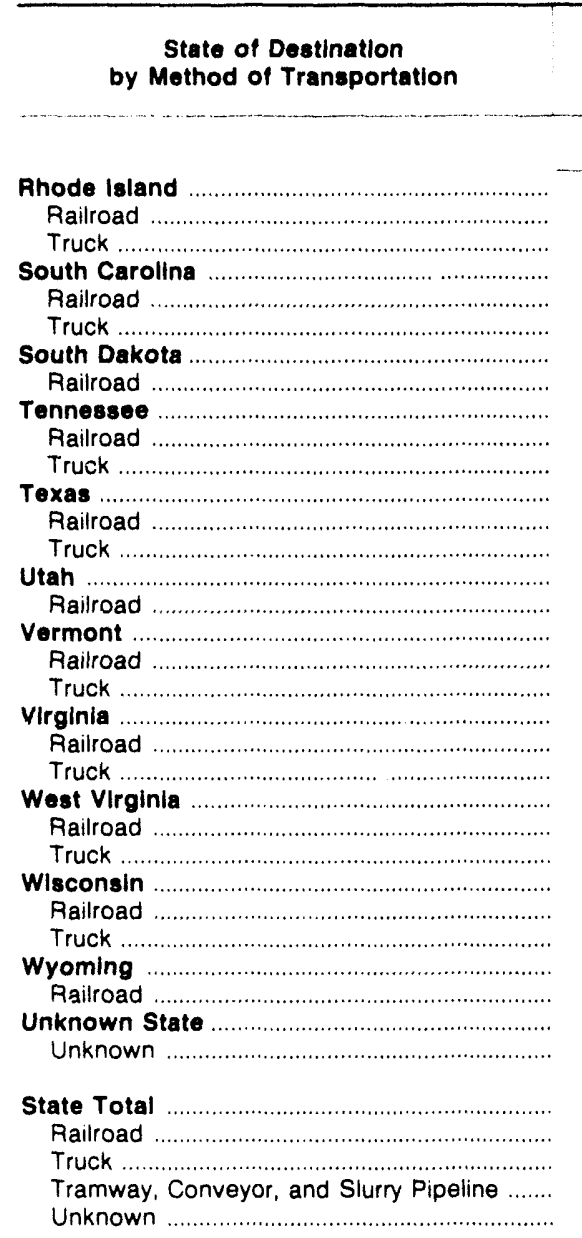

\begin{tabular}{|c|c|c|}
\hline $\begin{array}{l}\text { Electric } \\
\text { Utilities }\end{array}$ & $\begin{array}{l}\text { Coke } \\
\text { Plants }\end{array}$ & $\begin{array}{c}\text { Induetrial } \\
\text { Plante } \\
\text { (Except Coke) }\end{array}$ \\
\hline
\end{tabular}

Residential
and Commerclal Total

ORIGIN: PENNSYLVANIA, ANTHRACITE (Continued)

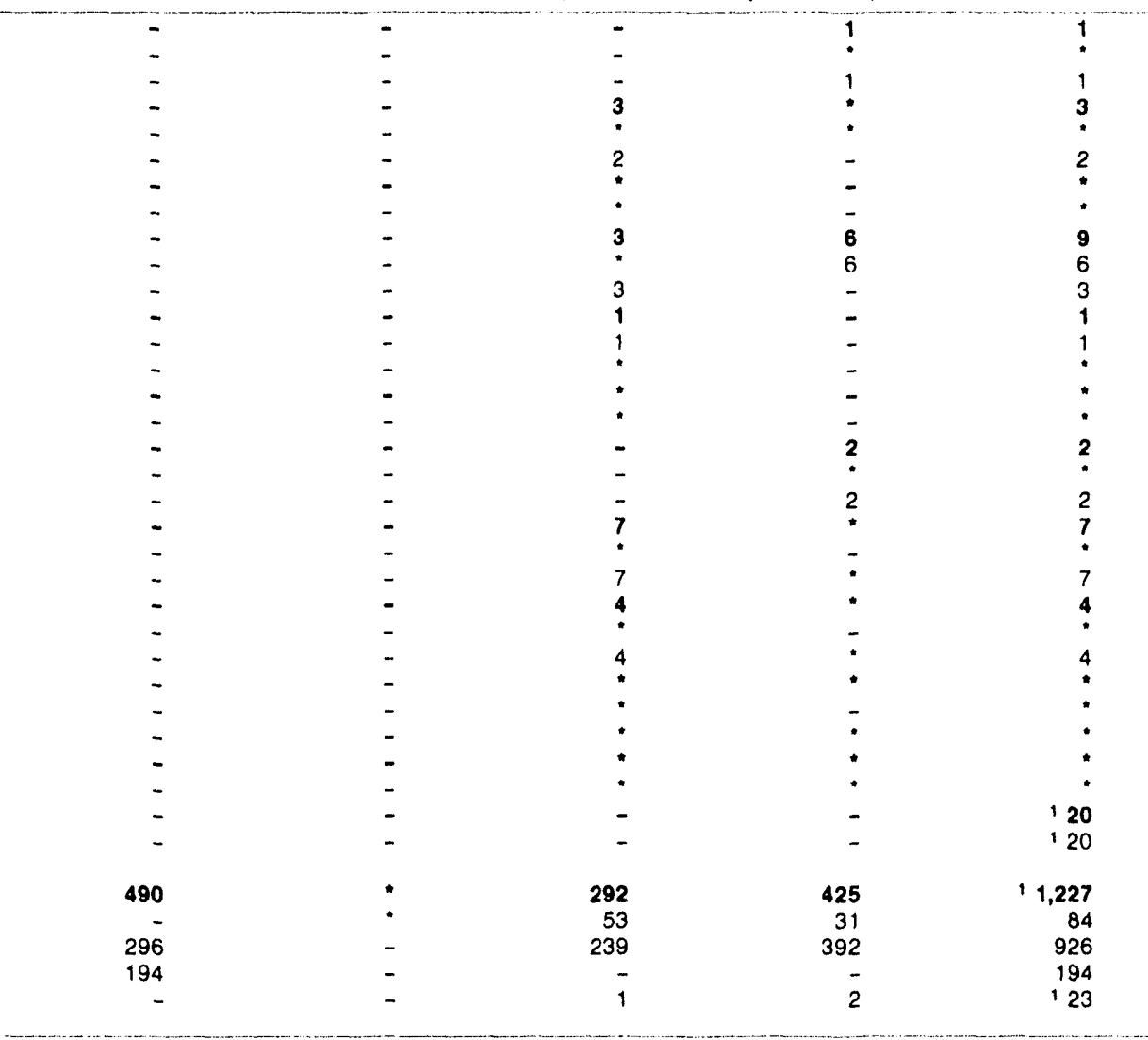

ORIGIN: PENNSYLVANIA, BITUMINOUS

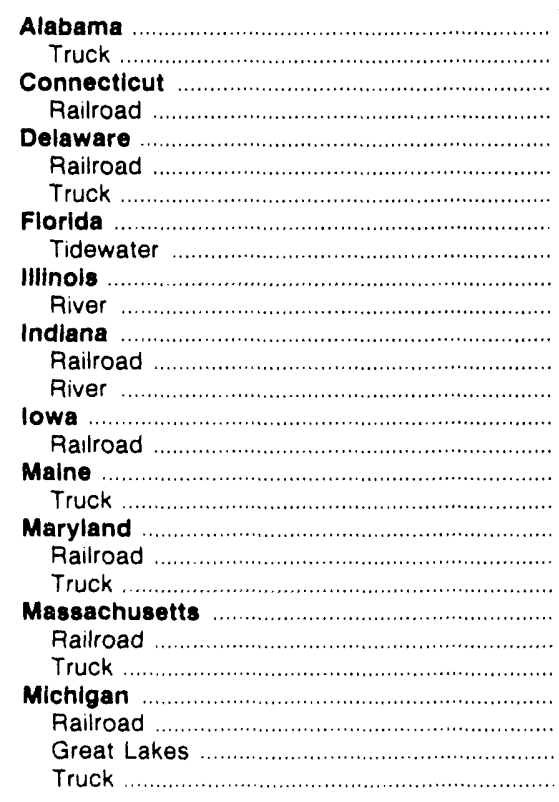

\begin{tabular}{|c|c|c|}
\hline & \multicolumn{2}{|c|}{ ORIGIN: PENNSYLVANIA } \\
\hline 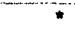 & - & - \\
\hline * & - & - \\
\hline 46 & - & - \\
\hline 46 & - & - \\
\hline 270 & - & 89 \\
\hline 270 & - & 88 \\
\hline 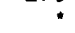 & - & 1 \\
\hline 66 & - & - \\
\hline 66 & - & - \\
\hline - & - & 16 \\
\hline - & - & 16 \\
\hline - & 161 & 11 \\
\hline- & 161 & - \\
\hline - & - & 11 \\
\hline - & - & 67 \\
\hline - & - & 67 \\
\hline - & - & - \\
\hline- & - & - \\
\hline 1,139 & 56 & 75 \\
\hline 1,072 & 56 & 27 \\
\hline 67 & - & 48 \\
\hline 69 & - & 2 \\
\hline 68 & - & $\overline{1}$ \\
\hline 1 & - & 2 \\
\hline 614 & 35 & 42 \\
\hline 456 & 7 & 21 \\
\hline 158 & 28 & 21 \\
\hline .. & - & . \\
\hline
\end{tabular}

\begin{tabular}{rr} 
& \\
\hline- & $*$ \\
- & 46 \\
- & 46 \\
4 & 363 \\
4 & 362 \\
- & 1 \\
- & 66 \\
- & 66 \\
- & 16 \\
- & 16 \\
- & 173 \\
- & 161 \\
- & 11 \\
- & 67 \\
- & 67 \\
$:$ & $*$ \\
$*$ & 1,270 \\
- & 1,156 \\
- & 115 \\
- & 71 \\
- & 68 \\
\hline- & 3 \\
- & 704 \\
\hline- & 484 \\
12 & 207 \\
\hline & 12
\end{tabular}

See footnotes at end of table 
Table 33. Domestic Distribution of U.S. Coal by Origin State, Consumer, Destination and Method of Transportation, January-June 1991 (Continued) (Thousand Short Tons)

\begin{tabular}{|c|c|c|c|c|c|}
\hline \multirow[t]{2}{*}{$\begin{array}{l}\text { State of Destination } \\
\text { by Method of Transportation }\end{array}$} & $\begin{array}{l}\text { Electric } \\
\text { Utillities }\end{array}$ & $\begin{array}{l}\text { Coke } \\
\text { Plants }\end{array}$ & $\begin{array}{l}\text { Industrial } \\
\text { Plants } \\
\text { (Except Coke) }\end{array}$ & $\begin{array}{l}\text { Residential } \\
\text { and Commerclal }\end{array}$ & Total \\
\hline & \multicolumn{5}{|c|}{ ORIGIN: PENNSYLVANIA, BITUMINOUS (Continued) } \\
\hline Minnesota & 329 & - & 18 & - & 348 \\
\hline Railroad & 325 & - & - & - & 325 \\
\hline Great Lakes & - & - & 18 & - & 18 \\
\hline$\ldots \ldots \ldots$ & 5 & - & - & - & 5 \\
\hline New Hampshire $\ldots . .$. & 394 & - & 1 & . & 396 \\
\hline Ralload $\ldots$ & 394 & - & 1 & - & 395 \\
\hline$\ldots \ldots+\cdots \cdots$ & - & - & - & - & - \\
\hline New Jersey & 8 & - & - & - & 8 \\
\hline Raulroad & 8 & - & - & - & 8 \\
\hline Truck... & - & - & - & . & • \\
\hline New York & 2,642 & 409 & 236 & 44 & 3,331 \\
\hline$\ldots \ldots$ & 2.101 & 403 & 111 & 7 & 2,621 \\
\hline . & 228 & - & 13 & - & 241 \\
\hline$\ldots \ldots+\ldots$ & 312 & 6 & 112 & 38 & 468 \\
\hline$\ldots \ldots$ & 1,644 & - & 120 & $\because$ & 1,764 \\
\hline - & 272 & - & - & - & 272 \\
\hline$\ldots \ldots+\ldots$ & 1.126 & - & - & - & 1,126 \\
\hline .. …… & 12 & - & 36 & - & 48 \\
\hline$\ldots \ldots \ldots \ldots$ & 234 & - & 84 & - & 318 \\
\hline Pennsylvania & 14,972 & 1,022 & 929 & 304 & 17,227 \\
\hline Raulroad $\ldots$ & 5.939 & 261 & 31 & 6 & 6.237 \\
\hline River & 532 & 709 & 7 & - & 1,249 \\
\hline Great Lakes & 22 & - & - & - & 22 \\
\hline Tidewater & 34 & - & - & - & 34 \\
\hline Truck ....... & 3.700 & 52 & 866 & 297 & 4,915 \\
\hline Tramway, Conveyor and Slurry Pipeline. & 4.745 & - & - & - & 4.745 \\
\hline Unknown $\ldots \ldots \ldots \ldots \ldots \ldots$ & - & - & 25 & 1 & 26 \\
\hline South Carolina & - & - & 2 & - & 2 \\
\hline$\ldots \ldots \ldots \ldots$ & - & - & 2 & - & 2 \\
\hline Texas $\ldots \ldots \ldots$ & - & - & 2 & - & 2 \\
\hline River & - & - & 2 & - & 2 \\
\hline Truck & - & - & - & • & . \\
\hline$\ldots \ldots$ & - & 153 & - & - & 153 \\
\hline Ralload $\ldots . . . .$. & - & 153 & - & - & 153 \\
\hline Vermont & - & - & - & - & • \\
\hline Truck $\ldots$ & - & - & - & - & - \\
\hline$\ldots \ldots+\ldots \ldots$ & 43 & - & - & . & 43 \\
\hline$\ldots \quad \ldots \quad \ldots \ldots$ & 32 & - & - & - & 32 \\
\hline$\cdots \cdots$ & 11 & - & - & - & 11 \\
\hline West Virginia & 499 & - & 272 & - & 771 \\
\hline Raslroad & 13 & - & - & - & 13 \\
\hline River & 403 & - & 146 & - & 549 \\
\hline$\ldots \ldots \ldots \ldots \ldots$ & 83 & - & 126 & - & 209 \\
\hline Wisconsin & 726 & - & 26 & - & 752 \\
\hline Rallroad & 726 & - & - & - & 726 \\
\hline Great Lakes & - & - & 26 & - & 26 \\
\hline Unknown State & - & - & - & - & 1149 \\
\hline Unknown & - & - & - & - & 1149 \\
\hline State Total & 23,461 & 1,836 & 1,908 & 366 & 27,721 \\
\hline$\ldots \ldots \ldots$ & 11.721 & 1.041 & 345 & 17 & 13,125 \\
\hline River & 2.290 & 709 & 195 & - & 3,194 \\
\hline Great Lakes & 192 & 28 & 101 & - & 322 \\
\hline Tidewater. & 100 & - & - & - & 100 \\
\hline Truck & 4.413 & 58 & 1,241 & 348 & 6.060 \\
\hline Tramway Conveyor and Slurry Pipeline & 4.745 & - & - & - & 4,745 \\
\hline Unknown & - & - & 25 & 1 & 175 \\
\hline
\end{tabular}

ORIGIN: TENNESSEE
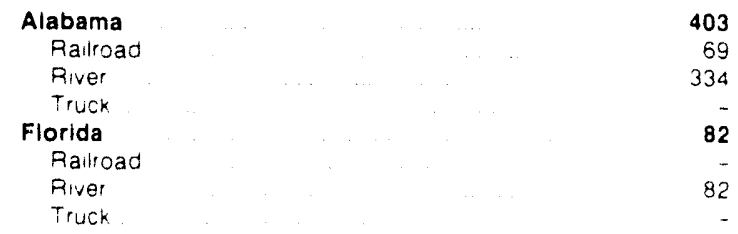

$\begin{array}{rrr}102 & 6 & 512 \\ - & 6 & 76 \\ 102 & - & 436 \\ . & - & . \\ 49 & - & 131 \\ 48 & - & 48 \\ - & - & 82\end{array}$

See footnotes at end of table 
Table 33. Domestic Distribution of U.S. Coal by Origin State, Consumer, Destination and Method of Transportation, January-June 1991 (Continued) (Thousand Short Tons)

\begin{tabular}{|c|c|c|c|c|c|}
\hline $\begin{array}{l}\text { State of Destination } \\
\text { by Method of Transportation }\end{array}$ & $\begin{array}{l}\text { Electric } \\
\text { Utilities }\end{array}$ & $\begin{array}{l}\text { Coke } \\
\text { Plants }\end{array}$ & $\begin{array}{c}\text { Industrial } \\
\text { Plants } \\
\text { (Except Coke) }\end{array}$ & $\begin{array}{l}\text { Residential } \\
\text { and Commercial }\end{array}$ & Total \\
\hline & \multicolumn{5}{|c|}{ ORIGIN: TENNESSEE (Continued) } \\
\hline Georgia & 32 & - & 184 & - & 216 \\
\hline Railroad & 32 & - & 133 & - & 165 \\
\hline 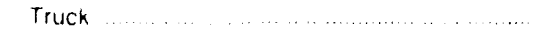 & - & - & 51 & - & 51 \\
\hline Illinois $\ldots \ldots \ldots \ldots$ & 10 & - & 18 & - & 29 \\
\hline Railroad $\ldots \ldots \ldots \ldots$ & 10 & - & - & - & 10 \\
\hline Rivet $\ldots \ldots \ldots$ & - & - & 18 & - & 18 \\
\hline Indiana & - & - & 1 & - & 1 \\
\hline Truck $\quad \ldots \ldots \ldots \ldots \ldots$ & - & - & 1 & - & 1 \\
\hline Kentucky $\ldots \ldots \ldots \ldots \ldots$ & 306 & - & 1 & 1 & 308 \\
\hline Railroad $\ldots \ldots \ldots \ldots$ & 305 & - & - & - & 305 \\
\hline 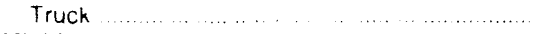 & 1 & - & 1 & 1 & 3 \\
\hline Michigan & - & - & 18 & - & 18 \\
\hline 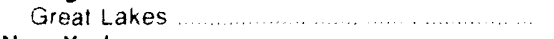 & $\cdot$ & - & 18 & - & 18 \\
\hline New York & - & - & - & 1 & 1 \\
\hline Ralload $\ldots \ldots \ldots \ldots \ldots \ldots \ldots$ & - & - & - & 1 & 1 \\
\hline North Carolina & - & - & 85 & - & 85 \\
\hline$\ldots \ldots \ldots \ldots \ldots \ldots \ldots \ldots \ldots$ & - & - & 69 & - & 69 \\
\hline 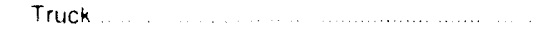 & - & - & 16 & - & 16 \\
\hline 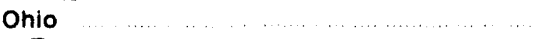 & - & - & - & 1 & 1 \\
\hline$\ldots \ldots \ldots \ldots \ldots \ldots \ldots$ & - & - & - & 1 & 1 \\
\hline South Carolina & - & - & 13 & 15 & 28 \\
\hline Railroad $\ldots \ldots \ldots \ldots$ & - & - & 12 & 15 & 27 \\
\hline$\ldots \ldots, \quad \ldots, \ldots, \quad \ldots \ldots \ldots$ & - & - & 1 & $\cdot$ & 1 \\
\hline 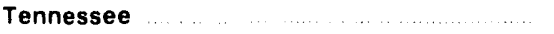 & 802 & - & 150 & 56 & 1,008 \\
\hline$\ldots \ldots \ldots \ldots \ldots \ldots$ & 802 & - & - & 35 & 837 \\
\hline 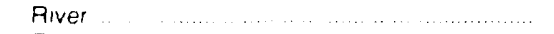 & - & - & - & 3 & 3 \\
\hline Truck $\ldots \ldots \ldots \ldots \ldots$ & - & - & 150 & 18 & 168 \\
\hline Uriknown $\ldots \ldots \ldots \ldots \ldots$ & - & - & - & $"$ & $\cdot$ \\
\hline Virginla $\ldots \ldots, \quad \ldots \ldots$ & - & - & 8 & - & 8 \\
\hline$\ldots \quad \ldots \ldots \ldots \ldots \ldots \ldots \ldots \ldots \ldots \ldots$ & - & - & 8 & - & 8 \\
\hline Unknown State $\ldots \ldots$ & - & - & - & - & 144 \\
\hline Unknown $\quad \ldots \ldots \ldots \ldots \ldots \ldots$ & - & - & - & - & 144 \\
\hline State Total & 1,636 & - & 629 & 79 & 12,388 \\
\hline Rautroad $\ldots \ldots \ldots$ & 1.218 & - & 263 & 57 & 1.538 \\
\hline 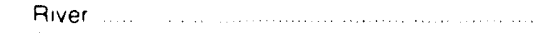 & 416 & - & 121 & 3 & 539 \\
\hline Great Lakes $\quad \ldots \ldots \ldots \ldots$ & $\cdot$ & - & 18 & - & 18 \\
\hline Truck $\ldots \ldots \ldots \ldots$ & 1 & - & 227 & 19 & 248 \\
\hline Unknown $\ldots \ldots \ldots \ldots \ldots \ldots \ldots \ldots$ & - & - & - & • & 44 \\
\hline
\end{tabular}

\begin{tabular}{|c|c|}
\hline Texas & 24,941 \\
\hline Railroad & 10.570 \\
\hline Truck & 6.403 \\
\hline Tramway, Conveyor and Slurry $D_{1}$ pelıne & 7,968 \\
\hline State Total & 24,941 \\
\hline Railroad & 10,570 \\
\hline Truck & 6.403 \\
\hline Tramway. Coriveyor. and Slurry Pipeline & 7.968 \\
\hline
\end{tabular}

ORIGIN: TEXAS

$\begin{array}{llr}917 & - & \mathbf{2 5 , 8 5 8} \\ 144 & - & 10,714 \\ 118 & - & 6,521 \\ 655 & - & 8,623 \\ 917 & & \\ 144 & - & \mathbf{2 5 , 8 5 8} \\ 118 & - & 10,714 \\ 655 & - & 6,521 \\ & - & 8,623\end{array}$

ORIGIN: UTAH

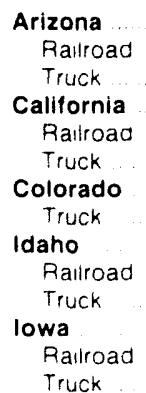

See footnotes at end of table

\begin{tabular}{|c|c|c|}
\hline 56 & - & 57 \\
\hline 56 & - & 56 \\
\hline- & -. & 1 \\
\hline 1,209 & 43 & 1,252 \\
\hline 1,209 & 43 & 1,252 \\
\hline • & - & 1 \\
\hline- & 1 & 1 \\
\hline- & 1 & 1 \\
\hline 20 & 32 & 52 \\
\hline- & 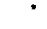 & 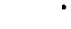 \\
\hline 20 & 32 & 51 \\
\hline- & * & • \\
\hline- & - & 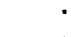 \\
\hline
\end{tabular}


Table 33. Domestic Distribution of U.S. Coal by Origin State, Consumer, Destination and Method of Transportation, January-June 1991 (Continued)

(Thousand Short Tons)

\begin{tabular}{|c|c|c|c|c|c|}
\hline \multirow[t]{2}{*}{$\begin{array}{l}\text { State of Destination } \\
\text { by Method of Transportation }\end{array}$} & $\begin{array}{l}\text { Electric } \\
\text { Utilities }\end{array}$ & $\begin{array}{l}\text { Coke } \\
\text { Plants }\end{array}$ & $\begin{array}{c}\text { Industrial } \\
\text { Plants } \\
\text { (Except Coke) }\end{array}$ & $\begin{array}{c}\text { Residential } \\
\text { and Commercial }\end{array}$ & Total \\
\hline & \multicolumn{5}{|c|}{ ORIGIN: UTAH (Continued) } \\
\hline Montana & - & - & 19 & - & 19 \\
\hline Truck & - & - & 19 & & 19 \\
\hline Nevada & 1.422 & - & 113 & 1 & 1,536 \\
\hline Railioad & 1.422 & - & 92 & - & 1515 \\
\hline Truck . & - & - & 21 & 1 & 22 \\
\hline Oregon & - & - & - & 1 & 1 \\
\hline Truck & - & - & - & $i$ & : \\
\hline Utah & 5,935 & 301 & 278 & 161 & 6,675 \\
\hline Ralload & 2,279 & 301 & & - & 2580 \\
\hline Truck & 2.462 & - & 276 & 160 & 2.898 \\
\hline Tramway. Conveyor. and Slury Pipeline & 1.194 & - & - & - & 1194 \\
\hline Unknown & - & - & 1 & 2 & 3 \\
\hline Washington & - & - & 58 & 30 & 88 \\
\hline Raltroad & - & - & 58 & 29 & 97 \\
\hline Truck & - & - & · & • & $\cdot$ \\
\hline Wyoming & - & - & 35 & • & 35 \\
\hline Truck & - & - & 35 & $\cdot$ & 35 \\
\hline State Total & 7,358 & 301 & 1,787 & 269 & 9,715 \\
\hline Rallroad & 3.701 & 301 & 1.415 & 73 & 5.490 \\
\hline Truck & 2.463 & - & 371 & 194 & 3.028 \\
\hline Tramway. Conveyor and Slurry Pipeline & 1,194 & - & - & - & 1.194 \\
\hline \multirow{2}{*}{ Unknown } & - & - & 1 & 2 & 3 \\
\hline & \multicolumn{5}{|c|}{ ORIGIN: VIRGINIA } \\
\hline Alabama & 26 & 258 & 96 & - & 379 \\
\hline Raltroad & 26 & 258 & 96 & - & 379 \\
\hline Colorado & • & - & - & - & • \\
\hline Pailroad & . & - & - & - & . \\
\hline Delaware & 84 & - & - & - & 84 \\
\hline Ralload & 84 & - & - & - & 84 \\
\hline Florida & 158 & - & 76 & - & 234 \\
\hline Railroad & 158 & - & 76 & - & 234. \\
\hline Truck & - & - & • & - & \\
\hline Georgia & 1,443 & - & 187 & 1 & 1,631 \\
\hline Railroad & 1443 & - & 187 & 1 & 1.631 \\
\hline Illinois & - & 312 & - & - & 312 \\
\hline Raltroad & - & 138 & - & - & 138 \\
\hline River & - & 174 & - & - & 174 \\
\hline Indiana & - & 602 & - & - & 602 \\
\hline Raltroad & - & 556 & - & - & 556 \\
\hline River & - & 46 & - & - & 46 \\
\hline Maryland & - & 79 & - & - & 79 \\
\hline Ratiroad & - & 42 & - & - & 42 \\
\hline Tidewater & • & 36 & - & - & 36 \\
\hline Truck & - & - & - & . & ? \\
\hline Massachusetts & 316 & - & - & - & 316 \\
\hline Tidewate' & 316 & - & - & - & 396 \\
\hline Michigan & 2 & - & - & - & 2 \\
\hline Ralroad & 2 & - & - & - & 2 \\
\hline New Hampshire & 186 & - & - & - & 186 \\
\hline Tidewater & 186 & - & - & - & 136 \\
\hline New Jersey & 210 & - & - & - & 210 \\
\hline Falload & 15 & - & - & - & 15 \\
\hline Tidewater & .95 & - & - & - & 95 \\
\hline New York & - & - & 73 & - & 73 \\
\hline Panroas & - & - & 73 & - & 73 \\
\hline North Carolina & 1,893 & - & 168 & 15 & 2.076 \\
\hline Raviroad & +893 & - & 147 & $\Xi$ & 2053 \\
\hline Tes: & - & - & 21 & .. & 2 \\
\hline Onio & - & 397 & 14 & - & 411 \\
\hline Gairoas & - & 290 & 14 & .. & 304 \\
\hline Aive. & - & 107 & $\therefore$ & - & 0 \\
\hline Pennsylvania & 18 & 452 & 3 & $\cdot$ & 473 \\
\hline Rarroad & - & 172 & 3 & & 175 \\
\hline Giver & 15 & 280 &. & & 298 \\
\hline TuE & - & - & - & $\cdot$ & \\
\hline
\end{tabular}


Table 33. Domestic Distribution of U.S. Coal by Origin State, Consumer, Destination and Method of Transportation, January-June 1991 (Continued) (Thousand Short Tons)

\begin{tabular}{|c|c|c|c|c|c|}
\hline $\begin{array}{l}\text { State of Destination } \\
\text { by Method of Transportation }\end{array}$ & $\begin{array}{l}\text { Electric } \\
\text { Utilitios }\end{array}$ & $\begin{array}{l}\text { Coke } \\
\text { Plants }\end{array}$ & $\begin{array}{c}\text { Industrial } \\
\text { Plants } \\
\text { (Except Coke) }\end{array}$ & $\begin{array}{l}\text { Residential } \\
\text { and Commercial }\end{array}$ & Total \\
\hline & \multicolumn{5}{|c|}{ ORIGIN: VIRGINIA (Continued) } \\
\hline South Carolina & 457 & - & 332 & - & 789 \\
\hline Railroad ............ & 457 & - & 330 & - & 787 \\
\hline 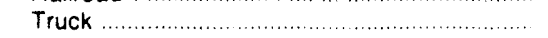 & - & - & 2 & - & 2 \\
\hline (1) & 636 & - & 649 & 16 & 1,301 \\
\hline (1) & 636 & - & 648 & 15 & 1,299 \\
\hline (2) & - & - & - & 1 & 1 \\
\hline Texas & - & .. & - & 6 & 6 \\
\hline Railroad & - & - & - & 6 & 6 \\
\hline Utah & - & 58 & - & - & 58 \\
\hline Raitroad & - & 58 & - & - & 58 \\
\hline Vermont & - & - & 7 & - & 7 \\
\hline Railroad & - & - & 7 & - & 7 \\
\hline Virginia & 1,600 & 480 & 452 & 7 & 22,539 \\
\hline Railroad & 1,600 & - & 427 & 3 & 2,030 \\
\hline Truck & - & - & 13 & 2 & 15 \\
\hline Tramway, Conveyor, and Slurry Pipeline & - & 480 & - & - & 480 \\
\hline 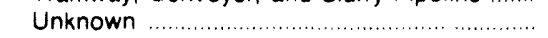 & - & - & 13 & 3 & 216 \\
\hline West Virginia & 7 & 408 & 2 & 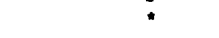 & 417 \\
\hline Railroad & 7 & - & 1 & • & 8 \\
\hline 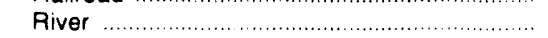 & - & 408 & - & - & 408 \\
\hline 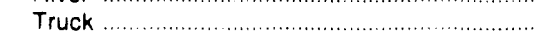 & - & - & 1 & - & 1 \\
\hline 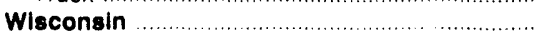 & 43 & - & - & • & 43 \\
\hline (n) & 21 & - & - & • & 21 \\
\hline River .................... & 22 & - & - & - & 22 \\
\hline Unknown State. & - & - & - & - & 124 \\
\hline 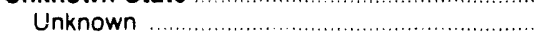 & - & - & - & - & 124 \\
\hline State Total & 7,079 & 3,044 & 2,058 & 46 & 3 12,252 \\
\hline Railroad & 6,342 & 1,514 & 2.009 & 39 & 9,905 \\
\hline River ......... & 40 & 1,014 & - & - & 1,054 \\
\hline Tidewater & 696 & 36 & - & - & 733 \\
\hline Truck & - & - & 37 & 3 & 40 \\
\hline Tramway, Conveyor, and Slurry Pipeline ........ & - & 480 & - & - & 480 \\
\hline \multirow[t]{2}{*}{ 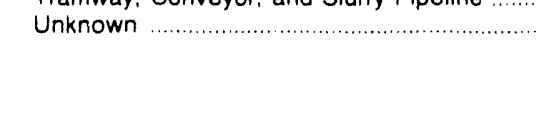 } & - & - & 13 & 3 & 30 \\
\hline & \multicolumn{5}{|c|}{ ORIGIN: WASHINGTON } \\
\hline Washington .. & 2,193 & - & - & * & 2,193 \\
\hline 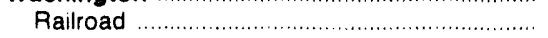 & 2,193 & - & - & - & 2,193 \\
\hline 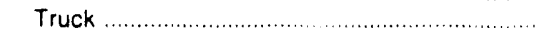 & 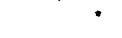 & - & - & * & * \\
\hline State Totul & 2,193 & - & - & * & 2,193 \\
\hline Railroad & 2,193 & - & - & - & 2,193 \\
\hline \multirow[t]{3}{*}{ 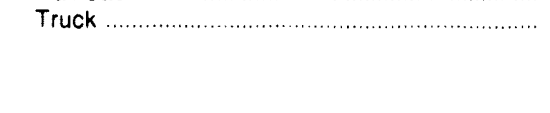 } & 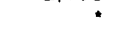 & - & - & • & - \\
\hline & & & & & \\
\hline & \multicolumn{5}{|c|}{ ORIGIN: WEST VIRGINIA, TOTAL } \\
\hline Alabama $\ldots \ldots \ldots \ldots$ & 440 & 241 & 185 & - & 866 \\
\hline Railroad & 166 & 240 & 149 & - & 555 \\
\hline River & 83 & - & 36 & - & 120 \\
\hline 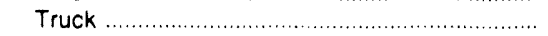 & 191 & $\cdot$ & " & - & 191 \\
\hline California & - & - & • & - & $*$ \\
\hline 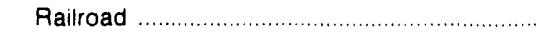 & - & - & $\cdot$ & - & - \\
\hline Delaware & 604 & - & 2 & - & 605 \\
\hline 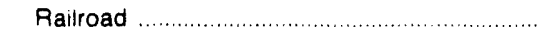 & 604 & - & - & - & 604 \\
\hline Truck & - & - & 2 & - & 2 \\
\hline District of Columbla & - & - & - & 9 & 9 \\
\hline 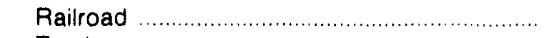 & - & - & - & 3 & 3 \\
\hline 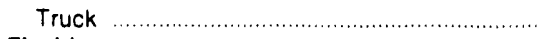 & - & - & - & 6 & 6 \\
\hline Florida & 978 & - & - & - & 978 \\
\hline 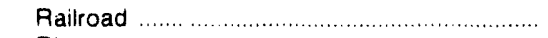 & 402 & - & - & - & 402 \\
\hline 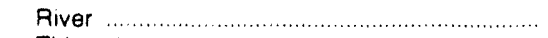 & 2 & - & + & - & 2 \\
\hline 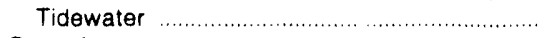 & 574 & - & - &.- & 574 \\
\hline 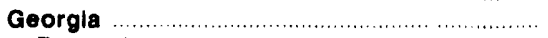 & 1,062 & - & - & - & 1,062 \\
\hline Railroad & 1.062 & - & - & - & 1.062 \\
\hline inols & 383 & 319 & 55 & * & 757 \\
\hline 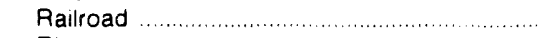 & 150 & 178 & 18 & - & 346 \\
\hline River & 233 & 141 & 33 & - & $40 ?$ \\
\hline Truck & - & - & 5 & • & 5 \\
\hline
\end{tabular}

See footnotes at end of table. 
Table 33. Domestic Distribution of U.S. Coal by Origin State, Consumer, Destination and Method of Transportation, January-June 1991 (Continued) (Thousand Short Tons)

\begin{tabular}{|c|c|c|c|c|c|}
\hline $\begin{array}{l}\text { State of Destination } \\
\text { by Method of Transportation }\end{array}$ & $\begin{array}{l}\text { Electric } \\
\text { L'tilitles }\end{array}$ & $\begin{array}{l}\text { Coke } \\
\text { Plante }\end{array}$ & $\begin{array}{c}\text { Industrlal } \\
\text { Plants } \\
\text { (Except Coke) }\end{array}$ & $\begin{array}{l}\text { Residential } \\
\text { and Commercial }\end{array}$ & Total \\
\hline & \multicolumn{5}{|c|}{ ORIGIN: WEST VIRGINIA, TOTAL (Continued) } \\
\hline Indiana & 131 & 1,156 & 549 & - & 1,837 \\
\hline 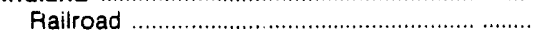 & 129 & 1.156 & 381 & - & 1,665 \\
\hline 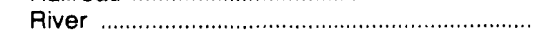 & - & - & 139 & - & 139 \\
\hline Truck & 2 & - & 29 & - & 32 \\
\hline 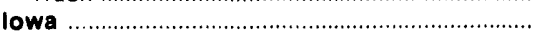 & - & - & 20 & - & 20 \\
\hline 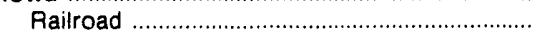 & - & - & 8 & - & 8 \\
\hline 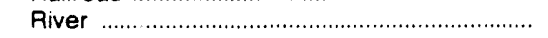 & - & - & 12 & - & 12 \\
\hline 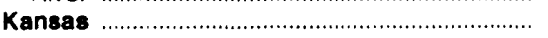 & - & - & 5 & - & 5 \\
\hline 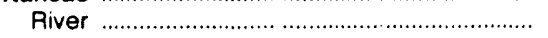 & - & - & 5 & - & 5 \\
\hline 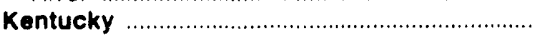 & 1,532 & 355 & 306 & - & 2,192 \\
\hline 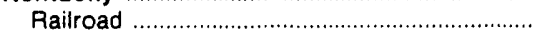 & 595 & 307 & 109 & - & 1,010 \\
\hline 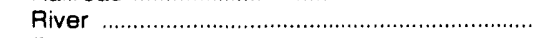 & 784 & 48 & 40 & - & 872 \\
\hline 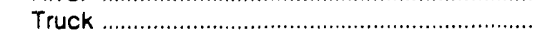 & 153 & - & 157 & - & 309 \\
\hline 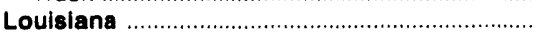 & 2 & - & 12 & - & 14 \\
\hline 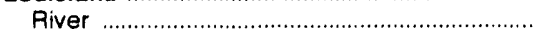 & 2 & - & 12 & - & 14 \\
\hline 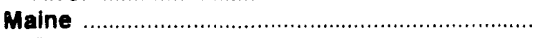 & - & - & 10 & - & 10 \\
\hline 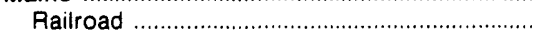 & - & - & 10 & - & 10 \\
\hline Maryland & 2,763 & 54 & 265 & 10 & 3,091 \\
\hline 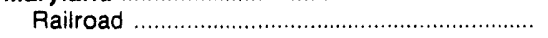 & 2,371 & 54 & 226 & - & 2,650 \\
\hline 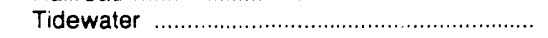 & 388 & - & - & - & 388 \\
\hline Truck & 5 & - & 40 & 10 & 54 \\
\hline 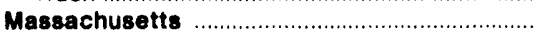 & 920 & - & 17 & - & 938 \\
\hline 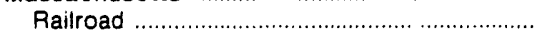 & 337 & - & 17 & - & 354 \\
\hline 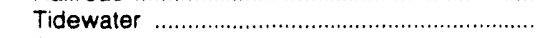 & 563 & - & - & - & 563 \\
\hline 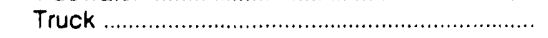 & 20 & - & 1 & - & 21 \\
\hline 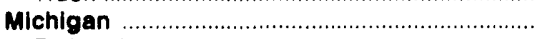 & 2,997 & 181 & 457 & 3 & 3,637 \\
\hline 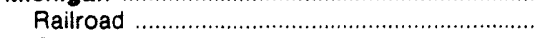 & 2,987 & 157 & 389 & 2 & 3,535 \\
\hline 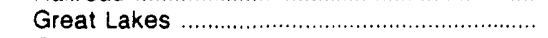 & 9 & 24 & 65 & 1 & 100 \\
\hline 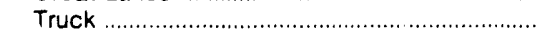 & - & - & 2 & * & 3 \\
\hline 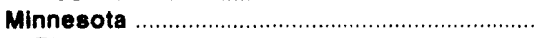 & - & - & 18 & - & 18 \\
\hline 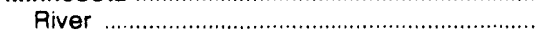 & - & - & 18 & - & 18 \\
\hline 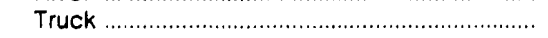 & - & - & $\cdot$ & - & $\bullet$ \\
\hline 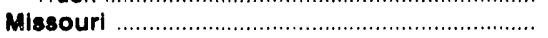 & - & - & 51 & 4 & 55 \\
\hline 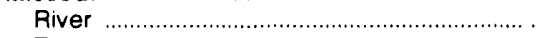 & - & $\ddot{-}$ & 47 & 2 & 48 \\
\hline 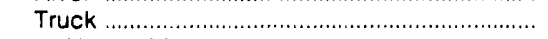 & - & - & 4 & 3 & 7 \\
\hline 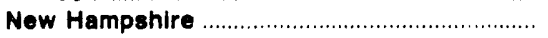 & 123 & - & - & - & 123 \\
\hline 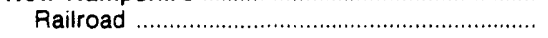 & 80 & - & - & - & 80 \\
\hline 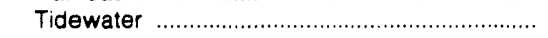 & 43 & - & - & - & 43 \\
\hline 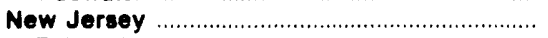 & 596 & - & 124 & - & 720 \\
\hline 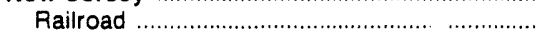 & 359 & - & 124 & - & 484 \\
\hline 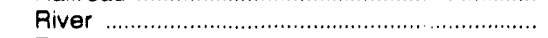 & 41 & - & - & - & 41 \\
\hline 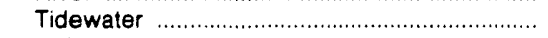 & 196 & - & - & - & 196 \\
\hline 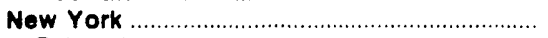 & 1,638 & 58 & 489 & 9 & 2,195 \\
\hline 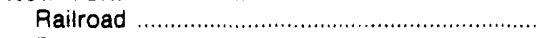 & 1,633 & 58 & 434 & 4 & 2,129 \\
\hline 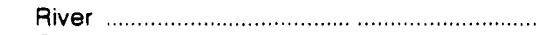 & - & - & 55 & - & 55 \\
\hline 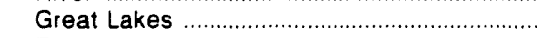 & 6 & - & - & - & 6 \\
\hline 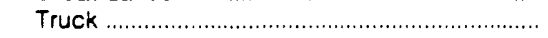 & - & - & $\cdot$ & 5 & 5 \\
\hline 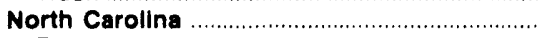 & 2,564 & - & 206 & 18 & 2,788 \\
\hline 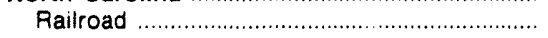 & 2,564 & - & 200 & 18 & 2,782 \\
\hline 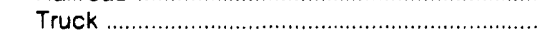 & - & - & 6 & - & 6 \\
\hline North Dakota & - & - & 1 & - & 1 \\
\hline 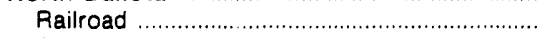 & - & - & 1 & - & 1 \\
\hline Ohio & 6,986 & 1,084 & 387 & 28 & 8,485 \\
\hline 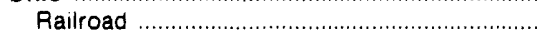 & 1,213 & 1,084 & 186 & 2 & 2,484 \\
\hline 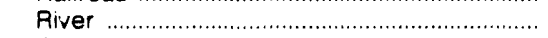 & 5,590 & - & 47 & - & 5,637 \\
\hline 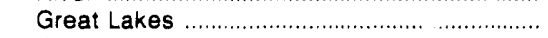 & 132 & - & 30 & - & 162 \\
\hline 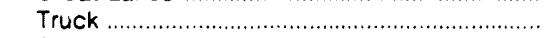 & 51 & - & 124 & 26 & 201 \\
\hline 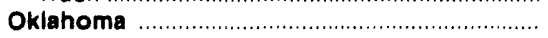 & - & - & 9 & - & 9 \\
\hline 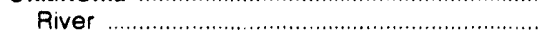 & - & - & 9 & - & 9 \\
\hline 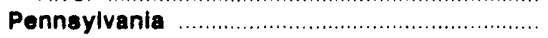 & 4,886 & 1,069 & 388 & 6 & 6,350 \\
\hline 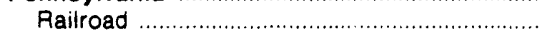 & 307 & 534 & 178 & 1 & 1,019 \\
\hline 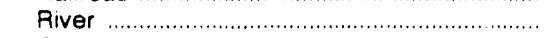 & 4.543 & 536 & 37 & - & 5.115 \\
\hline Truck & 37 & - & 173 & 5 & 216 \\
\hline South Carolina & 55 & - & 34 & - & 90 \\
\hline 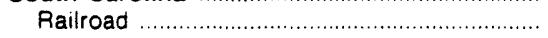 & 55 & - & 33 & - & 89 \\
\hline 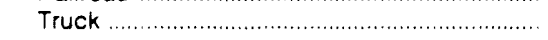 & - & - & 1 & - & 1 \\
\hline
\end{tabular}

See footnotes at end of table. 
Table 33. Domestic Distribution of U.S. Coal by Origin State, Consumer, Destination and Method of Transportation, January-June 1991 (Continued) (Thousand Short Tons)

\begin{tabular}{|c|c|c|c|c|c|}
\hline $\begin{array}{l}\text { State of Destination } \\
\text { by Method of Transportation }\end{array}$ & $\begin{array}{l}\text { Electric } \\
\text { Utilities }\end{array}$ & $\begin{array}{l}\text { Coke } \\
\text { Plants }\end{array}$ & $\begin{array}{c}\text { Industrlal } \\
\text { Plants } \\
\text { (Except Coke) }\end{array}$ & $\begin{array}{c}\text { Residential } \\
\text { and Commercial }\end{array}$ & Total \\
\hline & \multicolumn{5}{|c|}{ ORIGIN: WEST VIRGINIA, TOTAL (Continued) } \\
\hline South Dakota. & - & - & 5 & - & 5 \\
\hline 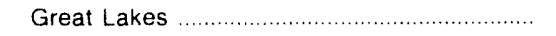 & - & - & 5 & - & 5 \\
\hline Tennessee & 551 & - & 33 & 8 & 592 \\
\hline 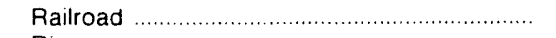 & - & - & 16 & $\overline{-}$ & 16 \\
\hline 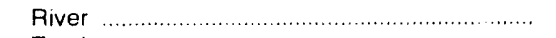 & 551 & - & 17 & 2 & 570 \\
\hline 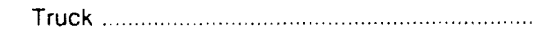 & - & - & - & 6 & 6 \\
\hline Utah & - & 35 & - & - & 35 \\
\hline 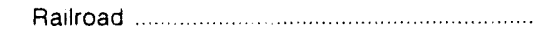 & - & 35 & - & - & 35 \\
\hline Virginia & 1,768 & - & 504 & 34 & 2,306 \\
\hline 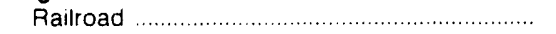 & 1,641 & - & 383 & 25 & 2,049 \\
\hline 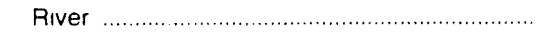 & - & - & - & 1 & 1 \\
\hline 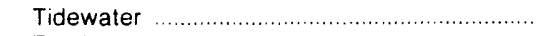 & 5 & - & 7 & - & 12 \\
\hline 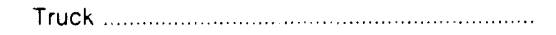 & 122 & - & 114 & 8 & 244 \\
\hline West Virginia & 12,116 & 1,507 & 717 & 125 & 214,469 \\
\hline 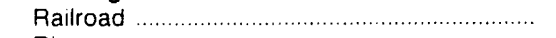 & 5,914 & 877 & 115 & - & 6,907 \\
\hline 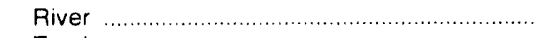 & 2,131 & 630 & 191 & - & 2,952 \\
\hline 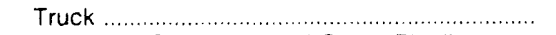 & 1,788 & - & 360 & 118 & 2,266 \\
\hline Tramway, Conveyor, and Slurry Pipeline ....... & 2,282 & - & - & - & 2,282 \\
\hline 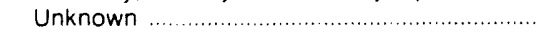 & - & - & 51 & 7 & 262 \\
\hline Wisconsin & 286 & - & 79 & - & 365 \\
\hline 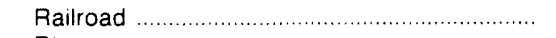 & 270 & - & - & - & 270 \\
\hline 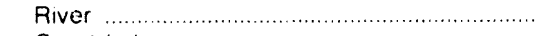 & - & - & 26 & - & 26 \\
\hline 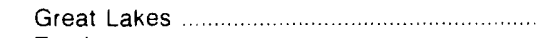 & 17 & - & 44 & - & 61 \\
\hline 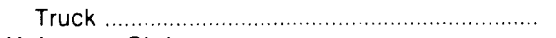 & - & - & 9 & - & 9 \\
\hline 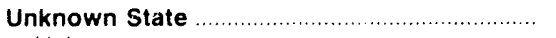 & - & - & - & - & 1368 \\
\hline 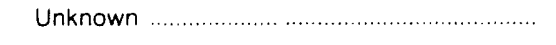 & - & - & - & - & 1368 \\
\hline State Total & 43,380 & 6,058 & 4,929 & 254 & ${ }^{3} 54,994$ \\
\hline 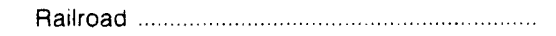 & 22,836 & 4,680 & 2.976 & 55 & 30,547 \\
\hline 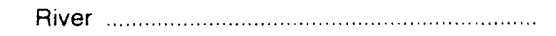 & 13,960 & 1,354 & 724 & 4 & 16,043 \\
\hline 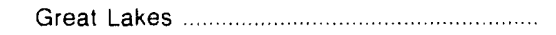 & 164 & 24 & 144 & 1 & 334 \\
\hline 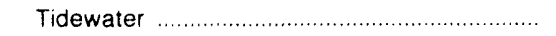 & 1,769 & - & 7 & - & 1.775 \\
\hline 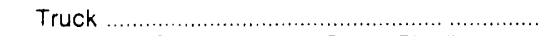 & 2,369 & * & 1.027 & 188 & 3,583 \\
\hline Tramway, Conveyor, and Slurry Pipeline ....... & 2,282 & - & - & - & 2,282 \\
\hline \multirow[t]{3}{*}{ 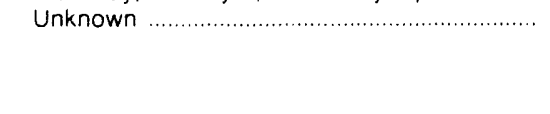 } & - & - & 51 & 7 & 3430 \\
\hline & & & & & \\
\hline & \multicolumn{5}{|c|}{ ORIGIN: WEST VIRGINIA, NORTHERN } \\
\hline California & - & - & $*$ & - & * \\
\hline 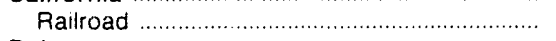 & - & - & $\cdot$ & - & * \\
\hline 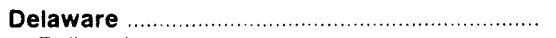 & 238 & - & 2 & - & 240 \\
\hline 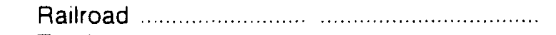 & 238 & - & - & - & 238 \\
\hline 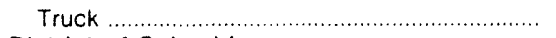 & - & - & 2 & - & 2 \\
\hline District of Columbia & - & - & - & 6 & 6 \\
\hline 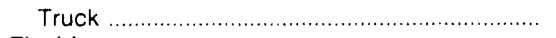 & - & - & - & 6 & 6 \\
\hline 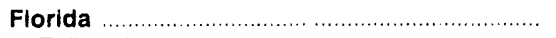 & 217 & - & - & - & 217 \\
\hline 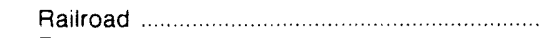 & 7 & - & - & - & 7 \\
\hline 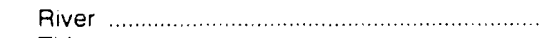 & $\cdot$ & - & - & - & * \\
\hline 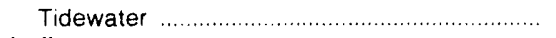 & 209 & - & - & - & 209 \\
\hline Indiana & - & - & 161 & - & 161 \\
\hline 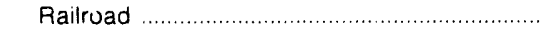 & - & - & 161 & - & 161 \\
\hline lowa & - & - & 1 & - & 1 \\
\hline 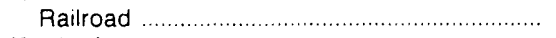 & - & - & 1 & - & 1 \\
\hline 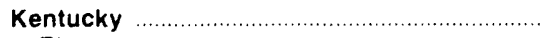 & 9 & - & - & - & 9 \\
\hline 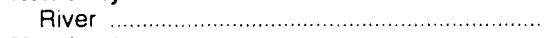 & 9 & - & - & - & 9 \\
\hline 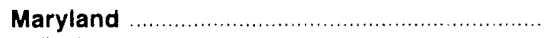 & 2,030 & - & 119 & 10 & 2,159 \\
\hline 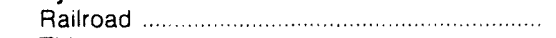 & 1,824 & - & 80 & - & 1.904 \\
\hline 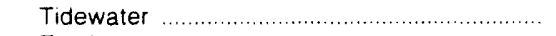 & 206 & - & - & - & 206 \\
\hline 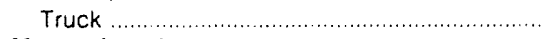 & - & - & 40 & 10 & 49 \\
\hline 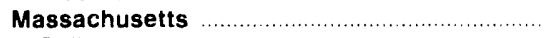 & 835 & - & 1 & - & 836 \\
\hline 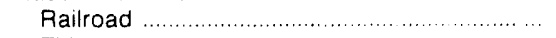 & 304 & - & 1 & - & 305 \\
\hline 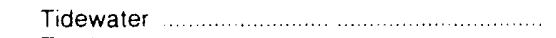 & 528 & - & - & - & 528 \\
\hline 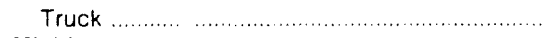 & 3 & - & - & - & 3 \\
\hline 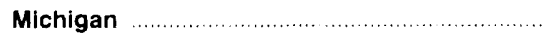 & 4 & - & 17 & - & 21 \\
\hline 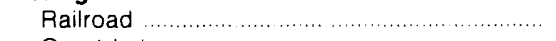 & 4 & - & - & - & 4 \\
\hline 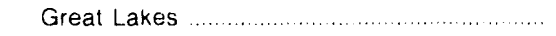 & - & - & 17 & - & 17 \\
\hline
\end{tabular}

See footnotes at end of table. 
Table 33. Domestic Distribution of U.S. Coal by Origin State, Consumer, Destination and Method of Transportation, January-June 1991 (Continued) (Thousand Short Tons)

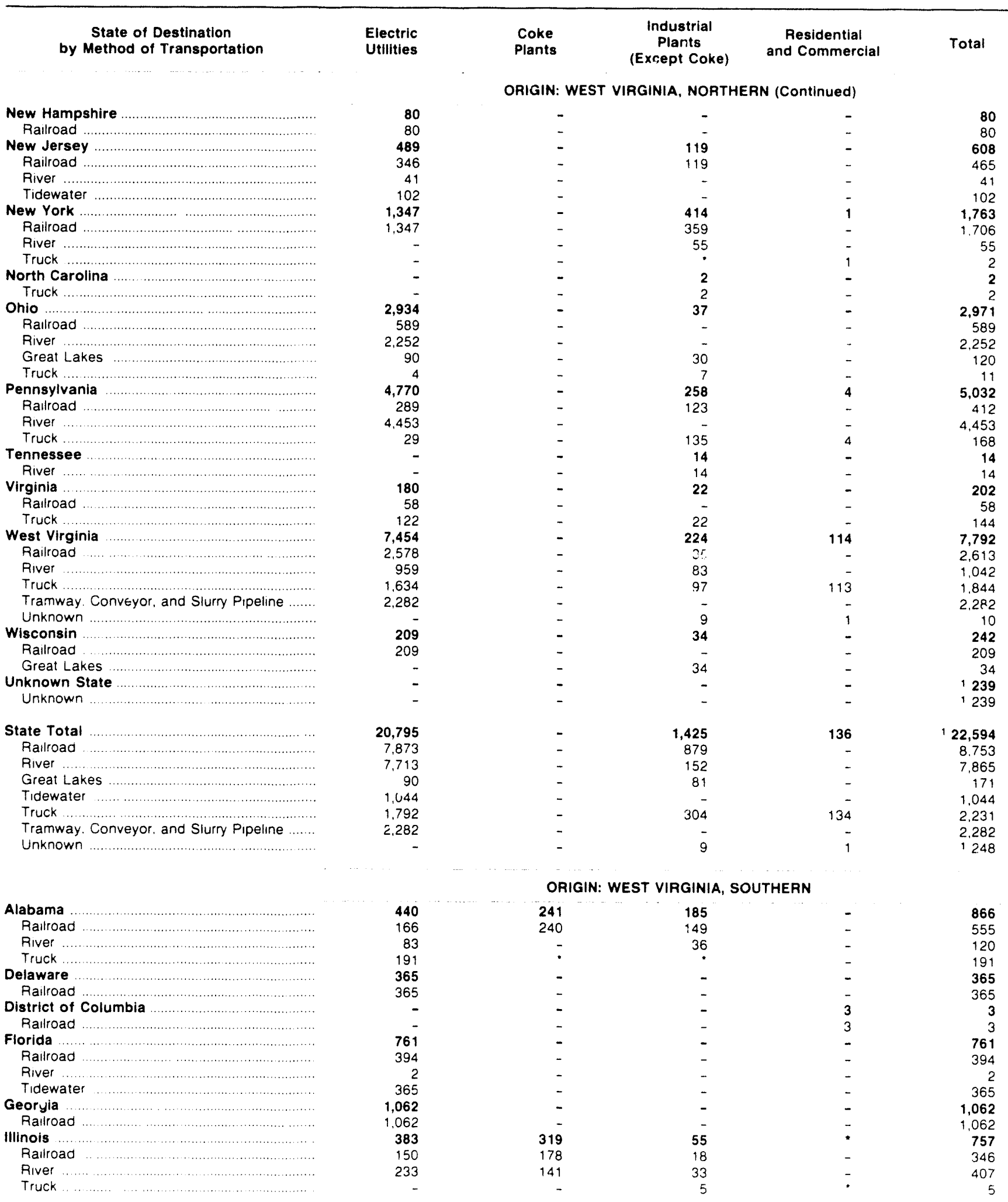

See footnotes at end of table. 
Table 33. Domestic Distribution of U.S. Coal by Origin State, Consumer, Destination and Method of Transportation, January-June 1991 (Continued)

(Thousand Short Tons)

\begin{tabular}{|c|c|c|c|c|c|}
\hline $\begin{array}{l}\text { State of Destination } \\
\text { by Method of Transportation }\end{array}$ & $\begin{array}{l}\text { Electric } \\
\text { Utilities }\end{array}$ & $\begin{array}{l}\text { Coke } \\
\text { Plants }\end{array}$ & $\begin{array}{c}\text { Industrial } \\
\text { Plants } \\
\text { (Except Coke) }\end{array}$ & $\begin{array}{l}\text { Residential } \\
\text { and Commercial }\end{array}$ & Total \\
\hline & \multicolumn{5}{|c|}{ ORIGIN: WEST VIRGINIA, SOUTHERN (Continued) } \\
\hline Indiana & 131 & 1,156 & 388 & - & 1,675 \\
\hline $\begin{array}{l}\text { Railroad } \\
\text { River }\end{array}$ & 129 & 1,156 & 219 & - & 1,504 \\
\hline $\begin{array}{l}\text { River } \\
\text { Truck }\end{array}$ & - & - & 139 & - & 139 \\
\hline $\begin{array}{l}\text { Truck } \\
\text { lowa }\end{array}$ & 2 & - & 29 & - & 32 \\
\hline $\begin{array}{l}\text { lowa } \\
\text { Railroad }\end{array}$ & - & - & 19 & - & 19 \\
\hline $\begin{array}{l}\text { Railroad } \\
\text { River }\end{array}$ & - & - & 7 & - & 7 \\
\hline $\begin{array}{r}\text { River } \\
\text { Kansas }\end{array}$ & - & - & 12 & - & 12 \\
\hline $\begin{array}{r}\text { Kansas } \\
\text { River }\end{array}$ & - & - & 5 & - & 5 \\
\hline $\begin{array}{l}\text { River } \\
\text { Kentucky }\end{array}$ & - & - & 5 & - & 5 \\
\hline $\begin{array}{l}\text { Kentucky } \\
\text { Railroad }\end{array}$ & 1,523 & 355 & 306 & - & 2,183 \\
\hline $\begin{array}{l}\text { Rairoad } \\
\text { River }\end{array}$ & 595 & 307 & 109 & - & 1,010 \\
\hline $\begin{array}{l}\text { River } \\
\text { Truck }\end{array}$ & 775 & 48 & 40 & - & 863 \\
\hline $\begin{array}{l}\text { Truck } \\
\text { Loulsiana }\end{array}$ & 153 & - & 157 & - & 309 \\
\hline (1) & 2 & - & 12 & - & 14 \\
\hline (1) & 2 & - & 12 & - & 14 \\
\hline $\begin{array}{l}\text { Maine } \\
\text { Railroad }\end{array}$ & - & - & 10 & - & 10 \\
\hline Railroad & - & - & 10 & - & 10 \\
\hline Maryland . . . . . . & 733 & 54 & 146 & - & 933 \\
\hline Raulroad & 546 & 54 & 146 & - & 746 \\
\hline $\begin{array}{l}\text { Tidewater } \\
\text { Truck }\end{array}$ & 182 & - & - & - & 182 \\
\hline Truck & 5 & - & - & - & 5 \\
\hline Massachusetts $\ldots$ & 85 & - & 16 & - & 101 \\
\hline Railroad & 33 & - & 16 & - & 48 \\
\hline Tidewater & 36 & - & - & - & 36 \\
\hline Truck & 17 & - & 1 & - & 17 \\
\hline Michigan & 2,993 & 181 & 439 & 3 & 3,616 \\
\hline Railroad & 2,983 & 157 & 389 & 2 & 3,531 \\
\hline Great Lakes & 9 & 24 & 48 & 1 & 82 \\
\hline Truck & - & - & 2 & • & 3 \\
\hline Minnesota & - & - & 18 & - & 18 \\
\hline River & - & - & 18 & - & 18 \\
\hline 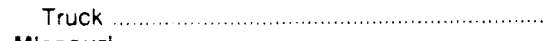 & - & - & • & - & \\
\hline Missouri … & - & - & 51 & 4 & 55 \\
\hline 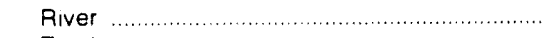 & - & - & 47 & 2 & 48 \\
\hline Truck & - & - & 4 & 3 & 7 \\
\hline New Hampshire & 43 & - & - & - & 43 \\
\hline Tidewater & 43 & - & - & - & 43 \\
\hline 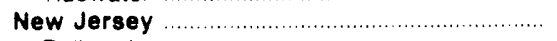 & 107 & - & 6 & - & 112 \\
\hline Railroad & 13 & - & 6 & - & 19 \\
\hline 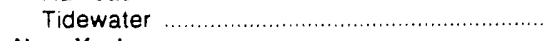 & 94 & - & - & - & 94 \\
\hline New York & 292 & 58 & 75 & 8 & 432 \\
\hline Railroad & 286 & 58 & 75 & 4 & 423 \\
\hline 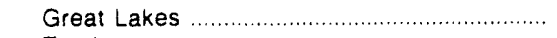 & 6 & - & - & - & 6 \\
\hline 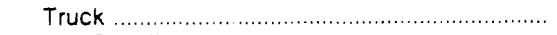 & - & - & - & 4 & 4 \\
\hline North Carolina & 2,564 & - & 204 & 18 & 2,786 \\
\hline 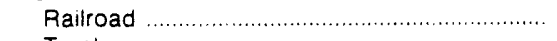 & 2,564 & - & 200 & 18 & 2,782 \\
\hline Truck & - & - & 4 & - & 4 \\
\hline North Dakota & - & - & 1 & - & 1 \\
\hline 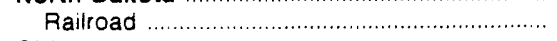 & - & - & 1 & - & 1 \\
\hline Ohio & 4,053 & 1,084 & 349 & 28 & 5,514 \\
\hline 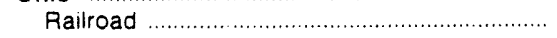 & 624 & 1,084 & 186 & 2 & 1,895 \\
\hline River & 3,338 & - & 47 & - & 3,386 \\
\hline Great Lakes & 43 & - & - & - & 43 \\
\hline 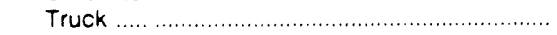 & 47 & - & 116 & 26 & 190 \\
\hline Oklahoma & - & - & 9 & - & 9 \\
\hline River & - & - & 9 & - & 9 \\
\hline Pennsylvania & 116 & 1,069 & 130 & 2 & 1,318 \\
\hline Railroad & 18 & 534 & 55 & 1 & 607 \\
\hline River & 90 & 536 & 37 & - & 663 \\
\hline Truck & 8 & - & 39 & 1 & 48 \\
\hline South Carolina & 55 & - & 34 & - & 90 \\
\hline Railroad & 55 & - & 33 & - & 89 \\
\hline Truck & - & - & 1 & - & 1 \\
\hline South Dakuta & - & - & 5 & - & 5 \\
\hline 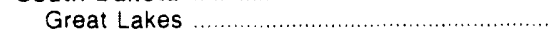 & - & - & 5 & - & 5 \\
\hline Tennessee & 551 & - & 19 & 8 & 579 \\
\hline 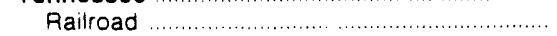 & - & - & 16 & - & 16 \\
\hline River & 551 & - & 3 & 2 & 556 \\
\hline 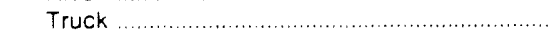 & - & - & - & 6 & 6 \\
\hline
\end{tabular}

See footnotes at end of table 
Table 33. Domestic Distribution of U.S. Coal by Origin State, Consumer, Destination and Method of Transportation, January-June 1991 (Continued) (Thousand Short Tons)

\begin{tabular}{|c|c|c|c|c|c|}
\hline $\begin{array}{l}\text { State of Destination } \\
\text { by Method of Transportation }\end{array}$ & $\begin{array}{l}\text { Electric } \\
\text { Utilities }\end{array}$ & $\begin{array}{l}\text { Coke } \\
\text { Plants }\end{array}$ & $\begin{array}{c}\text { Industrial } \\
\text { Plants } \\
\text { (Except Coke) }\end{array}$ & $\begin{array}{l}\text { Residential } \\
\text { and Commercial }\end{array}$ & Total \\
\hline & \multicolumn{5}{|c|}{ ORIGIN: WEST VIRGINIA, SOUTHERN (Continued) } \\
\hline 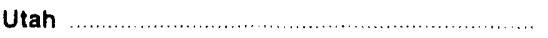 & - & 35 & - & - & 35 \\
\hline 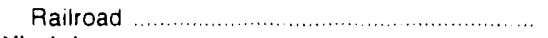 & - & 35 & - & - & 35 \\
\hline 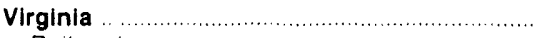 & 1,588 & - & 482 & 34 & 2,104 \\
\hline 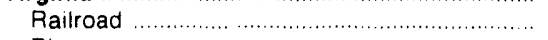 & 1.583 & - & 383 & 25 & 1,991 \\
\hline River & - & - & - & 1 & 1 \\
\hline 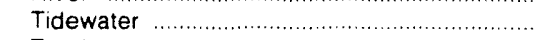 & 5 & - & 7 & - & 12 \\
\hline Truck & - & - & 92 & 8 & 100 \\
\hline 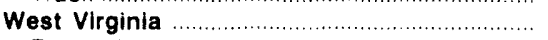 & 4,662 & 1,507 & 494 & 10 & 26,678 \\
\hline Railroad & 3,336 & 877 & 80 & - & 4,293 \\
\hline 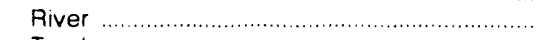 & 1,172 & 630 & 108 & - & 1.910 \\
\hline Truck & 154 & - & 263 & 5 & 422 \\
\hline 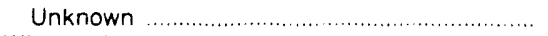 & - & - & 42 & 6 & 252 \\
\hline 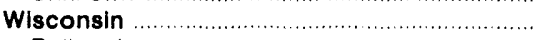 & 78 & - & 45 & - & 123 \\
\hline Railroad & 61 & - & - & - & 61 \\
\hline River & - & - & 26 & - & 26 \\
\hline 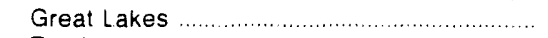 & 17 & - & 10 & - & 27 \\
\hline 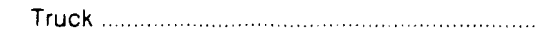 & - & - & 9 & - & 9 \\
\hline Unknown State & - & - & - & - & 1130 \\
\hline 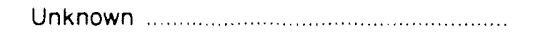 & - & - & - & - & 1130 \\
\hline State Total & 22,586 & 6,058 & 3,504 & 119 & 32,400 \\
\hline 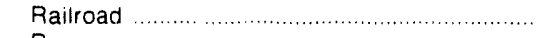 & 14.963 & 4,680 & 2.097 & 55 & 21,794 \\
\hline 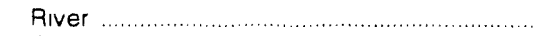 & 6.247 & 1,354 & 573 & 4 & 8,178 \\
\hline 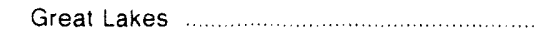 & 75 & 24 & 63 & 1 & 163 \\
\hline 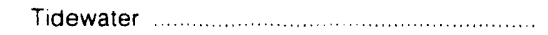 & 724 & - & 7 & - & 731 \\
\hline 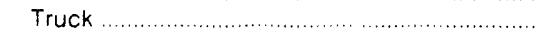 & 577 & * & 723 & 53 & 1.353 \\
\hline 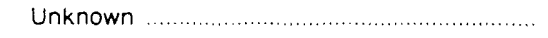 & - & - & 42 & 6 & 3182 \\
\hline
\end{tabular}

ORIGIN: WYOMING

\begin{tabular}{|c|c|}
\hline Alabama & 114 \\
\hline Railroad & 114 \\
\hline Arkansas . & 6,156 \\
\hline Railroad & 6,156 \\
\hline California . & \\
\hline Railroad ....................... & \\
\hline Colorado & 2,715 \\
\hline 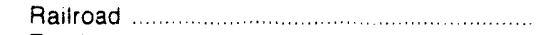 & 2,715 \\
\hline Truck & \\
\hline Georgla & 1,127 \\
\hline Railroad. & 1,127 \\
\hline Idaho ......... & \\
\hline Railroad ........................ & \\
\hline Truck & \\
\hline Illinois & 2,064 \\
\hline Railroad & 1,864 \\
\hline Great Lakes ................... & 20 \\
\hline Indiana & 5,400 \\
\hline 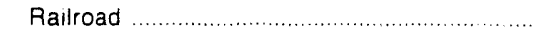 & 1,219 \\
\hline 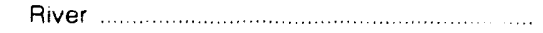 & 4,181 \\
\hline (1) & 6,935 \\
\hline 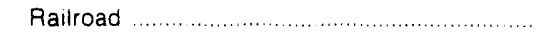 & 6,65 \\
\hline River & 28 \\
\hline Kansas & 6,46 \\
\hline 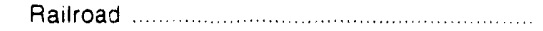 & 6.46 \\
\hline (1) & 18 \\
\hline … & 183 \\
\hline , & 4,183 \\
\hline 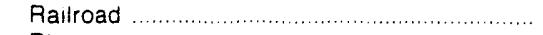 & 1,849 \\
\hline 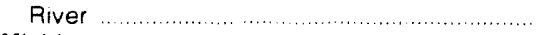 & 2.334 \\
\hline$\ldots \ldots \ldots \ldots \ldots \ldots \ldots$ & 1,150 \\
\hline 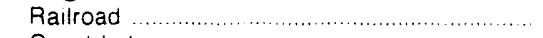 & 1,117 \\
\hline Great Lakes & 3 \\
\hline 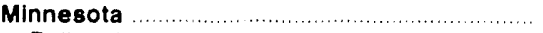 & 3,58 \\
\hline 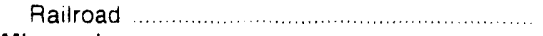 & 3,58 \\
\hline 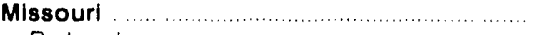 & 3,85 \\
\hline Raılroad & 3.85 \\
\hline
\end{tabular}

See footnotes at end of table 

Table 33. Domestic Distribution of U.S. Coal by Origin State, Consumer, Destination
and Method of Transportation, January-June 1991 (Continued) (Thousand Short Tons)

\begin{tabular}{|c|c|c|c|c|c|}
\hline $\begin{array}{l}\text { State of Destination } \\
\text { by Method of Transportation }\end{array}$ & $\begin{array}{l}\text { Electric } \\
\text { Utilities }\end{array}$ & $\begin{array}{l}\text { Coke } \\
\text { Plants }\end{array}$ & $\begin{array}{c}\text { Industrial } \\
\text { Plants } \\
\text { (Except Coke) }\end{array}$ & $\begin{array}{c}\text { Residential } \\
\text { and Commercial }\end{array}$ & Total \\
\hline & \multicolumn{5}{|c|}{ ORIGIN: WYOMING (Continued) } \\
\hline Montana & - & - & 66 & * & 66 \\
\hline $\begin{array}{l}\text { Railroad } \\
\text { Truck }\end{array}$ & - & - & 66 & $=$ & 66 \\
\hline $\begin{array}{l}\text { Truck } \\
\text { Nebraska }\end{array}$ & - & - & - & · & \\
\hline $\begin{array}{l}\text { Nebraska } \\
\text { Railroad }\end{array}$ & 4,206 & - & - & - & 4,206 \\
\hline $\begin{array}{l}\text { Railroad } \\
\text { Nevada }\end{array}$ & 4,206 & - & - & - & 4,206 \\
\hline & 193 & - & - & - & 193 \\
\hline Raulroad & 193 & - & - & - & 193 \\
\hline New York & 9 & - & - & - & 9 \\
\hline 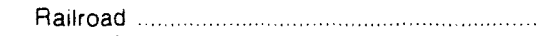 & 9 & - & - & - & 9 \\
\hline North Dakota & - & - & - & * & * \\
\hline Truck & - & - & - & . & • \\
\hline Ohio & 33 & - & - & - & 33 \\
\hline Ralload & 33 & - & - & - & 33 \\
\hline Oklahoma & 7,734 & - & 33 & - & 7,767 \\
\hline Raulroad & 7,734 & - & 33 & - & 7,767 \\
\hline (2) & 1,045 & - & 18 & - & 1,063 \\
\hline Ralroad & 1,045 & - & 18 & - & 1,063 \\
\hline South Dakota & 127 & - & 98 & 2 & 227 \\
\hline Truck & 127 & - & 98 & 2 & 227 \\
\hline Tennessee & 639 & - & - & - & 639 \\
\hline Railroad & 639 & - & - & - & 639 \\
\hline 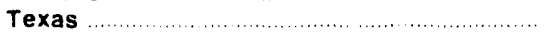 & 17,467 & - & 109 & * & 17,576 \\
\hline Railroad & 17,467 & - & 109 & - & 17,576 \\
\hline 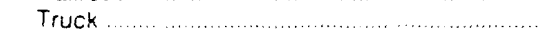 & - & - & - & - & \\
\hline Utah & 214 & - & 2 & - & 216 \\
\hline 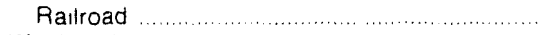 & 214 & - & 2 & - & 216 \\
\hline Washington & - & - & 23 & 1 & 24 \\
\hline Railroad & - & - & 23 & 1 & 24 \\
\hline Wisconsin & 5,975 & - & 166 & - & 6,141 \\
\hline Railroad & 5.959 & - & 166 & - & 6.125 \\
\hline 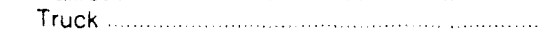 & 16 & - & - & - & 16 \\
\hline Wyoming & 11,179 & - & 787 & 63 & 12,029 \\
\hline Railroad & 5,526 & - & 615 & 8 & 6.148 \\
\hline 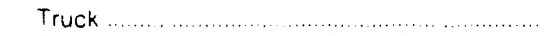 & 865 & - & 162 & 55 & 1,082 \\
\hline Tramway, Conveyor, and Slurry Pipeline ........ & 4,788 & - & - & - & 4,788 \\
\hline Unknown & - & - & 9 & 1 & 10 \\
\hline Unknown State & - & - & - & - & 19 \\
\hline Unknown & - & - & - & - & 19 \\
\hline State Total & 92,743 & - & 2,195 & 126 & 195,073 \\
\hline Railroad. & 79.917 & - & 1,846 & 65 & 81,829 \\
\hline …….................... & 6.796 & - & - & - & 6.796 \\
\hline Great Lakes & 233 & - & - & - & 233 \\
\hline 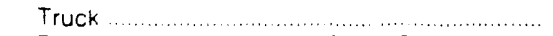 & 1.009 & - & 339 & 60 & 1.408 \\
\hline Tramway, Conveyor, and Slurry Pipeline ........ & 4,788 & - & - & - & 4,788 \\
\hline \multirow[t]{2}{*}{ Unknown } & - & - & 9 & 1 & 120 \\
\hline & \multicolumn{5}{|c|}{ ORIGIN: U.S. TOTAL } \\
\hline … & 11,599 & 1,139 & 1,188 & 10 & 13,937 \\
\hline Ratroad & 4,118 & 650 & 421 & 6 & 5.195 \\
\hline River & 2,937 & - & 228 & - & 3,165 \\
\hline Trucl & 3.689 & 489 & 481 & 4 & 4.663 \\
\hline Tramway. Conveyor, and Slurry Pipeline ........ & 855 & - & - & - & 855 \\
\hline 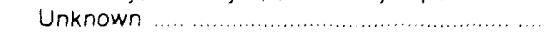 & - & - & 59 & - & 59 \\
\hline Alaska & 156 & - & - & 256 & 412 \\
\hline Raltroad & 76 & - & - & 252 & 328 \\
\hline Truck & 80 & - & - & 4 & 84 \\
\hline 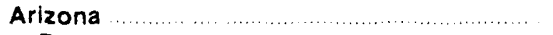 & 8,133 & - & 369 & * & 8,502 \\
\hline Railroad & 8,132 & - & 360 & - & 8.492 \\
\hline Truck & 1 & - & 9 & - & 10 \\
\hline Arkansas & 6,156 & - & 110 & • & 6,265 \\
\hline Railroad & 6.156 & - & 54 & - & 6,210 \\
\hline 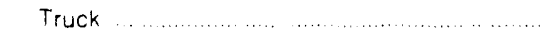 & - & - & 56 & . & 56 \\
\hline 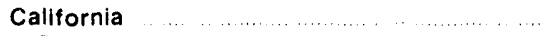 & - & - & 1,388 & 43 & 1,431 \\
\hline Raliroad .... & - & - & 1.379 & 43 & 1.422 \\
\hline Truck $\ldots \ldots \ldots$ & - & - & 9 & - & 9 \\
\hline
\end{tabular}

See footnotes at end of table. 
Table 33. Domestic Distribution of U.S. Coal by Origin State, Consumer, Destination and Method of Transportation, January-June 1991 (Continued) (Thousand Short Tons)

\begin{tabular}{|c|c|c|c|c|c|}
\hline $\begin{array}{l}\text { State of Destination } \\
\text { by Method of Transportation }\end{array}$ & $\begin{array}{l}\text { Electric } \\
\text { Utillities }\end{array}$ & $\begin{array}{l}\text { Coke } \\
\text { Plants }\end{array}$ & $\begin{array}{c}\text { Industrial } \\
\text { Plants } \\
\text { (Except Coke) }\end{array}$ & $\begin{array}{c}\text { Residential } \\
\text { and Commercial }\end{array}$ & Total \\
\hline & \multicolumn{5}{|c|}{ ORIGIN: U.S. TOTAL (Continued) } \\
\hline Colorado. & 7,613 & - & 329 & 28 & 27,970 \\
\hline Railroad & 5,432 & - & 232 & 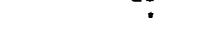 & 5,664 \\
\hline Truck … & 2,182 & - & 97 & 27 & 2,306 \\
\hline 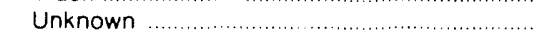 & - & - & - & 1 & 21 \\
\hline 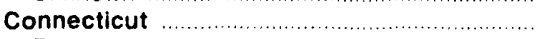 & 523 & - & - & 3 & 527 \\
\hline 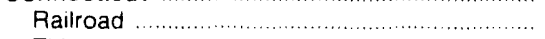 & 191 & - & - & . & 191 \\
\hline 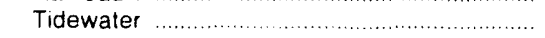 & 332 & - & - & - & 332 \\
\hline 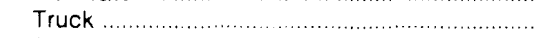 & - & - & - & 3 & 3 \\
\hline Delaware & 1,010 & - & 91 & 4 & 1,105 \\
\hline Raitroad & 1,010 & - & 88 & 4 & 1,102 \\
\hline 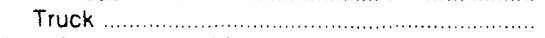 & 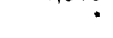 & - & 3 & $\cdot$ & 3 \\
\hline District of Columbia & - & - & - & 41 & 41 \\
\hline 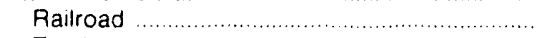 & - & - & - & 35 & 35 \\
\hline 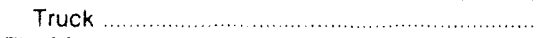 & - & - & - & 6 & 6 \\
\hline Florida & $11,0,17$ & - & 367 & 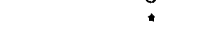 & 11,384 \\
\hline Railroad & 5,126 & - & 364 & - & 5.489 \\
\hline River & 4,537 & - & - & - & 4,537 \\
\hline 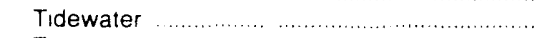 & 1,354 & - & - & - & 1.354 \\
\hline 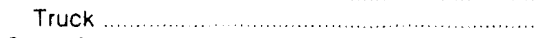 & 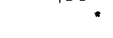 & - & 4 & * & 4 \\
\hline 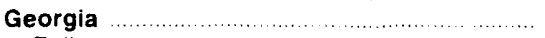 & 11,629 & - & 1,014 & 4 & 12,647 \\
\hline . & 9.799 & - & 928 & 4 & 10,732 \\
\hline 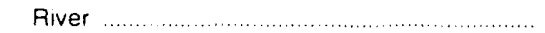 & 1.829 & - & - & - & 829 \\
\hline Truck & 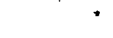 & - & 86 & • & 86 \\
\hline Idaho & - & - & 243 & 36 & 279 \\
\hline . & - & - & 145 & 1 & 145 \\
\hline 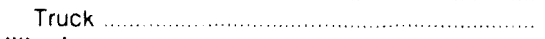 & - & - & 99 & 35 & 134 \\
\hline 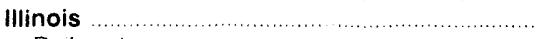 & 13,961 & 766 & 2,249 & 102 & 17,077 \\
\hline 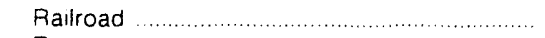 & 9,027 & 324 & 802 & 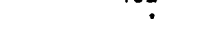 & 10,153 \\
\hline 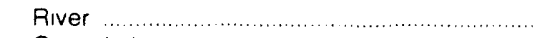 & 1,737 & 442 & 430 & - & 2,609 \\
\hline 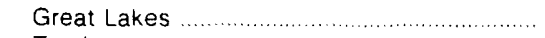 & 211 & - & - & - & 211 \\
\hline 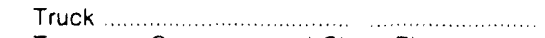 & 1.749 & - & 1.005 & 99 & 2,853 \\
\hline Tramway, Conveyor, and Slurry Pipeline ....... & 1,237 & - & - & - & 1,237 \\
\hline Unknown & - & - & 12 & 2 & 14 \\
\hline Indiana & 21,602 & 3,090 & 2,040 & 245 & 26,976 \\
\hline Railroad $\ldots \ldots \ldots \ldots$ & 12,574 & 2,983 & 1,210 & 11 & 16,778 \\
\hline 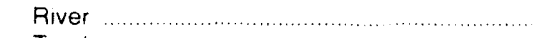 & 6,683 & 106 & 158 & - & 6.947 \\
\hline 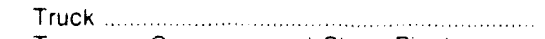 & 1,992 & 1 & 661 & 233 & 2.887 \\
\hline Tramway, Conveyor, and Slurry Pipelıne ....... & 352 & - & - & - & 352 \\
\hline 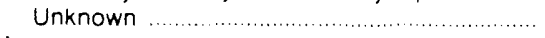 & - & - & 11 & 1 & 11 \\
\hline 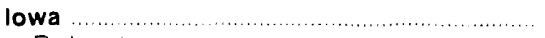 & 8,386 & - & 459 & 152 & 8,996 \\
\hline 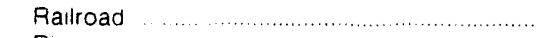 & 7,074 & - & 193 & 13 & 7,280 \\
\hline 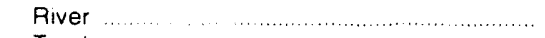 & 1,130 & - & 266 & 138 & 1.534 \\
\hline 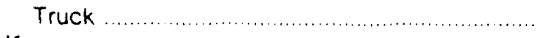 & 182 & - & - & 1 & 183 \\
\hline Kansas & 6,907 & - & 41 & $\cdot$ & 6,949 \\
\hline 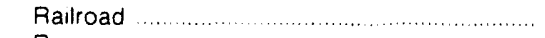 & 6,849 & - & $\because$ & - & 6.849 \\
\hline 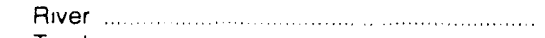 & - & - & 8 & - & 8 \\
\hline Truck & 58 & - & 33 & $\cdot$ & 91 \\
\hline 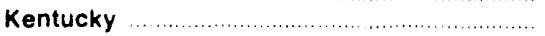 & 13,711 & 405 & 931 & 102 & 215,148 \\
\hline 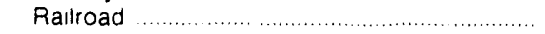 & 4,986 & 353 & 366 & 18 & 5,724 \\
\hline … & 4,257 & 48 & 42 & $\cdot$ & 4,348 \\
\hline 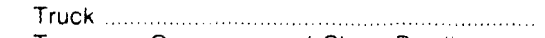 & 3,192 & 4 & 514 & 47 & 3,757 \\
\hline Tramway, Conveyor, and Slurry Pipeline ....... & 1,275 & - & - & - & 1,275 \\
\hline Unknown & - & - & 8 & 37 & 246 \\
\hline Louisiana & 5,792 & - & 256 & - & 6,048 \\
\hline Railroad & 1,849 & - & 194 & - & 2,043 \\
\hline River & 2,652 & - & 62 & - & 2,714 \\
\hline Tidewater & 4 & - & - & - & 4 \\
\hline Truck & 177 & - & - & - & 177 \\
\hline Tramway, Conveyor, and Slurry Pipeline ....... & 1.111 & - & - & - & 1,111 \\
\hline Maine & - & - & 10 & 3 & 12 \\
\hline Raltroad & - & - & 10 & 1 & 11 \\
\hline Truck ..... & - & - & - & 2 & 2 \\
\hline$\ldots \ldots \ldots \ldots \ldots \ldots \ldots \ldots$ & 4,529 & 267 & 614 & 13 & 5,422 \\
\hline Railroad & 3,902 & 152 & 467 & . & 4.522 \\
\hline River $\ldots \ldots \ldots \ldots$ & - & 78 & - & - & 78 \\
\hline 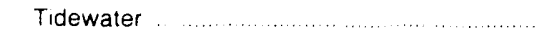 & 530 & 36 & - & - & 567 \\
\hline Truck & 96 & - & 135 & 12 & 244 \\
\hline Unknown & - & - & 12 & . & 12 \\
\hline
\end{tabular}

See footnotes at end of table 

Table 33. Domestic Distribution of U.S. Coal by Origin State, Consumer, Destination
and Method of Transportation, January-June 1991 (Continued) (Thousand Short Tons)

\begin{tabular}{|c|c|c|c|c|c|}
\hline $\begin{array}{l}\text { State of Destination } \\
\text { by Method of Transportation }\end{array}$ & $\begin{array}{l}\text { Electric } \\
\text { Utillties }\end{array}$ & $\begin{array}{l}\text { Coke } \\
\text { Plants }\end{array}$ & $\begin{array}{c}\text { Industrial } \\
\text { Plants } \\
\text { (Except Coke) }\end{array}$ & $\begin{array}{l}\text { Residentlal } \\
\text { and Commercial }\end{array}$ & Total \\
\hline$\ldots$ & \multicolumn{5}{|c|}{ ORIGIN: U.S. TOTAL (Continued) } \\
\hline Massachusetts & 1,537 & - & 27 & 8 & 1,572 \\
\hline Railroad & 420 & - & 22 & 2 & 444 \\
\hline 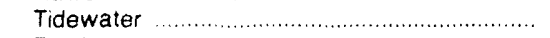 & 1,096 & - & - & - & 1.096 \\
\hline 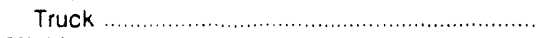 & 21 & - & 5 & 6 & 32 \\
\hline Michigan & 13.352 & 221 & 1,407 & 92 & 15,072 \\
\hline 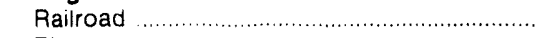 & 9,994 & 164 & 703 & 74 & 10,935 \\
\hline 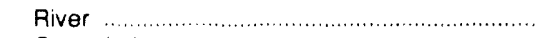 & - & - & 8 & - & 8 \\
\hline 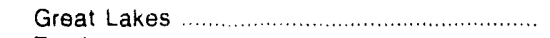 & 3,358 & 53 & 470 & 2 & 3,883 \\
\hline 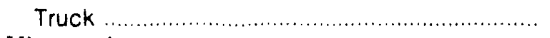 & $\dot{*}$ & 4 & 226 & 16 & 247 \\
\hline 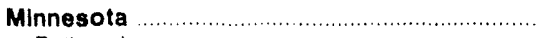 & 8,541 & - & 219 & 58 & 8,817 \\
\hline 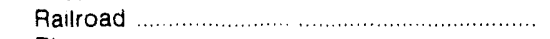 & 8,473 & - & 168 & 58 & 8,699 \\
\hline 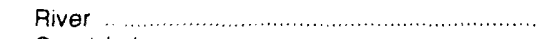 & 17 & - & 31 & - & 48 \\
\hline 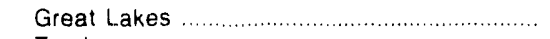 & 20 & - & 18 & - & 39 \\
\hline 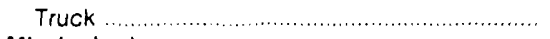 & 31 & - & 1 & $\cdot$ & 31 \\
\hline 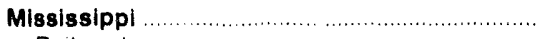 & 1,823 & - & 72 & - & 1,895 \\
\hline 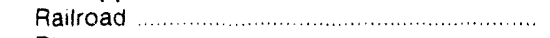 & 1.073 & - & * & - & 1,074 \\
\hline 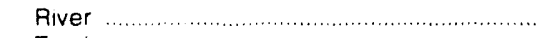 & 750 & - & 60 & - & 810 \\
\hline Truck & - & - & 11 & - & 11 \\
\hline 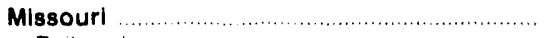 & 11,409 & - & 533 & 136 & 12,077 \\
\hline (1) & 8,403 & - & 6 & - & 8,409 \\
\hline 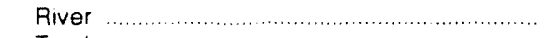 & 1,986 & - & 174 & 2 & 2,162 \\
\hline 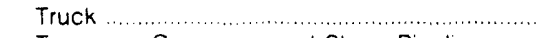 & 198 & - & 353 & 134 & 685 \\
\hline Tramway, Conveyor, and Slurry Pipeline ....... & 821 & - & - & - & 821 \\
\hline Montana & 4,982 & - & 130 & 21 & 5,132 \\
\hline 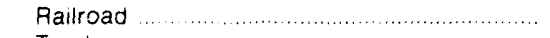 & 460 & - & 91 & - & 551 \\
\hline 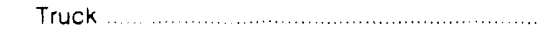 & 125 & - & 38 & 21 & 184 \\
\hline Tramway, Conveyor, and Slurry Pipeline ....... & 4,397 & - & - & - & 4,397 \\
\hline Unknown & - & - & $\dot{8}$ & $\overline{-}$ & 争 \\
\hline Nebraska $\ldots \ldots$ & 4,206 & - & 65 & 7 & 4,278 \\
\hline Rallroad $\ldots \ldots \ldots$ & 4,206 & - & 65 & $?$ & 4,278 \\
\hline Nevada $\ldots \ldots \ldots \ldots \ldots$ & 4,190 & - & 113 & 1 & 4,305 \\
\hline Railroad $\ldots . . . \ldots \ldots$ & 1,615 & - & 92 & - & 1,708 \\
\hline 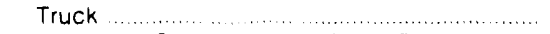 & - & - & 21 & 1 & 22 \\
\hline Tramway. Conveyor, and Slurry Pipeline ....... & 2.575 & - & - & 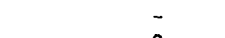 & 2,575 \\
\hline 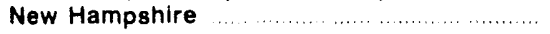 & 703 & - & 1 & 6 & 710 \\
\hline Railroad & 474 & - & 1 & 2 & 476 \\
\hline 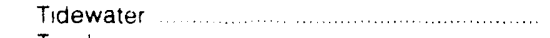 & 229 & - & - & - & 229 \\
\hline 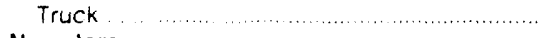 & - & - & - & 5 & 5 \\
\hline New Jersey $\ldots \ldots \ldots$ & 831 & - & 140 & 4 & 975 \\
\hline Railroad $\ldots \ldots \ldots$ & 399 & - & 136 & $\cdot$ & 536 \\
\hline River $\ldots \ldots$ & 41 & - & - & - & 41 \\
\hline 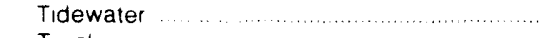 & 391 & - & - & 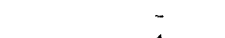 & 391 \\
\hline 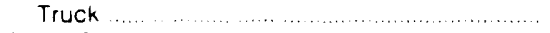 & - & - & 4 & 4 & 8 \\
\hline 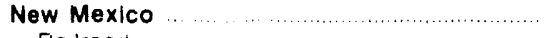 & 5,774 & - & 22 & 4 & 5,799 \\
\hline 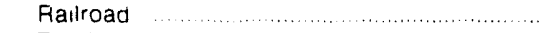 & 410 & - & - & - & 410 \\
\hline . & 8 & - & 22 & 3 & 33 \\
\hline Tramway, Conveyor, and Siurry Pipeline ....... & 5.355 & - & - & - & 5,355 \\
\hline 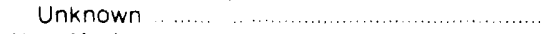 & - & - & - & $\cdot$ & $\cdot$ \\
\hline New York & 5,064 & 471 & 829 & 128 & 6,492 \\
\hline Ralload $\ldots \ldots \ldots$ & 4,514 & 465 & 641 & 26 & 5.646 \\
\hline River & 228 & - & 68 & 9 & 306 \\
\hline Great Lakes & 6 & - & - & 1 & 7 \\
\hline 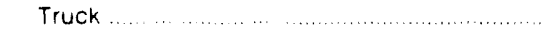 & 317 & 6 & 120 & 91 & 534 \\
\hline North Carolina & 9,211 & - & 1,238 & 47 & 10,495 \\
\hline$\ldots \ldots \ldots \ldots \ldots, \ldots \ldots \ldots$ & $9,2 \uparrow 1$ & - & 1.040 & 45 & 10,295 \\
\hline 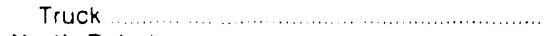 & - & - & 198 & 2 & 200 \\
\hline 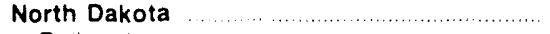 & 10,287 & - & 3,109 & 74 & 13,470 \\
\hline Railroad $\ldots \ldots \ldots \ldots \ldots$ & 913 & - & 140 & 16 & 1,069 \\
\hline 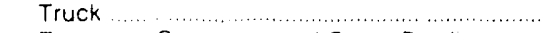 & 1,848 & - & 11 & 36 & 1,895 \\
\hline Tramway, Conveyor, and Slurry Pipeline ....... & 7.526 & - & 2.958 & 22 & 10.506 \\
\hline Unknown & - & - & - & 1 & 1 \\
\hline Ohio $\ldots \ldots \ldots$ & 25,346 & 1,732 & 2,342 & 226 & 29,647 \\
\hline 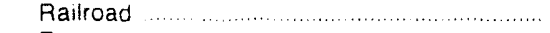 & 4,252 & 1,561 & 770 & 7 & 6.590 \\
\hline 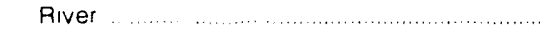 & 12,522 & 163 & 65 & * & 12,750 \\
\hline Great Lakes & 144 & - & 66 & - & 210 \\
\hline 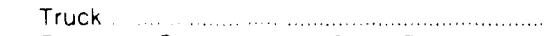 & 3,960 & 8 & 1,437 & 219 & 5,624 \\
\hline Tramway, Conveyor. and Slurry Pipeline ....... & 4,468 & - & - & - & 4,468 \\
\hline Unknown & - & - & 5 & $\cdot$ & 5 \\
\hline
\end{tabular}

See footnotes at end of table 
Table 33. Domestic Distribution of U.S. Coal by Origin State, Consumer, Destination and Method of Transportation, January-June 1991 (Continued) (Thousand Short Tons)

\begin{tabular}{|c|c|c|c|c|c|}
\hline $\begin{array}{l}\text { State of Destination } \\
\text { by Method of Transportation }\end{array}$ & $\begin{array}{l}\text { Electric } \\
\text { Utilitios }\end{array}$ & $\begin{array}{l}\text { Coke } \\
\text { Plants }\end{array}$ & $\begin{array}{c}\begin{array}{c}\text { Industrial } \\
\text { Plants } \\
\text { (Except Coke) }\end{array}\end{array}$ & $\begin{array}{l}\text { Residential } \\
\text { and Commercial }\end{array}$ & Total \\
\hline & \multicolumn{5}{|c|}{ ORIGIN: U.S. TOTAL (Continued) } \\
\hline Oklahoma & 8,176 & - & 315 & * & 8,492 \\
\hline Railroad & 7,747 & - & 90 & - & 7.837 \\
\hline River & - & - & 9 & - & 9 \\
\hline 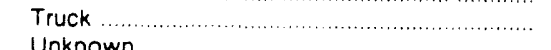 & 430 & - & 217 & : & 646 \\
\hline Unknown & - & - & - & : & - \\
\hline 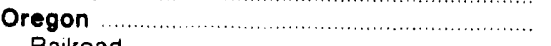 & 1,045 & - & 18 & 1 & 1,063 \\
\hline Railroad & 1,045 & - & 18 & - & 1,063 \\
\hline $\begin{array}{l}\text { Truck } \\
\text { Pennsylvania }\end{array}$ & 210 & 50 & - & 1 & 1 \\
\hline 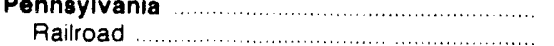 & 21,104 & 3,007 & 1,719 & 675 & 26,505 \\
\hline River & $\begin{array}{l}6,309 \\
5,606\end{array}$ & $\begin{array}{l}1,021 \\
1,934\end{array}$ & 243 & 39 & 7,612 \\
\hline Great Lakes & 22 & 1,934 & 136 & - & 7,676 \\
\hline 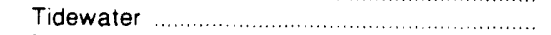 & 34 & - & - & - & 22 \\
\hline Truck & 4,195 & 52 & - & - & 34 \\
\hline Tramway, Conveyor, and Slurry Pipeline ........ & 4,939 & - & 1.314 & 633 & 6,194 \\
\hline 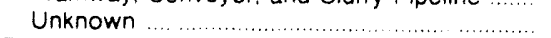 & - & - & - & - & 4,939 \\
\hline Rhode Island & - & - & 26 & 3 & 29 \\
\hline Railroad & - & - & - & 1 & $!$ \\
\hline …………… & - & - & - & i & $\dot{1}$ \\
\hline South Carulina & 4,297 & - & 1,058 & 16 & 5,371 \\
\hline Railroad & 4,294 & - & 971 & 15 & 5,280 \\
\hline Truck & 2 & - & 87 & 1 & 90 \\
\hline South Dakota & 1,406 & - & 103 & 2 & 1,510 \\
\hline 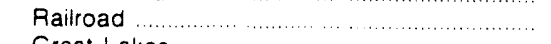 & 1.278 & - & • & - & 1,278 \\
\hline Great Lakes & - & - & 5 & - & 5 \\
\hline Truck & 127 & - & 98 & 2 & 227 \\
\hline (2) & 11,264 & - & 1,776 & 128 & 13,167 \\
\hline 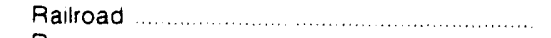 & 4,740 & - & 1.202 & 56 & 5,997 \\
\hline 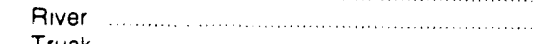 & 6.524 & - & 37 & 33 & 6.594 \\
\hline Truck & • & - & 536 & 39 & 575 \\
\hline 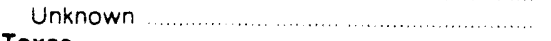 & - & - & - & • & \\
\hline Texas & 43,216 & - & 1,423 & 6 & 44,645 \\
\hline 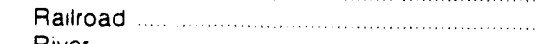 & 28,845 & - & 647 & 6 & 29,498 \\
\hline River & - & - & 3 & - & 3 \\
\hline Truck & 6,403 & - & 118 & • & 6,521 \\
\hline Tramway. Conveyor, and Slurry Pipelıne & 7,968 & - & 655 & - & 8,623 \\
\hline Utah & 6,891 & 669 & 279 & 161 & 8,001 \\
\hline Railroad $\ldots \ldots \ldots \ldots$ & 3,235 & 669 & 2 & - & 3,907 \\
\hline Truck ........ & 2.462 & - & 276 & 160 & 2,898 \\
\hline Tramway, Conveyo:, and Slurry Pipeline ....... & 1.194 & - & - & - & 1.194 \\
\hline Unknown & - & - & 1 & 2 & 3 \\
\hline 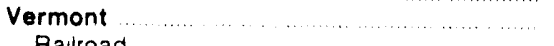 & - & - & 7 & 2 & 9 \\
\hline 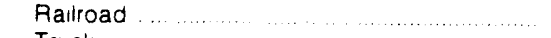 & - & - & 7 & : & 7 \\
\hline 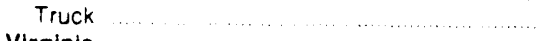 & - & - & - & 2 & 2 \\
\hline 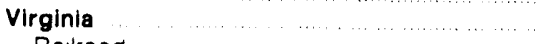 & 4,658 & 480 & 1,830 & 64 & 27,031 \\
\hline Rallroad $\ldots$ & 4,507 & - & 1.402 & 48 & 5.956 \\
\hline River & - & - & - & 1 & 1 \\
\hline 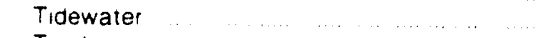 & 5 & - & 217 & - & 222 \\
\hline Truck & 146 & - & 199 & 12 & 356 \\
\hline Tramway Conveyor and Slurry Pipeline ..... & - & 480 & - & - & 480 \\
\hline Unknown ................... & - & - & 13 & 3 & 216 \\
\hline Washington & 2,193 & - & 81 & 60 & 2,334 \\
\hline$\ldots \ldots \ldots$ & 2,193 & - & 81 & 60 & 2.333 \\
\hline$\ldots \ldots \ldots$ & & - & . & 1 & 1 \\
\hline West Virginia & 14,578 & 2,026 & 1,226 & 132 & 217,967 \\
\hline$\ldots \ldots+\ldots$ & 6.615 & 877 & 288 & 7 & 7,789 \\
\hline River & 3,390 & 1,149 & 346 & - & 4,885 \\
\hline Truck & 2,207 & - & 541 & 118 & 2,866 \\
\hline Tramway. Conveyor and Slurry Pipeline & 2.366 & - & - & - & 2.366 \\
\hline Unknown & & - & 51 & 7 & 262 \\
\hline$\ldots \ldots$ & 9,816 & - & 738 & 9 & 10,564 \\
\hline$\ldots \ldots \ldots$ & 8,972 & - & 400 & • & 9.372 \\
\hline River & 731 & - & 39 & 9 & 779 \\
\hline 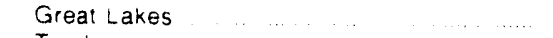 & 98 & - & 262 & - & 360 \\
\hline 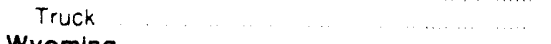 & 16 & - & 37 & - & 53 \\
\hline$\ldots \ldots \ldots \ldots \ldots$ & 11,179 & - & 856 & 63 & 12,098 \\
\hline$\ldots \quad \ldots \quad \ldots$ & 5,526 & - & 649 & 8 & 6.183 \\
\hline Truck & 865 & - & 197 & 55 & 1.117 \\
\hline
\end{tabular}

See footnotes at end of table 

Table 33. Domestic Distribution of U.S. Coal by Origin State, Consumer, Destination
and Method of Transportation, January-June 1991 (Continued) (Thousand Short Tons)

\begin{tabular}{|c|c|c|c|c|c|}
\hline $\begin{array}{l}\text { State of Destination } \\
\text { by Method of Transportation }\end{array}$ & $\begin{array}{l}\text { Electric } \\
\text { Utilities }\end{array}$ & $\begin{array}{l}\text { Coke } \\
\text { Plants }\end{array}$ & $\begin{array}{c}\text { Industrial } \\
\text { Plants } \\
\text { (Except Coke) }\end{array}$ & $\begin{array}{l}\text { Residential } \\
\text { and Commercial }\end{array}$ & rotal \\
\hline & \multicolumn{5}{|c|}{ ORIGIN: U.S. TOTAL (Continued) } \\
\hline \multicolumn{6}{|l|}{ Wyoming } \\
\hline Tramway, Conveyor, and Slurry Pipeline ....... & 4,788 & - & - & - & 4,788 \\
\hline Unknown & - & - & 9 & 1 & 10 \\
\hline Unknown State & - & - & - & - & 11,834 \\
\hline Unknown & - & - & - & - & 11,834 \\
\hline U.S. Total & 379,800 & 14,272 & 33,374 & 3,173 & ${ }^{3} 432,457$ \\
\hline (2) & 226,421 & 9,220 & 17,088 & 867 & 253,596 \\
\hline 대 & 57.558 & 3,921 & 2,171 & 192 & 63,841 \\
\hline Great Lakes & 3,858 & 53 & 822 & 3 & 4,736 \\
\hline 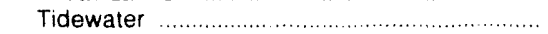 & 3,974 & 36 & 217 & - & 4,228 \\
\hline Truck & 36,762 & 563 & 9,256 & 2,032 & 48,613 \\
\hline Tramway, Conveyor, and Siurry Pipeline ....... & 51,227 & 480 & 3,613 & 22 & 55,342 \\
\hline Unknown & - & - & 207 & 57 & 32,103 \\
\hline
\end{tabular}

1 Includes distribution to unknown consumers.

2 Includes distribution to the transportation sector.

3 includes distribution to both the transportation sector and unknown consumers.

Quantity is less than 500 short tons or percent is less than .05

Note: Total may not equal sum of components because of independent rounding.

Source: Energy Information Administration. Form ElA-6, "Coal Distribution Report." 

Table 34. Domestic Distribution of U.S. Coal by Destination State, Consumer,
Origin and Method of Transportation, January-June 1991 (Thousand Short Tons)

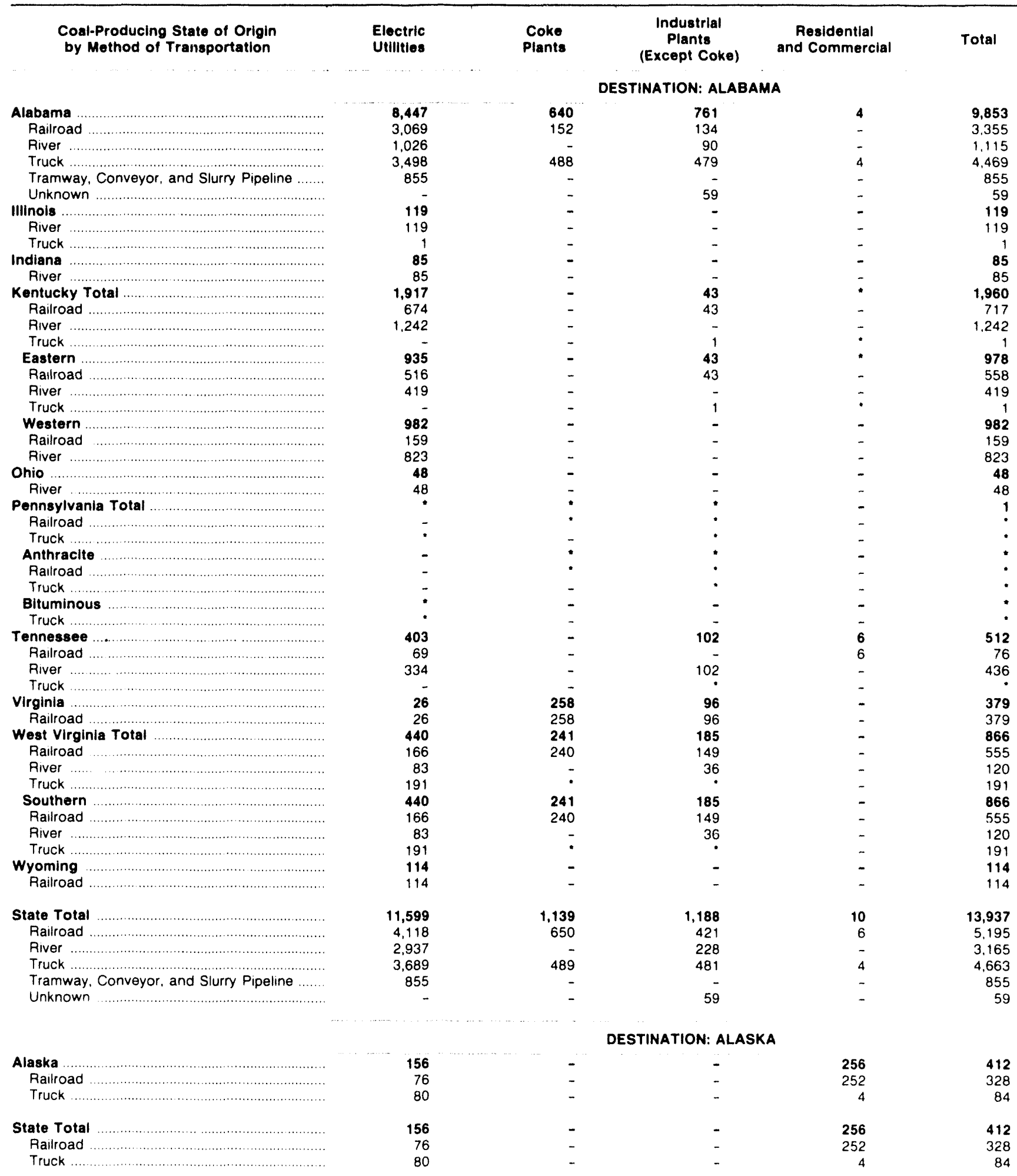

See footnotes at end of table. 
Table 34. Domestic Distribution of U.S. Coal by Destination State, Consumer, Origin and Method of Transportation, January-June 1991 (Continued) (Thousand Short Tons)

\begin{tabular}{|c|c|c|c|c|c|}
\hline $\begin{array}{l}\text { Coal-Producing State of Origin } \\
\text { by Method of Transportation }\end{array}$ & $\begin{array}{l}\text { Electric } \\
\text { Utllities }\end{array}$ & $\begin{array}{l}\text { Coke } \\
\text { Plants }\end{array}$ & $\begin{array}{c}\text { Induetrial } \\
\text { Plante } \\
\text { (Except Coke) }\end{array}$ & $\begin{array}{l}\text { Residential } \\
\text { and Commercial }\end{array}$ & Total \\
\hline & \multicolumn{5}{|c|}{ DESTINATION: ARIZONA } \\
\hline$\ldots \ldots \ldots \ldots \ldots \ldots \ldots$ & 3,787 & - & - & - & 3,787 \\
\hline 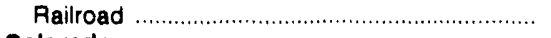 & 3,787 & - & - & - & 3,787 \\
\hline Colorado & 350 & - & 70 & - & 420 \\
\hline 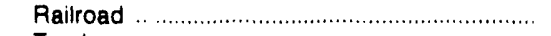 & 350 & - & 60 & - & 411 \\
\hline 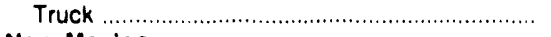 & - & - & 9 & - & 9 \\
\hline Now Moxjco & 3,995 & - & 243 & - & 4,239 \\
\hline $\begin{array}{l}\text { Railroad } \\
\text { Truck }\end{array}$ & 3,995 & - & 243 & $\overline{-}$ & 4,236 \\
\hline $\begin{array}{r}\text { Truck } \\
\text { Ponnaylvania Total }\end{array}$ & - & - & $\overline{-}$ & $\dot{0}$ & $\dot{*}$ \\
\hline $\begin{array}{l}\text { Pennsylvania Total } \\
\text { Raiiroad }\end{array}$ & - & - & : & " & : \\
\hline & - & - & " & $\overline{-}$ & $\dot{0}$ \\
\hline $\begin{array}{l}\text { Truck } \\
\text { Anthracite }\end{array}$ & - & $\overline{-}$ & $\dot{\square}$ & * & 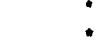 \\
\hline $\begin{array}{c}\text { Anthracite } \\
\text { Railroad }\end{array}$ & $\overline{-}$ & - & & & : \\
\hline $\begin{array}{l}\text { Railroad } \\
\text { Truck }\end{array}$ & - & - & - & $\overline{-}$ & . \\
\hline $\begin{array}{c}\text { Truck } \\
\text { Utah }\end{array}$ & $\overline{1}$ & & & & 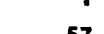 \\
\hline $\begin{array}{l}\text { Utah } \\
\text { Railroad }\end{array}$ & $\begin{array}{l}1 \\
-\end{array}$ & - & 56 & - & 57 \\
\hline $\begin{array}{l}\text { Railroad } \\
\text { Truck }\end{array}$ & $\bar{i}$ & - & 56 & - & 56 \\
\hline & 1 & - & - & - & 1 \\
\hline 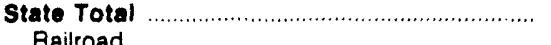 & 8,133 & $=$ & 369 & • & 8,502 \\
\hline 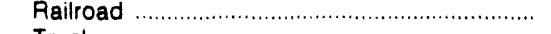 & 8,132 & - & 360 & - & 8,492 \\
\hline \multirow[t]{2}{*}{ 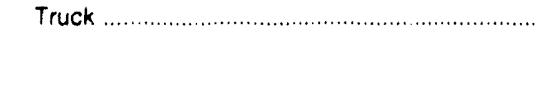 } & 1 & - & 9 & $\cdot$ & 10 \\
\hline & \multicolumn{5}{|c|}{ DESTINATION: ARKANSAS } \\
\hline Alabama & - & - & 3 & - & 3 \\
\hline 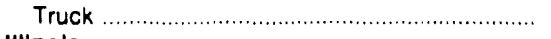 & - & - & 3 & - & 3 \\
\hline Illinols & - & - & 54 & - & 54 \\
\hline Railroad & - & - & 54 & - & 54 \\
\hline Kentucky Total & - & - & 5 & - & 5 \\
\hline 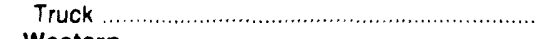 & - & - & 5 & - & 5 \\
\hline 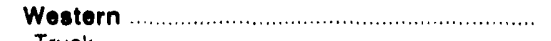 & - & - & 5 & - & 5 \\
\hline Truck & - & - & 5 & $\overline{-}$ & 5 \\
\hline Oklahoma & - & - & 46 & ". & 46 \\
\hline Truck & - & - & 46 & $\bullet$ & 46 \\
\hline 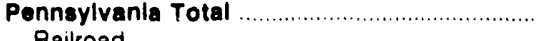 & - & - & 1 & - & 1 \\
\hline 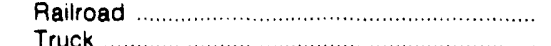 & - & - & $\dot{1}$ & - & 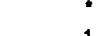 \\
\hline $\begin{array}{l}\text { Truck } \\
\text { Anthracite }\end{array}$ & - & $\overline{-}$ & 1 & - & 1 \\
\hline $\begin{array}{c}\text { Anthracito } \\
\text { Railroad }\end{array}$ & - & $\begin{array}{l}- \\
-\end{array}$ & 1 & - & $!$ \\
\hline $\begin{array}{l}\text { Railroad } \\
\text { Truck }\end{array}$ & - & - & $\dot{4}$ & - & 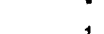 \\
\hline $\begin{array}{l}\text { Truck } \\
\text { Wyoming }\end{array}$ & $6,15 \overline{6}$ & - & 1 & - & $\begin{array}{r}1 \\
0.150\end{array}$ \\
\hline $\begin{array}{l}\text { Wyoming } \\
\text { Railroad }\end{array}$ & $\begin{array}{l}6,156 \\
6,156\end{array}$ & - & - & - & $\begin{array}{l}6,156 \\
6,156\end{array}$ \\
\hline & & & - & - & 6,156 \\
\hline State Total & 6,156 & - & 110 & - & 6,265 \\
\hline Railroad & 6,156 & - & 54 & - & 6,210 \\
\hline \multirow[t]{2}{*}{ Truck } & - & - & 56 & $\bullet$ & 56 \\
\hline & \multicolumn{5}{|c|}{ DESTINATION: CALIFORNIA } \\
\hline 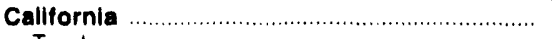 & - & - & 8 & - & 8 \\
\hline 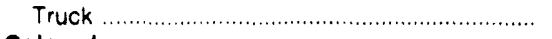 & - & - & 8 & - & 8 \\
\hline Colorado & - & - & 52 & - & 52 \\
\hline Railroad & - & - & 52 & - & 52 \\
\hline 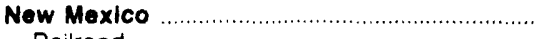 & - & - & 61 & - & 61 \\
\hline Railroad & - & - & 61 & - & 61 \\
\hline 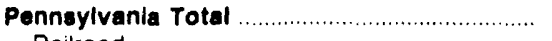 & - & - & • & - & * \\
\hline Railroad & - & - & 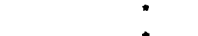 & - & - \\
\hline 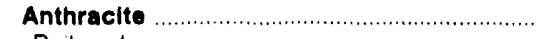 & - & - & 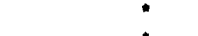 & - & * \\
\hline Railroad & - & - & $\bullet$ & - & $\cdot$ \\
\hline Utah & - & - & 1,209 & 43 & 1,252 \\
\hline 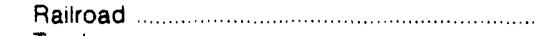 & - & - & 1,209 & 43 & 1,252 \\
\hline Truck & - & - & * & $\cdot$ & 1 \\
\hline 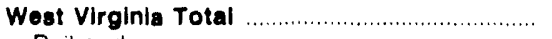 & - & - & " & - & : \\
\hline Railroad & - & - & * & - & $\cdot$ \\
\hline Northern & - & - & * & - & * \\
\hline 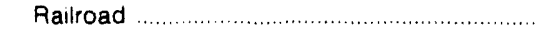 & - & - & • & - & * \\
\hline
\end{tabular}

See footnotes at end of table. 
Table 34. Domestic Distribution of U.S. Coal by Destination State, Consumer, Origin and Method of Transportation, January-June 1991 (Continued) (Thousand Short Tons)

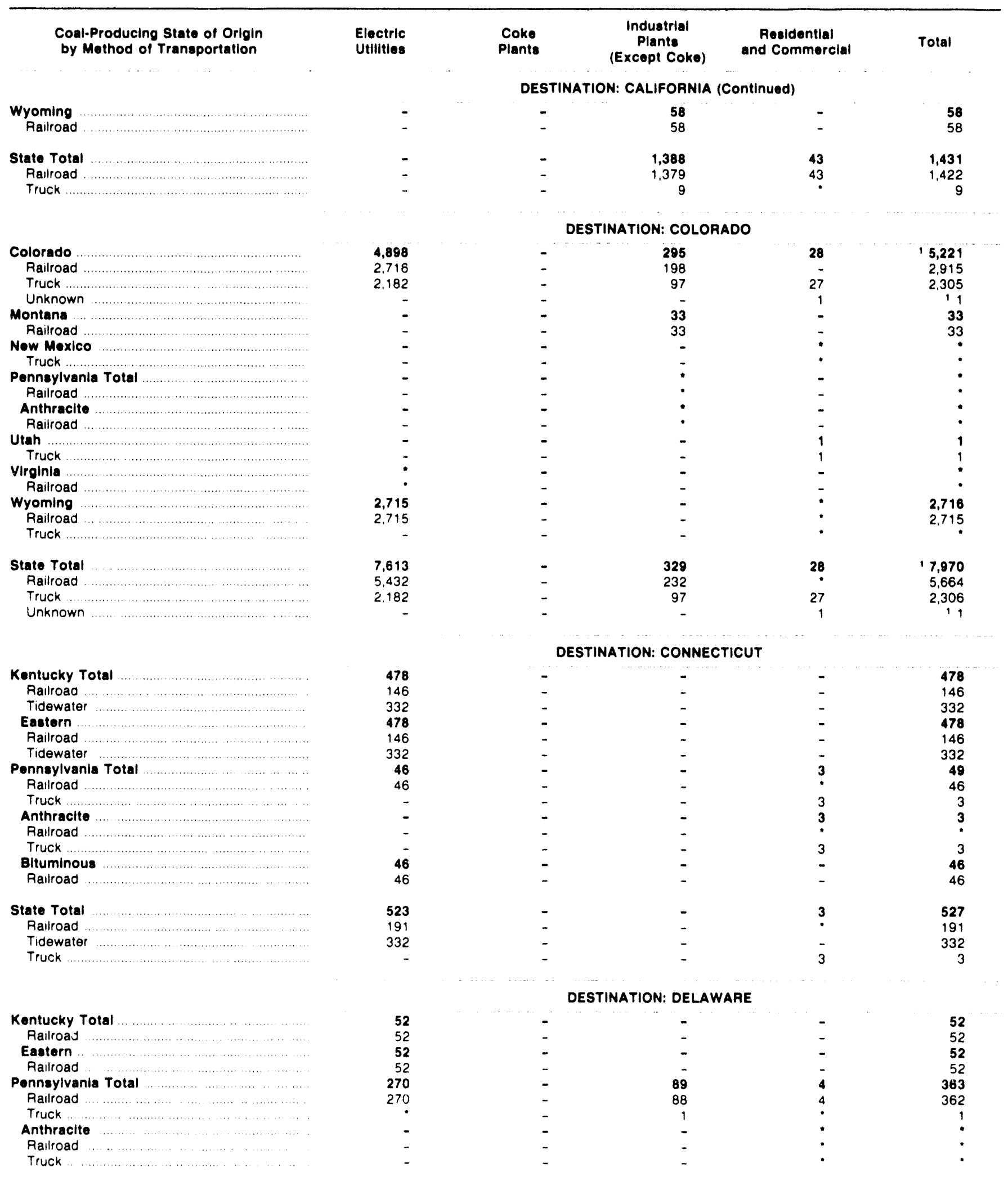

See footnotes at end of table. 
Table 34. Domestic Distribution of U.S. Coal by Destination State, Consumer, Origin and Method of Transportation, January-June 1991 (Continued) (Thousand Short Tons)

\begin{tabular}{|c|c|c|c|c|c|}
\hline $\begin{array}{l}\text { Coal-Producing State of Origin } \\
\text { by Method of Transportation }\end{array}$ & $\begin{array}{l}\text { Electric } \\
\text { Utilitles }\end{array}$ & $\begin{array}{l}\text { Coke } \\
\text { Plants }\end{array}$ & $\begin{array}{c}\text { Industrial } \\
\text { Plants } \\
\text { (Except Coke) }\end{array}$ & $\begin{array}{l}\text { Residential } \\
\text { and Commerclal }\end{array}$ & Total \\
\hline & \multicolumn{5}{|c|}{ DESTINATION: DELAWARE (Continued) } \\
\hline Bituminous & 270 & - & 89 & 4 & 363 \\
\hline$\ldots \ldots \ldots \ldots \ldots \ldots \ldots \ldots \ldots \ldots \ldots$ & 270 & - & 88 & 4 & 362 \\
\hline 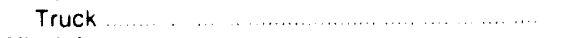 & $\cdot$ & - & 1 & - & 1 \\
\hline Virginia & 84 & - & - & - & 84 \\
\hline Railroad .......... & 84 & - & - & - & 84 \\
\hline West Virginia Total & 604 & - & 2 & - & 605 \\
\hline Railroad ................. & 604 & - & - & - & 604 \\
\hline Truck $\ldots \ldots \ldots \ldots$ & - & - & 2 & - & 2 \\
\hline Northern $\ldots \ldots \ldots \ldots$ & 238 & - & 2 & - & 240 \\
\hline 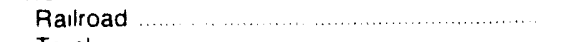 & 238 & - & - & - & 238 \\
\hline 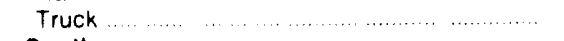 & - & - & 2 & - & 2 \\
\hline 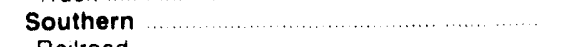 & 365 & - & - & - & 365 \\
\hline 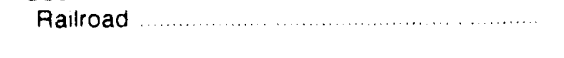 & 365 & - & - & - & 365 \\
\hline State Total. & 1,010 & - & 91 & 4 & 1,105 \\
\hline$\ldots \ldots \ldots \ldots$ & 1.010 & - & 88 & 4 & 1,102 \\
\hline \multirow{2}{*}{ Truck } & $\cdot$ & - & 3 & $\cdot$ & 3 \\
\hline & \multicolumn{5}{|c|}{ DESTINATION: DISTRICT OF COLUMBIA } \\
\hline Kentucky Total. & - & - & - & 33 & 33 \\
\hline Ralroad $\ldots \ldots \ldots \ldots \ldots \ldots$ & - & $\rightarrow$ & - & 33 & 33 \\
\hline 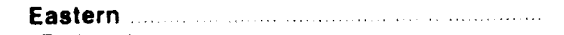 & - & - & - & 33 & 33 \\
\hline 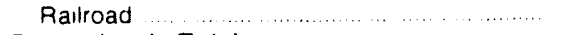 & - & - & - & 33 & 33 \\
\hline Pennsylvania Total $\ldots \ldots \ldots$ & - & - & - & $\bullet$ & 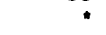 \\
\hline 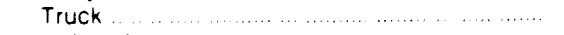 & - & - & - & $\cdot$ & - \\
\hline 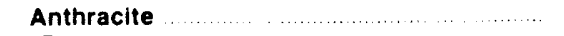 & - & - & - & - & * \\
\hline 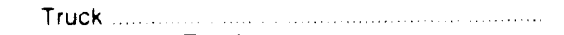 & - & - & - & $\cdot$ & - \\
\hline West Virginia Total $\ldots \ldots \ldots \ldots \ldots$ & - & - & - & 9 & 9 \\
\hline 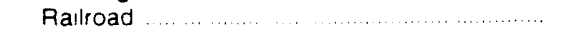 & - & - & - & 3 & 3 \\
\hline Truck $\ldots \ldots \ldots$ & - & - & - & 6 & 6 \\
\hline Northern $\ldots \ldots \ldots$ & - & - & - & 6 & 6 \\
\hline Truck $\ldots \ldots \ldots \ldots \ldots \ldots \ldots \ldots$ & - & - & - & 6 & 6 \\
\hline Southern & - & - & - & 3 & 3 \\
\hline 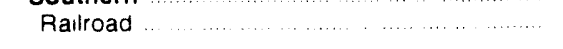 & - & - & - & 3 & 3 \\
\hline 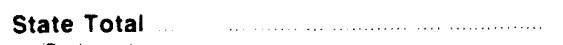 & - & - & - & 41 & 41 \\
\hline 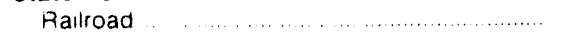 & - & - & - & 35 & 35 \\
\hline \multirow{2}{*}{ 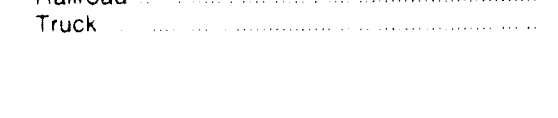 } & - & - & - & 6 & 6 \\
\hline & \multicolumn{5}{|c|}{ DESTINATION: FLORIDA } \\
\hline Alabama $\ldots \ldots \ldots$ & - & - & 36 & - & 36 \\
\hline 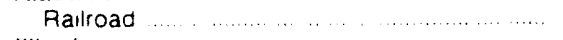 & - & - & 36 & - & 36 \\
\hline 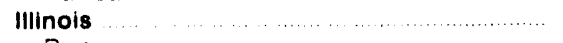 & 2,261 & - & - & - & 2,261 \\
\hline 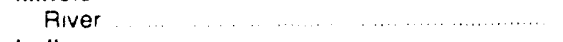 & 2,261 & - & - & - & 2,261 \\
\hline Indiana $\ldots \ldots \ldots \ldots \ldots \ldots$ & 111 & - & - & - & 111 \\
\hline 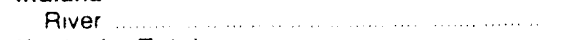 & 111 & - & - & - & 111 \\
\hline Kentucky Total $\ldots \ldots$ & 7,194 & - & 204 & - & 7,397 \\
\hline Railroad & 4,566 & - & 204 & - & 4,770 \\
\hline 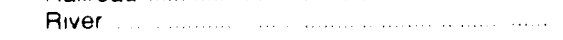 & 2.081 & - & - & - & 2,081 \\
\hline 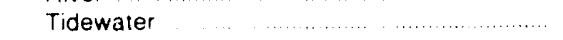 & 546 & - & - & - & 546 \\
\hline$\ldots \ldots \ldots \ldots \ldots \ldots \ldots \ldots \ldots \ldots \ldots \ldots \ldots \ldots$ & 5,333 & - & 204 & - & 5,537 \\
\hline$\ldots \ldots$ & 4,203 & - & 204 & - & 4,406 \\
\hline$\ldots \ldots \ldots$ & 731 & - & - & - & 731 \\
\hline Tidewater & 399 & - & - & - & 399 \\
\hline Western & 1,861 & - & - & - & 1,861 \\
\hline Rasiroad $\ldots \ldots \ldots \ldots \ldots \ldots$ & 363 & - & - & - & 363 \\
\hline River $\ldots \ldots \ldots \ldots$ & 1.350 & - & - & - & 1.350 \\
\hline 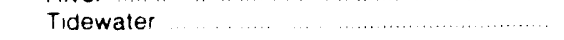 & 147 & - & - & - & 147 \\
\hline 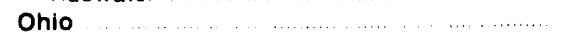 & 167 & - & - & - & 167 \\
\hline Tidewater & 167 & - & - & - & 167 \\
\hline Pennsylvania Total & 66 & - & 3 & * & 70 \\
\hline Ralload $\ldots$ & - & - & * & - & $\cdot$ \\
\hline Tidewater $\ldots \ldots \ldots \ldots$ & 66 & - & - & - & 66 \\
\hline Truck & $\cdot$ & - & 3 & • & 3 \\
\hline
\end{tabular}

See footnotes at end of lable 
Table 34. Domestic Distribution of U.S. Coal by Destination State, Consumer, Origin and Method of Transportation, January-June 1991 (Continued) (Thousand Short Tons)

\begin{tabular}{|c|c|c|c|c|c|}
\hline $\begin{array}{l}\text { Coal-Producing State of Origin } \\
\text { by Method of Transportation }\end{array}$ & $\begin{array}{l}\text { Electric } \\
\text { Utilities }\end{array}$ & $\begin{array}{l}\text { Coke } \\
\text { Plants }\end{array}$ & $\begin{array}{c}\text { Industrial } \\
\text { Plants } \\
\text { (Except Coke) }\end{array}$ & $\begin{array}{l}\text { Residential } \\
\text { and Commercial }\end{array}$ & Total \\
\hline & \multicolumn{5}{|c|}{ DESTINATION: FLORIDA (Continued) } \\
\hline Anthracite & 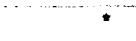 & - & 3 & • & 3 \\
\hline 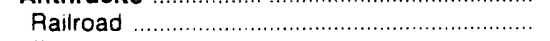 & - & - & $\cdot$ & - & - \\
\hline 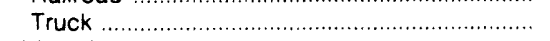 & $\cdot$ & - & 3 & $\cdot$ & 3 \\
\hline 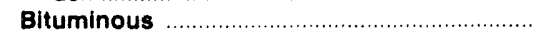 & 66 & - & - & - & 66 \\
\hline 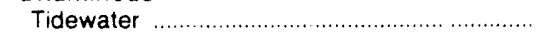 & 66 & - & - & - & 66 \\
\hline 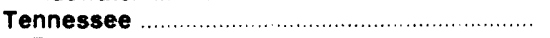 & 82 & - & 49 & - & 131 \\
\hline Railroad & - & - & 48 & - & 48 \\
\hline 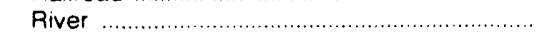 & 82 & - & - & - & 82 \\
\hline 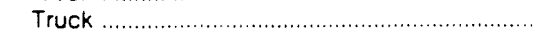 & - & - & $\dot{\bullet}$ & - & $\cdot$ \\
\hline 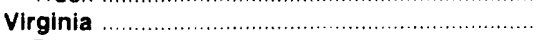 & 158 & - & 76 & - & 234 \\
\hline 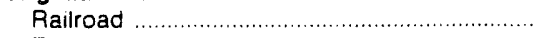 & 158 & - & 76 & - & 234 \\
\hline 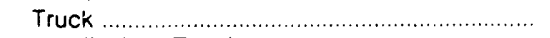 & - & - & • & - & $\dot{-}$ \\
\hline West Virginia Total & 978 & - & - & - & 978 \\
\hline 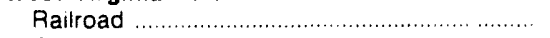 & 402 & - & - & - & 402 \\
\hline River & 2 & - & - & - & 2 \\
\hline 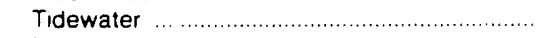 & 574 & - & - & - & 574 \\
\hline 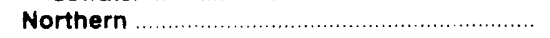 & 217 & - & - & - & 217 \\
\hline 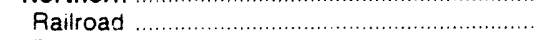 & 7 & - & - & - & 7 \\
\hline 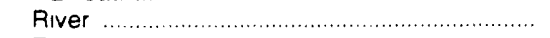 & $\cdot$ & - & - & - & $\cdot$ \\
\hline Tidewater & 209 & - & - & - & 209 \\
\hline 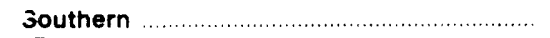 & 761 & - & - & - & 764 \\
\hline Railroad & 394 & - & - & - & 394 \\
\hline 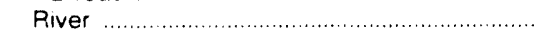 & 2 & - & - & - & 2 \\
\hline Tidewater & 365 & - & - & - & 365 \\
\hline State Total & 11,017 & - & 367 & * & 11,384 \\
\hline Railroad & 5.126 & - & 364 & - & 5,489 \\
\hline 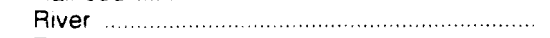 & 4,537 & - & - & - & 4,537 \\
\hline Tidewater & 1,354 & - & - & - & 1,354 \\
\hline \multirow[t]{2}{*}{ Truck ......... } & 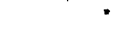 & - & 4 & $\cdot$ & 4 \\
\hline & \multicolumn{5}{|c|}{ DESTINATION: GEORGIA } \\
\hline Alabama. & 39 & - & 2 & - & 41 \\
\hline 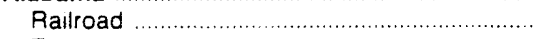 & 39 & - & - & - & 39 \\
\hline 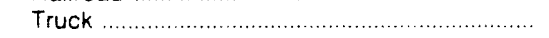 & - & - & 2 & - & 2 \\
\hline 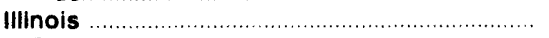 & 1,829 & - & - & - & 1,829 \\
\hline 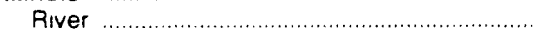 & 1,829 & - & - & - & 1,829 \\
\hline 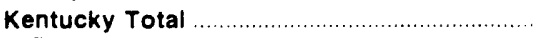 & 6,096 & - & 641 & 4 & 6,741 \\
\hline 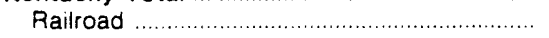 & 6,096 & - & 608 & 4 & 6,708 \\
\hline 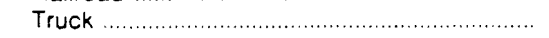 & 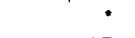 & - & 3i. & $\cdot$ & 33 \\
\hline 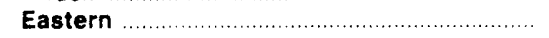 & 5,427 & - & 634 & 4 & 6,065 \\
\hline 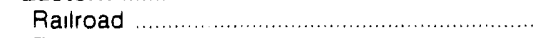 & 5.427 & - & 601 & 4 & 6,032 \\
\hline Truck & . & - & 33 & * & 33 \\
\hline Western & 668 & - & 8 & - & 676 \\
\hline Railroad & 669 & - & 8 & - & 676 \\
\hline 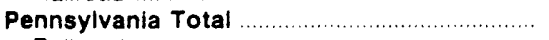 & - & - & • & * & 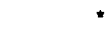 \\
\hline 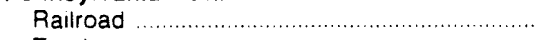 & - & - & $\cdot$ & - & - \\
\hline 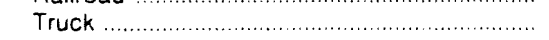 & - & - & $\cdot$ & $\cdot$ & - \\
\hline Anthracite & - & - & * & $\cdot$ & - \\
\hline Railroad & - & - & * & - & • \\
\hline 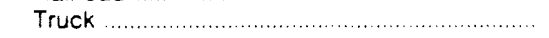 & - & - & $\cdot$ & * & $\cdot$ \\
\hline Tennessee & 32 & - & 184 & - & 216 \\
\hline Railroad & 32 & - & 133 & - & 165 \\
\hline 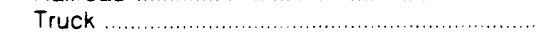 & - & - & 51 & - & 51 \\
\hline Virginia & 1,443 & - & 187 & 1 & 1,631 \\
\hline 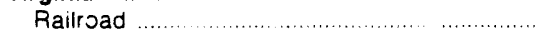 & 1.443 & - & 187 & 1 & 1.631 \\
\hline West Virginia Total & 1,062 & - & - & - & 1,062 \\
\hline 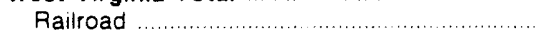 & 1.062 & - & - & - & 1.062 \\
\hline Southern & 1,062 & - & - & - & 1,062 \\
\hline Railroad & 1.062 & - & - & - & 1.062 \\
\hline Wyoming & 1,127 & - & - & - & 1,127 \\
\hline Railroad & 1.127 & - & - & - & 1.127 \\
\hline State Total & 11,629 & - & 1,014 & 4 & 12,647 \\
\hline Railroad & 9.799 & - & 928 & 4 & 10.732 \\
\hline River & 1.829 & - & - & - & 1.829 \\
\hline Truck & $\cdot$ & - & $\underline{\operatorname{AE}}$ & $\cdot$ & RE \\
\hline
\end{tabular}

Se€ footnotes at end of table 
Table 34. Domestic Distribution of U.S. Coal by Destination State, Consumer, Origin and Method of Transportation, January-Jue 1991 (Continued) (Thousand Short Tons)

\begin{tabular}{|c|c|c|c|c|c|}
\hline $\begin{array}{l}\text { Coal-Producing State of Origin } \\
\text { by Method of Transportation }\end{array}$ & $\begin{array}{l}\text { Electric } \\
\text { Utilities }\end{array}$ & $\begin{array}{l}\text { Coke } \\
\text { Plants }\end{array}$ & $\begin{array}{c}\text { Industrial } \\
\text { Plants } \\
\text { (Except Coke) }\end{array}$ & $\begin{array}{l}\text { Resldential } \\
\text { and Commercial }\end{array}$ & Total \\
\hline (n.................. & \multicolumn{5}{|c|}{ DESTINATION: IDAHO } \\
\hline Colorado & - & - & 11 & - & 11 \\
\hline 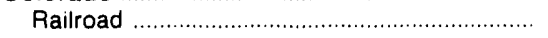 & - & - & 11 & - & 11 \\
\hline Utah & - & - & 20 & 32 & 52 \\
\hline 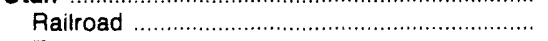 & - & - & - & 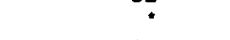 & 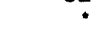 \\
\hline Truck & - & - & 20 & 32 & 51 \\
\hline 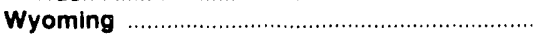 & - & - & 213 & 3 & 216 \\
\hline 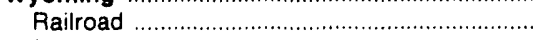 & - & - & 133 & . & 133 \\
\hline 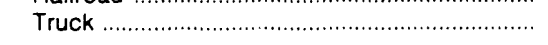 & - & - & 79 & 3 & 82 \\
\hline State Total & - & - & 243 & 36 & 279 \\
\hline Railroad & - & - & 145 & 1 & 145 \\
\hline \multirow[t]{2}{*}{ 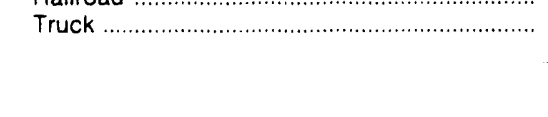 } & - & - & 99 & 35 & 134 \\
\hline & \multicolumn{5}{|c|}{ DESTINATION: ILLINOIS } \\
\hline Colorado & 315 & - & - & - & 315 \\
\hline 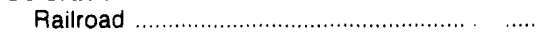 & 315 & - & - & - & 315 \\
\hline Ililnois & 7,879 & - & 1,278 & 85 & 9,242 \\
\hline 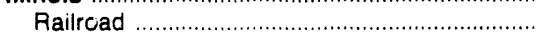 & 4,453 & - & 384 & 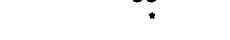 & 4,837 \\
\hline River & 727 & - & 25 & - & 753 \\
\hline 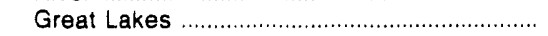 & 10 & - & - & - & 10 \\
\hline Truck & 1,453 & - & 857 & 83 & 2,392 \\
\hline Tramway, Conveyor, and Slurry Pipeline ........ & 1,237 & - & - & - & 1,237 \\
\hline 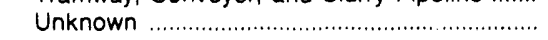 & - & - & 12 & 2 & 14 \\
\hline 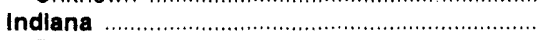 & 738 & - & 322 & 16 & 1,076 \\
\hline Railroad & 137 & - & - & - & 137 \\
\hline River & 321 & - & 194 & - & 515 \\
\hline 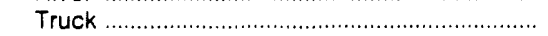 & 281 & - & 128 & 16 & 424 \\
\hline Kentucky Total & 814 & 134 & 202 & - & 1,151 \\
\hline 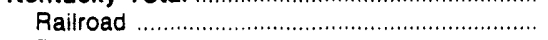 & 342 & 7 & 44 & - & 394 \\
\hline River & 456 & 127 & 144 & - & 726 \\
\hline Truck & 16 & - & 15 & - & 31 \\
\hline Eastern & 672 & 134 & 77 & - & 883 \\
\hline 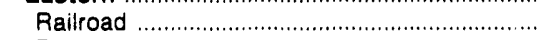 & 342 & 7 & 44 & - & 394 \\
\hline 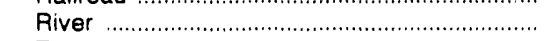 & 330 & 127 & 22 & - & 478 \\
\hline 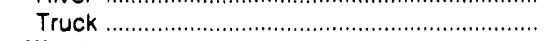 & - & - & 12 & - & 12 \\
\hline Western & 142 & - & 125 & - & 267 \\
\hline 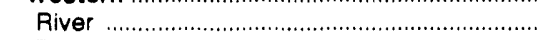 & 126 & - & 122 & - & 248 \\
\hline Truck & 16 & - & 3 & - & 19 \\
\hline 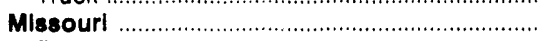 & - & - & * & - & 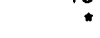 \\
\hline Truck & - & - & • & - & - \\
\hline Montana & 1,757 & - & - & - & 1,757 \\
\hline 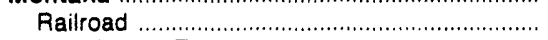 & 1,757 & - & - & - & 1,757 \\
\hline 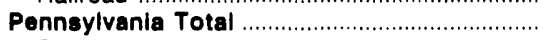 & - & - & 21 & * & 21 \\
\hline Railroad & - & - & 4 & $\cdot$ & 5 \\
\hline River & - & - & 16 & - & 16 \\
\hline Truck & - & - & 1 & $\cdot$ & 1 \\
\hline Anthracite & - & - & 5 & * & 5 \\
\hline 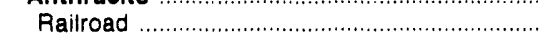 & - & - & 4 & * & 5 \\
\hline Truck & - & - & 1 & n & 1 \\
\hline gltuminous & - & - & 16 & - & 16 \\
\hline River & - & - & 16 & - & 16 \\
\hline 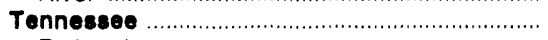 & 10 & - & 18 & - & 29 \\
\hline Railroad & 10 & - & - & - & 10 \\
\hline 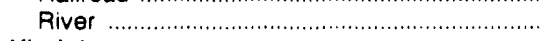 & - & - & 18 & - & 18 \\
\hline 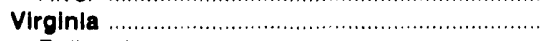 & - & 312 & - & - & 312 \\
\hline 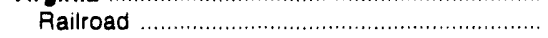 & - & 138 & - & - & 138 \\
\hline 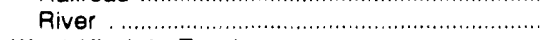 & - & 174 & - & - & 174 \\
\hline 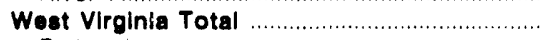 & 383 & 319 & 55 & * & 757 \\
\hline Railroad & 150 & 178 & 18 & - & 346 \\
\hline 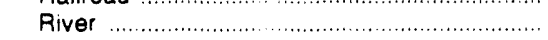 & 233 & 141 & 33 & - & 407 \\
\hline 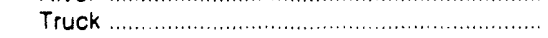 & - & - & 5 & • & 5 \\
\hline 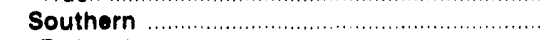 & 383 & 319 & 55 & • & 757 \\
\hline Railroad & 150 & 178 & 18 & - & 346 \\
\hline 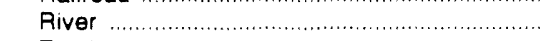 & 233 & 141 & 33 & - & 407 \\
\hline Truck & - & - & 5 & • & 5 \\
\hline
\end{tabular}

Sae fontngtes at and of tabla 
Table 34. Domestic Distribution of U.S. Coal by Destination State, Consumer, Origin and Method of Transportation, January-June 1991 (Continued) (Thousand Short Tons)

\begin{tabular}{|c|c|c|c|c|c|}
\hline $\begin{array}{l}\text { Coal-Producing State of Origin } \\
\text { by Method of Transportation }\end{array}$ & $\begin{array}{l}\text { Electric } \\
\text { Utilities }\end{array}$ & $\begin{array}{l}\text { Coke } \\
\text { Plants }\end{array}$ & $\begin{array}{c}\text { Industrial } \\
\text { Plants } \\
\text { (Except Coke) }\end{array}$ & $\begin{array}{l}\text { Realdential } \\
\text { and Commerelal }\end{array}$ & Total \\
\hline & \multicolumn{5}{|c|}{ DESTINATION: ILLINOIS (Continued) } \\
\hline Wyoming. & 2,064 & - & 352 & - & 2,416 \\
\hline 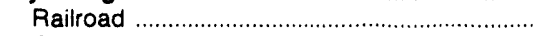 & 1,864 & - & 352 & - & 2,216 \\
\hline Great Lakes .................................................... & 201 & - & - & - & 201 \\
\hline S:aid Total & 13,961 & 766 & 2,249 & 102 & 17,077 \\
\hline 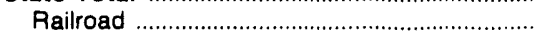 & 9,027 & 324 & 802 & ? & 10.153 \\
\hline 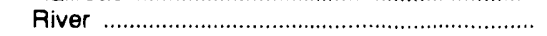 & 1,737 & 442 & 430 & - & 2,609 \\
\hline 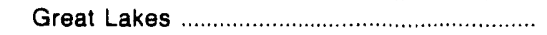 & 211 & - & - & - & 211 \\
\hline 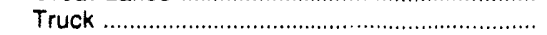 & 1,749 & - & 1,005 & 99 & 2,853 \\
\hline Tramway, Conveyor, and Slurry Pipeline ........ & 1,237 & - & - & - & 1,237 \\
\hline \multirow[t]{2}{*}{ 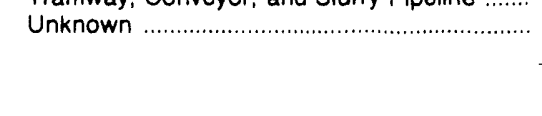 } & - & - & 12 & 2 & 14 \\
\hline & \multicolumn{5}{|c|}{ DESTINATION: INDIANA } \\
\hline Alabama . & 6 & - & - & - & 6 \\
\hline 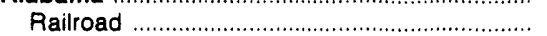 & 6 & - & - & - & 6 \\
\hline 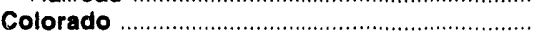 & 429 & - & - & - & 429 \\
\hline 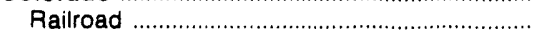 & 429 & - & - & - & 429 \\
\hline 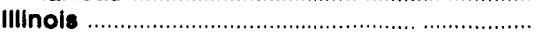 & 4,774 & 363 & 91 & * & 5,228 \\
\hline 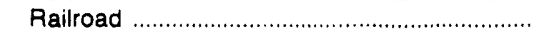 & 4,292 & 363 & 41 & - & 4,696 \\
\hline 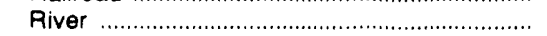 & 156 & - & - & - & 156 \\
\hline 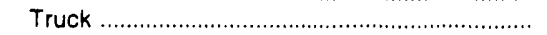 & 326 & - & 50 & . & 376 \\
\hline 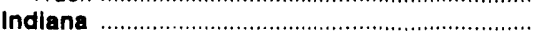 & 8,547 & - & 1,325 & 221 & 10,092 \\
\hline 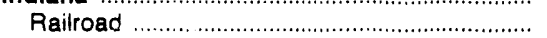 & 5,914 & - & 788 & - & 6,702 \\
\hline 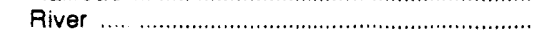 & 639 & - & 8 & - & 647 \\
\hline 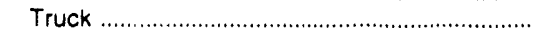 & 1,641 & - & 519 & 220 & 2,380 \\
\hline Tramway, Conveyor, and Slurry Pipeline ........ & 352 & - & - & - & 352 \\
\hline Unknown & - & - & 11 & 1 & 11 \\
\hline 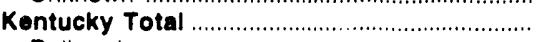 & 1,989 & 808 & 10 & ! & 2,808 \\
\hline Railroad …… & 280 & 747 & - & - & 1,027 \\
\hline 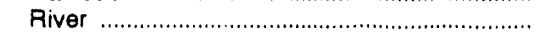 & 1,707 & 61 & - & - & 1,768 \\
\hline 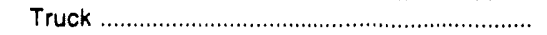 & 2 & 1 & 10 & • & 13 \\
\hline 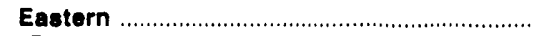 & 282 & 808 & 10 & • & 1,101 \\
\hline 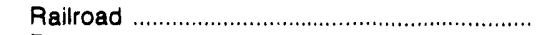 & 280 & 747 & - & - & 1,027 \\
\hline 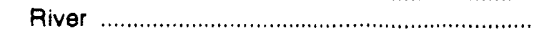 & - & 61 & - & - & 61 \\
\hline 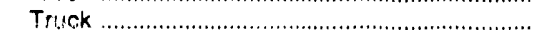 & 2 & 1 & 10 & • & 13 \\
\hline 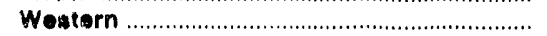 & 9,707 & - & - & - & 1,707 \\
\hline 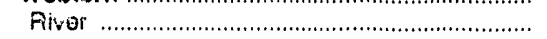 & 1,707 & - & - & - & 1,707 \\
\hline 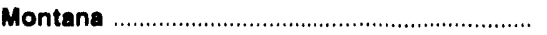 & 305 & - & - & - & 305 \\
\hline 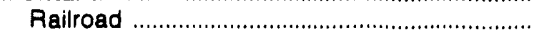 & 305 & - & - & - & 305 \\
\hline Ohlo & 20 & - & 52 & 24 & 96 \\
\hline 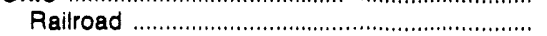 & - & - & - & 11 & 11 \\
\hline 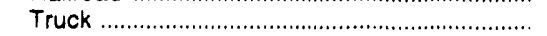 & 20 & - & 52 & 12 & 84 \\
\hline 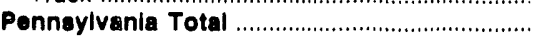 & - & 161 & 11 & 1 & 173 \\
\hline 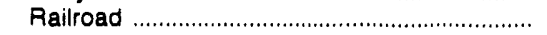 & - & 161 & $\because$ & - & 161 \\
\hline 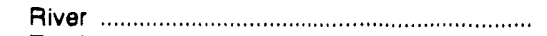 & - & - & 11 & - & 11 \\
\hline 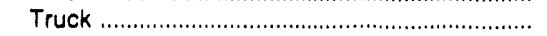 & - & - & ! & 1 & 1 \\
\hline Anthracite & - & - & • & 1 & 1 \\
\hline 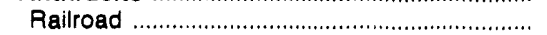 & - & - & • & - & : \\
\hline 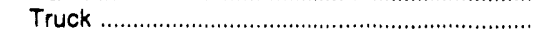 & - & - & . & 1 & 1 \\
\hline 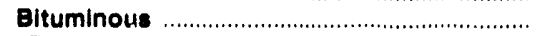 & - & 161 & 11 & - & 173 \\
\hline 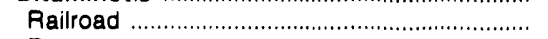 & - & 161 & - & - & 161 \\
\hline 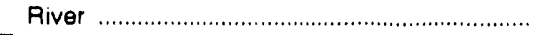 & - & - & 11 & - & 11 \\
\hline 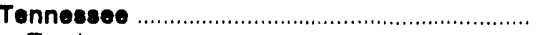 & - & - & 1 & - & 1 \\
\hline Truck ………… & - & - & 1 & - & 1 \\
\hline 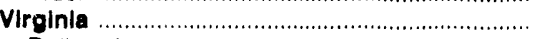 & - & 602 & - & - & 602 \\
\hline 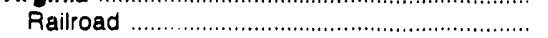 & - & 556 & - & - & 556 \\
\hline 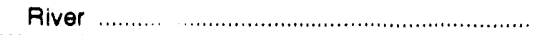 & - & 46 & - & - & 46 \\
\hline Weat Virginia Total & 13 & 1,156 & 549 & - & 1,837 \\
\hline 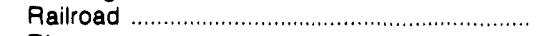 & 120 & 1,156 & 381 & - & 1,665 \\
\hline River & - & - & 139 & - & 139 \\
\hline 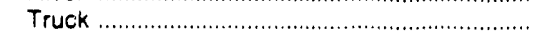 & 2 & - & 29 & - & 32 \\
\hline 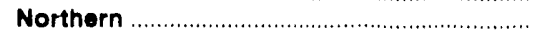 & $=$ & - & 181 & - & 161 \\
\hline 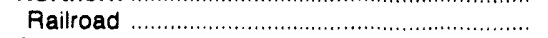 & - & - & 161 & - & 161 \\
\hline Southern & 131 & 1,156 & 388 & - & 1,675 \\
\hline 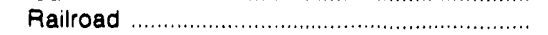 & 129 & 1,156 & 219 & - & 1,504 \\
\hline 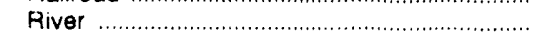 & - & - & 139 & - & 139 \\
\hline 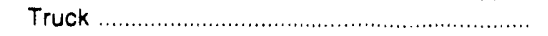 & 2 & - & 29 & - & 32 \\
\hline
\end{tabular}

See footnotes at end of table. 
Table 34. Domestic Distribution of U.S. Coal by Destination State, Consumer, Origin and Methoc of Transportation, January-June 1991 (Continued) (Thousand Short Tons)

\begin{tabular}{|c|c|c|c|c|c|}
\hline $\begin{array}{l}\text { Coal-Producing State of Origin } \\
\text { by Method of Transportation }\end{array}$ & $\begin{array}{l}\text { Electric } \\
\text { Utilities }\end{array}$ & $\begin{array}{l}\text { Coke } \\
\text { Plants }\end{array}$ & $\begin{array}{c}\text { Industrial } \\
\text { Plants } \\
\text { (Except Coke) }\end{array}$ & $\begin{array}{l}\text { Residential } \\
\text { and Commercial }\end{array}$ & Total \\
\hline & \multicolumn{5}{|c|}{ DESTINATION: INDIANA (Continued) } \\
\hline Wyoming & 5,400 & - & - & - & 5,400 \\
\hline Railroad & 1,219 & - & - & - & 1,219 \\
\hline River & 4,181 & - & - & - & 4,181 \\
\hline State Total & 21,602 & 3,090 & 2,040 & 245 & 26,976 \\
\hline 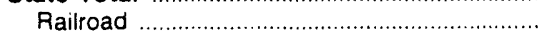 & 12,574 & 2,983 & 1,210 & 11 & 16,778 \\
\hline River & 6,683 & 106 & 158 & - & 6,947 \\
\hline 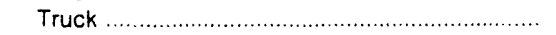 & 1,992 & 1 & 661 & 233 & 2,887 \\
\hline Tramway, Conveyor, and Slurry Pipeline ....... & 352 & - & - & - & 352 \\
\hline \multirow[t]{2}{*}{ 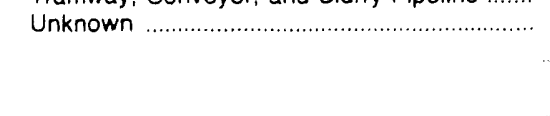 } & - & - & 11 & 1 & 11 \\
\hline & \multicolumn{5}{|c|}{ DESTINATION: IOWA } \\
\hline Illinols & 725 & - & 23 & 13 & 761 \\
\hline Railroad …… & 156 & - & - & 13 & 169 \\
\hline 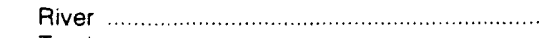 & 559 & - & 23 & - & 583 \\
\hline 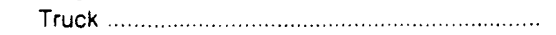 & 10 & - & - & - & 10 \\
\hline 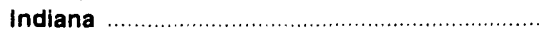 & 517 & - & 116 & - & 634 \\
\hline Railroad & 264 & - & 24 & - & 287 \\
\hline River & 254 & - & 93 & - & 346 \\
\hline lowa & $17 \underline{2}$ & - & - & 1 & 173 \\
\hline 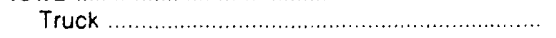 & 172 & - & - & 1 & 173 \\
\hline Kentucky Total & 36 & - & 139 & 138 & 313 \\
\hline Railroad & - & - & 1 & - & 1 \\
\hline River & 36 & - & 138 & 138 & 312 \\
\hline Truck & - & - & • & - & \\
\hline 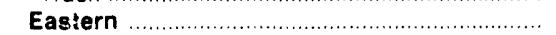 & - & - & 55 & 97 & 151 \\
\hline Railroad & - & - & 1 & - & 1 \\
\hline 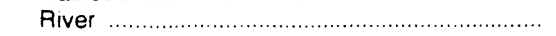 & - & - & 54 & 97 & 150 \\
\hline Truck & - & - & • & - & • \\
\hline Western & 36 & - & 84 & 41 & 162 \\
\hline River & 36 & - & 84 & 41 & 162 \\
\hline 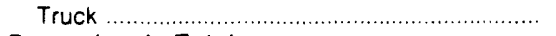 & - & - & • & - & " \\
\hline Pennsylvania Total & - & - & 83 & • & 83 \\
\hline Railroad & - & - & 83 & - & 83 \\
\hline 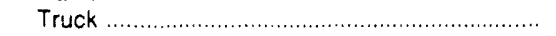 & - & - & • & - & " \\
\hline Anthracite & - & - & 17 & * & 17 \\
\hline Railroad & - & - & 16 & • & 17 \\
\hline 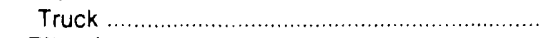 & - & - & • & - & • \\
\hline Bituminous & - & - & 67 & - & 67 \\
\hline 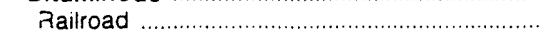 & - & - & 67 & - & 67 \\
\hline Utah & - & - & - & * & * \\
\hline 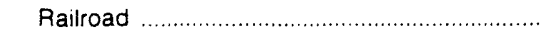 & - & - & - & : & - \\
\hline 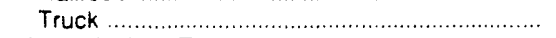 & - & - & - & - & - \\
\hline West Virginia Total & - & - & 20 & - & 20 \\
\hline 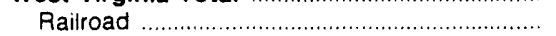 & - & - & 8 & - & 8 \\
\hline River & - & - & 12 & - & 12 \\
\hline 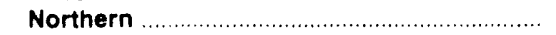 & - & - & 1 & - & 1 \\
\hline 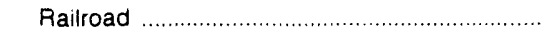 & - & - & 1 & - & 1 \\
\hline Southern & - & - & 19 & - & 19 \\
\hline 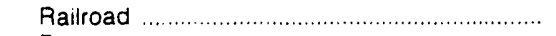 & - & - & 7 & - & 7 \\
\hline River & - & - & 12 & - & 12 \\
\hline Wyoming & 6,935 & - & 78 & - & 7,013 \\
\hline Railroad & 6,654 & - & 78 & - & 6.732 \\
\hline 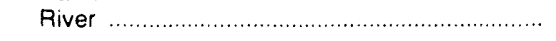 & 281 & - & - & - & 281 \\
\hline 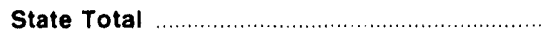 & 8,386 & - & 459 & 152 & 8,996 \\
\hline Railroad & 7,074 & - & 193 & 13 & 7,280 \\
\hline 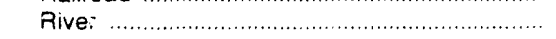 & 1,130 & - & 266 & 138 & 1,534 \\
\hline \multirow[t]{2}{*}{ 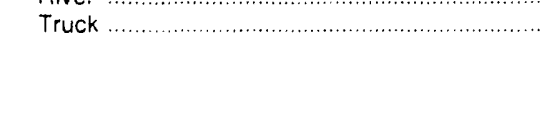 } & 182 & - & . & 1 & 183 \\
\hline & \multicolumn{5}{|c|}{ DESTINATION: KANSAS } \\
\hline 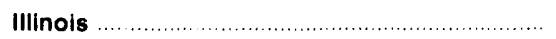 & 388 & - & - & - & 388 \\
\hline 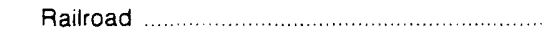 & 388 & - & - & - & 388 \\
\hline Indiana & - & - & 6 & - & 6 \\
\hline Truck & - & - & 6 & - & 6 \\
\hline
\end{tabular}


Table 34. Domestic Distribution of U.S. Coal by Destination State, Consumer, Origin and Method of Transportation, January-June 1991 (Continued) (Thousand Short Tons)

\begin{tabular}{|c|c|c|c|c|c|}
\hline $\begin{array}{l}\text { Coal-Producing State of Origin } \\
\text { by Method of Transportation }\end{array}$ & $\begin{array}{l}\text { Electric } \\
\text { Utilities }\end{array}$ & $\begin{array}{l}\text { Coke } \\
\text { Plants }\end{array}$ & $\begin{array}{c}\text { Industrial } \\
\text { Plants } \\
\text { (Except Coke) }\end{array}$ & $\begin{array}{l}\text { Residential } \\
\text { and Commercial }\end{array}$ & Total \\
\hline & \multicolumn{5}{|c|}{ DESTINATION: KANSAS (Continued) } \\
\hline Kansas … . & 56 & - & 16 & - & 72 \\
\hline 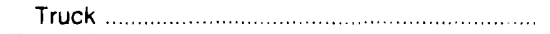 & 56 & - & 16 & * & 72 \\
\hline 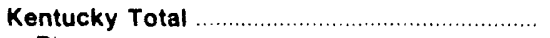 & - & - & 3 & - & 3 \\
\hline 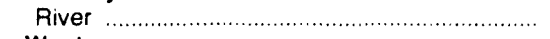 & - & - & 3 & - & 3 \\
\hline 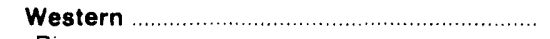 & - & - & 3 & - & 3 \\
\hline 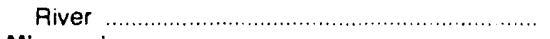 & - & - & 3 & - & 3 \\
\hline Missouri & - & - & 3 & - & 3 \\
\hline 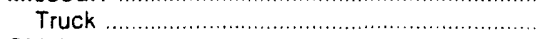 & - & - & 3 & - & 3 \\
\hline 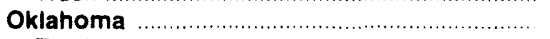 & 2 & - & 7 & - & 9 \\
\hline Truck & 2 & - & 7 & - & 9 \\
\hline Pennsylvania Total & - & - & * & - & * \\
\hline Railroad & - & - & $\cdot$ & - & - \\
\hline Truck & - & - & $\cdot$ & - & - \\
\hline Anthracite & - & - & * & - & - \\
\hline 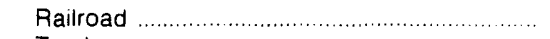 & - & - & $\cdot$ & - & * \\
\hline 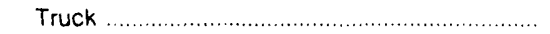 & - & - & • & - & - \\
\hline 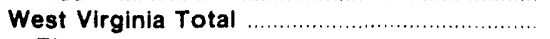 & - & - & 5 & - & 5 \\
\hline 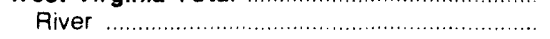 & - & .. & 5 & - & 5 \\
\hline Southern & - & - & 5 & - & 5 \\
\hline 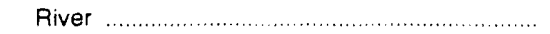 & - & - & 5 & - & 5 \\
\hline 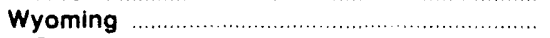 & 6,461 & - & - & - & 6,461 \\
\hline Railroad & 6,461 & - & - & - & 6.461 \\
\hline State Total & 6,907 & - & 41 & * & 6,949 \\
\hline Railroad & 6,849 & - & $\because$ & - & 6.8 .19 \\
\hline River & - & - & 8 & - & 8 \\
\hline \multirow[t]{2}{*}{ ( } & 58 & - & 33 & $\cdot$ & 91 \\
\hline & \multicolumn{5}{|c|}{ DESTINATION: KENTUCKY } \\
\hline ................ & 1,094 & - & 18 & - & 1,112 \\
\hline (n) & 113 & - & - & - & 113 \\
\hline 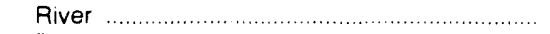 & 920 & - & - & - & 920 \\
\hline Truck & 61 & - & 18 & - & 79 \\
\hline Kentucky Total & 10,596 & 50 & 595 & 101 & 111,343 \\
\hline Railroad & 3,791 & 46 & 247 & 18 & 4,102 \\
\hline River & 2,553 & - & 2 & $\cdot$ & 2.555 \\
\hline Truck & 2,977 & 4 & 339 & 46 & 3.365 \\
\hline Tramway, Conveyor, and Siurry Pipeline ....... & 1,275 & - & - & - & 1,275 \\
\hline 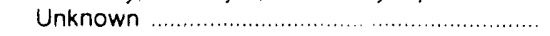 & - & - & 8 & 37 & 46 \\
\hline 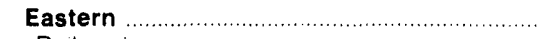 & 3,003 & 50 & 574 & 90 & 13,717 \\
\hline 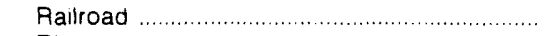 & 818 & 46 & 247 & 18 & 1.129 \\
\hline 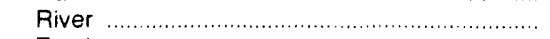 & 757 & - & 2 & - & 759 \\
\hline 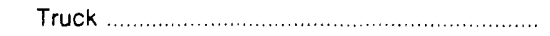 & 1.427 & 4 & 318 & 36 & 1,784 \\
\hline 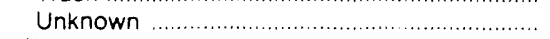 & - & - & 8 & 36 & 145 \\
\hline 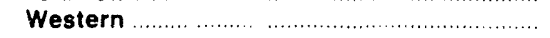 & 7,593 & - & 21 & 11 & 7,626 \\
\hline Railroad & 2.972 & - & - & 1 & 2,973 \\
\hline River & 1,796 & - & - & $\cdot$ & 1.797 \\
\hline 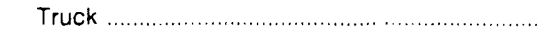 & 1,550 & - & 21 & 10 & 1.581 \\
\hline Tramway, Conveyor, and Slurry Pipeline ........ & 1,275 & - & - & - & 1,275 \\
\hline 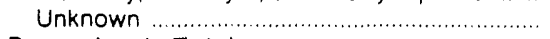 & - & - & - & 1 & 1 \\
\hline Pennsylvania Total & - & - & 11 & $\star$ & 11 \\
\hline Railroad & - & - & 11 & - & 11 \\
\hline 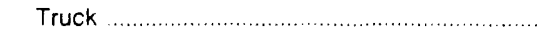 & - & - & - & $\cdot$ & $\cdot$ \\
\hline 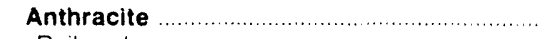 & - & - & 11 & * & 11 \\
\hline 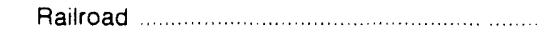 & - & - & 11 & - & 11 \\
\hline 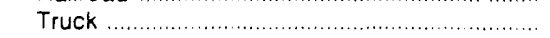 & - & - & - & $\cdot$ & $\cdot$ \\
\hline 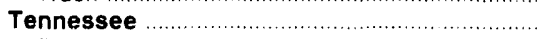 & 306 & - & 1 & 1 & 308 \\
\hline 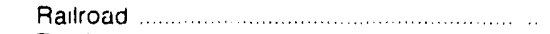 & 305 & - & - & - & 305 \\
\hline 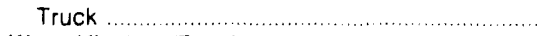 & 1 & - & 1 & 1 & 3 \\
\hline West Virginia Totai & 1,532 & 355 & 306 & - & 2,192 \\
\hline 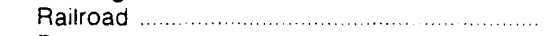 & 595 & 307 & 109 & - & 1.010 \\
\hline River & 784 & 48 & 40 & - & 872 \\
\hline 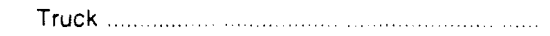 & 153 & - & 157 & - & 309 \\
\hline (1, & 9 & - & - & - & 9 \\
\hline 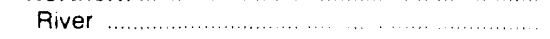 & 9 & - & - & - & 9 \\
\hline
\end{tabular}

See footnotes at end of table 
Table 34. Domestic Distribution of U.S. Coal by Destination State, Consumer, Origin and Method of Transportation, January-June 1991 (Continued) (Thousand Short Tons)

\begin{tabular}{|c|c|c|c|c|c|}
\hline $\begin{array}{l}\text { Coal-Producing State of Origin } \\
\text { by Method of Trafingortation }\end{array}$ & $\begin{array}{l}\text { Electric } \\
\text { Utilities }\end{array}$ & $\begin{array}{l}\text { Coke } \\
\text { Plants }\end{array}$ & $\begin{array}{c}\text { Industrlal } \\
\text { Plants } \\
\text { (Except Coke) }\end{array}$ & $\begin{array}{c}\text { Residential } \\
\text { and Commercial }\end{array}$ & Total \\
\hline & \multicolumn{5}{|c|}{ DESTINATION: KENTUCKY (Continued) } \\
\hline Southern & 1,523 & 355 & 306 & - & 2,183 \\
\hline 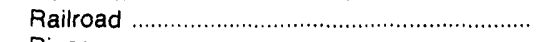 & 595 & 307 & 109 & - & 1,010 \\
\hline 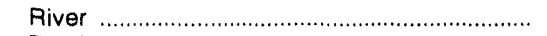 & 775 & 48 & 40 & - & 863 \\
\hline 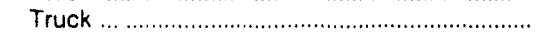 & 153 & - & 157 & - & 309 \\
\hline 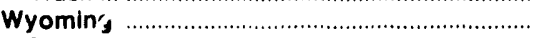 & 183 & - & - & - & 183 \\
\hline 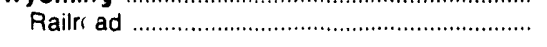 & 183 & - & - & - & 183 \\
\hline State Total & 13,711 & 405 & 931 & 102 & $1,15,148$ \\
\hline 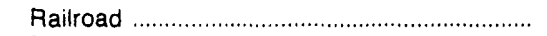 & 4,986 & 353 & 366 & 18 & 5,724 \\
\hline 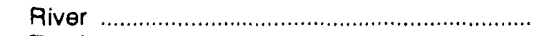 & 4,257 & 48 & 42 & • & 4,348 \\
\hline 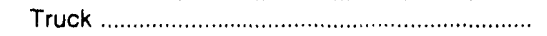 & 3,192 & 4 & 514 & 47 & 3,757 \\
\hline Tramway, Conveyor, and Slurry Pipeline ....... & 1,275 & - & - & - & 1,275 \\
\hline \multirow[t]{2}{*}{ 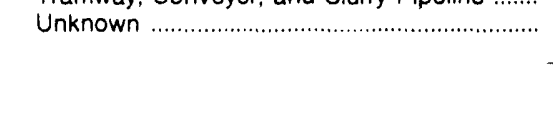 } & - & - & 8 & 37 & 146 \\
\hline & \multicolumn{5}{|c|}{ DESTINATION: LOUISIANA } \\
\hline 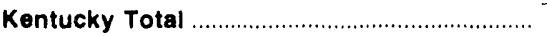 & 319 & - & 50 & - & 369 \\
\hline 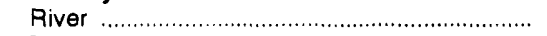 & 315 & - & 50 & - & 365 \\
\hline 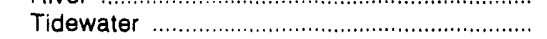 & 4 & - & - & - & 4 \\
\hline Eastern & 102 & - & 50 & - & 152 \\
\hline 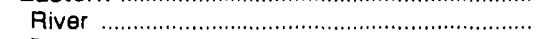 & 97 & - & 50 & - & 147 \\
\hline 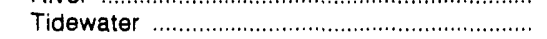 & 4 & - & - & - & 4 \\
\hline 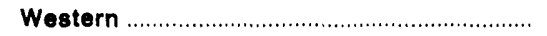 & 217 & - & - & - & 217 \\
\hline 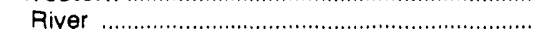 & 217 & - & - & - & 217 \\
\hline Loulslana .................................................... & 1,287 & - & - & - & 1,287 \\
\hline Truck & 177 & - & - & - & 177 \\
\hline Tramway, Conveyor, and Slurry Pipeline ........ & 1,111 & - & - & - & 1,111 \\
\hline 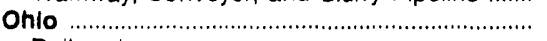 & 1 & - & • & - & 1 \\
\hline 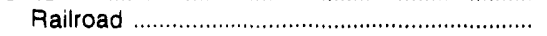 & - & - & • & - & : \\
\hline River & 1 & - & - & - & 1 \\
\hline 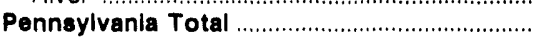 & - & - & • & - & • \\
\hline Railroad ...1. & - & - & • & - & * \\
\hline 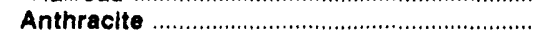 & - & - & • & - & * \\
\hline 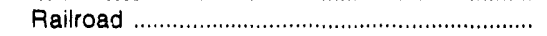 & - & - & • & - & * \\
\hline 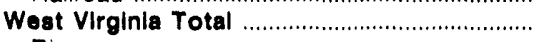 & 2 & - & 12 & - & 14 \\
\hline 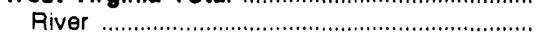 & 2 & - & 12 & - & 14 \\
\hline Southern & 2 & - & 12 & - & 14 \\
\hline River & 2 & - & 12 & - & 14 \\
\hline 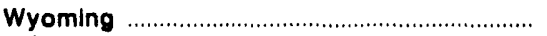 & 4,183 & - & 194 & - & 4,377 \\
\hline 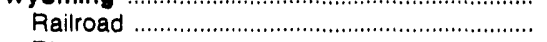 & 1,849 & - & 194 & - & 2,043 \\
\hline 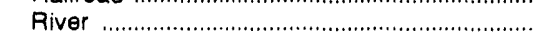 & 2,334 & - & - & - & 2,334 \\
\hline State Total & 5,792 & - & 256 & - & 6,048 \\
\hline 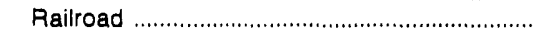 & 1,849 & - & 194 & - & 2,043 \\
\hline 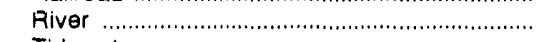 & 2,652 & - & 62 & - & 2,714 \\
\hline 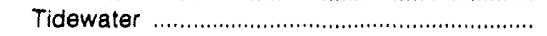 & 4 & - & - & - & 4 \\
\hline 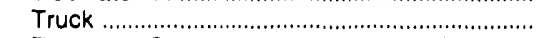 & 177 & - & - & - & 177 \\
\hline \multirow[t]{2}{*}{ Tramway, Conveyor, and Slurry Pipeline ........ } & 1,111 & - & - & - & 1,111 \\
\hline & \multicolumn{5}{|c|}{ DESTINATION: MAINE } \\
\hline Pennsylvanla Total & - & - & - & 3 & 3 \\
\hline 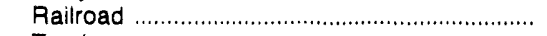 & - & - & - & 1 & 1 \\
\hline 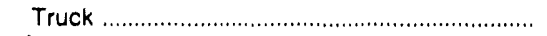 & - & - & - & 2 & 2 \\
\hline Anthracite & - & - & - & 3 & 3 \\
\hline 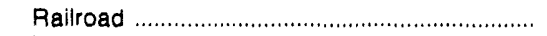 & - & - & - & 1 & 1 \\
\hline 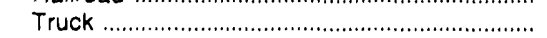 & - & - & - & 2 & 2 \\
\hline 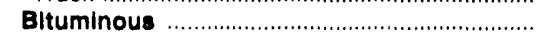 & - & - & - & * & . \\
\hline 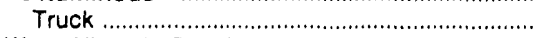 & - & - & - & - & * \\
\hline 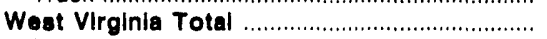 & - & - & 10 & - & 10 \\
\hline 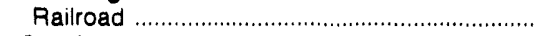 & - & - & 10 & - & 10 \\
\hline Southern & - & - & 10 & - & 10 \\
\hline 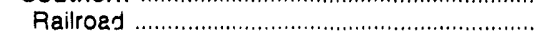 & - & - & 10 & - & 10 \\
\hline State Total & - & - & 10 & 3 & 12 \\
\hline 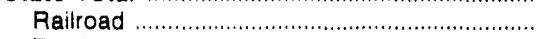 & - & - & 10 & 1 & 11 \\
\hline Truck & - & - & - & 2 & 2 \\
\hline
\end{tabular}

See footnotes at end of table. 
Table 34. Domestic Distribution of U.S. Coal by Destination State, Consumer, Origin and Method of Transportation, January-June 1991 (Continued) (Thousand Short Tons)

\begin{tabular}{|c|c|c|c|c|c|}
\hline $\begin{array}{l}\text { Coal-Producing State of Origin } \\
\text { by Method of Transportation }\end{array}$ & $\begin{array}{l}\text { Eloctric } \\
\text { Utillties }\end{array}$ & $\begin{array}{l}\text { Coke } \\
\text { Plants }\end{array}$ & $\begin{array}{c}\text { Industrial } \\
\text { Plants } \\
\text { (Except Coke) }\end{array}$ & $\begin{array}{l}\text { Residential } \\
\text { and Commerclal }\end{array}$ & Total \\
\hline & \multicolumn{5}{|c|}{ DESTINATION: MARYLAND } \\
\hline 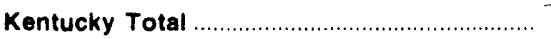 & 194 & 78 & 87 & - & 359 \\
\hline 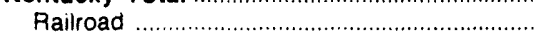 & 51 & - & 87 & - & 138 \\
\hline 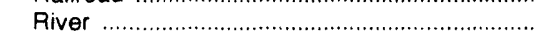 & - & 78 & - & - & 78 \\
\hline 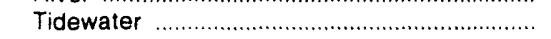 & 143 & - & - & - & 143 \\
\hline Eastern & 194 & 78 & 87 & - & 359 \\
\hline 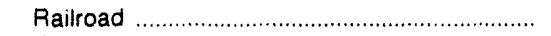 & 51 & - & 87 & - & 138 \\
\hline 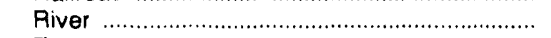 & - & 78 & - & - & 78 \\
\hline 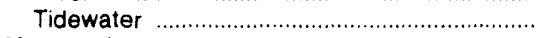 & 143 & - & - & - & 143 \\
\hline Maryland & 426 & - & 184 & 1 & 611 \\
\hline 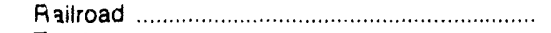 & 401 & - & 125 & - & 526 \\
\hline 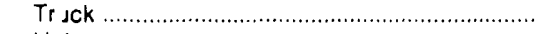 & 25 & - & 48 & 1 & 74 \\
\hline 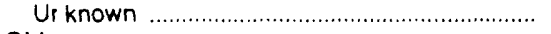 & - & - & 12 & $\bullet$ & 12 \\
\hline Ohio & 7 & - & - & - & 7 \\
\hline 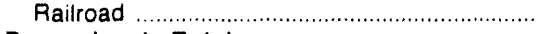 & 7 & - & - & - & 7 \\
\hline 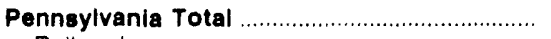 & 1,139 & 56 & 78 & 2 & 1,275 \\
\hline 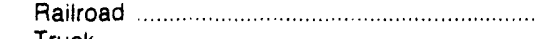 & 1,072 & 56 & 30 & " & 1,158 \\
\hline 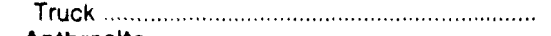 & 67 & - & 48 & 2 & 116 \\
\hline 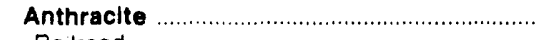 & - & - & 3 & 2 & 4 \\
\hline 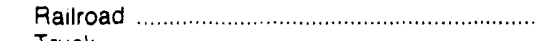 & - & - & 3 & 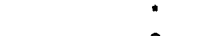 & 3 \\
\hline 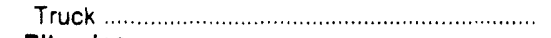 & - & - & $\cdot$ & 2 & 2 \\
\hline 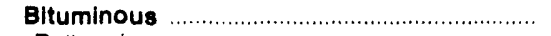 & 1,139 & 56 & 75 & - & 1,270 \\
\hline 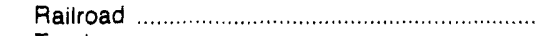 & 1,072 & 56 & 27 & - & 1,156 \\
\hline 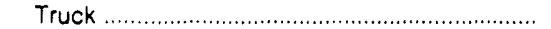 & 67 & - & 48 & - & 115 \\
\hline 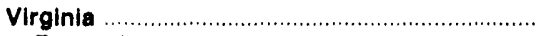 & $\cdot$ & 79 & - & • & 79 \\
\hline Railroad & - & 42 & - & - & 42 \\
\hline 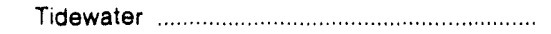 & • & 36 & - & - & 36 \\
\hline Truck & - & - & - & " & * \\
\hline 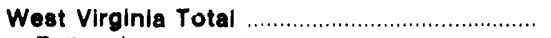 & 2,763 & 54 & 265 & 10 & 3,091 \\
\hline Railroad & 2,371 & 54 & 226 & - & 2,650 \\
\hline 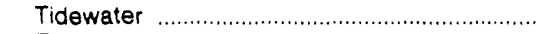 & 388 & - & - & - & 388 \\
\hline 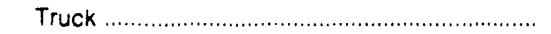 & 5 & - & 40 & 10 & 54 \\
\hline 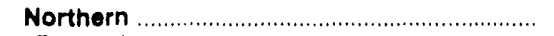 & 2,030 & - & 119 & 10 & 2,159 \\
\hline 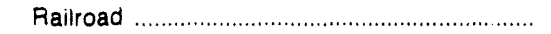 & 1,824 & - & 80 & - & 1,904 \\
\hline 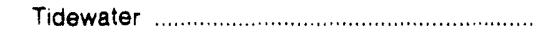 & 206 & - & - & - & 206 \\
\hline 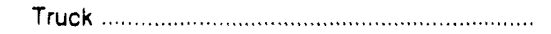 & - & - & 40 & 10 & 49 \\
\hline Southern & 733 & 54 & 146 & - & 933 \\
\hline Railroad . . & 546 & 54 & 146 & - & 746 \\
\hline 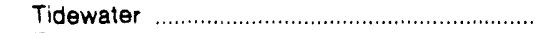 & 182 & - & - & - & 182 \\
\hline 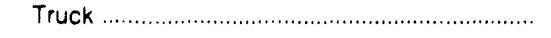 & 5 & - & - & - & 5 \\
\hline State Total & 4,529 & 267 & 614 & 13 & 5,422 \\
\hline 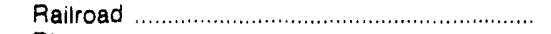 & 3,902 & 152 & 467 & - & 4,522 \\
\hline 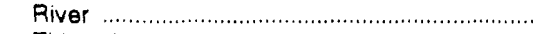 & - & 78 & - & - & 78 \\
\hline 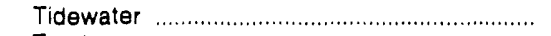 & 530 & 36 & - & - & 567 \\
\hline 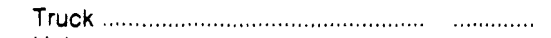 & 96 & - & 135 & 12 & 244 \\
\hline \multirow{2}{*}{ 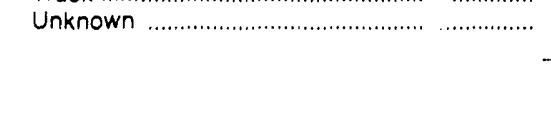 } & - & - & 12 & $\cdot$ & 12 \\
\hline & \multicolumn{5}{|c|}{ DESTINATION: MASSACHUSETTS } \\
\hline 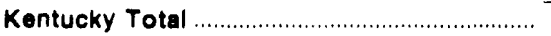 & 232 & - & 7 & $\cdot$ & 239 \\
\hline 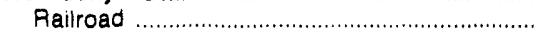 & 15 & - & 4 & - & 20 \\
\hline 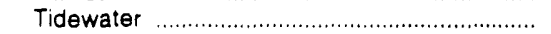 & 217 & - & - & - & 217 \\
\hline 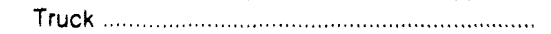 & - & - & 2 & - & 2 \\
\hline Eastern & 232 & - & 7 & • & 239 \\
\hline 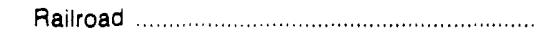 & 15 & - & 4 & $\cdot$ & 20 \\
\hline 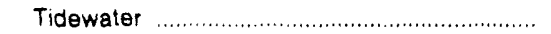 & 217 & - & - & - & 217 \\
\hline 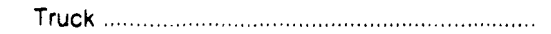 & - & - & 2 & - & 2 \\
\hline Ohlo & - & - & 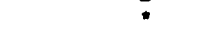 & • & 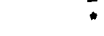 \\
\hline 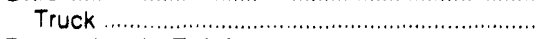 & - & - & $\cdot$ & * & $\cdot$ \\
\hline 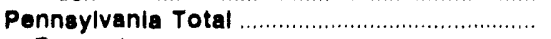 & 69 & - & 2 & 8 & 79 \\
\hline 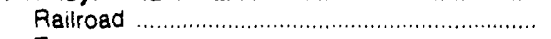 & 68 & - & $\overrightarrow{1}$ & 2 & 70 \\
\hline 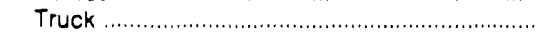 & 1 & - & 2 & 6 & 9 \\
\hline 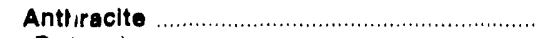 & - & - & $\cdot$ & 8 & 8 \\
\hline 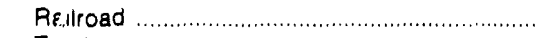 & - & - & $\cdot$ & 2 & 2 \\
\hline 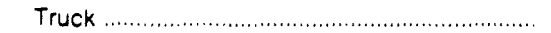 & - & - & - & 6 & 6 \\
\hline Eltuminous & 69 & - & 2 & - & 71 \\
\hline 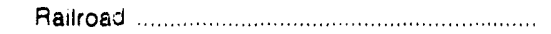 & 68 & - & 1 & - & 68 \\
\hline 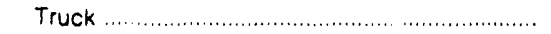 & 1 & - & 2 & - & $?$ \\
\hline
\end{tabular}

See footnotes at end of table. 
Table 34. Domestic Distribution of U.S. Coal by Destination State, Consumer, Origin and Method of Transportation, January-June 1991 (Continued) (Thousand Short Tons)

\begin{tabular}{|c|c|c|c|c|c|}
\hline $\begin{array}{l}\text { Coal-Producing State of Origin } \\
\text { by Method of Transportation }\end{array}$ & $\begin{array}{l}\text { Electric } \\
\text { Utilities }\end{array}$ & $\begin{array}{l}\text { Coke } \\
\text { Plants }\end{array}$ & $\begin{array}{c}\text { Industrial } \\
\text { Plants } \\
\text { (Except Coke) }\end{array}$ & $\begin{array}{l}\text { Residential } \\
\text { and Commercial }\end{array}$ & Total \\
\hline & \multicolumn{5}{|c|}{ DESTINATION: MASSACHUSETTS (Continued) } \\
\hline Virginia & 316 & - & - & - & 316 \\
\hline 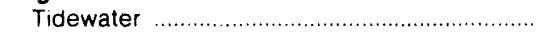 & 316 & - & - & - & 316 \\
\hline West Virginia Total & 920 & - & 17 & - & 938 \\
\hline Railroad & 337 & - & 17 & - & 354 \\
\hline 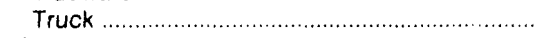 & 20 & - & 1 & - & 21 \\
\hline Northern & 835 & - & 1 & - & 836 \\
\hline 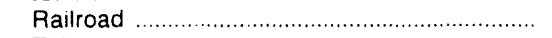 & 304 & - & 1 & - & 305 \\
\hline 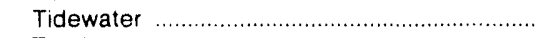 & 528 & - & - & - & 528 \\
\hline Truck & 3 & - & - & - & 3 \\
\hline 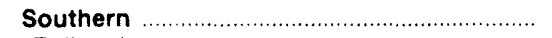 & 85 & - & 16 & - & 101 \\
\hline 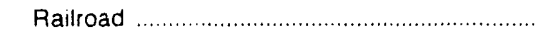 & 33 & - & 16 & - & 48 \\
\hline 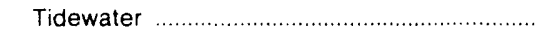 & 36 & - & - & - & 36 \\
\hline 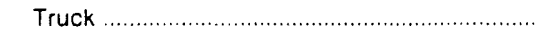 & 17 & - & 1 & - & 17 \\
\hline State Total & 1,537 & - & 27 & 8 & 1,572 \\
\hline Railroad & 420 & - & 22 & 2 & 444 \\
\hline 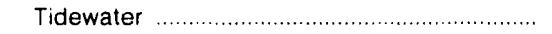 & 1,096 & - & - & - & 1,096 \\
\hline \multirow[t]{2}{*}{ 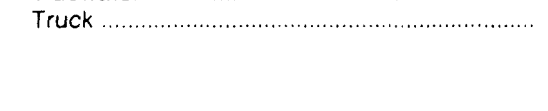 } & 21 & - & 5 & 6 & 32 \\
\hline & \multicolumn{5}{|c|}{ DESTINATION: MICHIGAN } \\
\hline Indiana & 48 & - & 93 & 3 & 144 \\
\hline 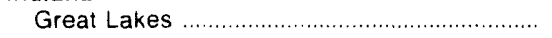 & 48 & - & 92 & - & 139 \\
\hline 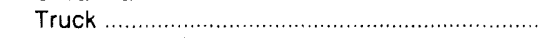 & - & - & 1 & 3 & 4 \\
\hline Kentucky Total ....... & 3,354 & 4 & 551 & 73 & 3,982 \\
\hline Railroad & 3,144 & - & 293 & 72 & 3,509 \\
\hline 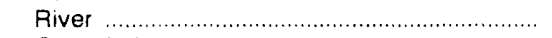 & - & - & 8 & - & 8 \\
\hline 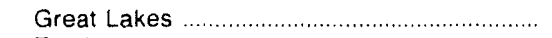 & 210 & - & 223 & 1 & 434 \\
\hline 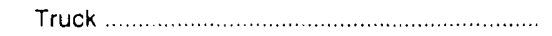 & - & 4 & 27 & $\cdot$ & 31 \\
\hline 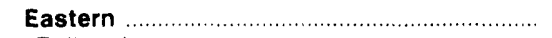 & 3,293 & 4 & 545 & 73 & 3,915 \\
\hline 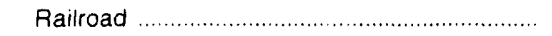 & 3,144 & - & 293 & 72 & 3,509 \\
\hline 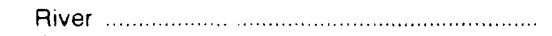 & - & - & 8 & - & 8 \\
\hline 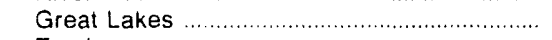 & 149 & - & 217 & 1 & 367 \\
\hline Montana & 5,118 & - & 18 & - & 5,135 \\
\hline 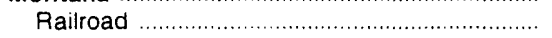 & 2,218 & - & - & - & 2,218 \\
\hline 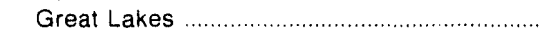 & 2,900 & - & 18 & - & 2,918 \\
\hline Ohio & 70 & - & 229 & - & 299 \\
\hline 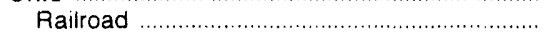 & 70 & - & - & - & 70 \\
\hline 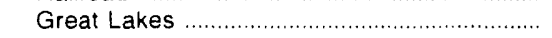 & - & - & 34 & - & 34 \\
\hline Truck … & $*$ & - & 195 & - & 195 \\
\hline 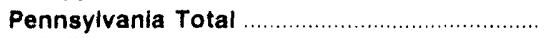 & 614 & 35 & 42 & 12 & 704 \\
\hline 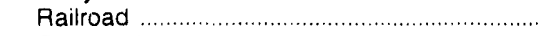 & 456 & 7 & 21 & - & 484 \\
\hline 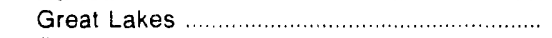 & 158 & 28 & 21 & - & 207 \\
\hline 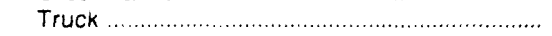 & - & - & 1 & 12 & 13 \\
\hline 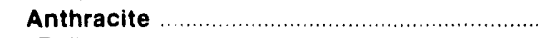 & - & - & 1 & * & 1 \\
\hline Railroad & - & - & " & - & * \\
\hline 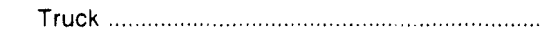 & - & - & 1 & * & 1 \\
\hline 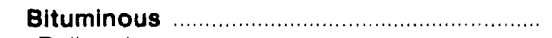 & 614 & 35 & 42 & 12 & 704 \\
\hline 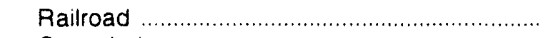 & 456 & 7 & 21 & - & 484 \\
\hline 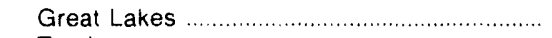 & 158 & 28 & 21 & - & 207 \\
\hline 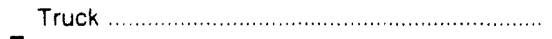 & - & - & - & 12 & 12 \\
\hline Tennessee & * & - & 18 & - & 18 \\
\hline Great Lakes & $\bullet$ & - & 18 & - & 18 \\
\hline Virginia & 2 & - & - & - & 2 \\
\hline 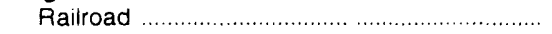 & 2 & - & - & - & 2 \\
\hline West Virginia Total & 2,997 & 181 & 457 & 3 & 3,637 \\
\hline 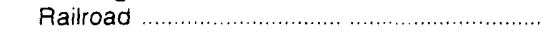 & 2,987 & 157 & 389 & 2 & 3,535 \\
\hline 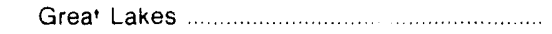 & 9 & 24 & 65 & 1 & 100 \\
\hline 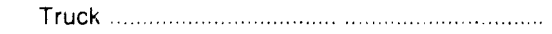 & - & - & 2 & * & 3 \\
\hline 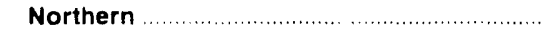 & 4 & - & 17 & - & 21 \\
\hline 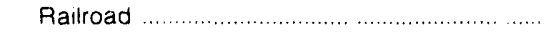 & 4 & - & - & - & 4 \\
\hline Great Lakes & - & - & 17 & - & 17 \\
\hline
\end{tabular}

See footnotes at end of table. 
Table 34. Domestic Distribution of U.S. Coal by Destination State, Consumer, Origin and Method of Transportation, January-June 1991 (Continued) (Thousand Short Tons)

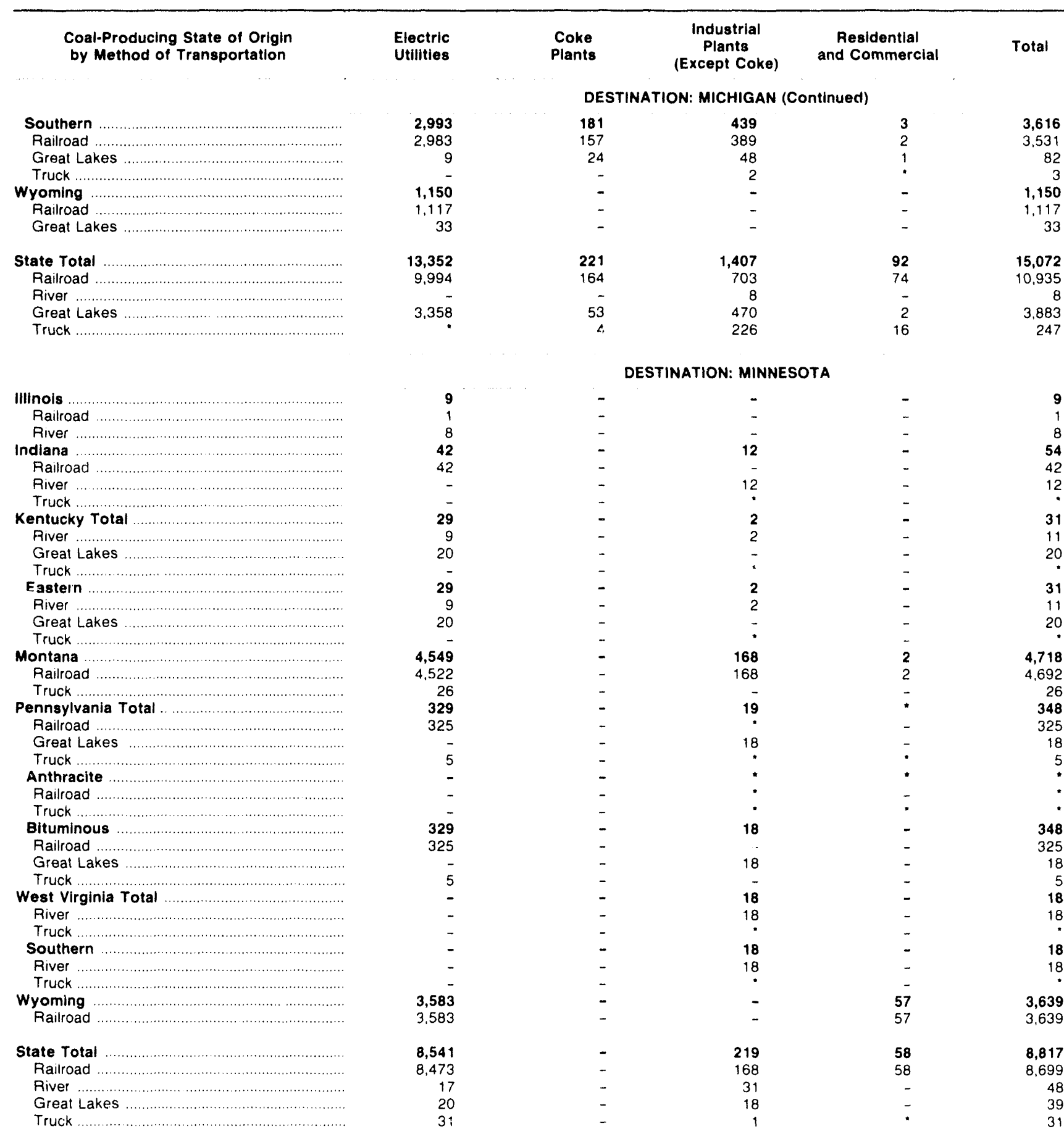

DESTINATION: MISSISSIPPI

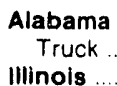

Illinois

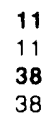

See footnotes at end of table 
Table 34. Domestic Distribution of U.S. Coal by Destination State, Consumer, Origin and Method of Transportation, January-June 1991 (Continued) (Thousand Short Tons)

\begin{tabular}{|c|c|c|c|c|c|}
\hline $\begin{array}{l}\text { Coal-Producing State of Origin } \\
\text { by Method of Transportation }\end{array}$ & $\begin{array}{l}\text { Eloctric } \\
\text { Utillities }\end{array}$ & $\begin{array}{l}\text { Coke } \\
\text { Plants }\end{array}$ & $\begin{array}{c}\text { Industrial } \\
\text { Planto } \\
\text { (Except Coke) }\end{array}$ & $\begin{array}{l}\text { Residential } \\
\text { and Commercial }\end{array}$ & Total \\
\hline & \multicolumn{5}{|c|}{ DESTINATION: MISSISSIPPI (Continued) } \\
\hline Kentucky Total. & 1,093 & - & 23 & - & 1,116 \\
\hline 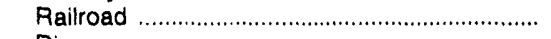 & 1,051 & - & - & - & 1,051 \\
\hline 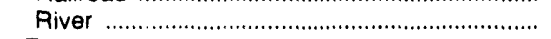 & 42 & - & 23 & - & 65 \\
\hline 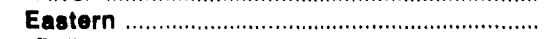 & 1,051 & - & - & - & 1,051 \\
\hline 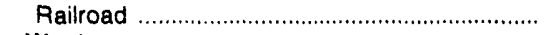 & 1.051 & - & - & - & 1,051 \\
\hline 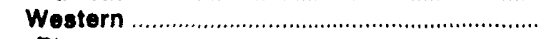 & 42 & - & 23 & - & 65 \\
\hline 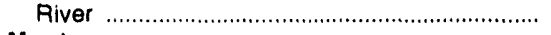 & 42 & - & 23 & - & 65 \\
\hline 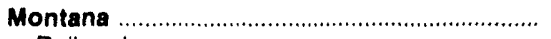 & 23 & - & - & - & 23 \\
\hline 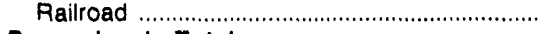 & 23 & - & - & - & 23 \\
\hline 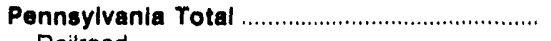 & - & - & 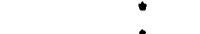 & - & " \\
\hline 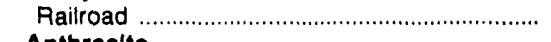 & - & - & 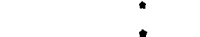 & - & " \\
\hline Anthracite & - & - & $:$ & - & : \\
\hline 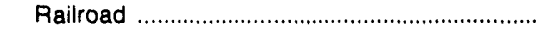 & - & - & 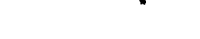 & - & " \\
\hline 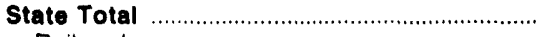 & 1,823 & - & 72 & - & 1,895 \\
\hline 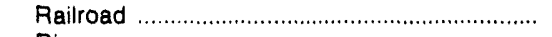 & 1.073 & - & $\cdot$ & - & 1,074 \\
\hline 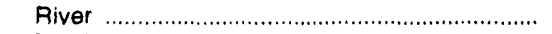 & 750 & - & 60 & - & 810 \\
\hline \multirow[t]{2}{*}{ 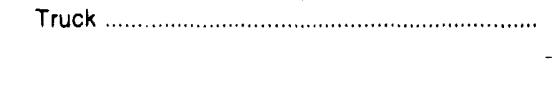 } & - & - & 11 & - & 11 \\
\hline & \multicolumn{5}{|c|}{ DESTINATION: MISSOUAI } \\
\hline 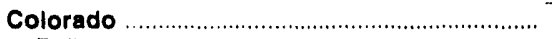 & 208 & - & 5 & - & 213 \\
\hline 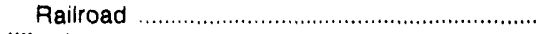 & 208 & - & 5 & - & 213 \\
\hline Illinols & 5,897 & - & 345 & 61 & 6,303 \\
\hline 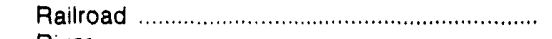 & 4,273 & - & - & - & 4,273 \\
\hline 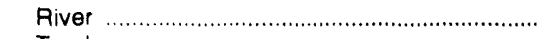 & 1,624 & - & 75 & - & 1,699 \\
\hline 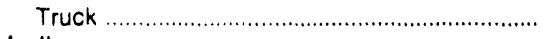 & - & - & 270 & 61 & 331 \\
\hline Indiana & 68 & - & 17 & 2 & 88 \\
\hline 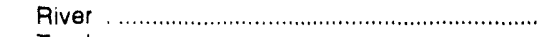 & 69 & - & 2 & - & 71 \\
\hline 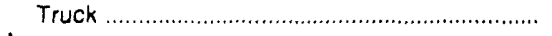 & - & - & 15 & 2 & 17 \\
\hline lowa & 2 & - & - & - & 2 \\
\hline 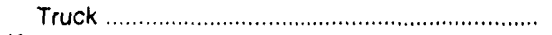 & 2 & - & . & - & 2 \\
\hline 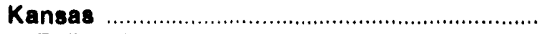 & 134 & - & 51 & 1 & 185 \\
\hline 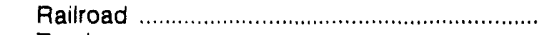 & 27 & - & - & - & 27 \\
\hline 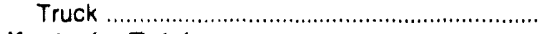 & 106 & - & 51 & 1 & 158 \\
\hline 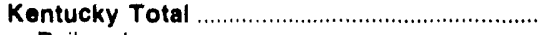 & 324 & - & 80 & $\bullet$ & 384 \\
\hline 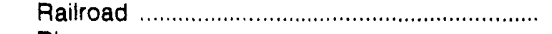 & 31 & - & 2 & - & 33 \\
\hline 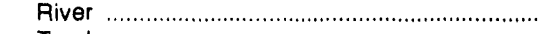 & 293 & - & 51 & - & 344 \\
\hline 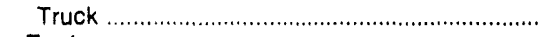 & - & - & 8 & • & 8 \\
\hline 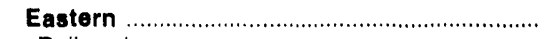 & 31 & - & 60 & - & 91 \\
\hline 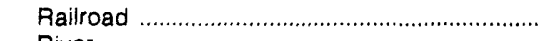 & 31 & - & 2 & - & 33 \\
\hline 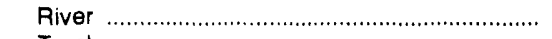 & - & - & 51 & - & 51 \\
\hline 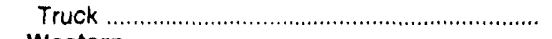 & - & - & 8 & - & 8 \\
\hline 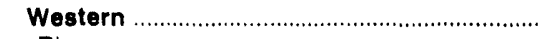 & 293 & - & - & * & 293 \\
\hline 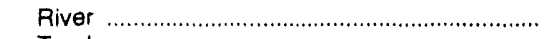 & 293 & - & - & - & 293 \\
\hline 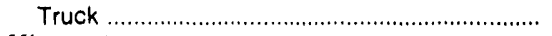 & - & - & - & - & • \\
\hline 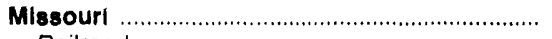 & 920 & - & 5 & 67 & 992 \\
\hline 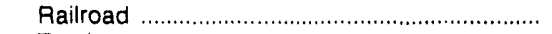 & 10 & - & - & - & 10 \\
\hline 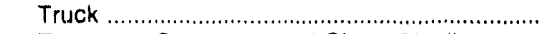 & 89 & - & 5 & 67 & 161 \\
\hline Tramway, Conveyor, and Slurry Pipeline ........ & 821 & - & - & - & 821 \\
\hline 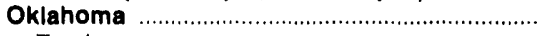 & - & - & - & * & * \\
\hline Truck & - & - & - & • & • \\
\hline 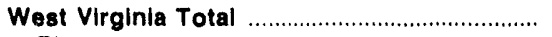 & - & - & 51 & 4 & 55 \\
\hline 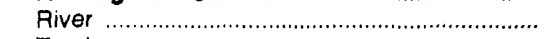 & - & - & 47 & 2 & 48 \\
\hline 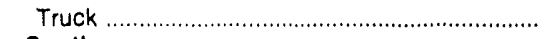 & - & - & 4 & 3 & 7 \\
\hline Southern & - & - & 51 & 4 & 55 \\
\hline 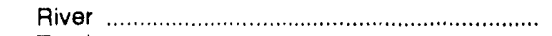 & - & - & 47 & 2 & 48 \\
\hline 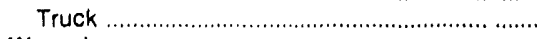 & - & - & 4 & 3 & 7 \\
\hline 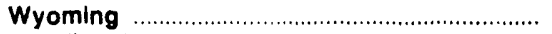 & 3,854 & - & - & - & 3,854 \\
\hline 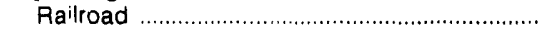 & 3,854 & - & - & - & 3,854 \\
\hline State Total & 11,409 & - & 533 & 136 & 12,077 \\
\hline 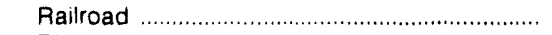 & 8,403 & - & 6 & - & 8,409 \\
\hline 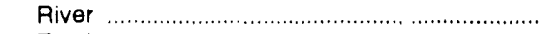 & 1,986 & - & 174 & 2 & 2,162 \\
\hline 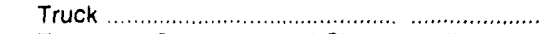 & 198 & - & 353 & 134 & 685 \\
\hline Tramway, Conveyor, and Slurry Pipeline ....... & 821 & - & - & - & 821 \\
\hline
\end{tabular}

See footnotes at end of table. 
Table 34. Domestic Distribution of U.S. Coal by Destination State, Consumer, Origin and Method of Transportation, January-June 1991 (Continued) (Thousand Short Tons)

\begin{tabular}{|c|c|c|c|c|c|}
\hline $\begin{array}{l}\text { Coal-Producing State of Origin } \\
\text { by Method of Transportation }\end{array}$ & $\begin{array}{l}\text { Electrlc } \\
\text { Utillities }\end{array}$ & $\begin{array}{l}\text { Coke } \\
\text { Plants }\end{array}$ & $\begin{array}{c}\text { Industrial } \\
\text { Plants } \\
\text { (Except Coke) }\end{array}$ & $\begin{array}{l}\text { Residential } \\
\text { and Commercial }\end{array}$ & Total \\
\hline & \multicolumn{5}{|c|}{ DESTINATION: MONTANA } \\
\hline Colorado . & - & - & 5 & - & 5 \\
\hline Railroad & - & - & 5 & - & 5 \\
\hline Illinole & 201 & - & - & - & 201 \\
\hline Railroad & 201 & - & - & - & 201 \\
\hline 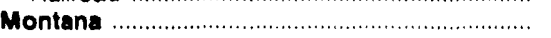 & 4,780 & - & 39 & 21 & 4,841 \\
\hline 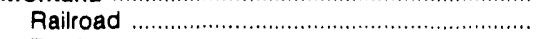 & 258 & - & 20 & - & 279 \\
\hline 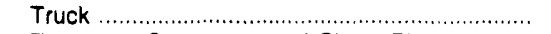 & 125 & - & 19 & 21 & 165 \\
\hline Tramway, Conveyor, and Slurry Pipeline ....... & 4,397 & - & - & - & 4,397 \\
\hline 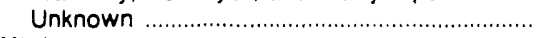 & - & - & $\cdot$ & - & 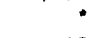 \\
\hline Utah & - & - & 19 & - & 19 \\
\hline 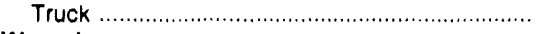 & - & - & 19 & - & 19 \\
\hline 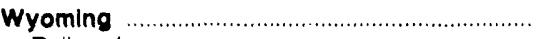 & - & - & 66 & " & 66 \\
\hline 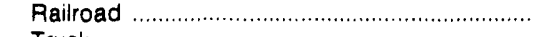 & - & - & 66 & - & 66 \\
\hline 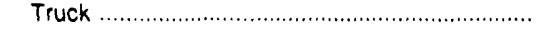 & - & - & - & • & • \\
\hline State Total & 4,982 & - & 130 & 21 & 5,132 \\
\hline Railroad & 460 & - & 91 & - & 551 \\
\hline 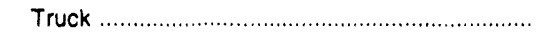 & 125 & - & 38 & 21 & 184 \\
\hline Tramway, Conveyor, and Slurry Pipeline ....... & 4,397 & - & - & - & 4,397 \\
\hline \multirow[t]{2}{*}{ 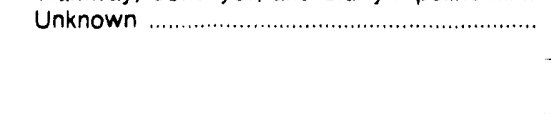 } & - & - & $\cdot$ & - & $\cdot$ \\
\hline & \multicolumn{5}{|c|}{ DESTINATION: NEBRASKA } \\
\hline 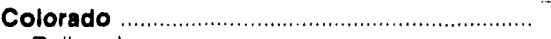 & - & - & 29 & 7 & 36 \\
\hline Raitroad & - & - & 29 & 7 & 36 \\
\hline 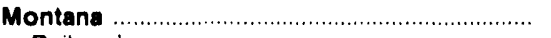 & - & - & 36 & - & 36 \\
\hline 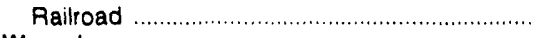 & - & - & 36 & - & 36 \\
\hline 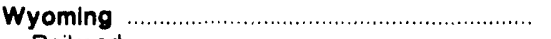 & 4,206 & - & - & - & 4,206 \\
\hline 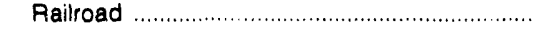 & 4,206 & - & - & - & 4,206 \\
\hline \multirow{3}{*}{ 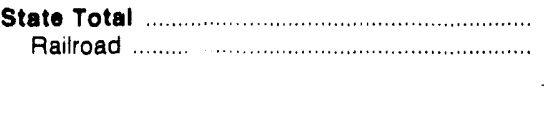 } & 4,206 & - & 65 & 7 & 4,278 \\
\hline & 4,206 & - & 65 & 7 & 4,278 \\
\hline & \multicolumn{5}{|c|}{ DESTINATION: NEVADA } \\
\hline Arizona & $2, \overline{575}$ & - & - & - & 2,575 \\
\hline Tramway, Conveyor, and Slurry Pipeline ....... & 2,575 & - & - & - & 2,575 \\
\hline Utah & 1,422 & - & 113 & 1 & 1,536 \\
\hline 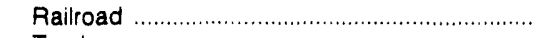 & 1,422 & - & 92 & - & 1.515 \\
\hline 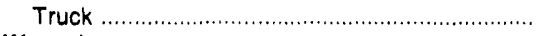 & - & - & 21 & 1 & 22 \\
\hline 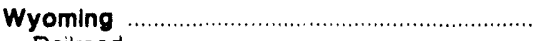 & 193 & - & - & - & 193 \\
\hline 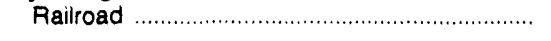 & 193 & - & - & - & 193 \\
\hline 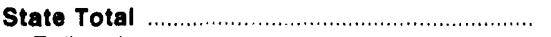 & 4,190 & - & 113 & 1 & 4,305 \\
\hline Railroad & 1,615 & - & 92 & - & 1,708 \\
\hline 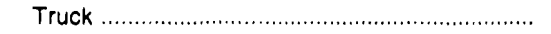 & - & - & 21 & 1 & 22 \\
\hline \multirow[t]{2}{*}{ Tramway, Conveyor, and Slurry Pipeline ....... } & 2,575 & - & - & - & 2,575 \\
\hline & \multicolumn{5}{|c|}{ DESTINATION: NEW HAMPSHIRE } \\
\hline 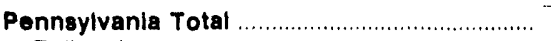 & 394 & - & 1 & 6 & 401 \\
\hline 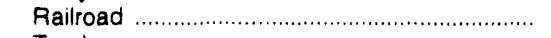 & 394 & - & 1 & 2 & 397 \\
\hline 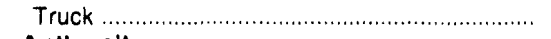 & - & - & - & 5 & 5 \\
\hline Anthraclte & - & - & " & 6 & 6 \\
\hline 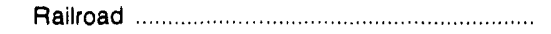 & - & - & • & 2 & 2 \\
\hline 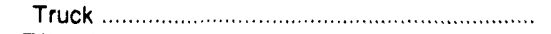 & - & - & - & 4 & 4 \\
\hline 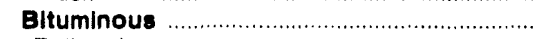 & 394 & - & 1 & • & 396 \\
\hline 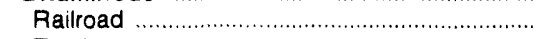 & 394 & - & 1 & - & 395 \\
\hline 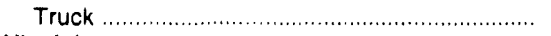 & - & - & - & " & - \\
\hline Virginia & 186 & - & - & - & 186 \\
\hline 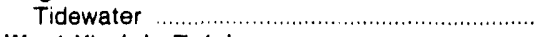 & 186 & - & - & - & 186 \\
\hline 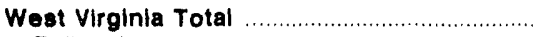 & 123 & - & - & - & 123 \\
\hline 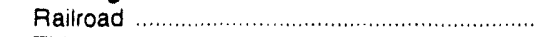 & 80 & $\sim$ & - & - & 80 \\
\hline 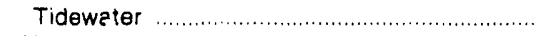 & 43 & - & - & - & 43 \\
\hline Northern . . & 80 & - & - & - & 80 \\
\hline 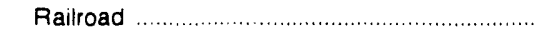 & 80 & - & - & - & 80 \\
\hline
\end{tabular}

See footnotes at end of table. 
Table 34. Domestic Distribution of U.S. Coal by Destination State, Consumer, Origin and Method of Transportation, January-June 1991 (Continued) (Thousand Short Tons)

\begin{tabular}{|c|c|c|c|c|c|}
\hline $\begin{array}{l}\text { Coal-Producing State of Origin } \\
\text { by Method of Transportation }\end{array}$ & $\begin{array}{l}\text { Electric } \\
\text { Utilities }\end{array}$ & $\begin{array}{l}\text { Coke } \\
\text { Plants }\end{array}$ & $\begin{array}{c}\text { Industrial } \\
\text { Plants } \\
\text { (Except Coke) }\end{array}$ & $\begin{array}{l}\text { Residential } \\
\text { and Commercial }\end{array}$ & Total \\
\hline & \multicolumn{5}{|c|}{ DESTINATION: NEW HAMPSHIRE (Continued) } \\
\hline Southern ............ & 43 & - & - & - & 43 \\
\hline Tidewater .. .. & 43 & - & - & - & 43 \\
\hline State Total & 703 & - & 1 & 6 & 710 \\
\hline Tidewater $\ldots \ldots \ldots \ldots \ldots \ldots \ldots \ldots$ & 229 & - & - & - & 229 \\
\hline \multirow[t]{2}{*}{ Truck $\ldots \ldots \ldots \ldots$} & - & - & - & 5 & 5 \\
\hline & \multicolumn{5}{|c|}{ DESTINATION: NEW JERSEY } \\
\hline Kentucky Total $\ldots$ & 17 & - & 12 & - & 29 \\
\hline Ralload & 17 & - & 12 & - & 29 \\
\hline Eastern & 17 & - & 12 & - & 29 \\
\hline$\ldots \ldots \ldots \ldots \ldots \ldots \ldots$ & 17 & - & 12 & - & 29 \\
\hline Truck & - & - & 4 & 4 & 8 \\
\hline Anthracite $\ldots \ldots \ldots \ldots \ldots \ldots$ & - & - & 4 & 4 & 8 \\
\hline Ralload & - & - & • & • & $\cdot$ \\
\hline Truck $\ldots \ldots \ldots \ldots \ldots$ & - & - & 4 & 4 & 8 \\
\hline Bituminous $\ldots \ldots$ & 8 & - & - & - & 8 \\
\hline Raitroad $\ldots \ldots \ldots \ldots$ & 8 & - & - & - & 8 \\
\hline Truck $\ldots \ldots \ldots \ldots$ & - & - & $\cdot$ & * & $\cdot$ \\
\hline Virginia & 210 & - & - & - & 210 \\
\hline 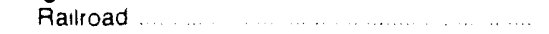 & 15 & - & - & - & 15 \\
\hline 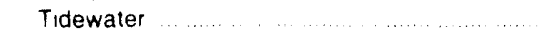 & 195 & - & - & - & 195 \\
\hline West Virginia Total & 596 & - & 124 & - & 720 \\
\hline Raltroc & 359 & - & 124 & - & 484 \\
\hline River $\ldots \ldots \ldots$ & 41 & - & - & - & 41 \\
\hline Tidewater $\ldots \ldots \ldots \ldots \ldots$ & 196 & - & - & - & 196 \\
\hline Northern $\ldots \ldots \ldots$ & 489 & - & 119 & - & 608 \\
\hline Railroad. & 346 & - & 119 & - & 465 \\
\hline River $\ldots \ldots$ & 41 & - & - & - & 41 \\
\hline 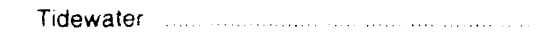 & 102 & - & - & - & 102 \\
\hline Southern $\ldots$ & 107 & - & 6 & - & 112 \\
\hline State Total $\ldots \ldots \ldots \ldots$ & 831 & - & 140 & 4 & 975 \\
\hline Railroad & 399 & - & 136 & $\cdot$ & 536 \\
\hline River & 41 & - & - & - & 41 \\
\hline 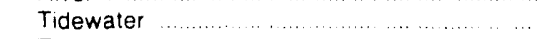 & 391 & - & - & - & 391 \\
\hline \multirow[t]{2}{*}{ Truck $\ldots \ldots \ldots$} & - & - & 4 & 4 & 8 \\
\hline & \multicolumn{5}{|c|}{ DESTINATION: NEW MEXICO } \\
\hline Colorado & 8 & - & 22 & - & 30 \\
\hline Truck & 8 & - & 22 & - & 30 \\
\hline New Mexico & 5,765 & - & - & 4 & 5,769 \\
\hline 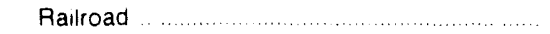 & 410 & - & - & - & 410 \\
\hline Truck $\ldots \ldots \ldots$ & - & - & - & 3 & 3 \\
\hline Tramway, Conveyor, and Slurry Pipeline $\ldots . .$. & 5.355 & - & - & - & 5,355 \\
\hline 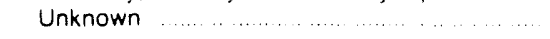 & - & - & - & * & $\cdot$ \\
\hline State Total $\ldots \ldots$ & 5,774 & - & 22 & 4 & 5,799 \\
\hline Railroad & 410 & - & 2 & - & 410 \\
\hline Truck $\ldots \ldots \ldots \ldots$ & 8 & - & 22 & 3 & 33 \\
\hline Tramway, Conveyor and Slurry Pipeline & 5,355 & - & - & - & 5.355 \\
\hline \multirow[t]{2}{*}{ Unknown $\quad \ldots \ldots$} & - & - & - & $\cdot$ & ? \\
\hline & \multicolumn{5}{|c|}{ DESTINATION: NEW YORK } \\
\hline Kentucky Total & 768 & 4 & 12 & 17 & 801 \\
\hline Railroad & 768 & 4 & 11 & 7 & 790 \\
\hline River & - & - & - & 9 & 9 \\
\hline Great Lakes & & - & - & 1 & 1 \\
\hline Truck & - & -. & • & - & - \\
\hline
\end{tabular}

See footnotes at end of table 
Table 34. Domestic Distribution of U.S. Coal by Destination State, Consumer, Origin and Method of Transportation, Jar.dary-June 1991 (Continued) (Thousand Short Tons)

\begin{tabular}{|c|c|c|c|c|c|}
\hline $\begin{array}{l}\text { Coal-Producing State of Origin } \\
\text { by Method of Transportation }\end{array}$ & $\begin{array}{l}\text { Electric } \\
\text { Utilitios }\end{array}$ & $\begin{array}{l}\text { Coke } \\
\text { Plante }\end{array}$ & $\begin{array}{c}\text { Industrial } \\
\text { Plants } \\
\text { (Except Coke) }\end{array}$ & $\begin{array}{c}\text { Residential } \\
\text { and Commercial }\end{array}$ & Total \\
\hline$\ldots$ & \multicolumn{5}{|c|}{ DESTINATION: NEW YORK (ContInued) } \\
\hline Eastern & 768 & 4 & 12 & 17 & 801 \\
\hline Railroad & 768 & 4 & 11 & 7 & 790 \\
\hline 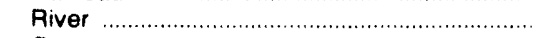 & - & - & - & 9 & 9 \\
\hline Great Lakes & - & - & - & 1 & 1 \\
\hline Truck & - & - & . & - & - \\
\hline 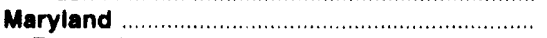 & 6 & - & - & - & 6 \\
\hline 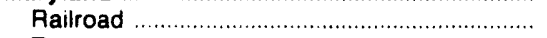 & 3 & - & - & - & 3 \\
\hline Truck & 3 & - & - & - & 3 \\
\hline 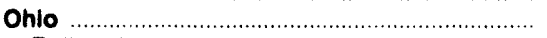 & $\cdot$ & - & 12 & 9 & 22 \\
\hline Railroad & - & - & 12 & - & 12 \\
\hline Great Lakes & $\cdot$ & - & - & • & . \\
\hline Truck & - & - & - & 9 & 9 \\
\hline 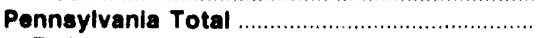 & 2,643 & 409 & 243 & 92 & 3,387 \\
\hline 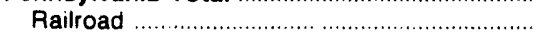 & 2,101 & 403 & 111 & 15 & 2,630 \\
\hline 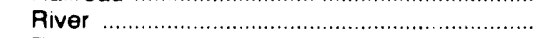 & 228 & - & 13 & - & 241 \\
\hline 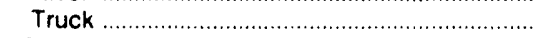 & 313 & 6 & 120 & 77 & 516 \\
\hline Anthracite & 1 & - & 7 & 47 & 56 \\
\hline Railroad & - & - & " & 8 & 8 \\
\hline Truck & 1 & - & 7 & 39 & 48 \\
\hline Bituminous & 2,642 & 409 & 236 & 44 & 3,331 \\
\hline Railroad & 2,101 & 403 & 111 & 7 & 2,621 \\
\hline 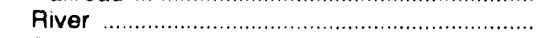 & 228 & - & 13 & - & 241 \\
\hline Truck & 312 & 6 & 112 & 38 & 468 \\
\hline Tennessee & - & - & - & 1 & 1 \\
\hline Railroad & - & - & - & 1 & 1 \\
\hline Virginia & - & - & 73 & - & 73 \\
\hline Railroad & - & - & 73 & - & 73 \\
\hline West Virginia Total & 1,638 & 58 & 489 & 9 & 2,195 \\
\hline Railroad & 1.633 & 58 & 434 & 4 & 2,129 \\
\hline River & - & - & 55 & - & 55 \\
\hline Great Lakes & 6 & - & - & - & 6 \\
\hline Truck & - & - & $\cdot$ & 5 & 5 \\
\hline Northern & 1,347 & - & 414 & 1 & 1,763 \\
\hline Railroad & 1,347 & - & 359 & - & 1.706 \\
\hline River & - & - & 55 & - & 55 \\
\hline Truck & - & - & 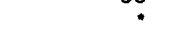 & 1 & 2 \\
\hline Southern & 292 & 58 & 75 & 8 & 432 \\
\hline Railroad & 286 & 58 & 75 & 4 & 423 \\
\hline Great Lakes & 6 & - & - & - & 6 \\
\hline Truck & - & - & - & 4 & 4 \\
\hline Wyoming & 9 & - & - & - & 9 \\
\hline Railroad & 9 & - & - & - & 9 \\
\hline State Total & 5,064 & 471 & 829 & 128 & 6,492 \\
\hline Railroad & 4,514 & 465 & 641 & 26 & 5,646 \\
\hline River & 228 & - & 68 & 9 & 306 \\
\hline Great Lakes & 6 & - & - & 1 & 7 \\
\hline \multirow{2}{*}{ 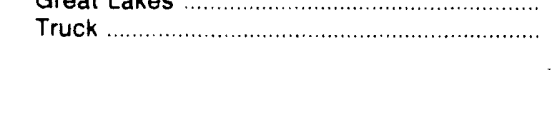 } & 317 & 6 & 120 & 91 & 534 \\
\hline & \multicolumn{5}{|c|}{ DESTINATION: NORTH CAROLINA } \\
\hline Kentucky Total & 4,754 & - & 778 & 14 & 5,546 \\
\hline Railroad & 4.754 & - & 624 & 12 & 5,390 \\
\hline 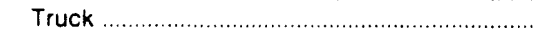 & - & - & 155 & 2 & 157 \\
\hline Eastern & 4,754 & - & 778 & 14 & 5,546 \\
\hline Railroad & 4,754 & - & 624 & 12 & 5.390 \\
\hline Truck & - & - & 155 & 2 & 157 \\
\hline Pennsylvania Total & - & - & 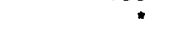 & $=$ & 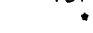 \\
\hline Railroad & - & - & • & - & • \\
\hline Anthracite & - & - & - & - & * \\
\hline Railroad & - & - & - & - & * \\
\hline Tennessee & - & - & 85 & - & 85 \\
\hline Railroad & - & - & 69 & - & 69 \\
\hline Truck & - & - & 16 & - & 16 \\
\hline 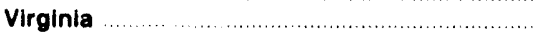 & 1,893 & - & 168 & 15 & 2,076 \\
\hline 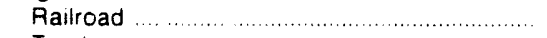 & 1,893 & - & 147 & 15 & 2,055 \\
\hline 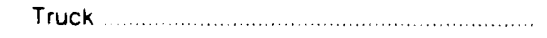 & - & - & 21 & - & 21 \\
\hline
\end{tabular}

See footnotes at end of table. 
Table 34. Domestic Distribution of U.S. Coal by Destination State, Consumer, Origin and Method of Transportation, January-June 1991 (Continued) (Thousand Short Tons)

\begin{tabular}{|c|c|c|c|c|c|}
\hline $\begin{array}{l}\text { Coal-Prod scing state of Origin } \\
\text { by Method of Traneportation }\end{array}$ & $\begin{array}{l}\text { Electric } \\
\text { Utilities }\end{array}$ & $\begin{array}{l}\text { Coke } \\
\text { Plants }\end{array}$ & $\begin{array}{c}\text { Industrial } \\
\text { Plants } \\
\text { (Except Coke) }\end{array}$ & $\begin{array}{l}\text { Residential } \\
\text { and Commercial }\end{array}$ & Total \\
\hline & \multicolumn{5}{|c|}{ DESTINATION: NORTH CAROLINA (Continued) } \\
\hline Weat Virginla Total & 2,564 & - & 206 & 18 & 2,788 \\
\hline Truck & - & - & 6 & - & 6 \\
\hline Northern & - & - & 2 & - & 2 \\
\hline Truck ……… & - & - & 2 & - & 2 \\
\hline Southern & 2,564 & - & 204 & 18 & 2,786 \\
\hline$Q_{\text {ailroad }}$ & 2.564 & - & 200 & 18 & 2.782 \\
\hline Truc: & - & - & 4 & - & 4 \\
\hline State Total & 9,211 & - & 1,238 & 47 & 10.495 \\
\hline Railroad & 9.211 & - & 1.040 & 45 & 10,295 \\
\hline \multirow[t]{2}{*}{ 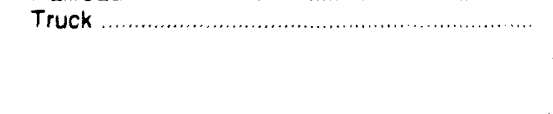 } & - & - & 198 & 2 & 200 \\
\hline & \multicolumn{5}{|c|}{ DESTINATION: NORTH DAKOTA } \\
\hline Kentucky Total. & - & - & 10 & - & 10 \\
\hline Railroad & - & - & 10 & - & 10 \\
\hline Eastern & - & - & 10 & - & 10 \\
\hline Railroad & - & - & 10 & - & 10 \\
\hline Montana & - & - & 129 & 4 & 133 \\
\hline 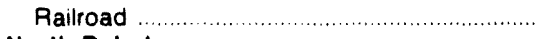 & - & - & 129 & 4 & 133 \\
\hline North Dakota & 10,287 & - & 2,969 & 70 & 13,326 \\
\hline Railroad & 913 & - & - & 12 & 925 \\
\hline Truck & 1,848 & - & 11 & 36 & 1,895 \\
\hline Tramway, Conveyor, and Slurry Pipeline ....... & 7,526 & - & 2.958 & 22 & 10,506 \\
\hline Unknown & - & - & - & 1 & 1 \\
\hline Pennaylvania Total & - & - & : & • & : \\
\hline Railroad & - & - & • & - & : \\
\hline 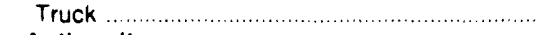 & - & - & - & • & : \\
\hline Anthracite & - & - & : & $*$ & * \\
\hline Railroad & - & - & • & $=$ & $\dot{0}$ \\
\hline Truck & - & - & - & $\cdot$ & • \\
\hline West Virginla Total & - & - & 1 & - & 1 \\
\hline Railroad & - & - & 1 & - & 1 \\
\hline Southern & - & - & 1 & - & $\vdots$ \\
\hline Raulroad & - & - & 1 & - & 1 \\
\hline Wyoming & - & - & - & : & $:$ \\
\hline Truck & - & - & - & $\cdot$ & * \\
\hline State Total. & 10,287 & - & 3,109 & 74 & 13,470 \\
\hline Ralload & 913 & - & 140 & 16 & 1.069 \\
\hline ………......... & 1,848 & - & 11 & 36 & 1,895 \\
\hline Tramway, Conveyor, and Slurry Pipeline ....... & 7,526 & - & 2,958 & 22 & 10,506 \\
\hline \multirow[t]{2}{*}{ 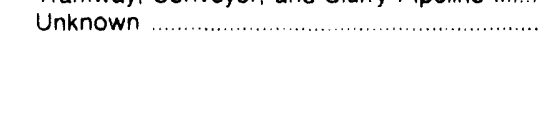 } & - & - & - & 1 & 1 \\
\hline & \multicolumn{5}{|c|}{ DESTINATION: OHIO } \\
\hline Colorado & 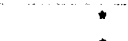 & - & - & - & • \\
\hline Raulroad & •" & - & - & - & . \\
\hline Illinols & - & - & : & - & : \\
\hline Truck & - & - & : & - & : \\
\hline Indlana & - & - & : & - & - \\
\hline Truck …… & - & - & $\dot{0}$ & $-\bar{c}$ & \\
\hline Kentucky Total & 4,378 & 252 & 929 & 60 & 5,619 \\
\hline 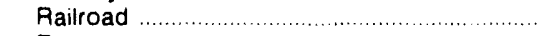 & 1,644 & 187 & 570 & 5 & 2,405 \\
\hline River & 2.660 & 56 & 14 & $\cdot$ & 2,730 \\
\hline Truck & 75 & 8 & 345 & 55 & 484 \\
\hline 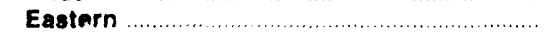 & 4,155 & 252 & 929 & 60 & 5,396 \\
\hline Railioad & 1,644 & 187 & 570 & 5 & 2,405 \\
\hline 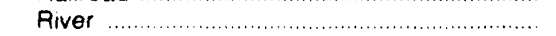 & 2,437 & 56 & 14 & • & 2,507 \\
\hline 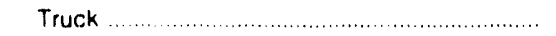 & 75 & 8 & 345 & 55 & 484 \\
\hline Western & 223 & - & - & - & 223 \\
\hline 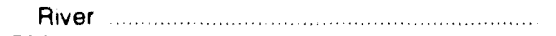 & 223 & - & - & - & 223 \\
\hline 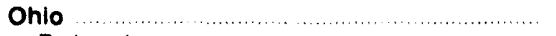 & 12,306 & - & 886 & 136 & 13,328 \\
\hline Raulroad & 1,091 & - & - & - & 1.091 \\
\hline River & 3,146 & - & 4 & - & 3,150 \\
\hline 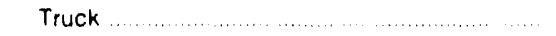 & 3,600 & - & 878 & 136 & 4.614 \\
\hline Tramway, Conveyor, and Slurry Pipeline & 4,468 & - & - & - & 4,468 \\
\hline Unknown & - & - & 5 & - & 5 \\
\hline
\end{tabular}

See footnotes at end of table. 
Table 34. Domestic Distribution of U.S. Coal by Destination State, Consumer, Origin and Method of Transportation, January-June 1991 (Continued) (Thousand Short Tons)

\begin{tabular}{|c|c|c|c|c|c|}
\hline $\begin{array}{l}\text { Coal-Producing State of Origin } \\
\text { by Method of Transportation }\end{array}$ & $\begin{array}{l}\text { Electric } \\
\text { Utilities }\end{array}$ & $\begin{array}{l}\text { Coke } \\
\text { Plants }\end{array}$ & $\begin{array}{c}\text { Industrial } \\
\text { Plants } \\
\text { (Except Coke) }\end{array}$ & $\begin{array}{c}\text { Residential } \\
\text { and Commerclal }\end{array}$ & Total \\
\hline & \multicolumn{5}{|c|}{ DESTINATION: OHIO (Continued) } \\
\hline Pennsylvanla Total. & 1,644 & - & 126 & $\cdot$ & 1,770 \\
\hline 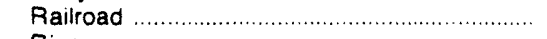 & 272 & - & $\cdot$ & - & 272 \\
\hline 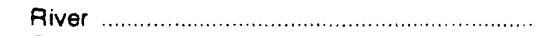 & 1.126 & - & - & - & 1.126 \\
\hline Great Lakes …………………… & 12 & - & 36 & - & 48 \\
\hline Truck & 234 & - & 90 & • & 324 \\
\hline 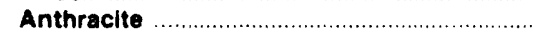 & - & - & 6 & • & 7 \\
\hline Railroad & - & - & : & . & : \\
\hline 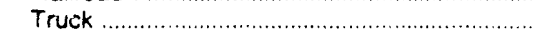 & - & - & 6 & • & 6 \\
\hline Bituminous & 1,644 & - & 120 & - & 1,764 \\
\hline Railroad & 272 & - & - & - & 272 \\
\hline 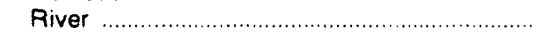 & 1,126 & - & - & - & 1,126 \\
\hline 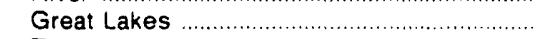 & 12 & - & 36 & - & 48 \\
\hline 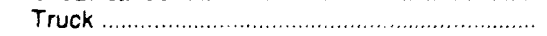 & 234 & - & 84 & . & 318 \\
\hline 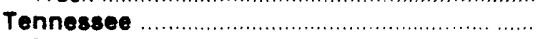 & - & - & - & 1 & 1 \\
\hline Truck & - & - & - & 1 & 1 \\
\hline 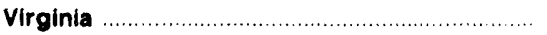 & - & 397 & 14 & - & 411 \\
\hline Railroad .................... & - & 290 & 14 & - & 304 \\
\hline 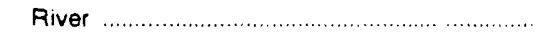 & - & 107 & - & - & 107 \\
\hline West Virginia Total & 6,986 & 1,084 & 387 & 28 & 8,485 \\
\hline 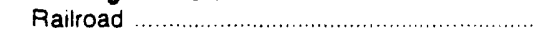 & 1,213 & 1.084 & 186 & 2 & 2,484 \\
\hline River & 5,590 & - & 47 & - & 5,637 \\
\hline Great Lakes & 132 & - & 30 & - & 162 \\
\hline Truck & 51 & - & 124 & 26 & 201 \\
\hline Northern & 2,934 & - & 37 & - & 2,971 \\
\hline Railroad ………… & 589 & - & - & - & 589 \\
\hline River & 2,252 & - & - & - & 2,252 \\
\hline Great Lakes & 90 & - & 30 & - & 120 \\
\hline Truck & 4 & - & 7 & - & 11 \\
\hline Southern & 4,053 & 1,084 & 349 & 28 & 5,514 \\
\hline Railroad & 624 & 1.084 & 186 & 2 & 1,895 \\
\hline River & 3,338 & - & 47 & - & 3,386 \\
\hline Great Lakes & 43 & - & - & - & 43 \\
\hline Truck & 47 & - & 116 & 26 & 190 \\
\hline 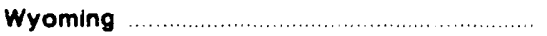 & 33 & - & - & - & 33 \\
\hline Railroad ……1. & 33 & - & - & - & 33 \\
\hline State Total & 25,346 & 1,732 & 2,342 & 226 & 29,647 \\
\hline 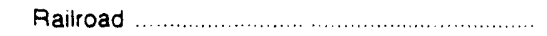 & 4.252 & 1,561 & 770 & 7 & 6,590 \\
\hline 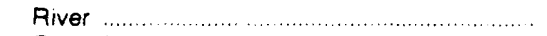 & 12,522 & 163 & 65 & • & 12,750 \\
\hline 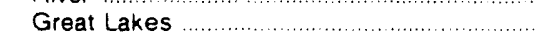 & 144 & - & 66 & - & 210 \\
\hline 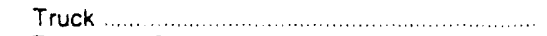 & 3,960 & 8 & 1,437 & 219 & 5,624 \\
\hline Tramway. Conveyor. and Slurry Pipeline ........ & 4,468 & - & - & - & 4,468 \\
\hline \multirow[t]{2}{*}{ Unknown } & - & - & 5 & " & 5 \\
\hline & \multicolumn{5}{|c|}{ DESTINATION: OKLAHOMA } \\
\hline Colorado & - & - & 57 & - & 57 \\
\hline Railroad & - & - & 57 & - & $5 i$ \\
\hline Indlana & - & - & 2 & - & 2 \\
\hline 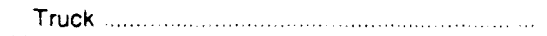 & - & - & 2 & - & 2 \\
\hline Oklahoma & 442 & - & 215 & - & 657 \\
\hline 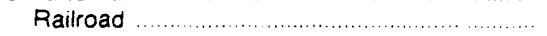 & 12 & - & - & - & 12 \\
\hline 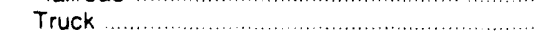 & 430 & - & 215 & - & 645 \\
\hline Unknown & - & - & - & - & . \\
\hline Pennsylvania Total & - & - & . & - & . \\
\hline Railroad & - & - & - & - & . \\
\hline Anthracite & - & - & - & - & . \\
\hline 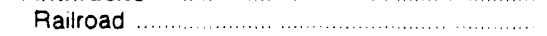 & - & - & - & - & . \\
\hline West Virginia Total & - & - & 9 & - & 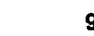 \\
\hline River & - & - & 9 & - & 9 \\
\hline Southern & - & - & 9 & - & 9 \\
\hline River & - & - & 9 & - & $\mathrm{s}$ \\
\hline 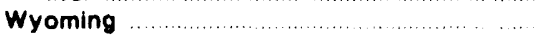 & 7,734 & - & 33 & - & 7,767 \\
\hline Rallroad & 7,734 & - & 33 & - & 7.767 \\
\hline State Total & 8,176 & - & 315 & $\cdot$ & 8,492 \\
\hline Railroad & 7.747 & - & 90 & - & 7.837 \\
\hline River & - & - & 9 & - & 9 \\
\hline
\end{tabular}

See footnotes at end of table 
Table 34. Domestic Distribution of U.S. Coal by Destination State, Consumer, Origin and Method of Transportation, January-June 1991 (Continued) (Thousand Short Tons)

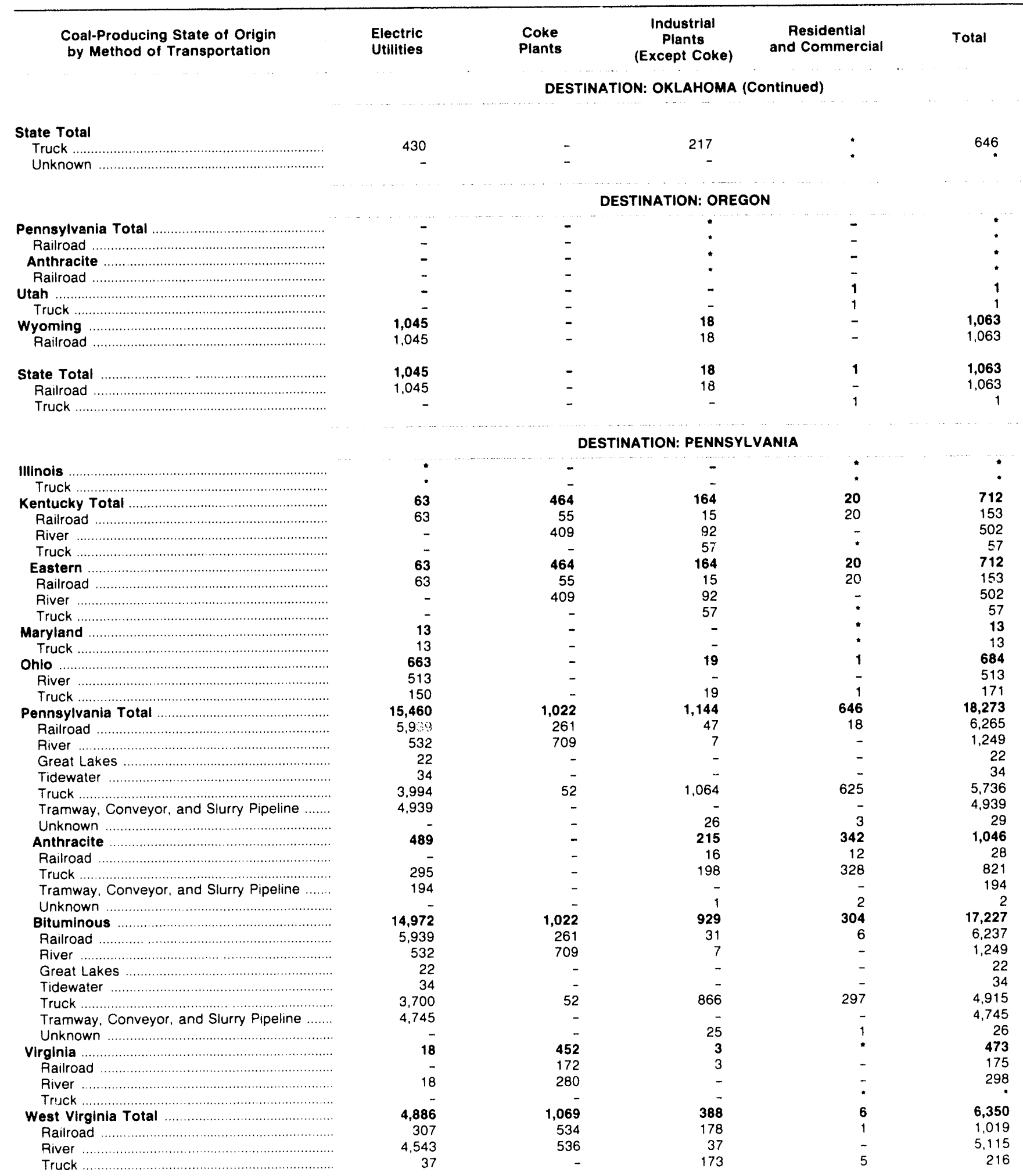

See footnotes at end of table 
Table 34. Domestic Distribution of U.S. Coal by Destination State, Consumer,
Origin and Method of Transportation, January-June 1991 (Continued) (Thousand Short Tons)

\begin{tabular}{|c|c|c|c|c|c|}
\hline & \multicolumn{5}{|c|}{ DESTINATION: PENNSYLVANIA (Continued) } \\
\hline 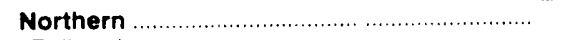 & 4,770 & - & 258 & 4 & 5,032 \\
\hline 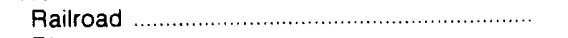 & 289 & - & 123 & - & 412 \\
\hline 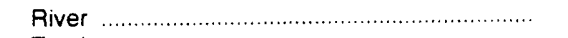 & 4,453 & - & - & - & 4,453 \\
\hline 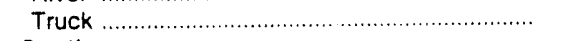 & 29 & - & 135 & 4 & 168 \\
\hline 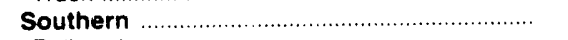 & 116 & 1,069 & 130 & 2 & 1,318 \\
\hline Railroad & 18 & 534 & 55 & 1 & 607 \\
\hline 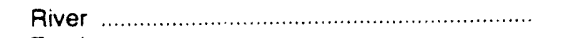 & 90 & 536 & 37 & - & 663 \\
\hline 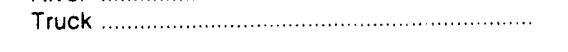 & 8 & - & 39 & 1 & 48 \\
\hline State Total & 21,104 & 3,007 & 1,719 & 675 & 26,505 \\
\hline 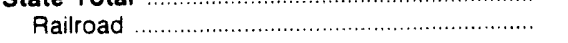 & 6,309 & 1,021 & 243 & 39 & 7,612 \\
\hline 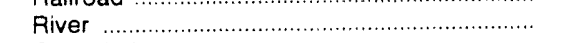 & 5,606 & 1.934 & 136 & - & 7,676 \\
\hline Great Lakes & 22 & - & - & - & 22 \\
\hline 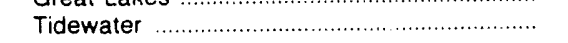 & 34 & - & - & - & 34 \\
\hline 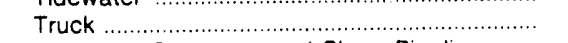 & 4.195 & 52 & 1,314 & 633 & 6,194 \\
\hline Tramway, Conveyor, and Slurry Pipeline ....... & 4,939 & - & - & - & 4,939 \\
\hline \multirow{2}{*}{ 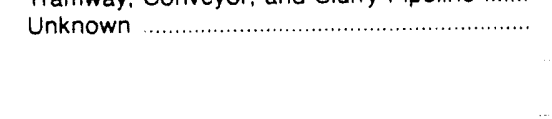 } & - & - & 26 & 3 & 29 \\
\hline & \multicolumn{5}{|c|}{ DESTINATION: RHODE ISI AND } \\
\hline Pennsylvania Total .............. & - & - & - & 1 & 1 \\
\hline Railroad & - & - & - & $\cdot$ & * \\
\hline 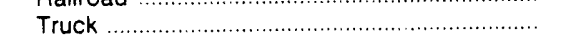 & - & - & - & 1 & 1 \\
\hline 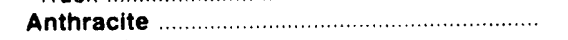 & - & - & - & 1 & 1 \\
\hline 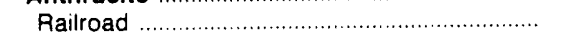 & - & - & - & $\cdot$ & : \\
\hline 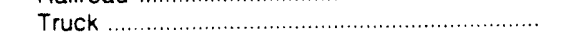 & - & - & - & 1 & 1 \\
\hline State Total ................... & - & - & - & 1 & 1 \\
\hline 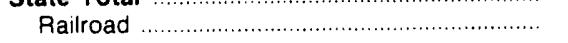 & - & - & - & : & $\cdot$ \\
\hline \multirow{2}{*}{ 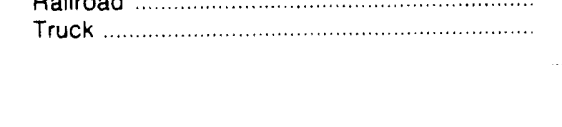 } & - & - & - & 1 & 1 \\
\hline & \multicolumn{5}{|c|}{ DESTINATION: SOUTH CAROLINA } \\
\hline Kentucky Total .................... & 3,785 & - & 669 & 1 & 4,455 \\
\hline 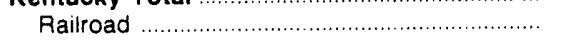 & 3,782 & - & 590 & 1 & 4,373 \\
\hline 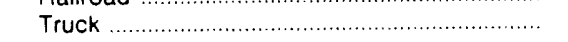 & 2 & - & 79 & 1 & 82 \\
\hline 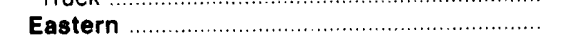 & 3,785 & - & 669 & 1 & 4,455 \\
\hline 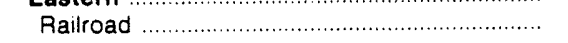 & 3,782 & - & 590 & 1 & 4,373 \\
\hline 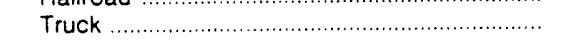 & 2 & - & 79 & 1 & 82 \\
\hline 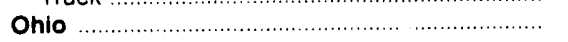 & - & - & 5 & - & 5 \\
\hline 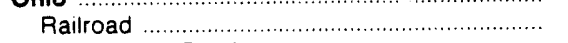 & - & - & 5 & - & 5 \\
\hline 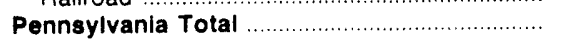 & - & - & 5 & $\cdot$ & 5 \\
\hline Railroad & - & - & • & $\cdot$ & . \\
\hline 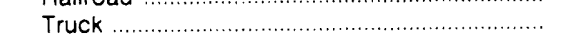 & - & - & 5 & - & 5 \\
\hline Anthracite & - & - & 3 & * & 3 \\
\hline 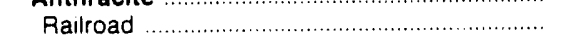 & - & - & * & • & 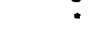 \\
\hline 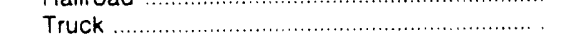 & - & - & 2 & - & 2 \\
\hline Bituminous & - & - & 2 & - & 2 \\
\hline Truck & - & - & 2 & - & 2 \\
\hline 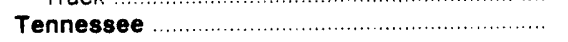 & - & - & 13 & 15 & 28 \\
\hline 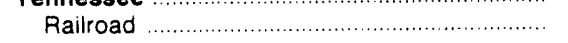 & - & - & 12 & 15 & 27 \\
\hline 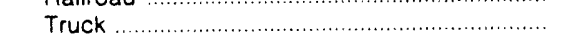 & - & - & 1 & 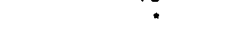 & 1 \\
\hline 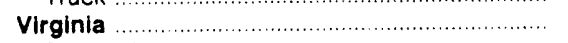 & 457 & - & 332 & - & 789 \\
\hline Railroad & 457 & - & 330 & - & 787 \\
\hline Truck & - & - & 2 & - & 2 \\
\hline West Virginia Total & 55 & - & 34 & - & 90 \\
\hline 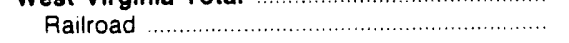 & 55 & - & 33 & - & 89 \\
\hline 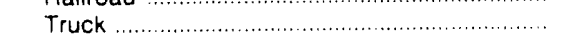 & - & - & 1 & - & 1 \\
\hline Southern & 55 & - & 34 & - & 90 \\
\hline 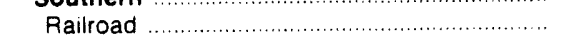 & 55 & - & 33 & - & 89 \\
\hline 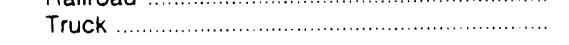 & - & - & 1 & - & 1 \\
\hline State Total & 4,297 & - & 1,058 & 16 & 5,371 \\
\hline 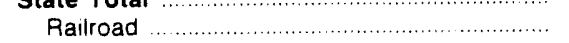 & 4.294 & - & 971 & 15 & 5,280 \\
\hline 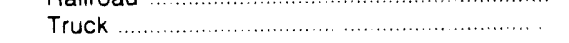 & 2 & - & 87 & 1 & 90 \\
\hline
\end{tabular}

See footnotes at end of table. 
Table 34. Domestic Distribution of U.S. Coal by Destination State, Consumer, Origin and Method of Transportation, January-June 1991 (Continued) (Thousand Short Tons)

\begin{tabular}{|c|c|c|c|c|c|}
\hline $\begin{array}{l}\text { Coal-Producing State of Origin } \\
\text { by Method of Transportation }\end{array}$ & $\begin{array}{l}\text { Electric } \\
\text { Utillties }\end{array}$ & $\begin{array}{l}\text { Coke } \\
\text { Plants }\end{array}$ & $\begin{array}{c}\text { Industrlal } \\
\text { Plants } \\
\text { (Except Coke) }\end{array}$ & $\begin{array}{c}\text { Residential } \\
\text { and Commercial }\end{array}$ & Total \\
\hline & \multicolumn{5}{|c|}{ DESTINATION: SOUTH DAKOTA } \\
\hline 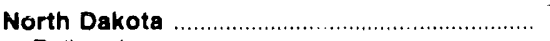 & 1,278 & - & - & . & 1,278 \\
\hline 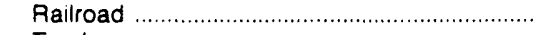 & 1,278 & - & - & - & 1,278 \\
\hline 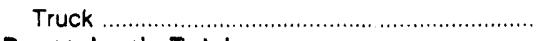 & .. & - & - & • & - \\
\hline 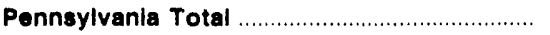 & - & - & * & - & * \\
\hline 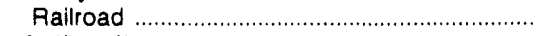 & - & - & * & - & * \\
\hline Anthracite & - & - & * & - & - \\
\hline 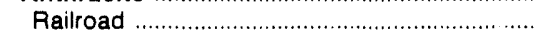 & - & - & - & - & - \\
\hline 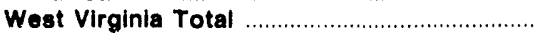 & - & - & 5 & - & 5 \\
\hline 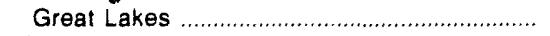 & - & - & 5 & - & 5 \\
\hline 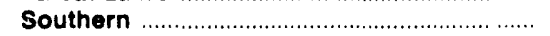 & - & - & 5 & - & 5 \\
\hline 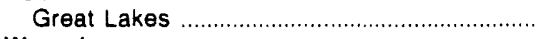 & - & - & 5 & - & 5 \\
\hline Wyoming & 127 & - & 98 & 2 & 227 \\
\hline 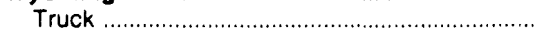 & 127 & - & 98 & 2 & 227 \\
\hline State Total & 1,406 & - & 103 & 2 & 1,510 \\
\hline Railroad & 1,278 & - & • & - & 1,278 \\
\hline 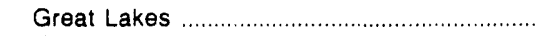 & - & - & 5 & - & 5 \\
\hline \multirow[t]{2}{*}{ 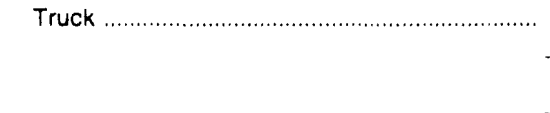 } & 127 & - & 98 & 2 & 227 \\
\hline & \multicolumn{5}{|c|}{ DESTINATION: TENNESSEE } \\
\hline Alabama & - & - & 3 & - & 3 \\
\hline 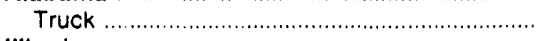 & - & - & 3 & - & 3 \\
\hline IIlinois & 664 & - & - & - & 664 \\
\hline River . & 664 & - & - & - & 664 \\
\hline Indiana & 113 & - & - & - & 113 \\
\hline 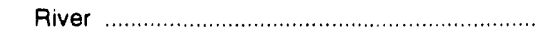 & 113 & - & - & - & 113 \\
\hline Kentucky Total & 7,859 & - & 938 & 41 & 8,839 \\
\hline 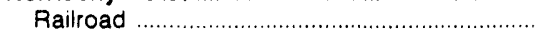 & 2,663 & - & 537 & - & 3,200 \\
\hline 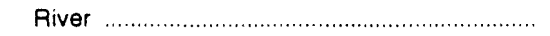 & 5,196 & - & 21 & 28 & 5,245 \\
\hline Truck & & - & 381 & 13 & 394 \\
\hline 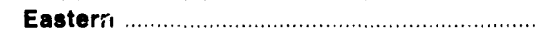 & 2,576 & - & 749 & 37 & 3,362 \\
\hline Railroad & 2,087 & - & 537 & - & 2,623 \\
\hline 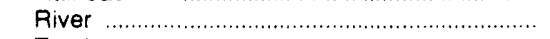 & 490 & - & 21 & 28 & 539 \\
\hline 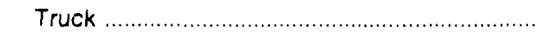 & & - & 191 & 8 & 200 \\
\hline Western & 5,283 & - & 189 & 5 & 5,477 \\
\hline 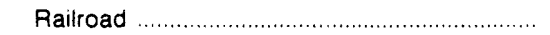 & 577 & - & - & - & 577 \\
\hline River & 4,706 & - & - & - & 4,706 \\
\hline Truck & - & - & 189 & 5 & 194 \\
\hline Ohlo & - & - & $*$ & - & : \\
\hline 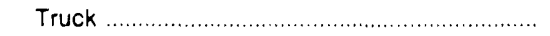 & - & - & - & - & • \\
\hline Pennsylvania Total & - & - & 3 & 6 & 9 \\
\hline Railroad & - & - & - & 6 & 6 \\
\hline Truck & - & - & 3 & - & 3 \\
\hline Anthraclte & - & - & 3 & 6 & 9 \\
\hline Railroad & - & - & • & 6 & 6 \\
\hline 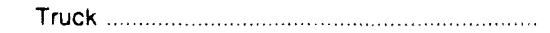 & - & - & 3 & - & 3 \\
\hline Tennessee & 802 & - & 150 & 56 & 1,008 \\
\hline 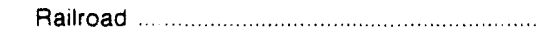 & 802 & - & - & 35 & 837 \\
\hline 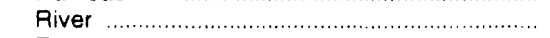 & - & - & - & 3 & 3 \\
\hline 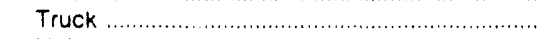 & - & - & 150 & 18 & 168 \\
\hline Unknown & - & - & - & • & - \\
\hline Virginla & 636 & - & 649 & 16 & 1,301 \\
\hline Railroad & 636 & - & 648 & 15 & 1.299 \\
\hline Truck & - & - & & 1 & 1 \\
\hline West Virginla Total & 551 & - & 33 & 8 & 592 \\
\hline 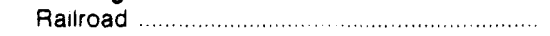 & - & - & 16 & - & 16 \\
\hline River & 551 & - & 17 & 2 & 570 \\
\hline 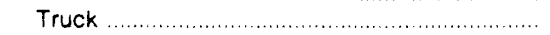 & - & - & - & 6 & 6 \\
\hline Northern & - & ـ & 14 & - & 14 \\
\hline River & - & - & 14 & - & 14 \\
\hline Southern & 551 & - & 19 & 8 & 579 \\
\hline 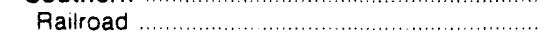 & - & - & 16 & - & 16 \\
\hline River & 551 & - & 3 & 2 & 556 \\
\hline 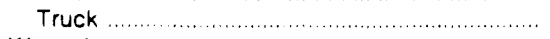 & & - & - & 6 & 6 \\
\hline Wyoming & 639 & - & - & - & 639 \\
\hline 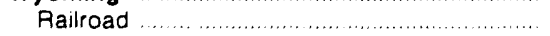 & $\iota 39$ & - & - & - & 639 \\
\hline
\end{tabular}

See footnctes at end of table 
Table 34. Domestic Distribution of U.S. Coal by Destination State, Consumer, Origin and Method of Transportation, January-June 1991 (Continued) (Thousand Short Tons)

\begin{tabular}{|c|c|c|c|c|c|}
\hline $\begin{array}{l}\text { Coaj-Producing State of Origin } \\
\text { by Method of Transportation }\end{array}$ & $\begin{array}{l}\text { Eloctric } \\
\text { Utilities }\end{array}$ & $\begin{array}{l}\text { Coke } \\
\text { Plante }\end{array}$ & $\begin{array}{c}\text { Industrial } \\
\text { Plants } \\
\text { (Except Coko) }\end{array}$ & $\begin{array}{l}\text { Rosidential } \\
\text { and Commerclal }\end{array}$ & Total \\
\hline & \multicolumn{5}{|c|}{ DESTINATION: TENNESSEE (Continued) } \\
\hline 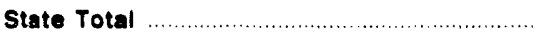 & 11,264 & - & 1,776 & 128 & 13,167 \\
\hline Railroad & 4.740 & - & 1,202 & 56 & 5,997 \\
\hline River & 6,524 & - & 37 & 33 & 6.594 \\
\hline 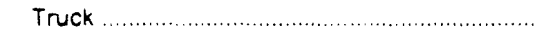 & & - & 536 & 39 & 575 \\
\hline \multirow[t]{2}{*}{ 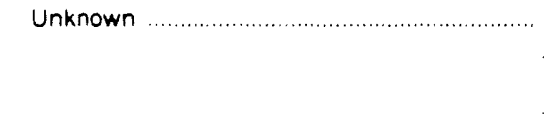 } & - & - & - & $\cdot$ & $\cdot$ \\
\hline & \multicolumn{5}{|c|}{ DESTINATION: TEXAS } \\
\hline$\ldots$ & - & - & $:$ & - & $\because$ \\
\hline 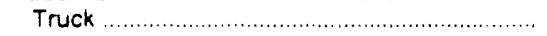 & - & - & • & - & $\cdot$ \\
\hline Colorado & 808 & - & 243 & - & 1,051 \\
\hline Railroad & 808 & - & 243 & - & 1.051 \\
\hline Illinols & • & - & - & - & . \\
\hline River & - & - & - & - & . \\
\hline Truck & • & - & - & - & $\cdot$ \\
\hline Indiana & - & - & - & • & $\cdot$ \\
\hline 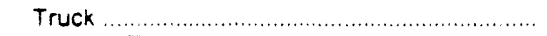 & - & - & - & • & $\cdot$ \\
\hline Kontucky Total ……. & - & - & 1 & - & 1 \\
\hline Rrver ........ & - & - & 1 & - & 1 \\
\hline Eastern & - & - & 1 & - & 1 \\
\hline River & - & - & 1 & - & 1 \\
\hline Now Mexico & - & - & 74 & - & 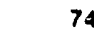 \\
\hline Rallroad & - & - & 74 & - & 74 \\
\hline Okiahoma & - & - & 77 & - & 77 \\
\hline Railroad & - & - & 77 & - & 77 \\
\hline Truck & - & - & - & - & $\cdot$ \\
\hline Ponnsylvania Total & - & - & 2 & - & 2 \\
\hline Ralload & - & - & 1 & - & 1 \\
\hline River & - & - & 2 & - & 2 \\
\hline Truck & - & - & • & - & . \\
\hline Anthracite & - & - & 1 & - & 1 \\
\hline Railroad & - & - & 1 & - & 1 \\
\hline 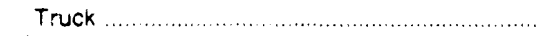 & - & - & - & - & . \\
\hline Bituminous & - & - & 2 & - & 2 \\
\hline River & - & - & 2 & - & 2 \\
\hline Truck & - & - & - & • & . \\
\hline Texas & 24,941 & - & 917 & - & 25,858 \\
\hline Rallroad & 10.570 & - & 144 & - & 10,716 \\
\hline 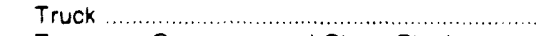 & 6.403 & - & 118 & - & 6.521 \\
\hline Tramway, Conveyor, and Slurry Pipeline $\ldots . . .$. & 7,968 & - & 655 & - & 8,623 \\
\hline Virginla & - & - & - & 6 & $\varepsilon$ \\
\hline Railruad & - & - & - & 6 & $\epsilon$ \\
\hline Wyoming & 17,467 & - & 109 & - & $17,57 \varepsilon$ \\
\hline Ralload & 17,467 & - & 109 & - & $17,57 \varepsilon$ \\
\hline Truck & - & - & - & - & \\
\hline State Total & 43,216 & - & 1,423 & 6 & 44,645 \\
\hline Railroad . . & 28,845 & - & 647 & 6 & 29.498 \\
\hline River & - & - & 3 & - & 3 \\
\hline Truck & 6.403 & - & 118 & - & 6,52 \\
\hline \multirow[t]{2}{*}{ Tramway, Conveyor, and Slury Pipeline ........ } & 7.968 & - & 655 & - & 8.623 \\
\hline & \multicolumn{5}{|c|}{ DESTINATION: UTAH } \\
\hline 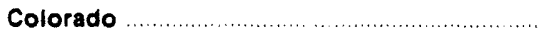 & 742 & 123 & $=$ & - & 865 \\
\hline Rallroad & 742 & 123 & - & - & 865 \\
\hline Ponnsylvanla Total $\ldots$ & - & 153 & - & - & 153 \\
\hline Railroad & - & 153 & - & - & 153 \\
\hline Anthracite & - & - & - & - & 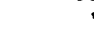 \\
\hline 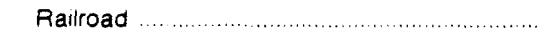 & - & - & - & - & ( \\
\hline Bituminous & - & 153 & - & - & 15 \\
\hline Railroad & - & 153 & - & - & 153 \\
\hline Utah & 5,935 & 301 & 278 & 161 & 6,67 \\
\hline Raliroad & 2,279 & 301 & • & - & 2,58 \\
\hline Truck & 2,462 & - & 276 & 160 & 2,89 \\
\hline Tramway, Conveyor, and Slurry Fipeline $\ldots . . . .$. & 1.194 & - & - & - & 1.19 \\
\hline Unknown & - & - & 1 & 2 & 3 \\
\hline
\end{tabular}

See footnotes at end of table 
Table 34. Domestic Distribution of U.S. Coal by Destination State, Consumer, Origin and Method of Transportation, January-June 1991 (Continued) (Thousand Short Tons)

\begin{tabular}{|c|c|c|c|c|c|}
\hline $\begin{array}{l}\text { Coal-Producing State of Origin } \\
\text { by Method of Transportation }\end{array}$ & $\begin{array}{l}\text { Electric } \\
\text { Utilities }\end{array}$ & $\begin{array}{l}\text { Coke } \\
\text { Plants }\end{array}$ & $\begin{array}{c}\text { Industrial } \\
\text { Plants } \\
\text { (Except Coke) }\end{array}$ & $\begin{array}{l}\text { Pesidential } \\
\text { and Commercial }\end{array}$ & Total \\
\hline Virginla & - & 58 & - & - & 58 \\
\hline 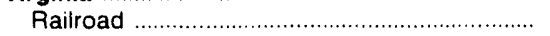 & - & 58 & - & - & 58 \\
\hline West Virginia Total & - & 35 & - & - & 35 \\
\hline Railroad & - & 35 & - & - & 35 \\
\hline Southern & - & 35 & - & - & 35 \\
\hline Railroad & - & 35 & - & - & 35 \\
\hline 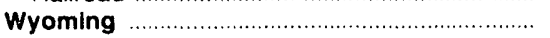 & 214 & - & 2 & - & 216 \\
\hline Railroad & 214 & - & 2 & - & 216 \\
\hline State Total & 6,891 & 669 & 279 & 161 & 8,001 \\
\hline Railroad & 3,235 & 669 & 2 & - & 3,907 \\
\hline Truck & 2,462 & - & 276 & 160 & 2,898 \\
\hline Tramway, Conveyor, and Slurry Pipeline ....... & 1,194 & - & - & - & 1,194 \\
\hline \multirow[t]{2}{*}{ 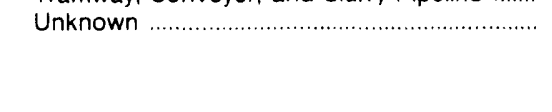 } & - & - & 1 & 2 & 3 \\
\hline & \multicolumn{5}{|c|}{ DESTINATION: VERMONT } \\
\hline Pennsylvania Total. & - & - & 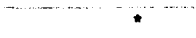 & 2 & 2 \\
\hline Railroad & - & - & - & 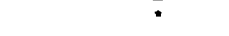 & " \\
\hline 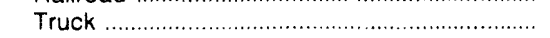 & - & - & • & 2 & 2 \\
\hline Anthracite & - & - & - & 2 & 2 \\
\hline 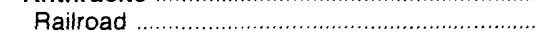 & - & - & - & 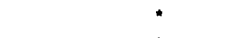 & * \\
\hline 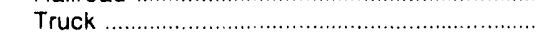 & - & - & - & 2 & 2 \\
\hline 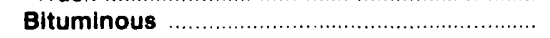 & - & - & * & - & : \\
\hline 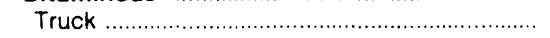 & - & - & • & - & * \\
\hline 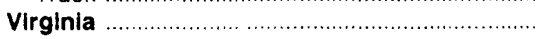 & - & - & 7 & - & 7 \\
\hline 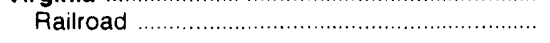 & - & - & 7 & - & 7 \\
\hline State Total & - & - & 7 & 2 & 9 \\
\hline 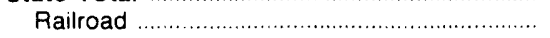 & - & - & 7 & 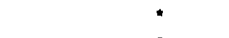 & 7 \\
\hline \multirow[t]{2}{*}{ 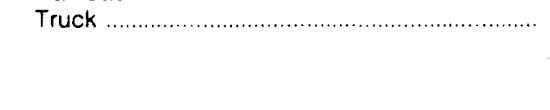 } & - & - & • & 2 & 2 \\
\hline & \multicolumn{5}{|c|}{ DESTINATION: VIRGINIA } \\
\hline (2) & 1,222 & - & 859 & 22 & 2,104 \\
\hline Railroad & 1,222 & - & 591 & 20 & 1,834 \\
\hline 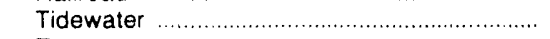 & - & - & 211 & - & 211 \\
\hline 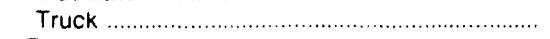 & - & - & 57 & 2 & 59 \\
\hline Eastern & 1,222 & - & 859 & 22 & 2,104 \\
\hline 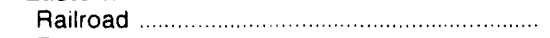 & 1,222 & - & 591 & 20 & 1,834 \\
\hline 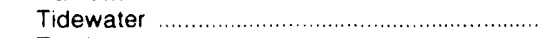 & - & - & 211 & - & 211 \\
\hline Truck & - & - & 57 & 2 & 59 \\
\hline 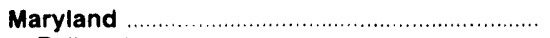 & 25 & - & - & - & 25 \\
\hline Railroad & 12 & - & - & - & 12 \\
\hline 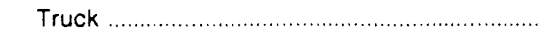 & 13 & - & - & - & 13 \\
\hline 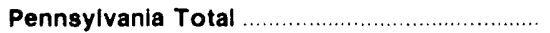 & 43 & - & 7 & * & 50 \\
\hline 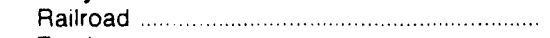 & 32 & - & * & - & 32 \\
\hline 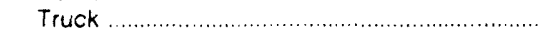 & 11 & - & 7 & * & 18 \\
\hline Anthracite & - & - & 7 & * & 7 \\
\hline Railroad & - & - & $\cdot$ & - & • \\
\hline Truck & - & - & 7 & * & 7 \\
\hline Bituminous & 43 & - & - & • & 43 \\
\hline Railroad & 32 & - & - & - & 32 \\
\hline 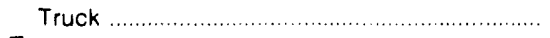 & 11 & - & - & • & 11 \\
\hline Tennessee & - & - & 8 & - & 8 \\
\hline 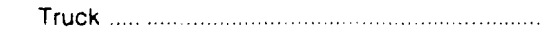 & - & - & 8 & - & 8 \\
\hline Virginia & 1,600 & 480 & 452 & 7 & 12,539 \\
\hline Railroad & 1,600 & - & 427 & 3 & 2,030 \\
\hline Truck & - & - & 13 & 2 & 15 \\
\hline Tramway, Conveyor, and Slurry Pipeline ....... & - & 480 & - & - & 480 \\
\hline 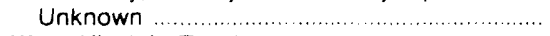 & - & - & 13 & 3 & 116 \\
\hline West Virginia Total & 1,768 & - & 504 & 34 & 2,306 \\
\hline Railroad & 1.641 & - & 383 & 25 & 2,049 \\
\hline 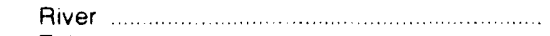 & - & - & - & 1 & 1 \\
\hline 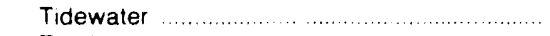 & 5 & - & 7 & - & 12 \\
\hline Truck & 122 & - & 114 & 8 & 244 \\
\hline
\end{tabular}

See footnotes at end of table. 
Table 34. Domestic Distribution of U.S. Coal by Destination State, Consumer, Origin and Method of Transportation, January-June 1991 (Continued) (Thousand Short Tons)

\begin{tabular}{|c|c|c|c|c|c|}
\hline $\begin{array}{l}\text { Coal-Producing State of Origin } \\
\text { by Method of Transportation }\end{array}$ & $\begin{array}{l}\text { Electric } \\
\text { Utilities }\end{array}$ & $\begin{array}{l}\text { Coke } \\
\text { Plants }\end{array}$ & $\begin{array}{c}\text { Industrial } \\
\text { Plants } \\
\text { (Except Coke) }\end{array}$ & $\begin{array}{l}\text { Residential } \\
\text { and Commercial }\end{array}$ & Total \\
\hline & \multicolumn{5}{|c|}{ DESTINATION: VIRGINIA (Continued) } \\
\hline Northern $\ldots \ldots \ldots \ldots \ldots \ldots$ & 180 & - & 22 & - & 202 \\
\hline Railroad $\ldots \ldots \ldots \ldots$ & 58 & - & - & - & 58 \\
\hline Truck & 122 & - & 22 & - & 144 \\
\hline 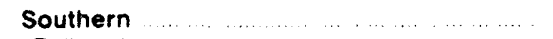 & 1.588 & - & 482 & 34 & 2,104 \\
\hline Railroad $\ldots$ & 1.583 & - & 383 & 25 & 1.991 \\
\hline 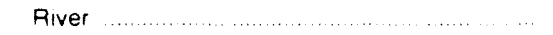 & - & - & - & 1 & 1 \\
\hline 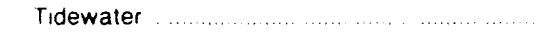 & 5 & - & 7 & - & 12 \\
\hline 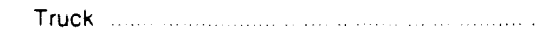 & - & - & 92 & 8 & 100 \\
\hline State Total & 4,658 & 480 & 1,830 & 64 & 17,031 \\
\hline 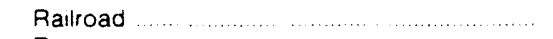 & 4.507 & - & 1,402 & 48 & 5.956 \\
\hline River .... & - & - & - & 1 & 1 \\
\hline 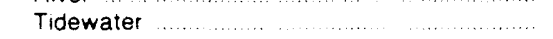 & 5 & - & 217 & - & 222 \\
\hline 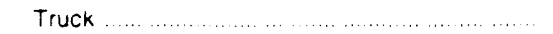 & 146 & - & 199 & 12 & 356 \\
\hline Tramway, Conveyor, and Slurry Pipeline & - & 480 & - & - & 480 \\
\hline \multirow[t]{2}{*}{ 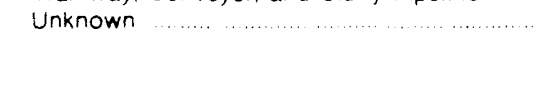 } & - & - & $: 3$ & 3 & 116 \\
\hline & \multicolumn{5}{|c|}{ DESTINATION: WASHINGTON } \\
\hline Colorado & - & - & - & 30 & 30 \\
\hline . & - & - & - & 30 & 30 \\
\hline 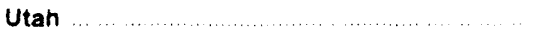 & - & - & 58 & 30 & 88 \\
\hline Railroad & - & - & 58 & 29 & 87 \\
\hline Truck & - & - & ? & 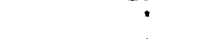 & $\bullet$ \\
\hline 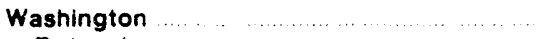 & 2,193 & - & - & $\cdot$ & 2,193 \\
\hline Ralroad $\ldots \ldots \ldots \ldots$ & 2.193 & - & - & - & 2.193 \\
\hline Truck $\ldots$ & 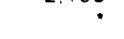 & - & - & $\cdot$ & 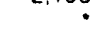 \\
\hline Wyoming & - & - & 23 & 1 & 24 \\
\hline 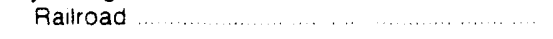 & - & - & 23 & 1 & 24 \\
\hline State Total & 2,193 & - & 81 & 80 & 2,334 \\
\hline Railroad & 2.193 & - & 81 & 60 & 2,333 \\
\hline \multirow[t]{2}{*}{ Truck } & $\cdot$ & - & $\cdot$ & 1 & 1 \\
\hline & \multicolumn{5}{|c|}{ DESTINATION: WEST VIRGINIA } \\
\hline Kentucky Tutal .... . . . . . . . . . . . & 313 & 111 & 214 & 7 & 646 \\
\hline 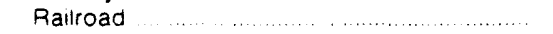 & - & - & 172 & 7 & 179 \\
\hline Fiver $\ldots \ldots \ldots \ldots$ & 301 & 111 & 9 & - & 421 \\
\hline 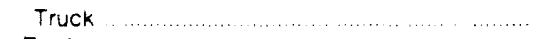 & 12 & - & 34 & - & 45 \\
\hline Easiern & 294 & 111 & 214 & 7 & 627 \\
\hline 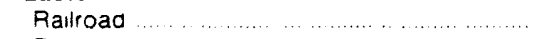 & - & - & 172 & 7 & 179 \\
\hline 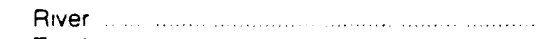 & 282 & 111 & 9 & - & 402 \\
\hline 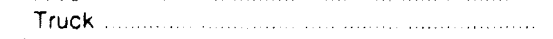 & 12 & - & 33 & • & 45 \\
\hline 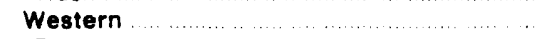 & 19 & - & 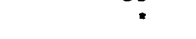 & - & 19 \\
\hline 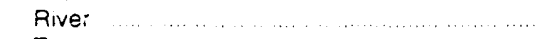 & 19 & - & - & - & 19 \\
\hline Truck $\ldots \ldots \ldots \ldots$ & - & - & $\cdot$ & - & $\cdot$ \\
\hline Maryland & 994 & - & - & * & 994 \\
\hline 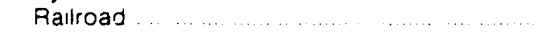 & 682 & - & - & - & 682 \\
\hline Truck & 313 & - & - & $\cdot$ & 313 \\
\hline Ohio $\ldots . .$. & 649 & - & 16 & - & 665 \\
\hline River $\ldots \ldots \ldots$ & 554 & - & - & - & 554 \\
\hline Truck $\ldots \ldots \ldots \ldots \ldots$ & 12 & - & 16 & - & 28 \\
\hline Tramway Conveyor and Slurry Pipeline ...... & 83 & - & - & - & 83 \\
\hline Pennsylvania Total & 499 & - & 276 & - & 775 \\
\hline 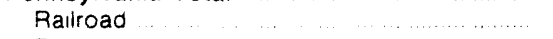 & 13 & - & 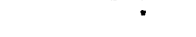 & - & 13 \\
\hline River $\quad \ldots \ldots$ & 403 & - & 146 & - & 549 \\
\hline Truck & 83 & - & 130 & - & 213 \\
\hline Anthracite & - & - & 4 & - & 4 \\
\hline 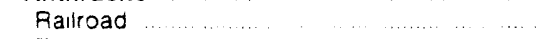 & - & - & ? & - & - \\
\hline Truck & - & - & 4 & $\cdot$ & 4 \\
\hline Bituminous $\ldots \ldots \ldots \ldots \ldots$ & 499 & - & 272 & - & 771 \\
\hline Raliroad $\ldots$ & 13 & - & - & - & 12 \\
\hline River $\quad \ldots \ldots \ldots \ldots$ & 403 & - & 146 & - & 549 \\
\hline Truck $\ldots \ldots \ldots \ldots$ & 83 & - & 126 & - & 209 \\
\hline$\ldots, \ldots, \ldots, \ldots \ldots, \ldots \ldots, \ldots \ldots$ & 7 & 408 & 2 & - & 417 \\
\hline Ral'road $\ldots \ldots$ & 7 & - & 1 & • & 8 \\
\hline River $\ldots \ldots \ldots, \ldots \ldots$ & - & 408 & - & - & 408 \\
\hline Truck $\ldots \ldots \ldots$ & - & - & 1 & - & 1 \\
\hline
\end{tabular}

See footnotes at end of table 
Table 34. Domestic Distribution of U.S. Coal by Destination State, Consumer, Origin and Method of Transportation, January-June 1991 (Continued) (Thousand Short Tons)

\begin{tabular}{|c|c|c|c|c|c|}
\hline $\begin{array}{l}\text { Coal-Producing State of Origin } \\
\text { by Method of Transportation }\end{array}$ & $\begin{array}{l}\text { Electric } \\
\text { Utillities }\end{array}$ & $\begin{array}{l}\text { Coke } \\
\text { Plants }\end{array}$ & $\begin{array}{c}\text { Industrial } \\
\text { Plants } \\
\text { (Except Coke) }\end{array}$ & $\begin{array}{c}\text { Residential } \\
\text { and Commerclal }\end{array}$ & Total \\
\hline & \multicolumn{5}{|c|}{ DESTINATION: WEST VIRGINIA (Continued) } \\
\hline West Virginia Total & 12,116 & 1,507 & 717 & 125 & 114,469 \\
\hline Railroad & 5,914 & 877 & 115 & - & 6,907 \\
\hline 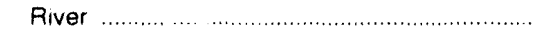 & 2,131 & 630 & 191 & - & 2,952 \\
\hline 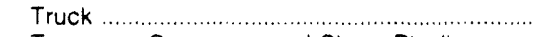 & 1,788 & - & 360 & 118 & 2,266 \\
\hline Tramway, Conveyor, and Slurry Pipeline ....... & 2,282 & - & - & - & 2,282 \\
\hline 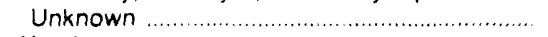 & - & - & 51 & 7 & 162 \\
\hline Northern & 7,454 & - & 224 & 114 & 7,792 \\
\hline 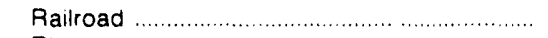 & 2,578 & - & 35 & - & 2,613 \\
\hline 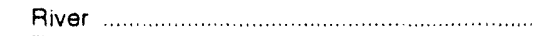 & 959 & - & 83 & - & 1,042 \\
\hline 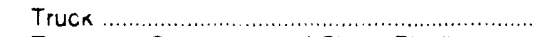 & 1,634 & - & 97 & 113 & 1,844 \\
\hline Tramway, Conveyor, and Slurry Pipeline ....... & 2,282 & - & - & - & 2,282 \\
\hline Unknown & - & - & 9 & 1 & 10 \\
\hline Southorn & 4,662 & 1,507 & 494 & 10 & 6,678 \\
\hline Railroad & 3,336 & 877 & 80 & - & 4,293 \\
\hline 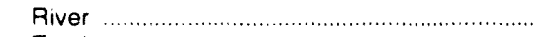 & 1,172 & 630 & 108 & - & 1,910 \\
\hline 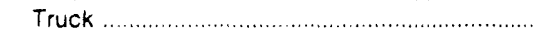 & 154 & - & 263 & 5 & 422 \\
\hline 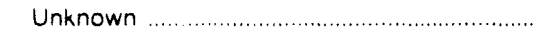 & - & - & 42 & 6 & 152 \\
\hline State Total & 14,578 & 2,026 & 1,226 & 132 & 117,967 \\
\hline 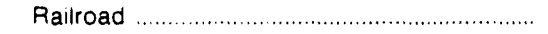 & 6,615 & 877 & 288 & 7 & 7,789 \\
\hline 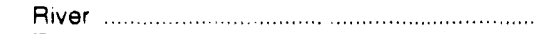 & 3,390 & 1,149 & 346 & - & 4,885 \\
\hline 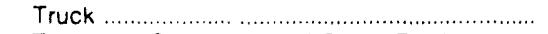 & 2,207 & - & 541 & 118 & 2,866 \\
\hline Tramway, Conveyor, and Slurry Pipeline ....... & 2,366 & - & - & - & 2,366 \\
\hline \multirow[t]{2}{*}{ Unknown } & - & - & 51 & 7 & 162 \\
\hline & \multicolumn{5}{|c|}{ DESTINATION: WISCONSIN } \\
\hline IIIInois $\ldots . . .$. & 227 & - & 153 & - & 380 \\
\hline (2) & 155 & - & 153 & - & 308 \\
\hline River & 72 & - & - & - & 72 \\
\hline Indiana & 1,141 & - & 36 & - & 1,177 \\
\hline Railroad & 861 & - & 30 & - & 890 \\
\hline River & 280 & - & - & - & $28 \mathrm{~J}$ \\
\hline 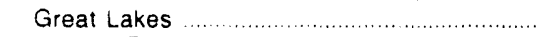 & - & - & 7 & - & 7 \\
\hline 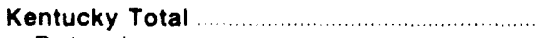 & 448 & - & 278 & 9 & $7: 15$ \\
\hline Railroad & 119 & - & 52 & - & 171 \\
\hline River & 254 & - & 13 & 9 & 276 \\
\hline 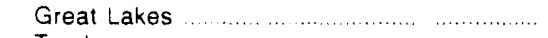 & 75 & - & 185 & - & 2.60 \\
\hline 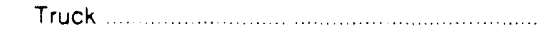 & - & - & 28 & - & 28 \\
\hline Eastern & 230 & - & 234 & 9 & 473 \\
\hline 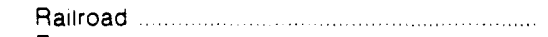 & 119 & - & 10 & - & 129 \\
\hline 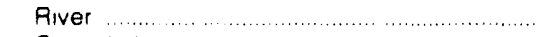 & 42 & - & 13 & 9 & 64 \\
\hline 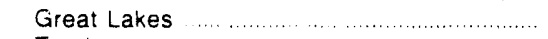 & 69 & - & 185 & - & 254 \\
\hline 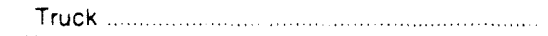 & - & - & 26 & - & 26 \\
\hline 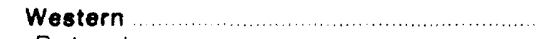 & 218 & - & 44 & - & 262 \\
\hline Railroad & - & - & 42 & - & 42 \\
\hline River & 212 & - & - & - & 212 \\
\hline Great Lakes & 6 & - & - & - & 6 \\
\hline 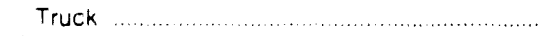 & - & - & 2 & - & 2 \\
\hline , & 918 & - & - & - & 918 \\
\hline 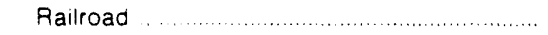 & 816 & - & - & - & 816 \\
\hline River & 102 & - & - & - & 102 \\
\hline New Mexico & 46 & - & - & - & 46 \\
\hline Railroad & 46 & - & - & - & 46 \\
\hline 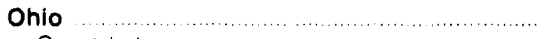 & 6 & - & - & - & 6 \\
\hline 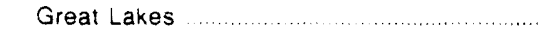 & 6 & - & - & - & 6 \\
\hline Pennsylvania Total & 726 & - & 26 & $\cdot$ & 753 \\
\hline Railroad & 726 & - & $\cdot$ & - & 727 \\
\hline Great Lakes & - & - & 26 & - & 26 \\
\hline Truck $\ldots \ldots \ldots$ & - & - & $\cdot$ & • & $\bullet$ \\
\hline Anthracite $\ldots \ldots \ldots$ & - & - & * & • & - \\
\hline Ralload $\ldots \ldots \ldots \ldots$ & - & - & • & - & • \\
\hline 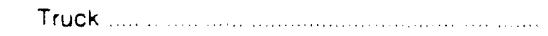 & - & - & $\cdot$ & $\cdot$ & • \\
\hline Bltuminous & 726 & - & 26 & - & 752 \\
\hline Railroad $\ldots \ldots \ldots$ & 726 & - & - & - & 726 \\
\hline Great Lakes $\ldots \ldots \ldots \ldots \ldots$ & - & - & 26 & - & 26 \\
\hline
\end{tabular}

See footnotes at end of table. 
Table 34. Domestic Distribution of U.S. Coal by Destination State, Consumer, Origin and Method of Transportation, January-June 1991 (Continued) (Thousand Short Tons)

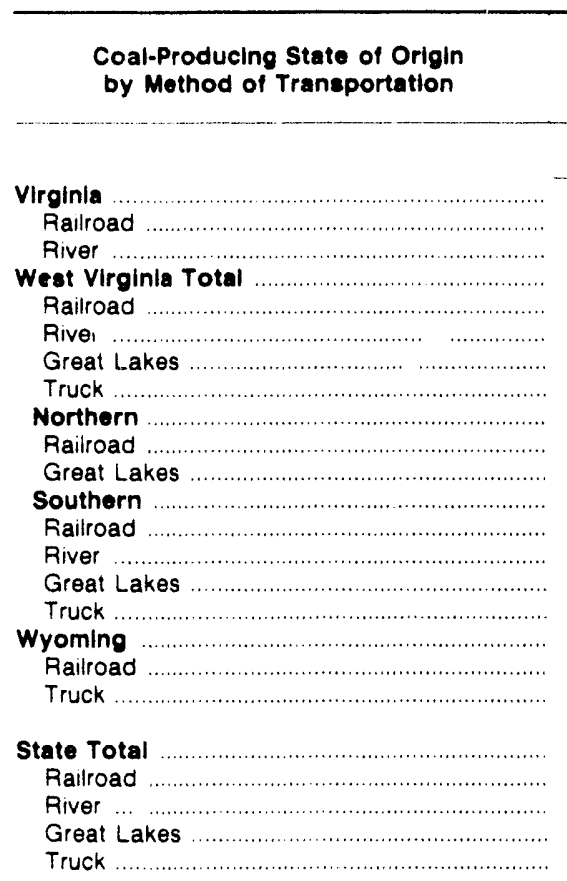

Electric
Utilltios

Industrial
Plants
(Except Coke)

Residential

and Commerclal

Total

DESTINATION: WISCONSIN (ContInUEd)

\begin{tabular}{|c|c|c|c|c|}
\hline 43 & - & - & & 43 \\
\hline $\begin{array}{l}21 \\
22\end{array}$ & - & - & :- & $\begin{array}{l}21 \\
22\end{array}$ \\
\hline $\begin{array}{l}28 \\
286\end{array}$ & $=$ & 79 & $=$ & $\begin{array}{l}22 \\
365\end{array}$ \\
\hline 270 & - & & - & 270 \\
\hline & - & 26 & - & 26 \\
\hline 17 & - & 44 & - & 61 \\
\hline 209 & - & $\begin{array}{r}9 \\
34\end{array}$ & - & $\begin{array}{r}9 \\
242\end{array}$ \\
\hline 209 & - & - & $=$ & $\begin{array}{l}242 \\
209\end{array}$ \\
\hline & - & 34 & - & \\
\hline $\begin{array}{l}78 \\
61\end{array}$ & $=$ & 45 & - & $\begin{array}{r}123 \\
61\end{array}$ \\
\hline & - & 26 & $=$ & 26 \\
\hline 17 & - & 10 & - & 27 \\
\hline $5,97 \overline{-}^{-}$ & - & $\begin{array}{r}9 \\
166\end{array}$ & - & 6,141 \\
\hline 5,959 & - & 166 & 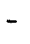 & \\
\hline 16 & - & 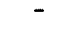 & - & \\
\hline 9,816 & - & 738 & 9 & $1 C, 564$ \\
\hline 8,972 & - & 400 & & \\
\hline 731 & - & 39 & 9 & \\
\hline 98 & - & 262 & & 360 \\
\hline 16 & - & 37 & - & 53 \\
\hline
\end{tabular}

DESTINATION: WYOMING

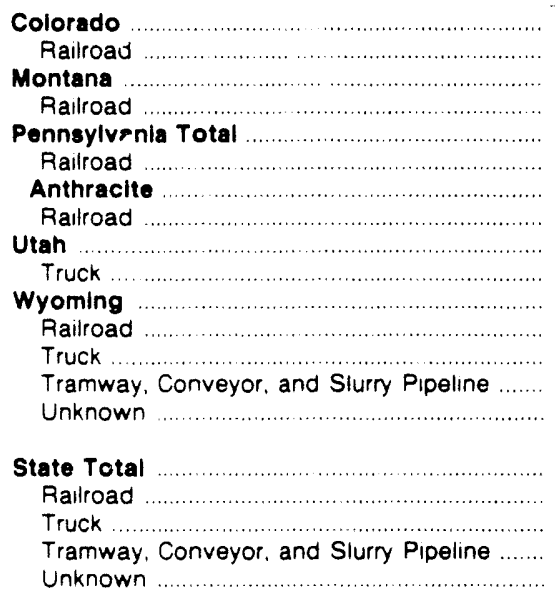

\begin{tabular}{|c|c|c|c|c|}
\hline \multicolumn{5}{|c|}{ DESTINATION: WYOMING } \\
\hline- & - & 32 & - & 32 \\
\hline - & - & 32 & - & 32 \\
\hline - & - & 3 & - & 3 \\
\hline- & - & 3 & - & 3 \\
\hline- & - & - & - & 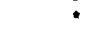 \\
\hline - & - & - & * & - \\
\hline - & - & - & * & - \\
\hline - & - & - & * & 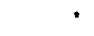 \\
\hline - & - & 35 & - & 35 \\
\hline - & - & 35 & - & 35 \\
\hline 11,179 & - & 787 & 63 & 12,029 \\
\hline 5,526 & - & 615 & 8 & 6,148 \\
\hline 865 & - & 162 & 55 & $1 . r 32$ \\
\hline 4,788 & - & - & - & 4,788 \\
\hline - & - & 9 & 1 & 10 \\
\hline 11,179 & - & 856 & 63 & 12,098 \\
\hline 5,526 & - & 649 & 8 & 6.183 \\
\hline 865 & - & 197 & 55 & 1.117 \\
\hline 4,788 & - & - & - & 4.788 \\
\hline - & - & 9 & 1 & 10 \\
\hline
\end{tabular}

\footnotetext{
Alabama

Railroad

River

Truck

Tramway, Conveyor, and Slurry Pipeline Unknown

Alaska

Raılroad$$
\text { Truck }
$$

Arizona

Railroad

Tramway. Conveyor, and Slurry Pipeline

Callfornia

Truck
}

DESTINATION: U.S. TOTAL

$\begin{array}{rrrrr}8,493 & 640 & 817 & 4 & 2 \\ 3,115 & 152 & 170 & - & 10,249 \\ 1,026 & - & 90 & - & 3,436 \\ 3,498 & 488 & 499 & 4 & 1.115 \\ 855 & - & - & - & 4,489 \\ - & - & 59 & - & 855 \\ 156 & - & - & 256 & 2354 \\ 76 & - & - & 252 & 412 \\ 80 & - & - & 4 & 328 \\ 6,362 & - & - & - & 84 \\ 3,787 & - & - & - & 6,362 \\ 2,575 & - & 8 & - & 3,787 \\ - & - & 8 & - & 2,575 \\ - & - & - & - & 8 \\ & - & - & & 8\end{array}$

See footnotes at end of table. 
Table 34. Domestic Distribution of U.S. Coal by Destination State, Consumer,
Origin and Method of Transportation, January-June 1991 (Continued) (Thousand Short Tons)

\begin{tabular}{|c|c|c|c|c|c|}
\hline \multirow[t]{2}{*}{$\begin{array}{l}\text { Coal-Producing State of Origin } \\
\text { by Method of Transportation }\end{array}$} & $\begin{array}{l}\text { Electric } \\
\text { Utilities }\end{array}$ & $\begin{array}{l}\text { Coke } \\
\text { lants }\end{array}$ & $\begin{array}{c}\text { Industria } \\
\text { Plants } \\
\text { (Except Coks) }\end{array}$ & $\begin{array}{c}\text { Residential } \\
\text { and Commercial }\end{array}$ & Total \\
\hline & \multicolumn{5}{|c|}{ DESTINATION: U.S. TOTAL (Continued) } \\
\hline Colorado & 7,761 & 123 & 819 & 64 & ${ }^{3} 8,768$ \\
\hline (n) & 5.571 & 123 & 691 & 36 & 6.422 \\
\hline … & 2.190 & - & 128 & 27 & 2.345 \\
\hline Unknown & - & - & - & 1 & 32 \\
\hline Illinois & 25,682 & 363 & 1,982 & 159 & 228,193 \\
\hline Railroad ........ & 13,919 & 363 & 632 & 13 & 14,926 \\
\hline (n) & 8,727 & - & 161 & - & 8,888 \\
\hline 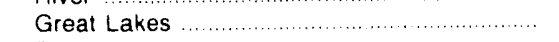 & 10 & - & - & - & 10 \\
\hline Truck ... & 1.790 & - & 1,177 & 144 & 3,111 \\
\hline Tramway, Conveyor, and Slurry Pipeline ....... & 1,237 & - & - & - & 1,237 \\
\hline 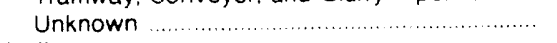 & - & - & 12 & 2 & 221 \\
\hline (1) & 12,505 & - & 1,947 & 242 & 214,818 \\
\hline 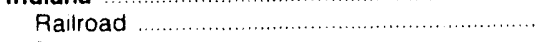 & 7,330 & - & 841 & - & 8,172 \\
\hline River & 2,792 & - & 308 & - & 3,099 \\
\hline 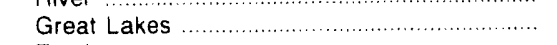 & 48 & - & 98 & - & 146 \\
\hline 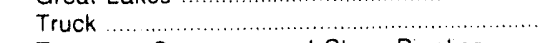 & 1,983 & - & 689 & 242 & 2,913 \\
\hline Tramway, Conveyor, and Slurry Pipeline ...... & 352 & - & - & - & 352 \\
\hline 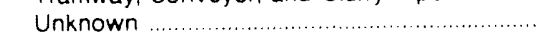 & - & - & 11 & 1 & 2135 \\
\hline lowa & 174 & - & - & 1 & 175 \\
\hline 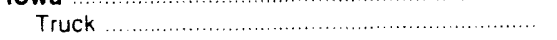 & 174 & - & - & 1 & 175 \\
\hline 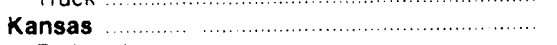 & 190 & - & 67 & 1 & 258 \\
\hline 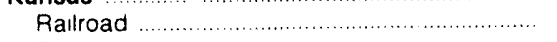 & 27 & - & - & - & 27 \\
\hline 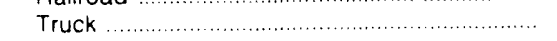 & 162 & - & 67 & 1 & 230 \\
\hline 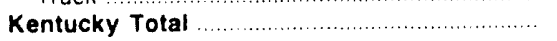 & 58,323 & 1,906 & 7,487 & 542 & 368,981 \\
\hline Railroad & 35,272 & 1,046 & 4.716 & 198 & 41,232 \\
\hline 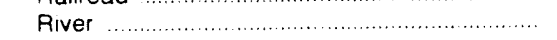 & 17,146 & 843 & 569 & 185 & 18,743 \\
\hline 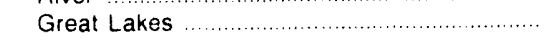 & 304 & - & 408 & 2 & 715 \\
\hline 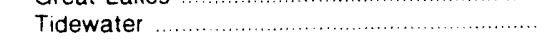 & 1,242 & - & 211 & - & 1,453 \\
\hline Truck & 3,084 & 17 & 1.576 & 119 & 4,796 \\
\hline Tramway, Conveyor, and Slurry Pipeline ....... & 1,275 & - & - & - & 1.275 \\
\hline 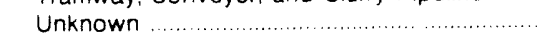 & - & - & 8 & 37 & 3768 \\
\hline (n) & 38,977 & 1,906 & 6,978 & 484 & 349,017 \\
\hline (1) & 30,532 & 1,046 & 4,666 & 198 & 36,442 \\
\hline (2. & 5,595 & 843 & 336 & 144 & 6,917 \\
\hline Great Lakes & 238 & - & 402 & 2 & 642 \\
\hline 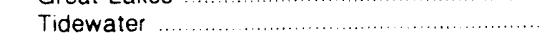 & 1,095 & - & 211 & - & 1.306 \\
\hline 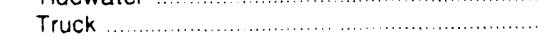 & 1,518 & 17 & 1.355 & 105 & 2,994 \\
\hline Unknown & - & - & 8 & 36 & 3715 \\
\hline (1) & $19,34 \mathrm{C}$ & - & 509 & 57 & 219,964 \\
\hline Railroad & 4,739 & - & 49 & 1 & 4,789 \\
\hline 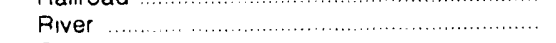 & 11,552 & - & 232 & 42 & 11,826 \\
\hline Great Lakes & 67 & - & 6 & - & 73 \\
\hline 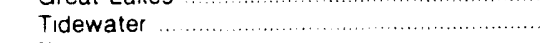 & 147 & - & - & - & 147 \\
\hline Truck & 1.566 & - & 221 & 14 & 1,802 \\
\hline Tramway. Conveyor, and Slurry Pipeline ...... & 1,275 & - & - & - & 1,275 \\
\hline 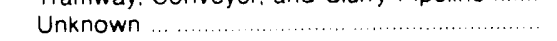 & - & - & - & 1 & 252 \\
\hline Louisiana & 1,287 & - & - & - & 1,287 \\
\hline Truck. & 177 & - & - & - & 177 \\
\hline Tramway. Conveyor, and Slurry Pipeline ...... & 1,111 & - & - & - & 1,111 \\
\hline Maryland & 1,464 & - & 184 & 2 & 21,655 \\
\hline (n) & 1,097 & - & 125 & - & 1,222 \\
\hline (2) & 367 & - & 48 & 2 & 416 \\
\hline Unknown & - & - & 12 & 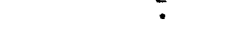 & 218 \\
\hline 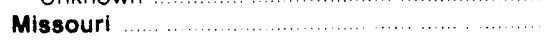 & 920 & - & 8 & 67 & 2997 \\
\hline Raslroad & 10 & - & - & - & 10 \\
\hline Truck & 89 & - & 8 & 67 & 164 \\
\hline Tramway. Conveyor, and Slurry Pipelıne ...... & 821 & - & - & - & 821 \\
\hline Unknown & - & - & - & - & 22 \\
\hline Montana $\ldots \ldots \ldots \ldots$ & 17,449 & - & 426 & 27 & 217,903 \\
\hline Railroad & 9,898 & - & 389 & 6 & 10,293 \\
\hline River & 102 & - & - & - & 102 \\
\hline Great Lakes & 2,900 & - & 18 & - & 2.918 \\
\hline Truck & 151 & - & 19 & 21 & 191 \\
\hline Tramway, Conveyor, and Slurry Pipeline & 4,397 & - & - & - & 4,397 \\
\hline Unknowri & - & - & $\cdot$ & - & 22 \\
\hline New Mexico & 9,806 & - & 378 & 4 & 10,188 \\
\hline 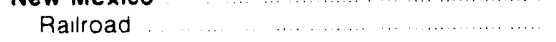 & 4.451 & - & 378 & ? & 4.829 \\
\hline$\ldots \ldots \ldots$ & - & - & - & 3 & 3 \\
\hline
\end{tabular}

See footnotes at end of table. 
Table 34. Domestic Distribution of U.S. Coal by Destination State, Consumer, Origin and Method of Transportation, January-June 1991 (Continued) (Thousand Short Tons)

\begin{tabular}{|c|c|c|c|c|c|}
\hline $\begin{array}{l}\text { Coal-Producing State of Origin } \\
\text { by Method of Transportation }\end{array}$ & $\begin{array}{l}\text { Electric } \\
\text { Utilities }\end{array}$ & $\begin{array}{l}\text { Coke } \\
\text { Plants }\end{array}$ & $\begin{array}{c}\text { Industilal } \\
\text { Plants } \\
\text { (Except Coke) }\end{array}$ & $\begin{array}{l}\text { Residerival } \\
\text { and commercial }\end{array}$ & Total \\
\hline & \multicolumn{5}{|c|}{ DESTINATION: U.S. TOTAL (Continued) } \\
\hline \multicolumn{6}{|l|}{ New Mexico } \\
\hline Tramway. Conveyor, and Slurry Pipeline ....... & 5,355 & - & - & - & 5.355 \\
\hline 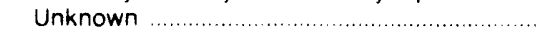 & - & - & - & * & $\cdot$ \\
\hline North Dakota & 11,566 & - & 2,969 & 70 & 14,604 \\
\hline Railroad & 2,191 & - & - & 12 & $\therefore 203$ \\
\hline 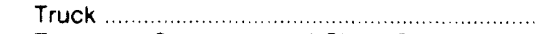 & 1,848 & - & 11 & 36 & 1,895 \\
\hline Tramway, Conveyor, and Slurry Pipeline ....... & 7,526 & - & 2.958 & 22 & 10,506 \\
\hline 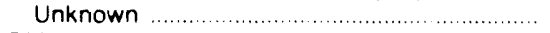 & - & - & - & 1 & 1 \\
\hline Ohio & 13,937 & - & 1,221 & 171 & 215,386 \\
\hline 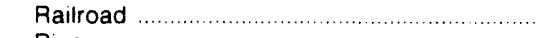 & 1,168 & - & 18 & 11 & 1.197 \\
\hline 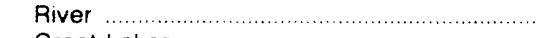 & 4,263 & - & 4 & - & 4.266 \\
\hline Great Lakes & 6 & - & 34 & $\cdot$ & 40 \\
\hline 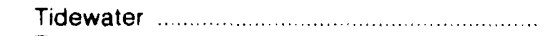 & 167 & - & - & - & 167 \\
\hline 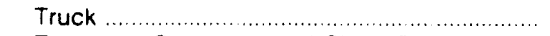 & 3,782 & - & 1,160 & 159 & 5.101 \\
\hline Tramway, Conveyor, and Slurry Pipeline ....... & 4.551 & - & - & - & 4.551 \\
\hline 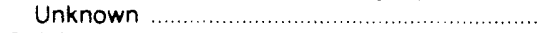 & - & - & 5 & $\cdot$ & 262 \\
\hline 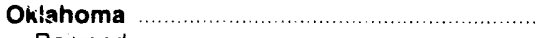 & 444 & - & 346 & • & 2793 \\
\hline Rairoad & 12 & - & 77 & - & 90 \\
\hline 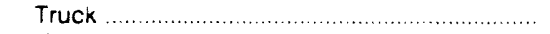 & 432 & - & 268 & $\cdot$ & 700 \\
\hline 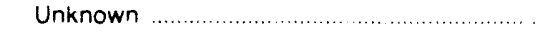 & - & - & - & • & 23 \\
\hline 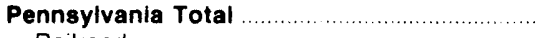 & 23,951 & 1,836 & 2,201 & 791 & 228,948 \\
\hline Railroad & 11.721 & 1,041 & 398 & 48 & 13,209 \\
\hline 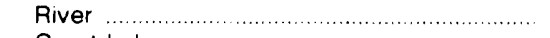 & 2,290 & 709 & 195 & - & 3,194 \\
\hline Great Lakes & 192 & 28 & 101 & - & 322 \\
\hline 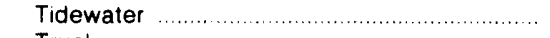 & 100 & - & - & - & 100 \\
\hline 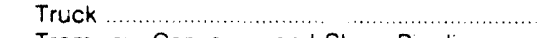 & 4.709 & 58 & 1,480 & 740 & 6,986 \\
\hline Tramway, Conveyor, and Slurry Pipeline ...... & 4,939 & - & - & - & 4,939 \\
\hline 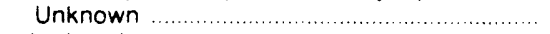 & - & - & 26 & 3 & 2198 \\
\hline 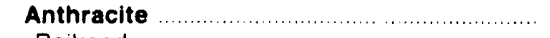 & 490 & * & 292 & 425 & 21,227 \\
\hline 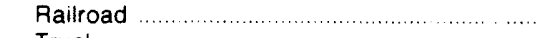 & - & * & 53 & 31 & 84 \\
\hline Truck & 296 & - & 239 & 392 & 926 \\
\hline Tramway, Conveyor. and Slurry Pipeline ....... & 194 & - & - & - & 194 \\
\hline Unknown & - & - & 1 & 2 & 223 \\
\hline Bituminous & 23,461 & 1,836 & 1,908 & 366 & 227,721 \\
\hline Railroad & 11,721 & 1.041 & 345 & 17 & 13.125 \\
\hline 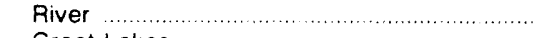 & 2.290 & 709 & 195 & - & 3.194 \\
\hline Great Lakes & 192 & 28 & 101 & - & 322 \\
\hline 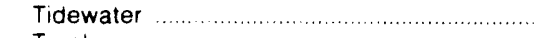 & 100 & - & - & - & 100 \\
\hline 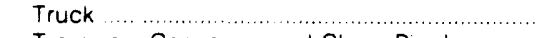 & 4.413 & 58 & 1,241 & 348 & 6.060 \\
\hline Tramway, Conveyor, and Slurry Pipeline ...... & 4,745 & - & - & - & 4.745 \\
\hline 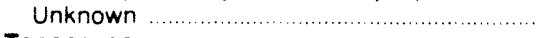 & - & - & 25 & 1 & 2175 \\
\hline Tennessee & 1,636 & - & 629 & 79 & 22,388 \\
\hline Railroad & 1,218 & - & 263 & 57 & 1.538 \\
\hline 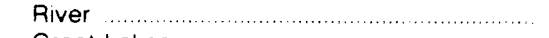 & 416 & - & 121 & 3 & 539 \\
\hline 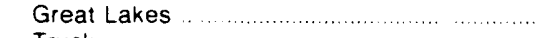 & $\bullet$ & - & 18 & - & 18 \\
\hline 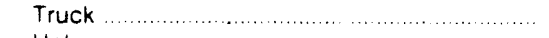 & 1 & - & 227 & 19 & 248 \\
\hline 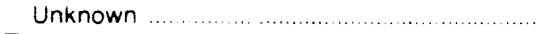 & - & - & - & $\cdot$ & 244 \\
\hline Texas & 24,941 & - & 917 & - & 25,858 \\
\hline 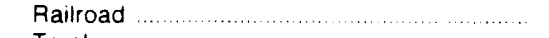 & 10,570 & - & 144 & - & 10.714 \\
\hline Truck & 6,403 & - & 118 & - & 6,521 \\
\hline Tramway. Conveyor, and Slurry Pipeline ....... & 7.968 & - & 655 & - & 8.623 \\
\hline Utah & 7,358 & 301 & 1.787 & 269 & 9,715 \\
\hline Railroad & 3.701 & 301 & 1.415 & 73 & 5.490 \\
\hline Truck & 2.463 & - & 371 & 194 & 3,028 \\
\hline Tramway, Conveyor, and Slurry Pipeline $\ldots$ & 1.194 & - & - & - & 1.194 \\
\hline 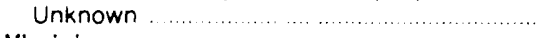 & - & - & 1 & 2 & 3 \\
\hline Virginia $\ldots \ldots \ldots$ & 7,079 & 3,044 & 2,058 & 46 & ${ }^{3} 12,252$ \\
\hline Rallroad $\ldots \ldots \ldots \ldots$ & 6.312 & 1,514 & 2.009 & 39 & 9.905 \\
\hline 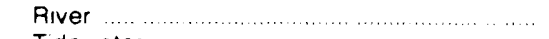 & 40 & 1.014 & - & - & 1.054 \\
\hline Tidewater & 696 & 36 & - & - & 733 \\
\hline 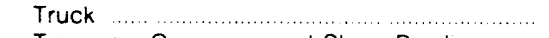 & - & - & 37 & 3 & 40 \\
\hline Tramway, Conveyor, and Slurry Pipeline ....... & - & 480 & - & - & 480 \\
\hline 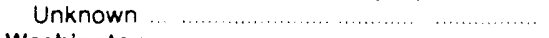 & - & - & 13 & 3 & ${ }^{3} 40$ \\
\hline 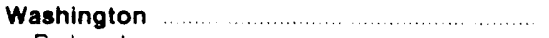 & 2,193 & - & - & * & 2,193 \\
\hline 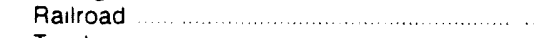 & 2.193 & - & - & - & 2,193 \\
\hline Truck ..... & $\cdot$ & - & - & $\cdot$ & . \\
\hline
\end{tabular}

See footnotes at end of table 
Table 34. Domestic Distribution of U.S. Coal by Destination State, Consumer, Origin and Method of Transportation, January-June 1991 (Continued) (Thousand Short Tons)

\begin{tabular}{|c|c|c|c|c|c|}
\hline $\begin{array}{l}\text { Coal-Producing State of Origin } \\
\text { by Method of Transportation }\end{array}$ & $\begin{array}{l}\text { Electric } \\
\text { Utilitios }\end{array}$ & $\begin{array}{l}\text { Coke } \\
\text { Plants }\end{array}$ & $\begin{array}{c}\text { Induatrial } \\
\text { Plante } \\
\text { (Except Coke) }\end{array}$ & $\begin{array}{l}\text { Realdential } \\
\text { and Commerclal }\end{array}$ & Total \\
\hline & \multicolumn{5}{|c|}{ DESTINATION: U.S. TOTAL (Continued) } \\
\hline West Virginla Total & 43,380 & 6,058 & 4,928 & 254 & ${ }^{3} 54,994$ \\
\hline Railroad & 22,836 & 4,680 & 2,976 & 55 & 30,547 \\
\hline 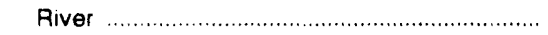 & 13,960 & 1,354 & 724 & 4 & 16,043 \\
\hline 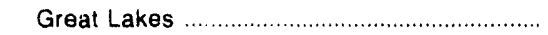 & 164 & 24 & 144 & 1 & 334 \\
\hline 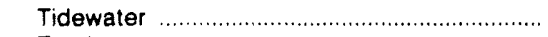 & 1,769 & - & 7 & - & 1,775 \\
\hline Truck & 2,369 & $\cdot$ & 1,027 & 188 & 3,583 \\
\hline Tramway, Conveyor, and Slurry Pipeline ....... & 2,282 & - & - & - & 2,282 \\
\hline Unknown & - & - & 51 & 7 & 3430 \\
\hline Northern & 20,795 & $\approx$ & 1,425 & 136 & 222,594 \\
\hline 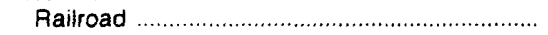 & 7,873 & - & 879 & - & 8,753 \\
\hline 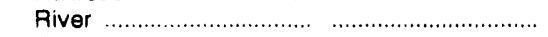 & 7,713 & - & 152 & - & 7,865 \\
\hline 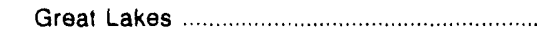 & 90 & - & 81 & - & 171 \\
\hline 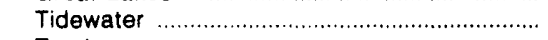 & 1,044 & - & - & - & 1,044 \\
\hline 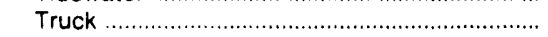 & 1,792 & - & 304 & 134 & 2,231 \\
\hline Tramway, Conveyor, and Slurry Pipeline ........ & 2,282 & - & - & - & 2,282 \\
\hline Unknown & & - & 9 & 1 & 2248 \\
\hline Southern & 22,586 & 6,058 & 3,504 & 119 & 332,400 \\
\hline 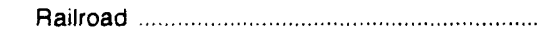 & 14,963 & 4,680 & 2,097 & 55 & 21,794 \\
\hline River & 6,247 & 1,354 & 573 & 4 & 8,178 \\
\hline Great Lakes & 75 & 24 & 63 & 1 & 163 \\
\hline 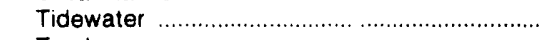 & 724 & - & 7 & - & 731 \\
\hline 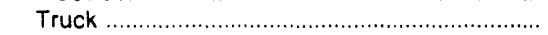 & 577 & - & 723 & 53 & 1,353 \\
\hline 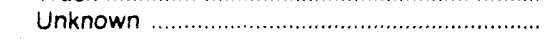 & - & - & 42 & 6 & 3182 \\
\hline 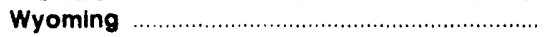 & 92,743 & - & 2,195 & 126 & 295,073 \\
\hline 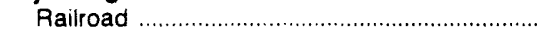 & 79,917 & - & 1,840 & 65 & 81,829 \\
\hline River & 6,796 & - & - & - & 6.796 \\
\hline Great Lakes & 233 & - & - & - & 233 \\
\hline Truck & 1,009 & - & 339 & 60 & 1,408 \\
\hline Tramway, Conveyor, and Slurry Pipeline ....... & 4,788 & - & - & - & 4,788 \\
\hline 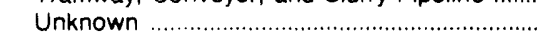 & - & - & 9 & 1 & 220 \\
\hline U.S. Total & 379,800 & 14,272 & 33,374 & 3,173 & 3432,457 \\
\hline Railroad & 226,421 & 9,220 & 17,088 & 867 & 253,596 \\
\hline River …........... & 57,558 & 3,921 & 2.171 & 192 & 63,841 \\
\hline 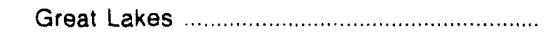 & 3,858 & 53 & 822 & 3 & 4,736 \\
\hline 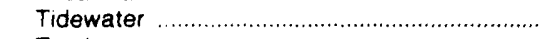 & 3,974 & 36 & 217 & - & 4,228 \\
\hline Truck & 36,762 & 563 & 9,256 & 2,032 & 48,613 \\
\hline Tramway, Conveyor, and Slurry Pipeline ........ & 51,227 & 480 & 3,613 & 22 & 55,342 \\
\hline 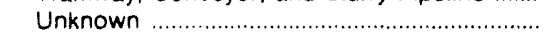 & - & - & 207 & 57 & 32,103 \\
\hline
\end{tabular}

1 Includes distribution to the transportation sector.

2 includes distribution to unknown consumers.

3 Includes distribution to both the transportation sector and unknown consumers.

- Quantity is less than 500 short tons or percent is less than 05 .

Note: Total may not equal sum of components because of independent rounding.

Source: Energy Information Administration, Form ElA-6, "Coal Distribution Report." 


\section{Appendix A}

\section{Explanatory Notes}

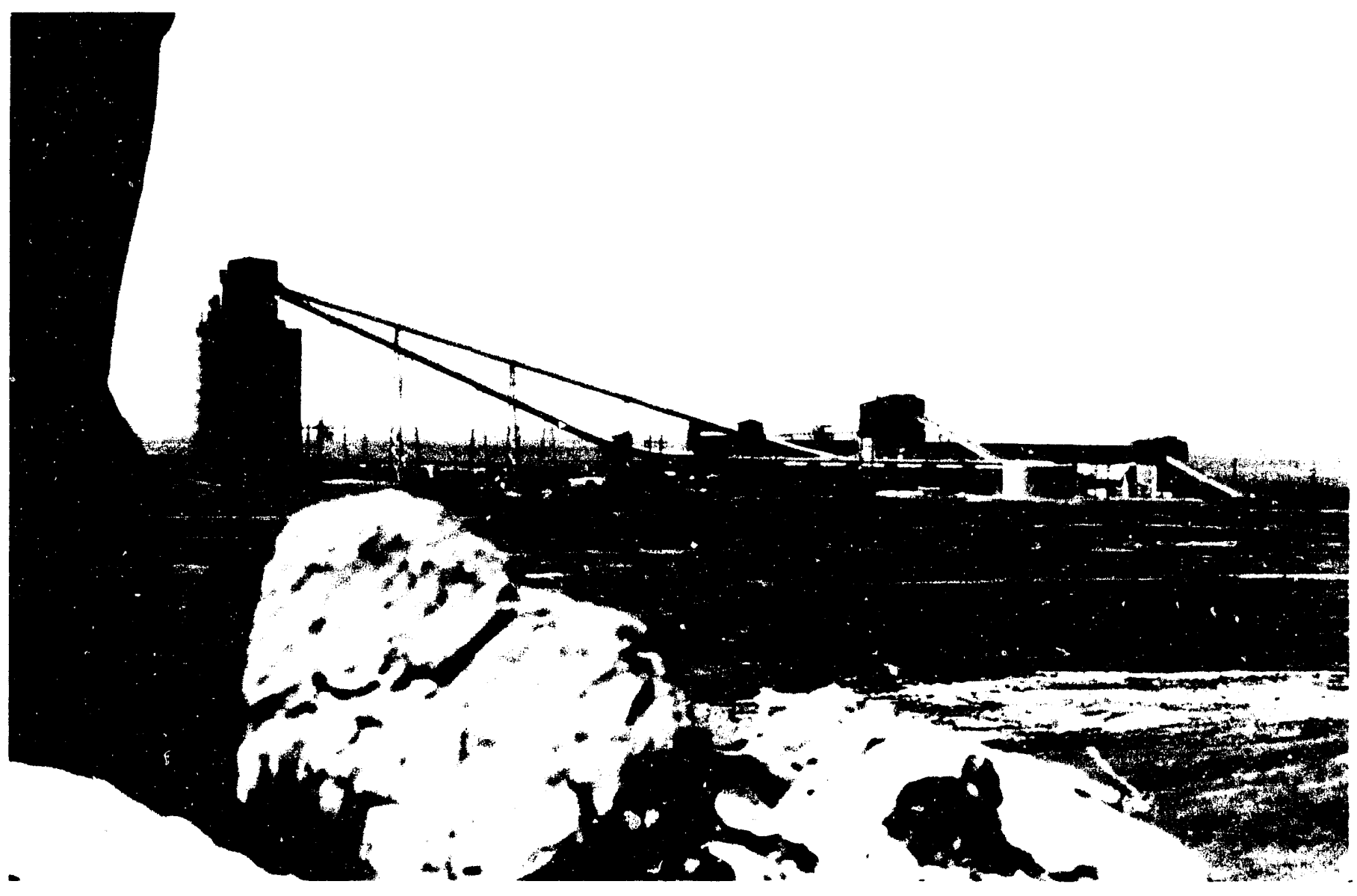

The storage facility at the Thunder Basin Coal Company's Black Thunder mine in Wyoming. This mine is the Vation's singie iurgest prociucer. 


\section{Appendix A}

\section{Explanatory Notes}

\section{Sources of Data}

\begin{abstract}
All data in this report were collected on Form EIA-6 "Coal Distribution Report," by the Energy Information Administration (EIA), U.S. Department of Energy. The data contain information on coal production and purchases, coal stocks, and coal distribution by consumer category, by method of transportation, by coal-producing State of origin, and by State of destination.
\end{abstract}

\section{Coverage}

The data presented in this report were submitted--as required by law--by companies (coal-mining companies, wholesale coal dealers, and brokers) that owned or purchased and distributed in excess of 50,000 short tons of coa! annually. This report also includes data that were voluntarily submitted by other coal distributo:s who are under the reporting threshold. Currently, there are approximately 1,300 respondents.

In this publication, "United States" refers to the 50 States and the District of Columbia. No coal is produced in Hawaii, and none of the coal produced in the continental United States is shipped to Hawaii.

\section{Respondent Mailing List}

The respondent list for this survey is updated by comparison with lists of coal producers from the Mine Safety and Health Administration, U.S. Department of Labor, and to similar lists maintained by various State agencies. Also, new respondents are frequently identified on Form EIA-6 itself when other companies are named as sources of coal purchases.

\section{Adjustments}

No estimates or other source data are included in this report. Revisions are not made to quarterly data for prior quarters in a year until the fourth quarter, when all data are finalized. No data are revised unless a significant error is found (i.e. an error of more than 5 percent at the national or State levels).

Annual data (i.e., January through December) published in April of the following year are final, and no changes are made in those data as a result of first quarter reports in the following year. When comparing final data to preliminary data, revision errors are less than 1 percent at national total levels. The nonsampling error for this survey is less than 1 percent, measured against national totals.

\section{Technical Notes}

\section{Coal Production and Purchases}

The amount of coal produced is reported by Form EIA-6 respondents as coal production and/or purchases.

State of Origin: The State of Origin is the State where the coal was mined or produced, and is not influenced by transhipments.

Production: When a responding company produces and distributes its own coal to a consumer, the coal is reported as production.

Purchases: When a responding company purchases coal produced by another company, the coal is reported as a purchase. This coal may be purchased from current production or stocks. Companies purchasing coal are frequently wholesale coal dealers (including brokers) or retail coal dealers. 
For a reported purchase of coal from another Form EIA-6 respondent, the tonnage is included in the report of the purchaser and deleted from the report of the seller, eliminating the duplicate tonnage.

\section{Coal Stocks}

Coal stocks consist of the supply of coal held in stock by producers/distributors at the end of a reporting quarter.

\section{Consumer Categories}

Electric Utilities: All privately owned companies and all publicly owned agencies engaged in the generation, transmission, or distribution of electric power for public use. Publicly owned agencies include municipal electric utilities; Federal power projects, such as the Tennessee Valley Authority (T.V.A.); and rural electrification cooperatives, power districts, and State power projects.

Coke Plants: All plants where coal is carbonized for the manufacture of coke in slot or beehive ovens. Only coal that is carbonized to produce coke is reported. Shipments of coal to coke plants for use in space heating or electric power generation and coal used to produce formcoke are included in the other industrial consumer category.

Other Industrial: Industrial users, not including coke plants, that are engaged in the mechanical or chemical transformation of materials or substances into new products (manufacturing); and companies engaged in agriculture, mining (othcr than coal mining), or construction industries. This category also includes coal used for gasification or liquefaction, and coal used at the respondents' mines.

Residential and Commercial: Housing units; wholesale and retail businesses (except coal wholesale dealers); health institutions (hospitals); social and educational institutions (schools and universities); and Federal, State, and local governments (military installations, prisons, office buildings). Coal sold by respondents to their employees is included in this category.

Transportation: Shipments of coal to railroad companies to be used as fuel and shipments of coal to be used as vessel/ship fuel.

\section{Methods of Transportation}

Rail: Shipments of coal moved to consumers by rail, either private or public/commercial. Included is coal hauled to or away from a railroad siding by truck.

Water Transportation: Shipments of coal moved by one of three methods--river, great lakes, or tidewater piers and coastal ports. Included in these shipments is coal hauled to or from water-loading facilities by other means of transportation.

River: Shipments of coal moved to consumers via river by barge, except shipments to Great Lakes coalloading docks or tidewater piers or coastal ports.

Great Lakes: Shipments of coal moved to consumers via the Great Lakes. These shipments are moved via the Great Lakes coal-loading docks, which are identified by name and location as follows: Superior Midwest Energy Terminal, Superior, Wisconsin; Bessemer \& Lake Erie Coal Storage \& Transfer Facility, Conneaut, Ohio; B\&O Railroad Coal-Loading Dock, Lorain, Ohio; C\&O Railroad Presque Isle Docks, Toledo, Ohio; Lakefront Dock \& Railroad Terminal Company Coal-Loading Dock, Toledo, Ohio; N\&W Sandusky Coal Pier No. 3, Sandusky, Ohio; ConRail Coal Transfer Facilities, Ashtabula, Ohio; Rail to Water Transfer Corporation Dock, Chicago, Illinois.

Tidewater Piers and Coastal Ports: Shipments of coal moved to tidewater piers and coastal ports for further shipments to consumers via coastal water or ocean. The tidewater piers are identified by name and location as follows: B\&O Curtis Bay Coal Piers, Baltimore, Maryland; C\&O Coal Piers Nos. 14 \& 15, Newport News, Virginia; N\&W Lamberts Point Coal Piers Nos. $5 \&$ 6, Norfolk, Virginia; Alabama State Docks Bulk Handling Plant, Mobile, Alabama; Alabama State Docks/McDuffie Terminals, Mobile, Alabarna; Canton Coa' Pier, Baltimore Harbor on Chesapeake Bay; Greenwich Coal Pier, Greenwich Point, Philadelphia, $\mathrm{Pa}$. on Delaware River; Port Richmond Pier, Pier 18-Port Richmond, Philadelphia, $\mathrm{Pa}$. on Delaware River; Galveston Regional Coal Distribution Center, Pelican Island, Galveston, Texas; International Marine Terminals/Plaquemines Parish Terminal, Mile 57 AHP-Mississippi River, approx. 30 miles south of New Orleans; Energy Terminals of Houston, Inc., a Subsidiary of Soros Associates, Houston, Texas. Coastal Ports are those located at Charleston, South Carolina; New York, New York; San Diego, California; Los Angeles, California; and Seattle, Washington.

Truck: Shipments of coal moved from producers to consumers by truck

Tramway, Conveyor, or Slurry Pipeline: Shipments of coal moved to consumers by tramway, conveyor, or slurry pipeline. 


\title{
Appendix B
}

\author{
Form EIA-6 and \\ Instructions
}

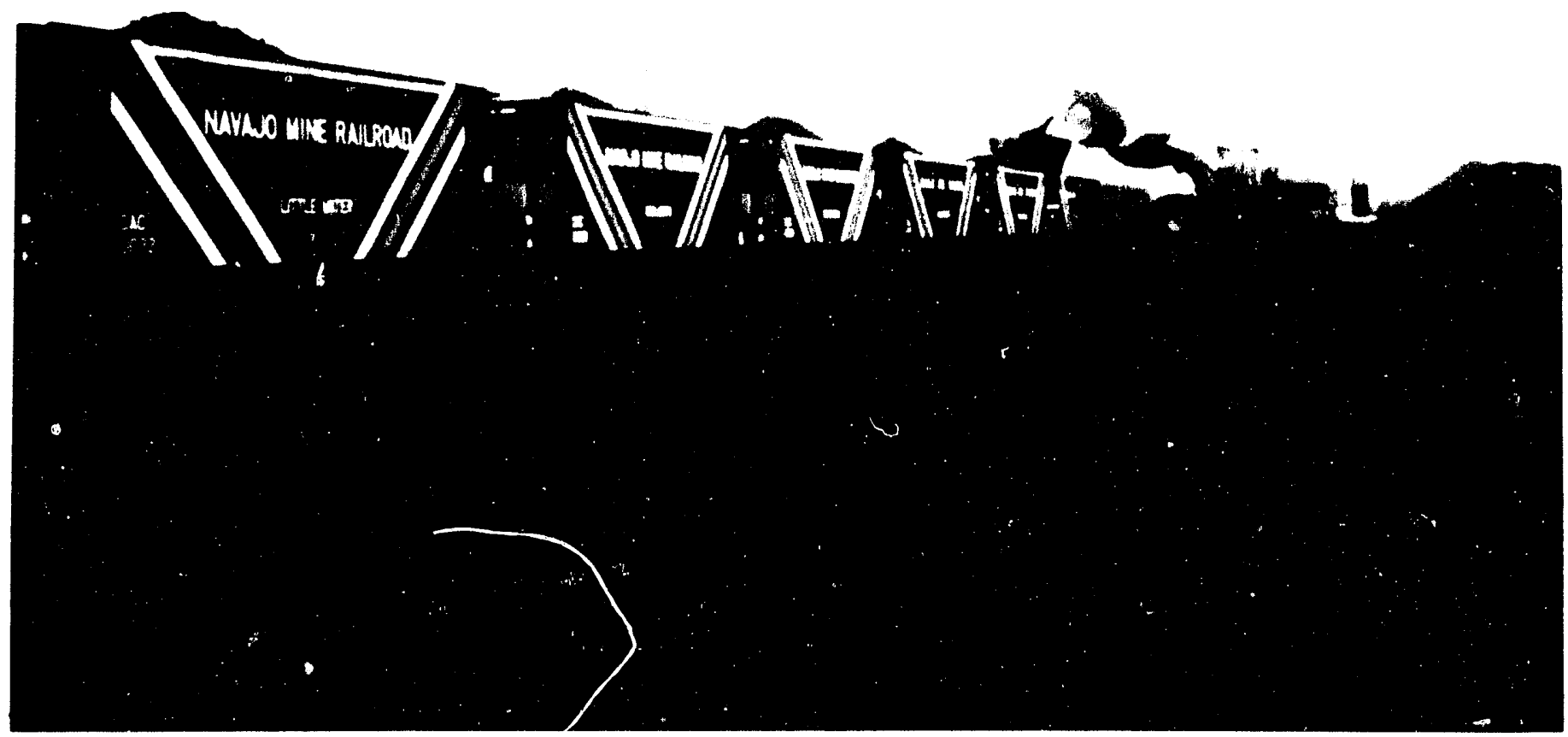

Coal from the Navajo mine near Fruilland. New. Mexico. being loaded on rail cars for transit to the nearby Four Corners power plant. 
Coal Distribution Report

EIA-6 (January 1990)

This repon is mandalory under Pubtc: aw 93.275 Falure 10 comply may result in criminal tines. covil penalties and other sanctions as provided by law For the provisions concerning ine

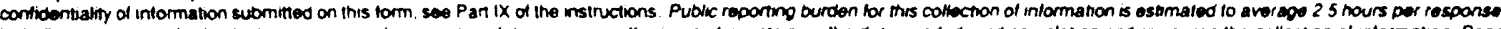
including the time reviowing instuctions, searching existing data sources. gathering and mantaining the data neded. and completing and roviewning the collection of intormation Send

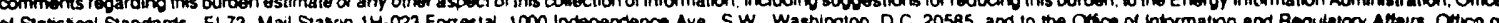
of Statsocal Slandats. Ek.7. Manepernent and Budpet, Washington, D.C 20503

I. RESPONDENT IDENTIFICATION

Please read the Instructions belore completing this form

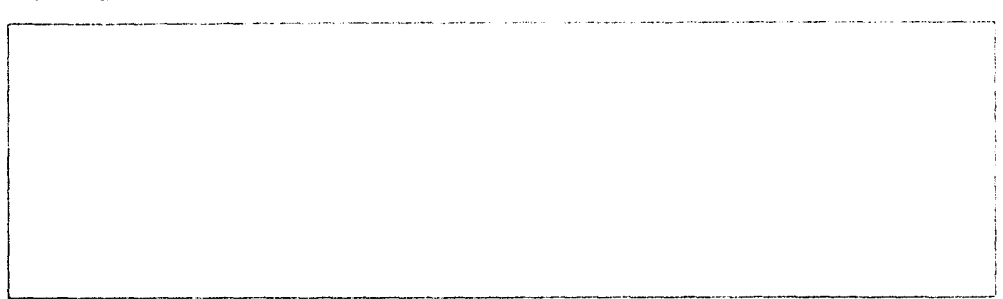

It the above name and adoress label is incorrect, phease make changes in the space provided at the noth.

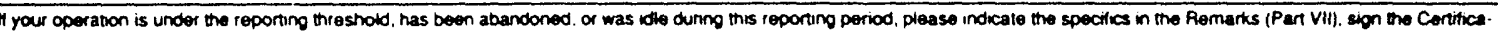

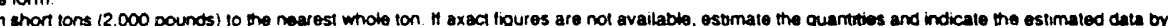
decing (est.) in front of the reported data inem.

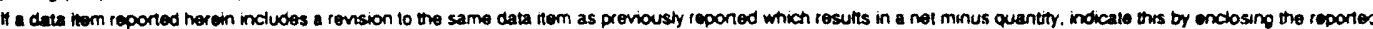
aumitity in parentroses ( )

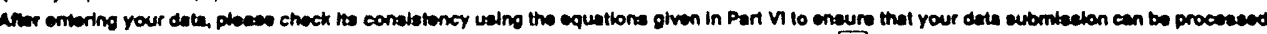

If you desire a tree subscription to the publication, "Coa Dstritubon" quanterty, ploase check nere.

A separate Form EIA-6 must be submitted for each coal-producing State in which coal is minod.

(See Part $X \alpha$ ithe instructions lor a hist of States.)

a. Y Your Compeny Chenger Ownerahlp Ouring the Ouarter, enter the dete of chenge:

and, as applicable, the name and the address of new owner

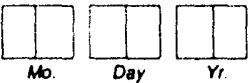

Name of Company

Polnt of Contact (Name \& Title)

Area Code \& Office i eiepriune

3. Neme(s) and Addroea(ce) of Parent Compeny(les):

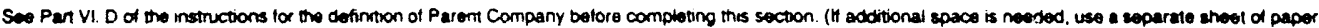

\begin{tabular}{|c|c|c|}
\hline $\begin{array}{l}\text { Name of Parent Company } \\
\text { (I) dimenent from reaponding compeny) }\end{array}$ & $\begin{array}{l}\text { Address } \\
\text { (Street, Clty, State, Zlp) }\end{array}$ & $\begin{array}{l}\text { Polnt of Contact } \\
\text { (Neme, Telephone No.) }\end{array}$ \\
\hline & & \\
\hline & & \\
\hline
\end{tabular}

C. Coetproducting Stat

Enter the name of the cosal-producing State of ongin that you are reporting data for in this repon (See Part $X$ of the instructions for a list of Stales) State

II. ORIGIN OF COAL

A. Coal Produced at Your Company's Mines during the Quarter:

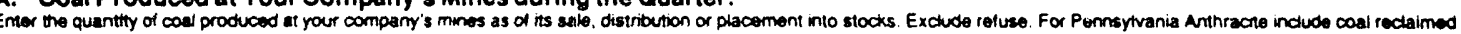

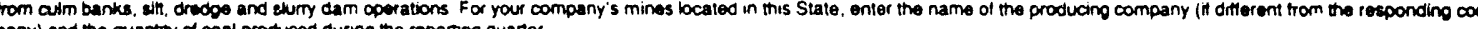
pany) and the quantry $\alpha$ cod produced during the reporteng quarter

\begin{tabular}{l|l}
\hline $\begin{array}{l}\text { Name of Producing Company } \\
\text { (t different from responding compeny) }\end{array}$ & $\begin{array}{l}\text { Quantity Produced } \\
\text { (Shon ions) }\end{array}$ \\
\hline & \\
\hline & \\
\hline Total Coal Produced at Your Company's Mines in this State during the Quarter, excluding refuse. & \\
\hline
\end{tabular}

B. Coal Purchased from Other Companies during the Quarter which was Produced in this State:

Repont here the quantify of coal purchased trom other companes dunno the reporning quarter which was produced in this coal-producing State. For bach company. enter the company name and address and the quantity of coal purctiased (th additional space is nopded. use a separate sheet of paper)

\begin{tabular}{l|l|l}
\hline Name of Company & $\begin{array}{l}\text { Address } \\
\text { (Stret. City, State. Zup Code) }\end{array}$ & $\begin{array}{l}\text { Quantity Purchased } \\
\text { (Shorn Tons) }\end{array}$ \\
\hline & & \\
\hline & & \\
\hline Total Coal Purchased from Other Companies Ciuring the Quarter & \\
which was Produced in this State, excluding reluse. & \\
\hline C. Total Coal Produced and/or Purchased during the Quarter whlch \\
Was Mined in this State, excluding refuse. \\
(Sum of totas in Parts $A$ and 8 )
\end{tabular}


Energy Information Administration

Coal Distribution Report

EIA-6 (January 1990) Page 2

\section{DISTRIBUTION FOR WHICH THE CONS, IMEA CATEGORY IS KNOWN}

Repont here the distribution during the reporting quarter of that por bo of the coal reponed in Parl II C, plus any coal distributed trom stocks located in this coal.producing Stale tor which you know the consumer. Pleme read parts VI. A. B. and C of the if structions betore comploting Pan ill so that the consumer categories. methods of transportation

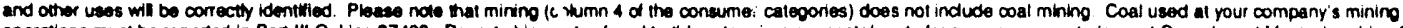
opertions must be reported in Pan III. G. Une 37499. Report sthiprnet 's of coal to wdewater piers or coastal ports lor over seas exports (except Canada and Mexico) on Line 34802

A. Shipments by Rall

\begin{tabular}{|c|c|c|c|c|c|c|c|}
\hline \multirow[t]{2}{*}{ Destination } & \multicolumn{4}{|c|}{ Quantity Distributed by Conau ner Categories (Short Tons) } & \multirow[b]{2}{*}{$\begin{array}{l}\text { Residential/ } \\
\text { Commercial } \\
\text { (5) }\end{array}$} & \multirow[b]{2}{*}{$\begin{array}{l}\text { Total } \\
\text { (6) }\end{array}$} & \multirow[b]{3}{*}{31001} \\
\hline & $\begin{array}{l}\text { Electric } \\
\text { Utilities } \\
\text { (1) }\end{array}$ & $\begin{array}{l}\text { Coke Plants } \\
\text { (2) }\end{array}$ & $\begin{array}{l}\text { Manufacturing } \\
\text { (except Coke } \\
\text { Plants) } \\
\text { (3) }\end{array}$ & $\begin{array}{l}\text { Agriculture, } \\
\text { Mining, and } \\
\text { Construction } \\
\text { (4) }\end{array}$ & & & \\
\hline $\begin{array}{l}\text { U.S. } \\
\text { Alabama }\end{array}$ & & & & & & & \\
\hline \multicolumn{7}{|l|}{ Alaska } & 31002 \\
\hline \multicolumn{7}{|l|}{ Arizona } & 31004 \\
\hline \multicolumn{7}{|l|}{ Arkansas } & 31005 \\
\hline \multicolumn{7}{|l|}{ California } & 31006 \\
\hline \multicolumn{7}{|l|}{ Colorado } & 31008 \\
\hline \multicolumn{7}{|l|}{ Connecticut } & 31009 \\
\hline \multicolumn{7}{|l|}{ Delaware } & 31010 \\
\hline \multicolumn{7}{|l|}{ District of Columbia } & 31011 \\
\hline \multicolumn{7}{|l|}{ Florida } & 31012 \\
\hline \multicolumn{7}{|l|}{ Georgia } & 31013 \\
\hline \multicolumn{7}{|l|}{ Idaho } & 31016 \\
\hline \multicolumn{7}{|l|}{ Illinois } & 31017 \\
\hline \multicolumn{7}{|l|}{ Indiana } & 31018 \\
\hline lowa & & & & & & & 31018 \\
\hline Kansas & & & & & & & 31020 \\
\hline Kentucky & & & & & & & 31021 \\
\hline Louisiana & & & & & & & 31022 \\
\hline Maine & & & & & & & 31023 \\
\hline Maryland & & & & & & & 31024 \\
\hline Massachusetts & & & & & & & 31025 \\
\hline Michigan & & & & & & & 31026 \\
\hline Minnesota & & & & & & & 31027 \\
\hline Mississippi & & & & & & & 31028 \\
\hline Missouri & & & & & & & 31029 \\
\hline Montana & & & & & & & 31030 \\
\hline Nebraska & & & & & & & 31031 \\
\hline Nevada & & & & & & & 31032 \\
\hline New Hampshire & & & & & & & 31033 \\
\hline Now Jersey & & & & & & & 31034 \\
\hline New Mexico & & & & & & & 31035 \\
\hline New York & & & & & & & 31036 \\
\hline North Carolina & & & & & & & 31037 \\
\hline North Dakota & & & & & & & 31038 \\
\hline Ohio & & & & & & & 31039 \\
\hline Oklahoma & & & & & & & 31040 \\
\hline Oregon & & & & & & & 31041 \\
\hline Pennsylvania & & & & & & & 31042 \\
\hline Rhode Island & & & & & & & 31044 \\
\hline South Carolina & & & & & & & 31045 \\
\hline South Dakota & & & & & & & 31046 \\
\hline Tennessee & & & & & & & 31047 \\
\hline Texas & & & & & & & 31048 \\
\hline Utah & & & & & & & 31049 \\
\hline Vermont & & & & & & & 31050 \\
\hline Virginia & & & & & & & 31051 \\
\hline Washington & & & & & & & 31053 \\
\hline West Virginia & & & & & & & 31054 \\
\hline Wisconsin & & & & & & & 31055 \\
\hline Wyoming & & & & & & & 31056 \\
\hline Canada & & & & & & & 31122 \\
\hline Mexiro & & & & & & & 31201 \\
\hline Total Rall Shlpments & & & & & & & 31199 \\
\hline
\end{tabular}


Energy Information Administration Coal Distribution Report

EIA-6 (January 1990) Page 3

\section{U.S. Department of Energy}

i.D. No. $0[0[0] 9[9] 01 \mathrm{~L}]$

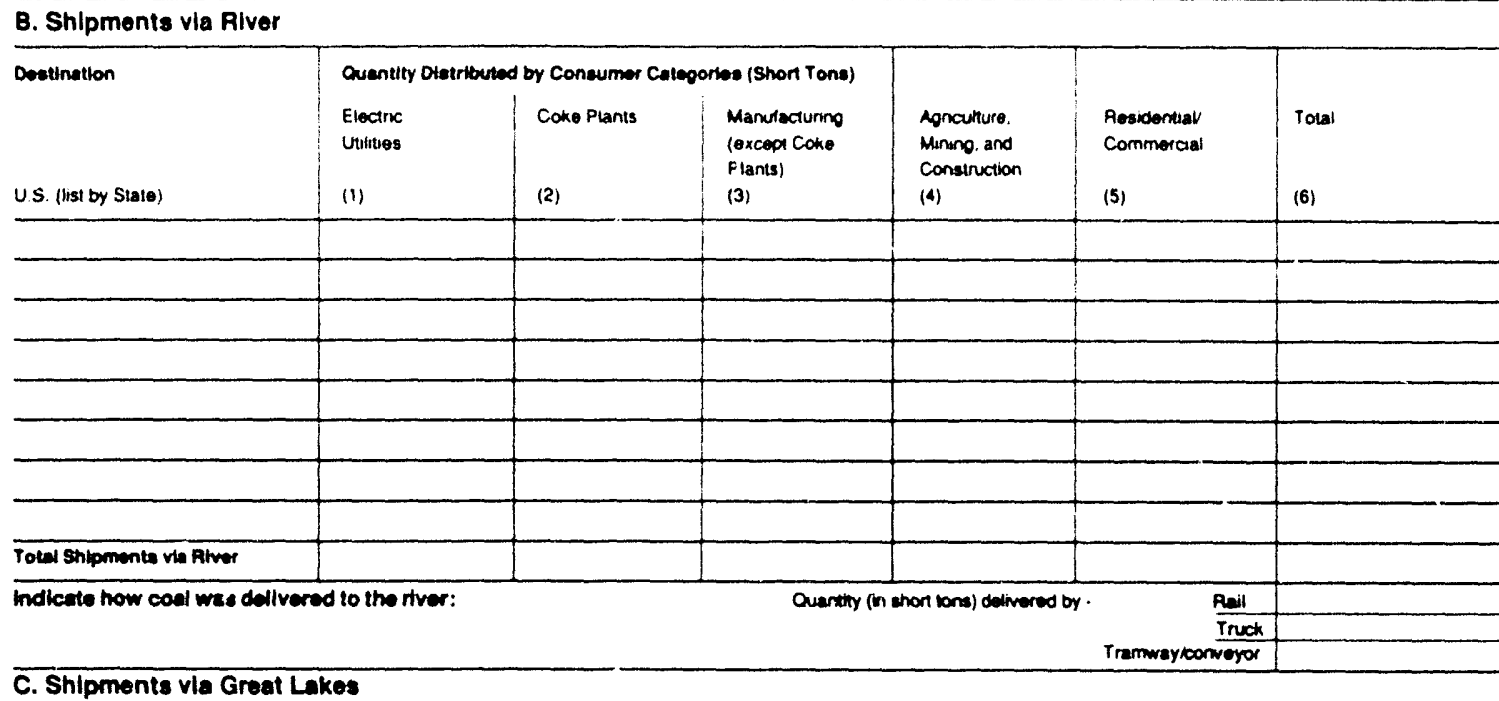

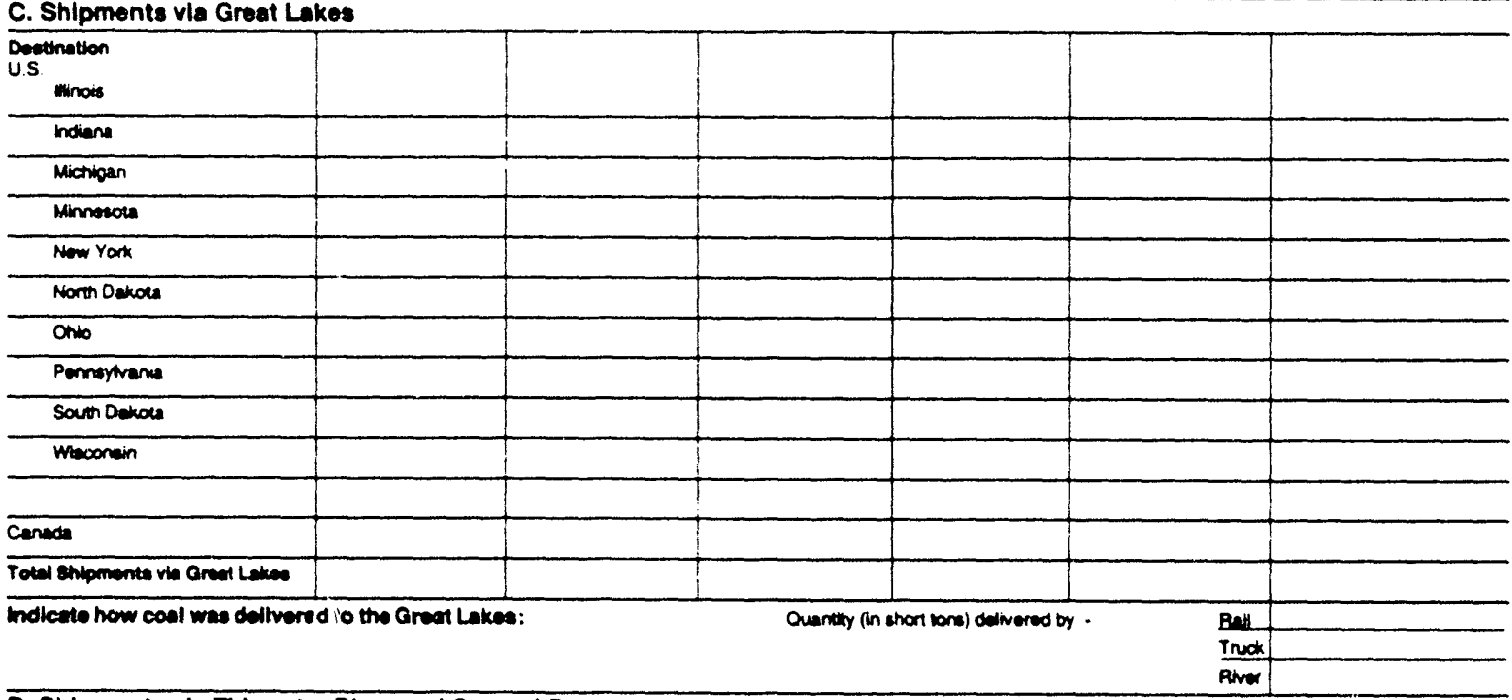

D. Shlpments via Tidowater Plors and Coestal Ports 
Energy Information Administration

Coal Distribution Report

U.S. Department of Energy

EIA-6 (January 1990) Page 4

E. Shlpments via Truck

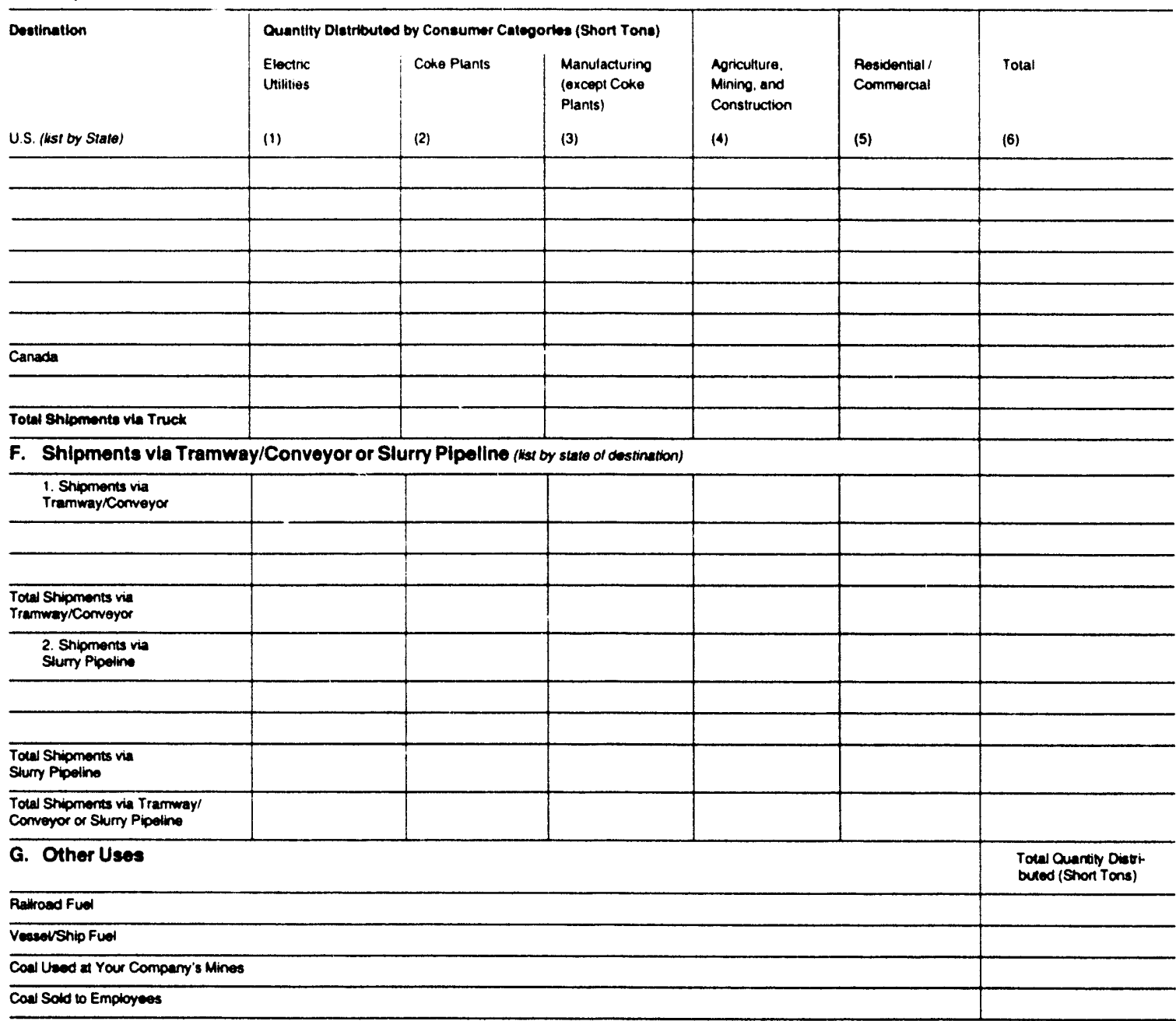

H. Sales of Coal to Other Coal Distribution Companles For Which You Know and Reported the Consumer Category or Overseas Exports.

Enter betow the name(s) and addrese(es) of coal distribution compenies who purchased any of your coal that was reponted in Pent III A-G.

For each company. idenaty the quentihy of coal purchased from you during the reporting quenter and indicale where the coal was reportid in Part III of the torm. (If edditionial space in noeded, use a separate shoel of peaper.)

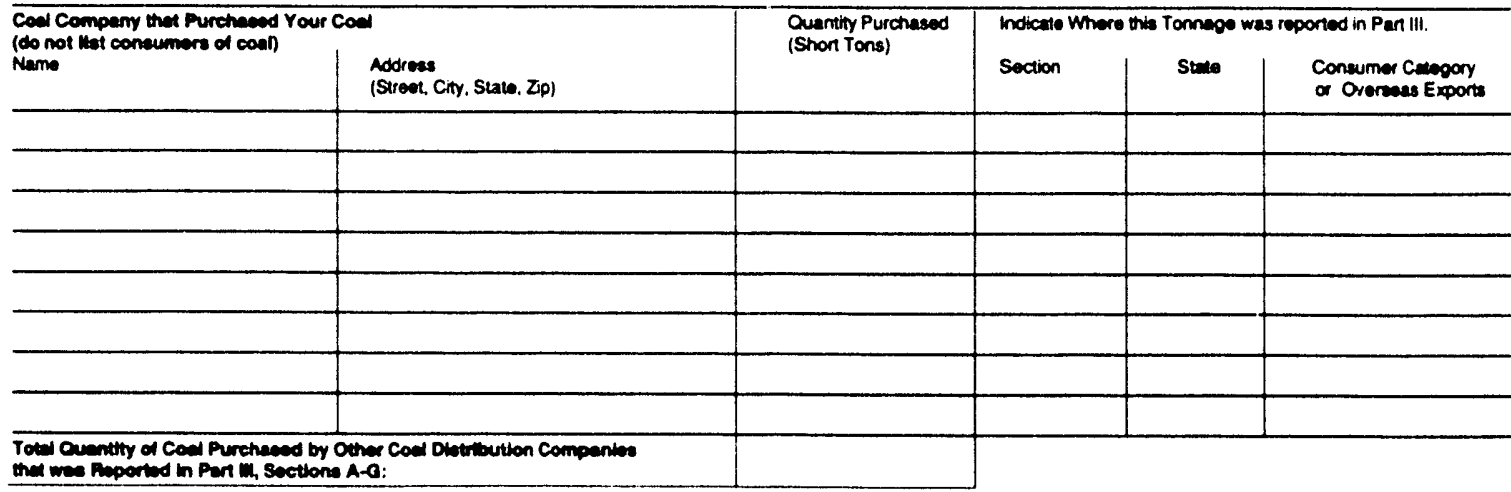


Energy Information Administration

Coal Distribution Report

EIA-6 (January 1990) Page 5

\section{U.S. Department of Energy}$$
\text { 1.0. No. }
$$

\section{DISTRIBUTION FOR WHICH THE CONSUMER CATEGORY IS UNKNOWN}

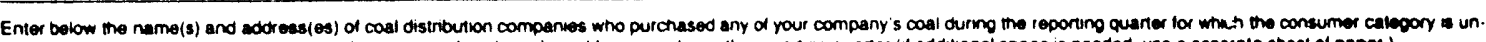
known to you. For each company. dentity the quantity of coal purchased trom you during the reporting quarter (it additional space is needed. use a separale sheel of paper) Report coal exported overseas in Pan ill D on ine 34802

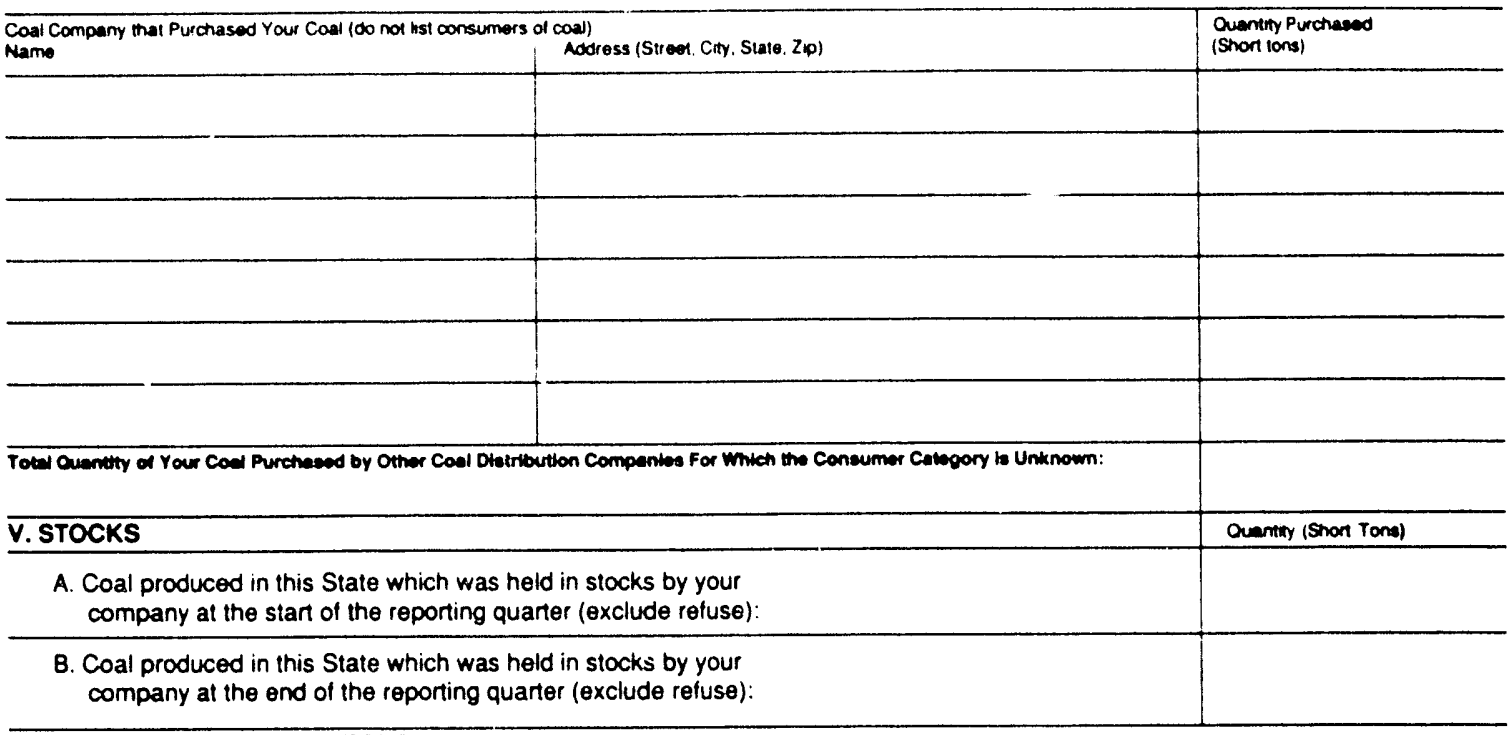

VI. DATA CONSISTENCY CHECKS

Tho foltowing equations should be uend to check the constatency of your data:

A stock Equation: Line code 51999 (curremt quarier) = Line code 52999 (prenous quaner)

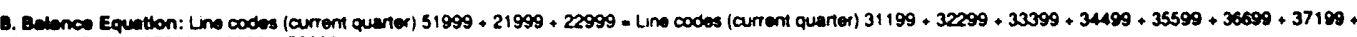
$37299+37499+37599+40999+52999$

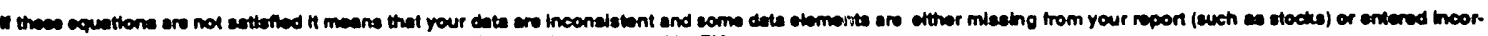
rectly. Theee equation must be setiefled before your date can be procesesed by EIA.

VII. REMARKS

\section{POINT OF CONTACT}

Emer the name. thite, and telephone number of your company representative who can answer questions regarding the information p. anded on this form. Name

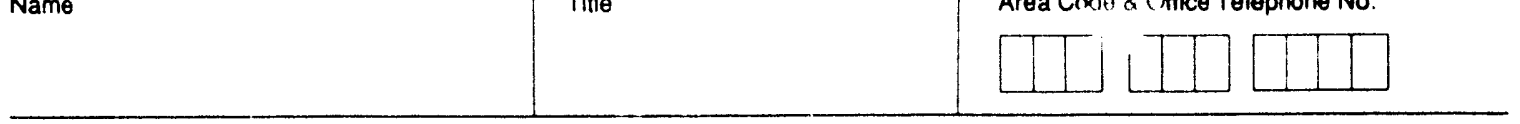

\section{CERTIFICATION}

Thes part of the lorm must be complened by the official of the reporting company empowered to centity the tivith and accuracy of the intormation provided on this lorm

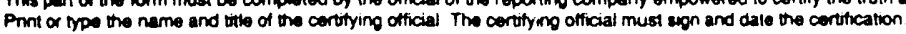

I certity that the information provided herein and appended hereto is true and accurate to the best of my knowledge.

Name
Title 18, U.S.C. $\$ 1001$ makes it a crime for any person knowingly and willingly to make to any Agency or Department of the
United States any false, fictitious or fraudulent statements or misrepresentation as to any matter within its jurisdiction.




\section{Furpose}

This survey collects coal distribution data from U S coal mining companies and wholesale and retait coal dealers, including brokers. Intormation on coal production and purchases, distribution and stocks is collected to provide Congress with basic statistics concerning the origin. method of transportation, consumer use, and destination of U.S coal. as iequired by the Federal Energy Administration ACt of 1974 (P.L 93-275) as amended

These data appear in the Coal Distribution Report, the Quarterly Coal Report, the Monthly Energy Review, and the State Energy Data Rejort. In addition, Ihe Energy Inlormation Administration uses the data lor coal transportation related analyses and in short and mid-ierm models (i.e. the Short. Term Coal Analusis System (SCOAL), the Shont. Term Integrated Forecasting System (STIFS), the Intermediate Future Forecasting System (IFFS), and the Coal Supply and Transportation Mojel (CSTM)). The forecast data appeat in the Short. Term Energy Outlook and the Annual Energy Outlook

II. Who Shall Submit

All companies in the 50 United States and the District of Columbia that owned or purchased and distributed in excess of 50,000 tons of coal during the year defined by the current reporting quarter and the previous three reporting quarters. These companies include coal mining companies, wholesale coal dealers (isiciuding brokers), and retall coal dealers. Companies that take custody (physical possession) of the coal and transport but never own the coal need not report. Retain the copy maiked "File Copy" for your records. A separate Form EIA.6 must be submitted for each coal-producing State in which coal originated. (See Pan $\mathrm{X}$ below).

III. Where to Submit

Combanies shall return completed EIA-6 form, to

Energy Information Administration, El-521

Mall Station: BG-094 Forst

U.S. Department of Energy

Washington, D.C. 20585

Attn: EIA-6

Requests for further intormation, additional forms and instructions. or an extension of the reporting deadine may be directed to the address above or by telephone to (202) $254-5400$.

IV. When to Submit

Form ElA-6 shall be submitted no later than one month after the close of each reporting quarter. The reporting quarters are January 1 - March 31 , April 1 - June 30 , July 1 . September 30. and October 1. December 31. The respective due dates are May 1. August 1, November 1, and February 1

\section{Sanctions}

The timely submission of Forn EIA-6 by a company required to report is a mandatory requirement. Late filing. failure to file, or failure to otherwise comply with these instructions may result in criminal fines. civil penalties and other sanctions provided by Section 13(i) of the FEA Act of 1974 (P.L. 93-275).

\section{Definitions}

\section{A. Consumer Categories}

1. Electric Uillities: All privately owned companies and all publicly owned agencies engaged in the production of electric power for public use. Publict, owned agencies include the following: Municipal electnc utlities: Federal power projects, such as the Tennessee Valley Authority (TVA); and rural electrification cooperatives, power distncts. and State power projocts

2. Coke Plents: All plants where coal is carbonized for the manufacture of coke in slot or beehive ovens. Report only coal that is carbonized to produce coke. Shipments of cosl to coke plants for use in space heating or electric power generation silould be included with "Manufacturing (except Coke Plants)." Do not report coal used to produce torm coke. to coke plants for use in space heating or electric power generation silould

include coal used for form coke with "Manulacturing (except Coke Plants). substances into new (1. $\theta$. finished or semifinished) products. Include coal used for gasificationMiquetaction.

4. Agriculture, Mining, and Construction: Companies engaged in agriculture, mining (other than coal mining), or construction industries.

5. Residential/Commercisl: Housing units; wholesale or retail businesses (except coal wholesale dealers); health institutions (hospitals); social and educational instrtutions

(schools and universities): and Federal. State, and local oovernments (miltary installations, prisons, office buildings, elc.). Exclude shioments to Federal power propects, such as

TVA: and rural electnfication cooperatives, power distrits, and State power projects. These are to be included in consumer category 1 (Electric Utilities).

\section{B. Methods of Transportation to Consumers}

1. Rall: Shipments of coal moved to consumers by rail (private or public/commercial). Included is coal hauled to or away from a railroad siding by truck.
2. River: Shipments of coal moved to consumers va river by barge Not inctuded are shipments to Great Lakes coal loading docks or tidewater piers or coastal ports.

2. Alver: Shipments of coal moved to consumers va river by barge Not inctuded are shipments to Great Lakes coal loading docks or tidewater piers or coastal ports.
3. Great Lekes: Shipments of roal moved to consumers via the Great Lakes. These shipments are moved via the Great Lakes coal loading docks, which are identified by

3. Great Lokes: Shipments

Supenor Midwest Energy Terminal, Superior, Wisconsin

Bessemer \&ake Erie Coal Storage and Transter Facility, Conneaut, Ohio

B\&O Railroad Coal Loading Dock, Lorain. Ohio

C8O Railroad Presque iste Docks. Toledo, Ohio

Laketront Dock and Railroad Terminal Company, Coal Loading Dock. Toledo, Onio

N\&W Sandusky Coal Pier No. 3, Sandusky. Ohio

ConRail Coa! Transter Facilities. Ashtabula, Onio

Rail to Water Transter Com. Dock, Chicago, Illinois

4. Thewater Piers and Cosstal Porte: Shipments of coal moved to Tidewater Piers and Coastal Ports for further shipments to consumers via coastal water or ocean The Tidewater

Piers are identified by name and location as follows:

B\&O Cuntis Bay Coal Piers, Baltımore, Marytand

B8O Curtis Bay Coal Piers. Baltimore, Maryland
C\&O Coal Piars Nos. 14 \& 15 . Newpon News, Virginia

N\&W Lamberts Point Coal Piers Nos. $5 \& 6$, Norfolk, Virginia
AlW

Alabama State Docks Bulk Handling Plant, Mobile, Alabama

Alabama State Docks McOuthie Terminals. Mobilo, Alabama

Canton Coal Pier, Batimore Harbor on Chesapeake Bay

Greenwich Coal Pier, Groertwich Point, Philadelphia, PA, on Delaware River

Port Richmond Pier, Pier 18 - Pon Richmond, Philadelphia, PA, on Delaware River

Galveston Regional Coal Distribution Center. Pelican Istand, Galveston, Texas

International Manne Terminals.Plaquemines Parish Terminal, Mile 57 AHP. Mississipp River, Aporox, 30 miles South of New Orleans

Energy Terminals of Houston, Inc. a Subsidiary of Soros Associates. Houston. Texas

The Coastal Ports include those located at Charleston, SC. New York, NY. San Diego. CA, Los Angeles, CA, and Seattie. WA. 
5. Truck: Shipments of coal moved to consumers by truck. Not included is coal hauled to or away from a rallroad siding by truck

6. Tramway/Conveyor or Siury Pipeline: Shipments of coal moved to consumers by tramway, conveyor, or slurry pipeline.

C. Other Uses

1. Rallroad Fuel: Shipments of coal to railroaci companies to be used as fuel (engine. powerhouse, elc.).

2. Veasellship Ful: Shipments of coal to be used as vesselship fuel

3. Coel Uead at Your Compeny' Minew: Coal consumed at your company's mines in this State during the reporting quarter

4. Coal Sold to Employeces: Coal sold to employees during the reporting quarter.

\section{Miscellanoous}

1. Parent Company: A company which solely or jointly owns the reponing company and which is not itsell a subsidiany of or owned by another company

\section{Vil. General instructions}

A separate report shall be submitted for each coal-producing State in which coal was mined. Complete all data items on the form applicable to your operation. All ligures shoul be rounded to the nearest whote number. Report all tonnage in short tons (2.000 pounds). Blanks will be interpreled as zeroes; therelore it is not necessary to enter "not applicable be rounded to the nearest whole number. Report all tonnage
(NA)" or dashes or zeroes where you have no data to repon.

\section{Specific Instructions}

How to report the methods of transporting the coal to a consumer:

A. If the shipment imvolved only one method of transportation from the point of origin to the consumer, report the shipment in the section for that method of transportation.

B. If the shipment involved more than one method of transportation from the point of origin to the consumer, use the lollowing guidelines in reporting the method of transportation

1. H water transportation was involved, regardess of other methods of transportation also involved, report the method of transportation as one of the three types of water shlpments, i.e., River, Great Lakes, or Thdemwater Piers and Coestal Ports. In addition, specity how the shipment(s) was (were) delivered to the river (rail, truck. or tramway' conveyor). Great Lakes (rall, truck, or river), or Tidewater Piers and Coastal Ports (rail, truck, or river).

2. It rall and truck are involved and no water transportation is involved, then repon the method of transportation as rail.

3. Truck is to be reported as the method of transportation to the consumer only it the coal was shipped directly to the consumer by iruck.

\section{Disclosure of Information}

The name and address of the responding company, and location (coal-producing state) will be released upon request in the form of a full or partial list of respondents for this coal data collection survey.

The information contained on this form will be kept confidential to the extent that it satisfies the criteria for exemption in the Freectom of Information Act (FOIA). the DOE regulations implementing the FOIA, and the Trade Secrets Act, 18 U.S.C. \$1905.

Upon receipl of a request for this information under the FOIA, the DOE shall, in accordance with the procedures and criteria provided in $10 \mathrm{C}$.F.R. \$1004.11, make a final determination whether the information is rxempt from disckesure. To assist in this determination, respondents should demonstrate tu the DOE that, for example, their information contains trade secrets or commercial or financial information whose release would be likely to cause substantial harm to their company's competitive position. A letter accompanying the submission that explains (on an elersem-by-elemem basis) the reasons why the information would be likely lo cause the respondent subetantial competitive harm if released to the public would aid in this determination. A new justification does not need to be provided each time intormation is subunitred on the form, if the company has previously submitted a justification for that information and the justification has not changed.

Except as othenwise provided by lew. the intormation may be made available in response to an order of a Coun of competent furisdiction, or. upon request, to other components of the Department of Energy, to any Committes of Congress, the General Accounting Office, or other crongressional agencies authorized by law lo receive such information. Delailed provisions of the restrictions on the disclosure of this information can be lound in the Policy on the Disclosure of Individually ldentifiable Energy information in the Possession of the Energy Information Administration (45 Federal Register 859812 (1980))

\section{Originating States:}

\begin{tabular}{ll} 
State & State \\
\hline Alabama & Montana \\
Alaska & New Mexico \\
Arizona & North Dakota \\
Arkansas & Otio \\
Calitomia & Oklahoma \\
Colorado & Pennsylvania anthracite \\
Illinois & Pennsytvania bituminous \\
Indiana & Tennessee \\
lowa & Texas \\
Kansas & Utah \\
Kentucky Eastem & Virginia \\
Kentucky Westem & Washington \\
Louisiana & West Virginia Non: :orn \\
Maryland & West Virginia Southem \\
Missouri & Wyoming
\end{tabular}

Kentucky, Eastern - All mines not in the counties of Western Kentucky as defined below:

Kentucky, Western - All mines in the following counties in Wesiem Kentucky: Butler, Caldwell, Christian. Crittenden, Daviess, Edmonson, Grayson, Hancock. Henderson, Hopkins, Logan, McLean, Muhlenberg, Ohio. Simpson, Tood, Union, Warren, and Webster.

Pennaylvanie anthracite - All mines in the following counties: Carbon. Columbia, Dauphin, Lackawanna, Lebanon, Luzerne, Northumberland, Schuylkill. Sullivan, and Susquehanna. All anthracite mines in Bradtord County.

Pennayivania bliuminous - All mines not in the counties of Pennsylvania anthracte as defined above and all bituminous mines in Bradtord County.

Weat Virginla, Northern - All mines in the following counties (formerty defined as Coal-Producing Districts 1, 3, 6): Barbour, Brooke, Braxton, Calhoun, Doddridge, Gilmer, Grant, Hancock, Harrison, Jackson, Lewis, Marion, Marshall, Mineral, Monongalia, Otio, Pleasants, Preston, Randolph, Ritchie, Roane, Taylor, Tucker, Tyler, Upshur, Webster, Wetzel, Wirt, and Wood.

Weat Vlrginla, Southern - All mines in the following counties (formerly defined as Coal-Producino Districts 7 \& 8): Boone, Cabell, Clay, Fayette, Greenbrier, Kanawha, Lincoln, Logan, Mason, McDowell, Mercer, Mingo, Monroe, Nicholas, Pocahontes, Putnam, Raleigh, Summers, Wayne, and Wyoming. 


\section{Glossary}

Anthracite: Anthracite, or hard coal, is the highest rank of economically usable coal. It is jet black with a high luster. The moisture content generally is less than 15 percent. Anthracite contains approximately 22 to 28 million Btu per ton as received and averages about 25 million Btu per ton. Its ignition temperature is approximately 925 to 970 degrees Fahrenheit. Virtually all of the anthracite mined is from northeastern Pennsylvania. It is used mostly for space heating and generating electricity.

Bituminous Coal: Bituminous coal, or soft coal, is the most common coal. It is dense, black, often with welldefined bands of bright and dull material. Its moisture content usually is less than 20 percent. The heating value ranges from $1^{4}$ to 30 million Btu per ton as received and averages about 24 milition Btu per ton. The ignition temperature ianges from about 700 to almost 900 degrees Fahrenheit. Bituminous coal is mined chiefly in the Appalachian and Interior coal fields. It is used for generating electricity, making coke, space heating, and supplying heat for industrial use.

Census Divisions: The nine geographic divisions of the United States established by the Bureau of the Census, U.S. Department of Commerce for statistical analysis. The boundaries of Census divisions coincide with State boundaries. In some cases, the Pacific Division is subdivided into the Pacific Contiguous and Pacific Noncontiguous areas.

Coal-Producing States: The States where mined and/ or purchased coal originates. defined as follows: Alabama, Alaska, Arizona, Arkansas, California, Colorado, Illinois. Indiana, Iowa, Kansas. Kentucky Eastern, Kentucky Western, Louisiana, Maryland, Missouri, Montana, New Mexico. North Dakota, Ohio, Oklahoma, Pennsylvania anthracite. Pennsylvania bituminous. Tennessee, Texas, Utah, Virginia, Washington, West Virginia Northern. West Virginia Southern, and Wyoming.
The following Coal-Producing States are split in origin of coal, as defined below:

Kentucky, Eastern: All mines not in the counties of Western Kentucky as defined below:

Kentucky, Western: All mines in the following counties in Western Kentucky: Butler, Caldwell, Christian, Crittenden, Daviess, Edmonson, Grayson, Hancock, Henderson, Hopkins, Logan, McLean, Muhlenberg, Ohio. Simpson. Todd, Union, Warren, and Webster.

Pennsylvania anthracite: All mines in the following counties: Carbon, Columbia, Dauphin, Lackawanna, Lebanon, Luzerne, Northumberland, Schuylkill, Sullivan, and Susquehanna. All anthracite mines in Bradford County.

Pennsylvania bituminous: All mines not in the counties of Pennsylvania anthracite as defined above and all bituminous mines in Bradford County.

West Virginia, Northern: All mines in the following counties (formerly defined as Coal-Producing Districts 1. 3, \& 6): Barbour, Brooke, Braxton, Calhoun, Doddridge, Gilmer, Grant, Hancock, Harrison, Jackson, Lewis, Marion, Marshall. Mineral, Monongalia, Ohio, Pleasants, Preston, Randolph. Ritchie, Roane, Taylor. Tucker, Upshur, Webster, Wetzel, Wirt, and wood.

West Virginia, Southern: All mines in the following counties (formerly defined as Coal-Producing Districts 7 \& 8): Boone, Cabell, Clay, Fayette, Greenbrier, Kanawha, Lincoln, Logan, Mason, McDowell. Mercer, Mingo, Monroe, Nicholas, Pocahontas, Putnam, Raleigh, Summers. Wayne, and Wyoming.

Coal-Producing Regions: A geographic classification of coal-producing States. The States in the Appala- 


\section{Your Entrée to Federal Statistics!}

\section{CENSUS CATALOG AND GUIDE: 1991}

\section{Helps you select from all the proclucts}

Every Census Bureau product issued mid-1988-90:

Reponts Microfiche Computer tapes CD-ROM

Maps Floppy disks Online access

Key statistical publications from other Federal agencies

\section{Features facts about each product}

Topics Areas Dates Prices

\section{Makes finding the right product easy}

Extensive overview chapter Title index Series index

Detailed subject index Guides to each statistical subject

Identifies sources of assistance

1,400 State Data Center organizations

200 Census Bureau specialists

1,500 depository libraries

Other Federal statistical agencies

5 other directory lists

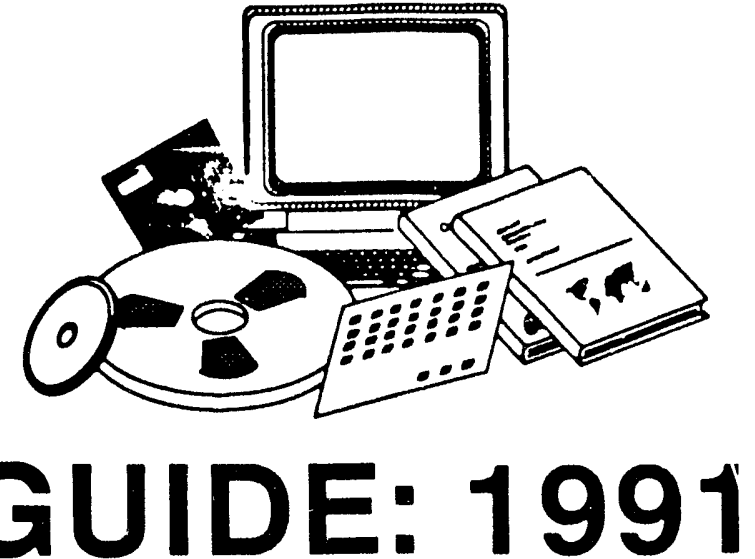

\section{Contents}

General and Reference

Agriculture

Business

Construction and Housing

Foreign Trade

Geography

Governments

International

Manufacturing and Mineral Industries

Population

Transportation

1990 Census Products (NEWI)

Sources of Assistance

Factinder Guides

\section{*6059}

Yes, please send me: Superintendent of Documents Publication Order Form coples of Census Catalog and Gulde: 1991 at \$15, S/N 003-024-07271-8

1. The total cost of my order is $\$$ (International customers please add $25 \%$.) All prices include regular domestic postage and handling and are good through 1/92. After this date, please call Order and Information Desk at 202-783-3238 to verity prices.

Charge your order It's easy!

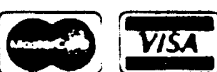

2. Please Type or Print

(Company or personal name)

(Additional address/attention line)

(Stroet address)

(City, State, ZIP Code)

(Daytime phone including area code)
3. Please Choose Method of Payment:

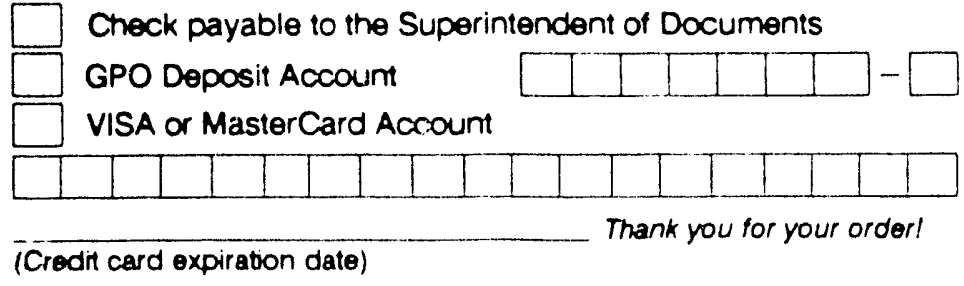

(Signature)

4. Mall To: Superintendent of Documents, Government Printing Office, Washington, DC 20402-9325 (Charged orders may be sent by FAX. The number is 202-275-0019.) 

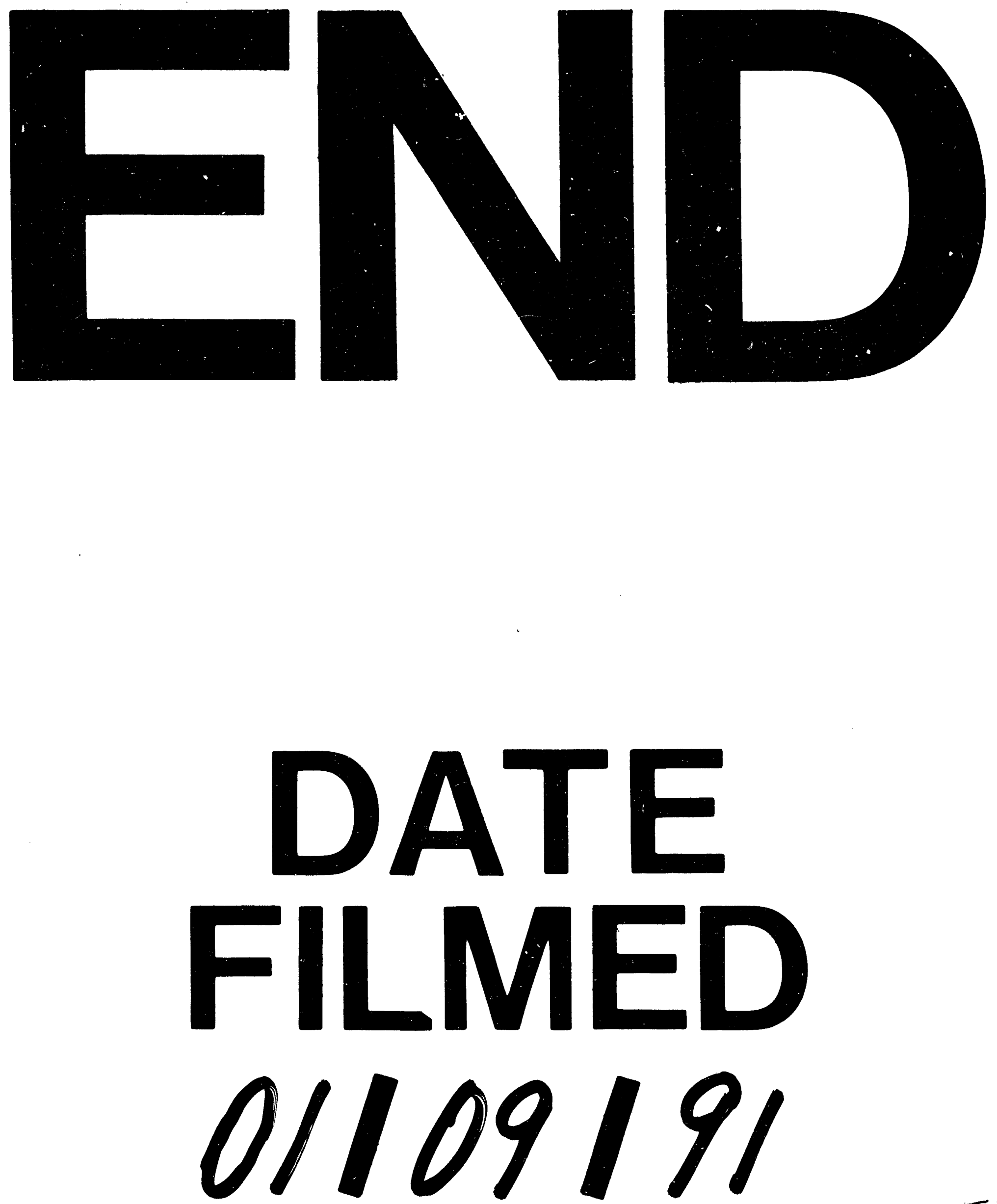

$$
-\because
$$

